\title{
Structural and Magnetic Properties of \\ Cobalt Implanted Diamond-like Carbon Films \\ Synthesized by Ion Beam Methods
}

BY

PRASANTH GUPTA SRIDHAR GUPTA

A thesis submitted to the Victoria University of Wellington in fulfilment of the requirements for the degree of Doctor of Philosophy

Victoria University of Wellington

2017 



\section{Abstract}

Novel methods to synthesize and modify the structure, composition, magnetic behavior and electrical conductivity of thin films are of tremendous importance for fabrication of nano-devices. This research focuses on the application of ion beam methods to produce diamond-like carbon thin films and incorporate magnetic nanostructures within them for potential spintronic applications. Diamond-like carbon films were synthesized by ion beam deposition and were implanted with magnetic ions at low energy to induce magnetic order in near-surface region of the thin film. This research work focuses on the exotic ion-solid interactions that occur between the energetic ions and the base matrix and their subsequent effects on the structure, atomic distribution and magnetic behavior of the implanted films. The results are of significant interest to both, ion beam community and spintronics industry.

Diamond-like carbon (DLC) is an amorphous carbon material that contains significant fraction of its carbon bonded in $\mathrm{sp}^{3}$ hybridization. DLC films were produced by high energy ion beam deposition, specifically by both, direct ion beam deposition and mass selective ion beam deposition. Raman spectroscopy revealed that DLC films deposited by molecular ion beams $\left(\mathrm{C}_{3} \mathrm{H}_{6}^{+}\right)$with $5 \mathrm{keV}$ deposition energy has 30 - $50 \%$ of its carbon bonded in $\mathrm{sp}^{3}$ hybridization. The $\mathrm{sp}^{3}$ content was observed to decrease with increase in deposition energy. The hydrogen content in the deposited films was measured to be $25-30$ at.\% by resonant nuclear reaction analysis. The selection of deposition energy and ion species was observed to have strong influence on the thin film properties.

Cobalt was chosen as the preferred magnetic ion for doping diamond-like carbon thin films. Co was implanted at low energy $(30 \mathrm{keV})$ and at room temperature. The implantation fluence (incident ions per unit area) was varied from $0.8-20 \times 10^{16}$ atoms.cm $^{-2}$. The implantation current density was limited to $5 \mu \mathrm{A} . \mathrm{cm}^{-2}$ to prevent any bulk heating effects. Monte-Carlo simulations suggest the implantation profile to be single Gaussian with a projected range of $\sim 37 \mathrm{~nm}$. High resolution Rutherford backscattering spectrometry (HR-RBS) however showed that the Co distribution varied from a unimodal distribution at low fluences $\left(<1.5 \times 10^{16}\right.$ atoms.cm $\left.{ }^{-2}\right)$ to an asymmetric bimodal distribution at high fluence $\left(7 \times 10^{16}\right.$ atoms.cm $\left.{ }^{-2}\right)$. Furthermore, a steady state condition was reached at an implantation fluence of $\left(12 \times 10^{16}\right.$ atoms.cm $\left.{ }^{-2}\right)$ which is not expected from the simulations. Cross-sectional TEM imaging and corresponding fast Fourier transform analysis reveals that the implanted Co atoms precipitate into cobalt carbide nanoparticles. At high fluences, the nanoparticles accumulate at two regions : (i) in the immediate near-surface region (nanoparticles as large as $5 \mathrm{~nm}$ ) (ii) at the projected range of implanted ions (large density of $\sim 2$ $\mathrm{nm}$ nanoparticles) in agreement with the measured Co distribution.

The bimodal distribution along with the nanoparticle formation can only be explained 
by the occurrence of non-ballistic processes such as precipitation and localized diffusion during Co implantation. These processes are enhanced by energy deposited during collision cascades, relaxation of thermal spikes, and defects formed during ion implantation. A key factor responsible for the manifestation of these effects, is the presence of hydrogen in the base matrix.

Cobalt implantation was carried out on amorphous carbon films (lacking hydrogen) at the same implantation conditions. HR-RBS and TEM measurements on these films show that Co assumes a unimodal distribution when hydrogen is absent in the base matrix. Resonant nuclear reaction analysis shows that ion implantation leads to massive redistribution of hydrogen atoms in the DLC films. Raman spectroscopy further indicate that ion implantation causes graphitization of DLC films. These results show that the energetic ion interactions in DLC matrix are unique and lead to interesting elemental distribution and structural effects.

Cobalt containing nanoparticles in the DLC films lead to room-temperature magnetic order. Magnetic measurements were carried out using a magnetic property measurement system employing a superconducting quantum interference device. The temperature dependence of saturated moment observed from the magnetic measurements of low fluence samples resembles the behavior of a spin glass material. This is explained by the structural disorder present in the magnetic nanoparticles. Increasing the implantation fluence, reduces the spin glass contribution and leads to formation of ordered magnetic phases within the nanoparticles. This is confirmed by both the TEM data and observation of ferromagnetic contribution in addition to spin glass behavior in the temperature dependent magnetization measurements of high fluence sample. Low field magnetic measurements further reveal that the nanoparticles exhibit superparamagnetic behavior. Interestingly, the measurements also indicate presence of dipole-dipole interaction between the nanoparticles.

As deposited DLC films were observed to be electrically insulating. Co implantation resulted in the formation of metallic nanoparticles which is measured to have increased the conductivity of DLC by nearly six order of magnitude. Measurement of electrical conductivity as a function of temperature indicates the dominant conduction mechanism to arise from the tunneling between the large disordered metallic nanoparticles found in the near surface region. Since the particles are randomly oriented, the electrons can tunnel through different pathways and the overall behavior is observed to have a power law dependence over the measurement temperature. At higher temperature, an additional contribution is observed to arise from the smaller nanoparticles positioned deeper inside the implanted DLC films. This contribution can be semiconducting or metallic in nature and is dependent on the implanted Co concentration. The overall conduction behavior of Cobalt implanted DLC is modeled as a network of two parallel resistors with each resistor arm, representing the contribution from 
the larger and smaller nanoparticles respectively.

This research overall shows that ion implantation into DLC can harness the effects of collision cascades, thermal spikes and defect formation for producing magnetic nanoparticles with unique elemental distributions. The implanted DLC films exhibit novel magnetic and transport properties which can ultimately lead to the development of spintronic devices.

Key results from this research are published in physics journals such as Applied Physics Letters, Journal of Physics: Applied Physics D, Nuclear Instruments and Methods:B which are the relevant journals for this research work. 



\section{Acknowledgment}

This work would not have been possible without the help and support from a large number of people. My first and foremost thanks is to my supervisors Dr Andreas Markwitz and Dr Grant Williams. Most of my learning about ion beam physics stems directly from the many hours of discussion I had with Dr Andreas Markwitz. I am grateful for his constant support throughout the $\mathrm{PhD}$, both professionally and personally. My sincere gratitude goes to Dr Grant Williams for his valued assistance and patience. His strong knowledge in solid state physics and attention to detail has helped me produce quality research work.

I am grateful to all the collaborators for their contribution in this research work. I am thankful to Dr Thomas Osipowicz and Dr Saumitra Vajandar for hosting me at the Center for Ion Beam Applications, Department of Physics, National University of Singapore, Singapore. Their support with high resolution Rutherford backscattering spectrometry measurements were crucial for this research. Dr Hans-Werner Becker and Dr Detlef Rogella from RUBION, Ruhr University, Bochum, Germany has carried out resonant nuclear reaction analysis on many of my samples. I am grateful for their valuable contribution. I am also thankful to Dr Rene Huebner from Institute of Ion Beam Physics and Materials Research, HelmholtzZentrum, Dresden- Rossendorf, Germany for carrying out the TEM measurements. My sincere thanks to Dr Shen Chong for teaching me usage of SQUID and PPMS at Robinson Research Institute, New Zealand. I also acknowledge the contribution from Berit Mohr and Konrad Suschke for their work on DLC at GNS Science.

I am grateful to the entire staff of Materials and Air department at GNS Science for their kind words and encouragement throughout my PhD. I am deeply indebted to Dr John Kennedy for his constant support and guidance. I am immensely grateful to Dr Jerome Leveneur for the several thought provoking conversations we had. These discussions urged me to critically examine my results. Many thanks to Dr Peter Murmu for his kind words and encouragement. I thank Dr Tushara Prakash and Dr Pierre Couture for their support and friendship. I would like to thank Chris Purcell and John Futter for their technical support which enabled me to produce successful results. I had the pleasure to work with intern students Morgane Rondeau, Ryan Yang and Sascha Kubitzky during my PhD. I am grateful for their contribution.

I acknowledge the funding from The MacDiarmid Institute for Advanced Materials and Nanotechnology, New Zealand and Victoria University of Wellington for my PhD. I am very grateful for their support.

Finally, I thank my family and my friends for their love and affection. My profound gratitude goes to my uncle Sankar Gupta and my mom Dhanalakshmi. Their constant support and motivation were vital for the successful completion of this thesis. I thank my 
grandparents for their blessings and I dedicate this thesis to my brothers. My final thanks goes to my wife and my best friend, Sowmiya Murali. Her support and motivation made this writing process an enjoyable experience.

Thank you. 




\section{Contents}

1 Introduction $\quad 1$

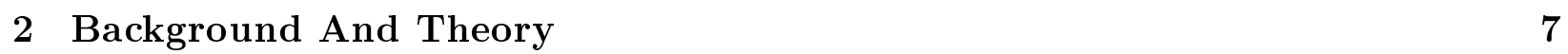

2.1 Ion-solid Interactions . . . . . . . . . . . . . . . . 7

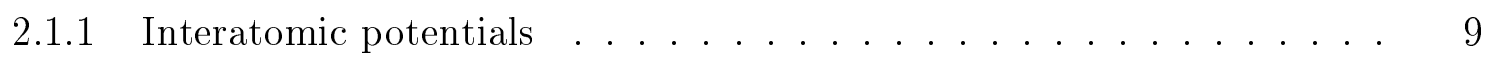

2.1.2 Dynamics of binary elastic collisions . . . . . . . . . . 12

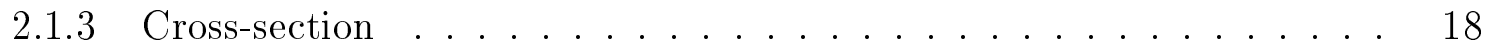

2.1 .4 Ion Stopping . . . . . . . . . . . . . . . 21

2.1.4.1 Nuclear stopping . . . . . . . . . . . . 22

2.1.4.2 Electronic stopping . . . . . . . . . . . 23

2.1.5 Ion Range and Straggling . . . . . . . . . . . . . . 28

2.1.6 Atomic displacements and Sputtering . . . . . . . . . . . . . . 32

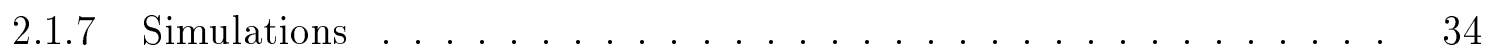

2.2 Magnetic Properties . . . . . . . . . . . . . . . 35

2.2.1 Origin of magnetism . . . . . . . . . . . . . . 35

2.2.2 Classification of magnetic materials . . . . . . . . . . . 36

2.2.3 Temperature and field dependence . . . . . . . . . . . 37

2.2.4 Magnetic nanoparticles . . . . . . . . . . . . . 39

2.3 Conclusion . . . . . . . . . . . . . . . . . . 40

3 Methods $\quad 41$

3.1 Ion implantation . . . . . . . . . . . . . . . . . . 42

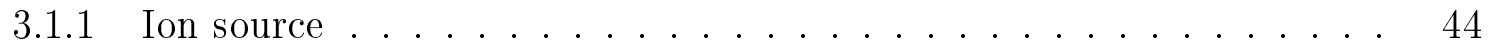

3.1.2 Ion extraction . . . . . . . . . . . . . . . 45

3.1 .3 Ion beam focusing . . . . . . . . . . . . . 47

3.1 .4 Mass separation . . . . . . . . . . . . . . 48

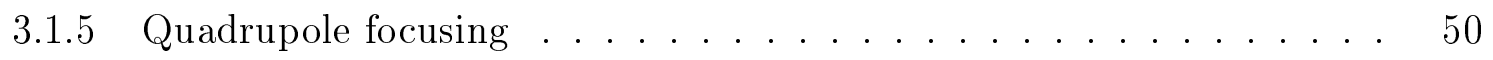

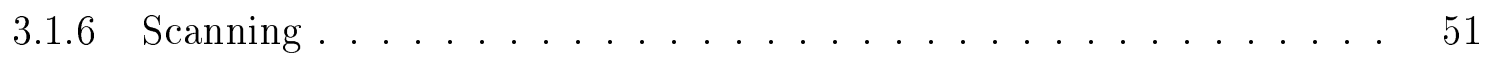


3.1 .7 Sample Chamber . . . . . . . . . . . . . . . . . . . . . . 51

3.2 Direct Ion Beam Deposition . . . . . . . . . . . . . . . . . 52

3.3 Mass-Selective Ion Beam Deposition . . . . . . . . . . . . . . . . . 54

3.4 High resolution Rutherford backscattering . . . . . . . . . . . 56

3.4 .1 Data Stitching. . . . . . . . . . . . . . . . . . 61

3.4 .2 Calibration . . . . . . . . . . . . . . . . . 62

3.4 .3 SIMNRA fitting . . . . . . . . . . . . . . . 63

3.5 Cross-sectional Transmission Electron Microscopy . . . . . . . . . . . 63

3.6 Resonant Nuclear Reaction Analysis _ . . . . . . . . . . . . . . 67

3.7 Raman Spectroscopy . . . . . . . . . . . . . . . . . . . . 68

3.8 Magnetic property measurement system $\ldots \ldots \ldots \ldots \ldots$

3.9 Four terminal transport measurement . . . . . . . . . . . . . . 73

4 Ion beam deposition of diamond like carbon $\quad 75$

4.1 Introduction . . . . . . . . . . . . . . . . . 75

4.2 Diamond-like carbon . . . . . . . . . . . . . . . 75

4.3 Growth mechanism . . . . . . . . . . . . . . . 77

4.4 Mass-selective Ion beam deposition . . . . . . . . . . . . . . 80

4.5 Direct Ion beam deposition . . . . . . . . . . . . . . . . . . . 90

4.6 Effect of Ion Energy . . . . . . . . . . . . . . . . . . . . 92

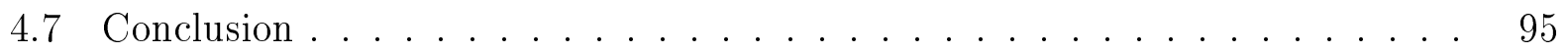

$\begin{array}{llr}5 & \text { Structure and Distribution } & \mathbf{9 7}\end{array}$

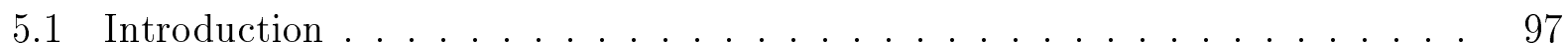

5.2 Ion Implantation $\ldots \ldots \ldots$

5.3 Simulations . . . . . . . . . . . . . . . . . . . . 98

5.3 .1 Ion fluence . . . . . . . . . . . . . . . . . . . . . . . 101

$5.3 .2 \quad$ Sputtering . . . . . . . . . . . . . . . . . . . . . . 103

5.4 Co distribution in implanted DLC . . . . . . . . . . . . . . 106

5.4.1 Low fluence - Unimodal distribution . . . . . . . . . . . . . . 114

5.4 .2 Moderate fluence I : Emergence of bimodality . . . . . . . . . . 118

5.4 .3 Moderate fluence II: Asymmetric bimodal distribution . . . . . . . 123

5.4 .4 High fluence: Enhanced bimodal distribution . . . . . . . . . . . . 126

5.4 .5 Saturation: Steady state condition _ . . . . . . . . . . . 131

5.4 .6 Possible causes for bimodality . . . . . . . . . . . . . . . . 134

5.5 Nanoparticle Formation $\ldots \ldots \ldots 136$

5.5.1 Elemental composition from STEM . . . . . . . . . . . . 139 
5.5.2 Nanoparticle size distribution . . . . . . . . . . . . . . . 146

5.6 Model - Evolution of bimodality . . . . . . . . . . . . . . . . . 149

5.7 Conclusion . . . . . . . . . . . . . . . . . . . . . 154

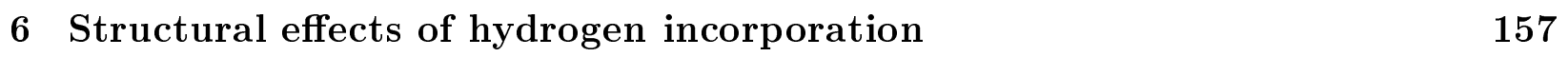

6.1 Introduction . . . . . . . . . . . . . . . . . . . 157

6.2 Effect of hydrogen on Co profile . . . . . . . . . . . . . 158

6.2 .1 DTRIM Simulation . . . . . . . . . . . . . . . 158

6.2 .2 Cobalt distribution . . . . . . . . . . . . . . . . . . . 159

6.2 .3 Cross sectional imaging . . . . . . . . . . . . . . . . . . 163

6.3 Nuclear Reactions - Hydrogen measurement . . . . . . . . . . . . . 165

6.4 Raman Spectroscopy . . . . . . . . . . . . . . . . . . 178

6.5 Conclusion . . . . . . . . . . . . . . . . 183

7 Magnetic and Transport Properties 185

7.1 Introduction . . . . . . . . . . . . . . . . . . 185

7.2 Magnetic Properties . . . . . . . . . . . . . . . . . 185

7.3 Electrical conductivity . . . . . . . . . . . . . . 200

7.4 Conclusion . . . . . . . . . . . . . . . . . . 211

8 Summary and Outlook $\quad 213$

8.1 Summary . . . . . . . . . . . . . . . . . . . . . . 213

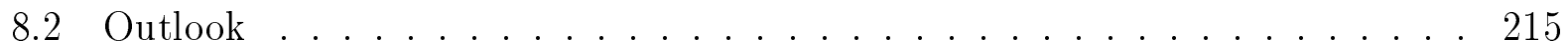




\section{List of Figures}

2.1 Schematic of ion interactions with matter. An energetic ion bombarding on a material surface reaches an average distance $R_{p}$ by loss of energy due to atomic collisions and electronics excitations. Redrawn from [1] . . . . . . .

2.2 Schematic of Interatomic forces and interatomic potential as a function of interatomic distance. As an atom moves away from its equilibrium position it experiences a repulsive or an attractive force to reinstate its equilibrium position and its strength depends on the interatomic potential between its neighboring atoms. Redrawn from $[1] \ldots \ldots \ldots$

2.3 Schematic representing binary elastic collision in laboratory frame of reference. An projectile atom of mass $M_{1}$ with initial energy $E_{0}$ and initial velocity $v_{0}$ collides with a stationary atom of mass $M_{1}$ resulting in transfer of energy and momentum. Immediately after collision the projectile atom and the recoiled atom are noted to move in different directions with energy $E_{1}$ and $E_{2}$ and velocity $v_{1}$ and $v_{2}$ respectively. Redrawn from $[1] \ldots \ldots \ldots$

2.4 Schematic representation of binary elastic collision in laboratory frame of reference and center-of-mass frame of reference. Redrawn from [1] . . . . . .

2.5 Schematic representing collision trajectory and impact parameter. The impact parameter $b$ as represented in the figure is the perpendicular offset between the collision trajectory of the projectile atom and a parallel line passing through the center of mass of the target atom. It can be seen that the impact parameter and minimum distance of separation during collisional process are inter-related. Redrawn from $[1] \ldots \ldots \ldots \ldots$

2.6 Schematic representing the effective cross-section for ion-atom collision in a thin foil. An energetic ion beam impringing a thin foil of unit area and traversing a thickness $d x$ experiences collisions whose number is proportional to the atomic density of the thin foil, the thickness traversed by the ion in the process and the cross-section of the target atoms for collision. Redrawn from [1] 18 
2.7 Schematic representing dependence of scattering angle on impact parameter. For a projectile atom to be scattered within a specific window of scattering angle it needs to be colliding with the target atom within a specific window of impact parameter. As the impact parameter increases the scattering angle decreases. Redrawn from $[1] \ldots \ldots$. . . . . . . . . . . .

2.8 Schematic representing a typical ion beam analysis setup where the a detector is placed at a specific position and angle to detect the signal from the ion-atom interaction. The signal can the scattered incident ion beam, the recoiled target atoms or ionizing/electromagnetic radiation released from the collisional process. RBS assumes a similar setup where the detector detects the scattered incident beam at a particular scattering angle and a thus specific solid angle.

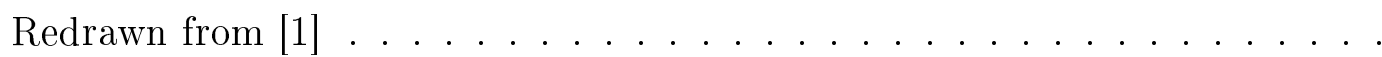

2.9 Schematic representing the dependence of differential cross-section of solid angle on impact parameter. Similar to scattering angle for ion beam scattering to result in a scattered beam within a window of solid angle, the incident beam must lie within a window of impact parameter. Redrawn from [1] . . . . .

2.10 Schematic representing quasi particle formed during ion-atom collision with impact parameter $b$ and distance of separation $r$ as proposed by Frisov. Projectile atom with atomic number $Z_{1}$ and velocity $v$ forms the P-region of the quasiparticle and the target atom with atomic number $Z_{2}$ forms the T-region of the quasiparticles. Frisov defined a plane bisecting the line joining the center of the two particles. Electrons passing through this Frisov plane strongly interact with the force field of the corresponding atoms and results in energy loss by electron exchange. Redrawn from [1] . . . . . . . . . . . . . .

2.11 Plot of nuclear and electronic stopping with respect to $\log (\mathrm{E})$. It can be observed that the nuclear stopping dominates the energy loss of the projectile at low energy region and the electronic stopping dominates at high energy region. This implies that during ion implantation and ion beam deposition the stopping cross-section that is of importance will be nuclear stopping and in ion beam analysis such as RNRA and RBS electronic stopping will be the key cross-section to be considered [1] . . . . . . . . . . . . .

2.12 Schematic representing the different range parameters of an ion implanted into the target surface. Redrawn from [1] . . . . . . . . . . . . 
2.13 Schematic representing electron contribution to magnetic moment. Two contributions as observed in the figure are the magnetic moment due to orbital angular momentum of the electron and magnetic moment arising from the intrinsic angular momentum of the electron. Redrawn from [2] . . . . . . .

2.14 Magnetization and magnetic susceptibility of different magnetic materials with varying field and temperature. Redrawn from [2] . . . . . . . . . . 38

3.1 Industrial implanter at GNS Science . . . . . . . . . . . . . 43

3.2 Schematic representing Penning Ion source . . . . . . . . . . . . . 45

3.3 Schematic representing ion extraction from ion source to the injector system starting with the Einzellens. The separation distance between them has to increased with increasing terminal voltage to prevent arcing and improve the transmission efficiency of the ion beam generated from the ion source. . . . . 46

3.4 Schematic representing 2D cross-section of ion path in the Einzellens. The ions entering the Einzellens are usually focused to the center of the double focusing electromagnet used for mass-separation in the ion implanter. . . . . 47

3.5 Schematic representing ion focusing and steering in the ion implanter . . . . 48

3.6 Schematic representing mass separation in Industrial Implanter at GNS Science 49

3.7 Duplet quadrupole system at the Industrial Ion implanter . . . . . . . . . . . 51

3.8 Direct ion beam deposition system at GNS Science. Source [3] . . . . . . . 53

3.9 Schematic representing mass selective ion beam deposition system at GNS

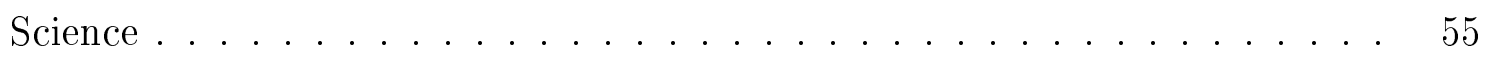

3.10 Layout of the accelerator and beamlines at CIBA, NUS [4] . . . . . . . 57

3.11 HR-RBS detector system. Source [5] . . . . . . . . . . . . . . 59

3.12 Raw data of sample Co implanted DLC obtained from HR-RBS measurement 61

3.13 Stitched raw HR-RBS data of Co implanted DLC film . . . . . . . . . . . 62

3.14 TEM lamella of Co implanted DLC film prepared by FIB milling . . . . . 65

3.15 Schematic representing transitions in electronic energy levels. . . . . . . . 66

3.16 Schematic representing excitation of electrons on incidence of electromagnetic radiation. Most electronic excitations result in release of the incident photon without change in energy level. A minor fraction of the electrons after excitation do not relax back to their initial energy level and the difference of energy between the initial energy level and the final relaxed energy level is observed in the photon emitted during relaxation. These transitions are referred to as Raman transitions and probing these transitions provides information about the different phonon states of the target material. . . . . . . . . . . 
3.17 Schematic of superconducting quantum interference device composed of a closed superconducting loop with two Josephson junction (X and W). Source

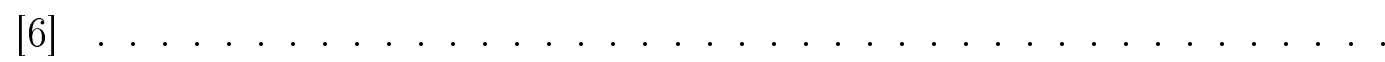

3.18 Schematic of a SQUID gradiometer used to detect the magnetic moment of the sample. The gradiometer design and operation allows capturing the induced magnetic moment in the sample as an induced voltage in the pick-up coils without interference from the surrounding magnetic fields. Source [7] . . . 72

3.19 Co implanted DLC films on Si substrate loaded for four terminal transport measurement. . . . . . . . . . . . . . . . . 74

4.1 Schematic representing the $\mathrm{sp}^{3}, \mathrm{sp}^{2}$, sp hybridized bonding. Redrawn from [8] 76

4.2 Ternary phase diagram of bonding in amorphous carbon-hydrogen alloys. The figure classifies different types of DLC based on their hydrogen configuration and $\mathrm{sp}^{2}: \mathrm{sp}^{3}$ ratio. Source $[8] \ldots \ldots \ldots$. . . . . . . . . . 76

4.3 Berman-Simon phase diagram for carbon representing the conditions required for synthesis of diamond. Source [9] . . . . . . . . . . . . 78

4.4 Schematic diagram of densification by subplantation. An incident carbon / carbon-rich beam upon implantation introduces carbon (implanted) interstitials at end of its range causing layer densification which is favorable for formation of $\mathrm{sp}^{3}$ hybridization. Those carbon atoms that relax back to surface do not experience the pressure available at the densified layer and hence grows an $\mathrm{sp}^{2}$ rich surface. Redrawn from $[8] \ldots \ldots \ldots \ldots$

4.5 Schematic representing subplantation of single and molecular ion. It can be seen that use of molecular ion expands the cascade region caused during implantation. Redrawn from $[8]$. . . . . . . . . . . . . 79

4.6 Schematic representing difference in $\mathrm{C}$ and $\mathrm{H}$ penetration range into Si substrate 81

4.7 Schematic representing growth of DLC by MSIBD. During the initial stage of growth, the carbon atoms are concentrated near the projected range. As the deposition continues the concentration of the carbon atoms in the Si substrate increases further to the point of saturation. As the process continues further increase in carbon concentration results in outgrowth of carbon atoms onto the surface resulting in formation of a carbon layer. . . . . . . . . . . 82

4.8 Cross-sectional TEM images of MSIBD deposited DLC at different length scales 84

4.9 High Resolution RBS spectrum of as-deposited DLC . . . . . . . . . 85 
4.10 Hydrogen profile in as-deposited DLC films synthesized by MSIBD. The raw data is corrected for hydrogen loss during measurement and is overlaid on top of it. It can be seen from the corrected data that the significant decline in hydrogen concentration as function of depth arises primarily from the experimental artifact. The hydrogen concentration within the DLC films was observed to within $25-30$ at.\%. . . . . . . . . . . . .

4.11 Raman spectrum of as-deposited DLC films synthesized by MSIBD. The data is fit with two Gaussian peaks representing the D (green) and G (red) peak whose sum fits the measured data well. . . . . . . . . . . . . . . . 89

4.12 Cross-sectional TEM image of as-deposited DLC synthesized by DIBD . . 92

4.13 Raman analysis of DIBD and MSIBD deposited DLC as a function of acceleration voltage. In the case of DIBD films it can be seen that the $\mathrm{I}(\mathrm{D}) / \mathrm{I}(\mathrm{G})$ ratio and $G$ peak position shows significant increase with acceleration voltage, whereas for MSIBD film the changes are not significant. This indicates that for DIBD, increase in acceleration voltage results in increase in overall $\mathrm{sp}^{2}$ concentration. While for MSIBD, increase in acceleration voltage increases the size of $\mathrm{sp}^{2}$ clusters. . . . . . . . . . . . . . . .

4.14 Three stage model proposed by Ferrari and Robertson describing the transition of carbon from crystalline graphite to tetrahedral amorphous carbon. The model plots the trend taken by two measured parameters from Raman spectrum, the $\mathrm{I}(\mathrm{D}) / \mathrm{I}(\mathrm{G})$ ratio and $\mathrm{G}$ peak position (shown in (a) and (b)), as the carbon transforms from graphite to nanocrystalline graphite then further to amorphous carbon and ultimately to tetrahedral amorphous carbon (as shown in (c)). The stage of the as-deposited and implanted DLC films in this work can be identified by comparing their Raman parameters ( $\mathrm{I}(\mathrm{D}) / \mathrm{I}(\mathrm{G})$ ratio and $\mathrm{G}$ peak position) with the three-stage model. Source [10] . . . . . .

5.1 Theoretical predication of elemental and vacancy distribution in Co - DLC films at an implantation fluence of $F=7 \times 10^{16}$ atoms.cm $^{-2}$ by DTRIM. . . . 100

5.2 Predicted Co distribution in DLC at different implantation fluences by DTRIM. As the fluence increases it can be seen that the peak and surface concentration of Co increases and the peak position moves closer towards the surface. . . . 102

5.3 HR-RBS spectrum of Co 1.2. The position at which signals from C, Si and Co should arise if located at surface is marked in the figure. It can be observed that $\mathrm{C}$ is present at the surface and Co is present close to the surface. . . . . 108 
5.4 Screenshots of the SIMNRA program used for data analysis. The experimental setup and raw data of Co 1.2 sample are displayed. . . . . . . . . . . . . 110

5.5 SIMNRA fitting of HR-RBS spectrum of Co 1.2 sample. The figure illustrates the necessity for detailed target construction for HR-RBS. Figure 5.5 (a) shows the data from Co 1.2 sample fit with only 4 layers while figure 5.5 (b) shows the same data fit with 27 layers. It can be seen that detailed target construction can fit the HR-RBS data accurately and it allows extraction of precise details measured by the high-resolution setup. . . . . . . . . . . . . .

5.6 Analysis of HR-RBS spectrum of Co 1.2. The data analysis reveals that the Co distribution for Co 1.2 assumes a Gaussian distribution with a peak concentration of 3.75 at.\% and it does not completely align with the theoretical

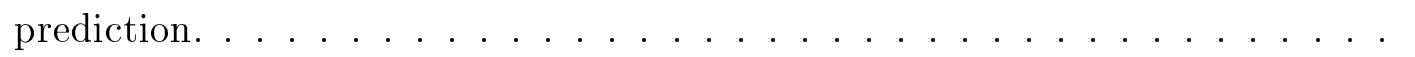

5.7 SIMNRA fitting of Co 2.4 HR-RBS spectrum measuring the bimodal Co dis-

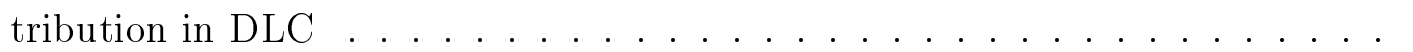

5.8 Analysis of HR-RBS spectrum of Co 2.4. The data analysis reveals that the Co distribution for Co 2.4 assumes a bimodal distribution in contrast to the theoretically predicted unimodal distribution. . . . . . . . . . . .

5.9 SIMNRA fitting of Co 4 HR-RBS spectrum measuring the asymmetric bimodal Co distribution in DLC. . . . . . . . . . . . . . .

5.10 Analysis of HR-RBS spectrum of Co 4. The data analysis reveals preferential displacement of Co towards the surface resulting in an asymmetric bimodal distribution. . . . . . . . . . . . . . . . . . .

5.11 SIMNRA fitting of Co 7 HR-RBS spectrum measuring the enhanced bimodal Co distribution in DLC. . . . . . . . . . . . . . . . 127

5.12 Analysis of HR-RBS spectrum of Co 7. Comparison with DTRIM simulation suggests a major fraction of implanted Co to lie outside the predicted distribution towards the DLC surface. . . . . . . . . . . . .

5.13 SIMNRA fitting of Co 12 HR-RBS spectrum measuring the steady-state distribution of Co implanted into DLC. . . . . . . . . . . . .

5.14 Analysis of Co distribution in Co 12 in comparison with Co distribution in Co 7 and Co distribution predicted by DTRIM. . . . . . . . . . . . 133

5.15 Cross-sectional TEM images of Co implanted DLC showing the evolution of Co distribution and nanoparticle formation with increasing Co implantation fluence in DLC. Right at top of the image is the platinum layer followed by the DLC surface incorporating the implanted Co nanostructures followed by the unimplanted region and the Si interface. . . . . . . . . . . . . . . 137 
5.16 Areal compositional mapping of Co 7 TEM cross-section showing the distribution of different elements within the sample. . . . . . . . . . . . 140

5.17 Line scan 1 of Co implanted DLC showing the distribution of different elements within the TEM lamella. . . . . . . . . . . . . . . . . . . . 142

5.18 Line scan 2 of Co implanted DLC showing the distribution of different elements within the TEM lamella. . . . . . . . . . . . . . . . . . . . 143

5.19 Contrast Analysis of the TEM image of Co 7 sample. The contrast analysis reveals the position of the surface and center peak of the bimodal Co distribution. . . . . . . . . . . . . . . . . 145

5.20 Nanoparticle distribution analyzed from TEM image of sample Co 7 shown in figure 5.15 (c). The nanoparticles are observed to be bimodally distributed in size. . . . . . . . . . . . . . . . . . . . . .

5.21 HR-TEM image of nanoparticles seen in DLC implanted with a Co fluence of $7 \times 10^{16}$ atoms.cm ${ }^{-2}$. The inset shows the Fast Fourier Transform of the HR-TEM image.

5.22 Evolution of bimodal distribution in Co implanted DLC evidenced by HR-RBS and TEM measurements. . . . . . . . . . . . . . . . . .

6.1 Comparison of DTRIM predicted Co implantation profile in a:C and a:C-H films for a fluence of $4 \times 10^{16}$ atoms.cm ${ }^{-2}$. It can be observed that in a:C films the Co peaks about $10 \mathrm{~nm}$ closer to the surface due to the increased stopping. 159

6.2 SIMNRA fitting of amorphous carbon films implanted with Co to a fluence of $4 \times 10^{16}$ atoms.cm ${ }^{-2}$. The fit shows a unimodal Co distribution upon implan-

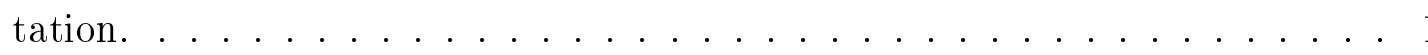

6.3 Analysis of HR-RBS analysis of Co distribution in amorphous carbon films implanted with Co to a fluence of $4 \times 10^{16}$ atoms.cm ${ }^{-2}$. The experimental measurement agrees with the DTRIM prediction on the nature of distribution (unimodal) but the Co peak is measured to be closer to the surface and narrower than the predicted distribution. . . . . . . . . . . 161

6.4 Comparison of Co distribution in Co implanted a:C film and a:C-H films (for a fluence $4 \times 10^{16}$ atoms.cm $\left.{ }^{-2}\right)$. . . . . . . . . . . . . .

6.5 Cross sectional TEM images of amorphous carbon films implanted with Co to a fluence of $4 \times 10^{16}$ atoms.cm ${ }^{-2}$ showing nanoparticle formation. The film surface is marked with white dashed lines.

6.6 Areal composition mapping of amorphous carbon films implanted with Co $\left(4 \times 10^{16}\right.$ atoms.cm $\left.{ }^{-2}\right)$, showing the distribution of Co within the carbon film. 164 
6.7 Hydrogen profile in as-deposited DLC films synthesized by MSIBD discussed in chapter 4. . . . . . . . . . . . . . . . . . . 166

6.8 RNRA profile of Co 0.8 and Co 1.2 showing hydrogen redistribution after Co implantation. Hydrogen depletion is observed in the near-surface region followed by hydrogen accumulation at deeper regions. . . . . . . . . . . 168

6.9 RNRA profile of Co 2.4 and Co 7 showing hydrogen redistribution after Co implantation. Hydrogen depletion is observed to have enhanced in the nearsurface region followed by similar hydrogen accumulation at deeper regions. .

6.10 RNRA profile of hydrogen distribution in DLC film deposited by direct ion beam deposition and implanted with Co to a fluence of $4 \times 10^{16}$ atoms.cm $^{-2}$. It can be observed DIBD deposited DLC films show similar hydrogen redistribution as observed in MSIBD films.

6.11 RNRA profile of hydrogen redistribution in Co 12 sample. The steady-state condition earlier measured for the Co 12 sample is not observed to affect the hydrogen redistribution caused due to Co implantation. . . . . . . . . . . 173

6.12 DTRIM prediction of hydrogen and vacancy distribution in Co implanted DLC films (Co 2.4 sample). . . . . . . . . . . . . . . 176

6.13 Raman Spectrum of as-deposited and Co implanted DLC films (Co 1.2, Co 2.4 and Co 7). The figure shows the spectrum of the as-deposited film fit with D and G peak (double Gaussian fit). Changes in the D and G peak parameters with implantation is used to assess the effects of ion implantation on the structure of DLC film. . . . . . . . . . . . . . . . . . . . 179

$6.14 \mathrm{I}(\mathrm{D}) / \mathrm{I}(\mathrm{G})$ ratio plotted as function of implantation fluence. Ion implantation is observed to increase the $\mathrm{I}(\mathrm{D}) / \mathrm{I}(\mathrm{G})$ ratio in proportion with the increase in implantation fluence. . . . . . . . . . . . . . . . 181

6.15 Raman spectra of implanted and as-deposited a:C film. The figure shows the Raman spectrum of the a:C films fit with D and G peaks. The Raman fit for $\mathrm{a}: \mathrm{C}-\mathrm{H}$ films are shown in the inset for reference. . . . . . . . . . . . .

7.1 Magnetization measurements of Co implanted DLC (Co 1.2, Co 2.4, Co 7, Co 12 at $5 \mathrm{~K}$ ). 7.1 (a) shows the dependence of magnetic moment induced in Co implanted DLC on the applied magnetic field. The magnetic moment is observed to saturate at fields greater than 2 T. 7.1 (b) plots the saturated magnetic moment per Co atom against implantation fluence. It can be seen that the saturated moment increases with the fluence. . . . . . . . . . 187 
7.2 Magnetic moment per Co atom measured as function of applied field at different temperatures for sample Co 7 and Co 12. Both sample show strong temperature dependence uncharacteristic of bulk Co. . . . . . . . . . . 189

7.3 Saturated magnetic moment per Co atom measured against temperature for Co 7 sample at a constant applied field of $6 \mathrm{~T}$. The saturated moment falls exponentially with temperature and is fit with a spin glass model with a characteristic spin freezing temperature of 133 K. . . . . . . . . . . . . 190

7.4 Logarithmic plot of magnetic moment vs measurement temperature for Co 7.192

7.5 Schematic representing crystals - glass / ferromagnets - spin glass. . . . . . 193

7.6 Logarithmic plot of magnetic moment vs measurement temperature for Co 12. The saturated moment deviates from the logarithmic dependence at higher temperature $(>200 \mathrm{~K})$. The spin glass contribution was fit with a freezing temperature of $94 \mathrm{~K} . \ldots \ldots \ldots \ldots$

7.7 Saturated magnetic moment per Co atom measured against temperature for Co 12 sample. The data is fit with a combination of spin glass behavior and Bloch temperature dependence observed in ferromagnets. The spin glass contribution is fit with a spin freezing temperature of $94 \mathrm{~K}$. The Bloch contribution was fit with a spin stiffness coefficient of $1 \times 10^{6}$. . . . . . .

7.8 FFT of HR-TEM images of Co implanted DLC reflecting the structural order present within the nanoparticles. . . . . . . . . . . . .

7.9 ZFC-FC measurement performed on Co 7 at $0.015 \mathrm{~T}$. The inset shows the splitting between the FC and ZFC curves at $4 \mathrm{~K}$ indicating superparamagnetic behavior. . . . . . . . . . . . . . . . . . .

7.10 ZFC-FC measurement performed on Co 12 at $0.015 \mathrm{~T}$. The inset shows the splitting between the FC and ZFC curves. The splitting occurs at a larger temperature than observed for Co 7 indicating superparamagnetism from larger nanoparticles. . . . . . . . . . . . . . . .

7.11 High temperature region of ZFC-FC measurement performed on Co 7 at 0.015 $\mathrm{T}$. The inverse of magnetic moment is plotted against measurement temperature showing the non-Curie dependence of the superparamagnetic nanoparticles. 199

7.12 Resistance measured against temperature for sample Co 12 and Co 7. Both Co 12 and Co 7 show decrease in resistance with increasing temperature. However, the data curve indicates that the temperature dependence is not uniform throughout the temperature range measured. . . . . . . . . . . . 202

7.13 Logarithmic temperature dependence of resistance of Co 12 in the low temperature region. 
7.14 Schematic representing the simplified model of conduction mechanism in Co implanted DLC. The conduction through the implanted DLC region can be split into two parallel conducting chains with tunneling resistance dominating at low temperature region (forming the first chain) and the semiconducting and metallic contribution to conduction at higher temperature region (forming the second parallel chain). . . . . . . . . . . . . . 206

7.15 The dependence of resistance on measurement temperature is fit with the model for parallel network of conduction displayed in figure 7.14. The fit agrees well with the resistance data. . . . . . . . . . . . . . 208

7.16 Logarithmic temperature dependence of resistance of Co 7 in the low temperature region. . . . . . . . . . . . . . . . . 209

7.17 Modeled fit of resistance vs temperature data for Co 7. . . . . . . . . 210 


\section{List of Tables}

2.1 Angular range for collisions $\ldots \ldots \ldots \ldots \ldots$

2.2 Energy transfer during collisions . . . . . . . . . . . . . . . 15

2.3 Reduced energy of different ion beam processes $\ldots \ldots \ldots \ldots . \ldots 17$

3.1 Mass separation by electromagnet in Industrial implanter at GNS Science . . 50

3.2 Experimental parameters for HR-RBS setup . . . . . . . . . . . . 60

4.1 Key ion interaction parameters for DLC deposition . . . . . . . . . . 81

4.2 HR-RBS parameters . . . . . . . . . . . . . . . 86

4.3 Ion species produced by the Penning ion source operating with butane gas . 91

5.1 Ion implantation parameters . . . . . . . . . . . . . . . . . . . . 9 98

5.2 Details of implantation fluence for samples used in this chapter . . . . . . . 98

5.3 Simulation of Co distribution in DLC . . . . . . . . . . . . . . . 104

5.4 Sputtering induced from ion implantation . . . . . . . . . . . . 105

5.5 HR-RBS parameters . . . . . . . . . . . . . . . . . 107

5.6 Kinematics for $500 \mathrm{keV} \mathrm{He}^{+}$beam . . . . . . . . . . . . . . . 107

5.7 SIMNRA target summaries of Co 1.2 HR-RBS spectrum . . . . . . . . . 113

5.8 Fitting parameters of Co 1.2: Experiment vs Theory . . . . . . . . . 117

5.9 Co 2.4 SIMnRA Target composition _ . . . . . . . . . . . . . . . 119

5.10 Co 2.4 Experiment vs Theory . . . . . . . . . . . . . . . . . . . . . . 122

5.11 Co 4 SIMnRA Target composition . . . . . . . . . . . . . . . . . 124

5.12 Co 4 Experiment vs Theory . . . . . . . . . . . . . . . . . . 126

5.13 Co 7 SIMNRA Target composition _. . . . . . . . . . . . . 128

5.14 Co 7 Experiment vs Theory . . . . . . . . . . . . . . . . . 130

5.15 Co 12 SIMNRA Target composition . . . . . . . . . . . . . . . . . 132

5.16 Line scan details - STEM . . . . . . . . . . . . . . . . . . . 144

5.17 HR-RBS - TEM comparison . . . . . . . . . . . . . . . . 146 
6.1 Samples measured by RNRA . . . . . . . . . . . . . . . . . 167

6.2 RNRA profile of Co implanted DLC . . . . . . . . . . . . . . . . 174

6.3 Fitting parameters of Raman spectra of as-deposited and implanted a:C-H films 180

6.4 Fitting parameters of Raman spectra of as-deposited and implanted a:C and

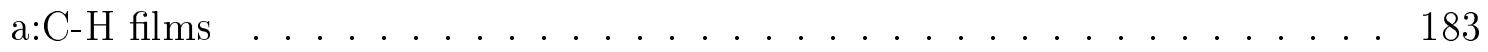

7.1 Fitting parameters for Co 12 transport measurements . . . . . . . . . . 208

7.2 Fitting parameters for Co 7 transport measurements . . . . . . . . . . 211 



\section{Chapter 1}

\section{Introduction}

This research is motivated by the unique effects and potential applications associated with magnetic ion implantation into diamond-like carbon. The thesis is aimed towards answering the question "What happens when Co ions are implanted into diamond-like carbon?". This question is answered in terms of the changes induced in the structure, composition, magnetic behavior and electrical conductivity of DLC during ion implantation. Studying these changes provides a deep insight on the exotic ion-solid interactions happening between the energetic Co ions and the insulating DLC matrix. It further enables analyzing their subsequent effects on various properties of DLC. This study can lead to interesting ways to synthesize novel magnetic nanomaterials.

Diamond-like carbon is an allotrope of carbon with significant fraction of its carbon bonded in $\mathrm{sp}^{3}$ hybridization [8]. The $\mathrm{sp}^{3}$ bonded carbon imparts it with high mechanical hardness and wear resistance [8]. It is a common practice to incorporate hydrogen within the diamond-like carbon matrix to enhance its $\mathrm{sp}^{3}$ content [8]. This material is widely used in the coating industry for its excellent mechanical properties and chemical stability. In this research hydrogenated diamond-like carbon (DLC) is used as the base material for implantation work. Ion implantation is a process in which energetic ions are bombarded into a target surface to modify its surface properties. It is a precise technique widely used in the semiconducting industry to dope Si wafers with $\mathrm{n}$ or $\mathrm{p}$ type impurities [11]. The dopant concentration and distribution can be precisely controlled by selecting the implantation energy and dose [12]. In this work, low energy ion implantation ${ }^{1}$ (30 keV) was used to incorporate Co ions into DLC matrix.

There are two major objectives of this research work. The first objective is to investigate the structural changes induced by low energy ion implantation in DLC. A chief

\footnotetext{
${ }^{1}$ Low energy implantation in general refer to any implantation work carried out in the range of few $\mathrm{keV}$ to few 10 's of keV
} 
outcome of this investigation would be an improved understanding of ion-solid interactions in low energy implantation of transition metal ions into a thermally insulating target composed of light atomic elements. It is hypothesized that implantation into light elements under thermal insulation can lead to manifestation of non-ballistic effects resulting from collision cascades and thermal spikes [13, 14]. There have been very few reports on experimental investigations of such effects in low energy conditions [15, 14, 16, 17]. Utilization of these effects can not only improve the accuracy of the process but can also lead to novel ways of fabricating nanomaterials for a wide range of applications. Diamond-like carbon is an excellent host material satisfying the above criteria for study on the non-ballistic effects of low energy ion implantation.

The second objective is motivated by the intense search for novel magnetic nanostructures and semiconductors for realizing active spintronic devices [18, 19]. 'Spin' is a fundamental property of all particles. It refers to a particle's intrinsic angular momentum. There is a significant interest in synthesizing nanomaterials that can control the spin degree of freedom of its charge carriers [18, 19]. Current technologies are based on transport of charges within the device architecture. Incorporation of nanomaterials that can exhibit spin polarization and control spin transport in a device architecture will lead to fast, powerful and energy efficient technologies [18, 19]. This field of technology based on the property of spin of is known as spintronics.

Electrons (and holes) are the mobile carriers of charge and spin in materials. Thus transport of charges from a spin polarized material would ultimately result in transport of spins. A magnet is a material that can exhibit a significant net spin polarization in its mobile charge carriers. However, a material needs be semiconducting in nature to establish control over transport of charges. Thus the requirement is a magnetic semiconductor (MS) which is a material that can integrate magnetic order with semiconducting properties. There are two types of magnetic semiconductors: (a) condensed magnetic semiconductors (CMS) where the magnetic ions are concentrated as nanoparticles embedded in a semiconducting matrix and (b) dilute magnetic semiconductors (DMS) where the magnetic ions are uniformly distributed throughout the matrix [20]. CMS are interesting as they can show high linear magnetoresistance which is useful for sensing and data storage applications [21]. Realization of DMS can lead to an efficient spin injector for operation of spin transistors and is hence pursued with great interest [20].

The most widely used approach to fabricate MS is to synthesize compound semiconductors with a small fraction of its cations replaced by magnetic ions. These magnetic impurities are theorized to contribute spin polarized electrons in the conduction band thereby resulting in charge carrier mediated ferromagnetic order [22]. Some popular examples are (Mn,Ga)As 
[23], (Co,Ti) $\mathrm{O}_{2}$ [24] and (Fe,Sn) $\mathrm{O}_{2}$ [25]. Of the given examples, (Mn,Ga)As is the most studied material that is known to incorporate intrinsic magnetic order in the semiconducting matrix. However, (Mn,Ga)As does not exhibit magnetic order at room temperature [23]. Of those materials that exhibit room temperature magnetic order, the origin of magnetic order is unclear [23]. Some studies suggest the magnetic order to arise from magnetic nanoparticles [26, 27]. For application as DMS, it is necessary for the magnetism to arise intrinsically from the doped material.

Currently different methods such as atomic layer deposition [28], chemical vapor deposition [29], and molecular beam epitaxy [30] are employed to achieve magnetic semiconductors operable at room temperature. However, fabrication of such compound semiconductors requires complex processing steps and accurate control over the dopant concentration, charge carrier concentration and crystalline structure. Scaling these technologies to industrial scale can itself limit the application of these materials. This research can lead to synthesis of novel magnetic materials and may even establish a fabrication protocol that can help in realizing spintronic applications worldwide. The method and base material of our choice are ion implantation and diamond-like carbon respectively. The justification for their use is as follows,

Method: Ion implantation is an already well established technique in the IC manufacturing industry [11, 12]. Apart from the precise control offered by ion implantation, the process involves interactions between energetic ions with the base matrix at nonthermal equilibrium conditions. This can potentially overcome the solubility limits of the dopant ions imposed by the base matrix and can lead to synthesis of DMS [31]. On the other hand, several recent reports indicate that incorporation of high concentration of magnetic dopants into a dielectric medium by the process of ion implantation results in formation of nanoparticles with core-shell structures. These nanostructures exhibit several interesting properties such as spin polarization with high magnetoresistance [32], magneto-electric coupling [33] and superparamagnetism [34]. Thus ion implantation can also be a useful tool for synthesizing CMS.

Material: Diamond-like carbon is used in several industrial applications as protective surface coatings. One prominent example is in the fabrication of magnetic hard disks where a thin layer of DLC is coated on top of the magnetic layer to protect it from scratches and wear [35]. Unlike compound semiconductors DLC fabrication can be relatively simple and inexpensive $[3,36,37]$. Hence it is a suitable industrial candidate for MS fabrication. Moreover it offers great compatibility with ion implantation. Ion implantation generally suffers from the drawback of destroying the crystal lattice of 
base matrix upon high dose implantation. However, in the case of DLC, ion implantation increases their electrical conductivity by enhancing their $\mathrm{sp}^{2}$ character which makes them more compatible with electronics device fabrication [38]. Lastly, there is a great interest to use amorphous oxide materials as host for magnetic doping for MS applications [39]. There has been less than 5 papers exploring magnetic doping of DLC and no paper so far on magnetic ion implantation into DLC to the best of our knowledge. DLC can thus be an interesting host for magnetic ion implantation.

The choice of Co ions for implantation is based on the studies that have incorporated Co in a semiconducting matrix, and especially in diamond lattice to fabricate magnetic semiconductors [40, 41, 42, 27]. This research work records the investigation of the magnetic and transport properties of Co implanted DLC for the first time in literature.

In order to understand and explain the measured magnetic and transport properties it is important to know about the distribution, configuration and the overall effect of the implanted ions in the DLC matrix. This again leads back to the primary objective of this research which is to explore the structural effects of low energy ion implantation in diamondlike carbon. Diamond-like carbon is an excellent thermal insulator [43]. There have been few early reports measuring unique implantation profiles upon ion implantation in thermal insulators. These profiles were bimodal in nature and did not match the predictions based on the ballistics of ion implantation. They were explained by the effects of diffusion due to thermal spikes [14], charging effects observed due to use of high current density [16] and stress incorporated during ion implantation [17]. However, a thorough study on the evolution of such distribution is lacking and no conditions are established so far regarding the onset of such effects. The earlier studies were focused on eliminating such effects observed during implantation since they compromised with the precision offered by the implantation technique.

But these effects can be interesting for a number of applications. For example, ion implantation by its very nature imparts a non-homogeneous dopant distribution within the base matrix. In order to uniformly distribute Co within DLC it would be ideal if the unimodal distribution can be broadened by effects of diffusion. Annealing which is commonly used to cause such diffusion requires an additional processing step and leads to exposure of the material to high temperature which may not be advantageous in all cases. Thus exploration of effects not predicted by ballistics of the implantation may lead to new methods of dopant distribution and is thus of interest to the ion beam community. This research work experimentally investigates these effects for the first time at low energy implantation conditions and provides a model explaining the evolution of bimodal distribution in thermal insulators. 
The thesis is structured in 8 chapters. The motivation and key objectives of the research work are covered so far.

Chapter 2 explains the basic concepts and theory underlying the different components of the research. The chapter covers in detail the basic concepts of ion-solid interactions. Once the key factors are discussed, their application in the form of ion beam deposition, implantation and ion beam analysis are explained. This is followed by an overview of the theoretical layout encompassing some basic magnetic properties of materials. These concepts are relevant in explaining the magnetic behavior of ion implanted DLC.

Chapter 3 covers the experimental methods and methodology employed in this research. Some of the key techniques used for synthesis of DLC is direct ion beam deposition and mass-selective ion beam deposition. Both the systems are explained in detail in this chapter. A system similar to the one used for mass-selective ion beam deposition is used for ion implantation. The working of the system is described in detail in this section. The next part of the chapter deals with the characterization techniques used in this research. The techniques used are high-resolution Rutherford backscattering, transmission electron microscopy, resonant nuclear reaction analysis, Raman spectroscopy, magnetic and physical property measurements. The basic principle of working along with the associated experimental uncertainties are provided in this chapter.

Chapter 4 deals with synthesis of DLC films by ion beam deposition. The properties of DLC films synthesized by the techniques: direct ion beam deposition and mass-selective ion beam deposition are discussed with respect to the deposition parameters. The DLC films are primarily analyzed by Raman spectroscopy and Resonant Nuclear Reaction Analysis. Transmission Electron Microscopy and Rutherford backscattering adds further insight to the properties of the film.

Chapter 5 presents the most significant results of the structural investigations carried out on Co implanted DLC films. The chapter begins with an overview of the results predicted from considering the ballistics of the implantation process. For this purpose a simulation software code called DTRIM is utilized. This is followed by presenting the results obtained from high resolution Rutherford backscattering. The measured ion distribution were evaluated as a function of implantation dose and was observed to evolve to bimodal distribution at higher doses. These films were further analyzed with the help of images from cross-sectional transmission electron microscopy. The information gathered together from these techniques were used to construct a model explaining the measured results.

A key prediction based on the model constructed in chapter 5 is tested out in Chapter 6. This chapter basically deals with the influence of hydrogen on the implanted Co distribution and vice versa. DLC films synthesized with and without hydrogen in the base 
matrix are implanted with Co at same conditions to same implantation fluence. The ion distribution and composition measured from these films are compared against each other and the model presented in the previous chapter is verified. The chapter further continues on the structural investigation of ion implanted DLC films by measuring the redistribution of hydrogen upon ion implantation by resonant nuclear reaction analysis. The last part of this chapter focuses on the effect of ion implantation on the overall DLC structure making use of Raman spectroscopy to analyze the disorder, clustering and re-hybridization induced in DLC during ion implantation.

Chapter 7 covers the magnetic and transport properties of ion implanted DLC. Results from the measurements of Co implanted DLC films by magnetic property measurement system and physical property measurement system forms the crux of this chapter. Co implanted DLC exhibits room temperature magnetic order and strong dependence on implantation fluence and measurement temperature. The conductivity of the implanted films were measured against changing temperature. The magnetic behavior and transport properties of ion implanted DLC are explained from the perspective enabled by the structural investigation of DLC. The chapter also leads to areas for future research on ion implanted DLC.

Chapter 8 concludes the research work by summarizing the results obtained so far and putting them in an overall perspective. Chapter 8 also covers the outlook of the research on ion implantation in DLC films. 


\section{Chapter 2}

\section{Background And Theory}

This chapter provides the theoretical background for the research carried out in this thesis. The chapter is divided into two sections. Since this thesis primarily focuses on ion beam techniques, the first section explains the different concepts associated with ion-solid interactions and their implications on ion beam deposition, ion implantation and ion beam analysis. The second section deals with the magnetic properties of materials. The concepts covered in this section are later used to explain and compare the results obtained from the magnetic and electrical measurements on Co implanted DLC films.

\section{$2.1 \quad$ Ion-solid Interactions}

The primary aim of this research is to investigate the effects of ion beam process on DLC. DLC is synthesized by high energy ion beam deposition. The term "high energy" has to be interpreted in the context of deposition process. In this work, molecular ion beams with energy as high as $10000 \mathrm{eV}$ were used to synthesis DLC films. Deposition of thin films at this energy range is not possible for most of the materials. DLC is a special case where such deposition is possible due to preferential sputtering of silicon (substrate) and low sputter yield of carbon. Utilization of this process requires an understanding of the sputtering process.

One of the biggest advantage offered by ion implantation is its precise control over the range and distribution of impurities in a material. These parameters are governed and hence can be evaluated by considering the ballistics of ion solid interactions. This research ventures into an area where non ballistic effects can play a significant role. Analyzing these effects requires quantitative interpretation of the ion implantation process. Moreover, efficient interpretation of data obtained from HR-RBS and RNRA requires a firm understanding and quantitative evaluation of the cross section of collisions and ion stopping in the target material. Hence, various concepts of ion solid interactions required to efficiently utilize the above 
processes are discussed in sufficient detail in this section.

An ion is defined as an atom or a molecule that has a net electric charge due to loss or gain of one or more electrons. The presence of an electric charge allows it to be controlled by an electric field. For example, an ion can be accelerated, decelerated, focused, steered or scanned over an area merely by application of appropriate electric field. In an ion beam equipment, ions are produced in an ion source and are accelerated onto a target surface. The energy of the ion depends entirely on its mass and velocity. These ions based on their energy, atomic number and mass can be used to sputter, deposit, sub-plant, implant, radiate or characterize a material. The function it performs is dependent on the nature of interaction between the ion and the target material.

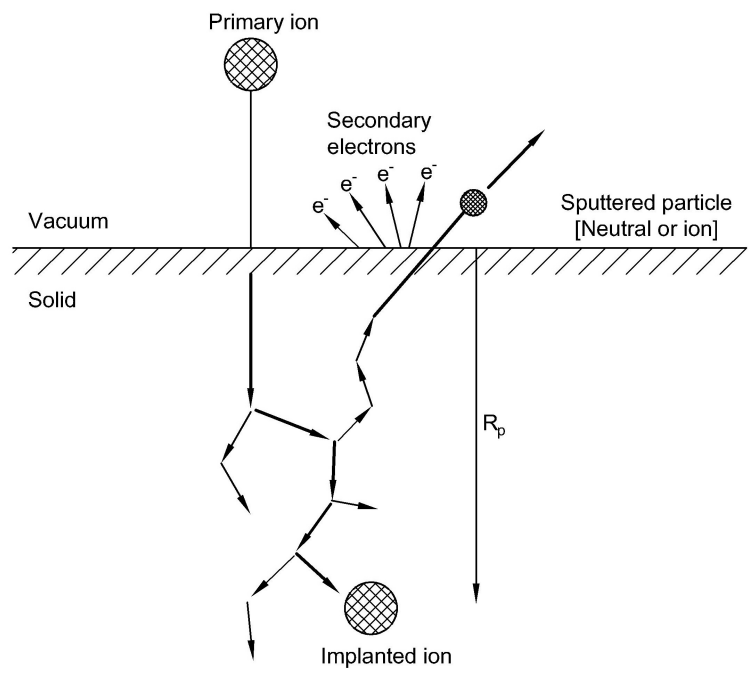

Figure 2.1: Schematic of ion interactions with matter. An energetic ion bombarding on a material surface reaches an average distance $R_{p}$ by loss of energy due to atomic collisions and electronics excitations. Redrawn from [1]

Figure 2.1 shows a schematic of the interaction of an energetic ion with matter. The different concepts required to explain the above process are

1. Interatomic potentials - It denotes to the forces governing the interaction of an energetic ion with atoms of a solid

2. Dynamics of binary elastic collisions - Essential to understand the scattering process and loss of energy of an ion undergoing an elastic collision with a target atom

3. Cross-section - Explains the likelihood for an ion-solid interaction to occur for different ion and target masses, scattering geometry and ion energies.

4. Ion stopping - Refers to the fundamental process of slowing down of ions in matter 
5. Ion range and distribution - It is generally the most useful parameter in ion beam techniques which determines the extent an ion interacts with matter in terms of depth from its surface.

6. Atomic displacements and sputtering - The energy incorporated by the ion into the solid matrix results in atomic displacements and damage which can also lead to sputtering effects

Each of these concepts are described briefly in this section. A detailed explanation can be found in [1].

\subsubsection{Interatomic potentials}

The nature and manner in which an energetic ion interacts with the atoms of a solid can best be described in terms of interatomic forces governing the solid. In a solid, these forces can either be attractive or repulsive depending on the distance separating the neighboring atoms of a solid. The attractive forces originate either from the weak Van der Waals forces or from the forces involved in formation of chemical bonds. The attractive forces are responsible for keeping the atoms in condensed state of matter. The repulsive forces on the other hand originate predominantly from the Coulombic forces between the positively charged nuclei of the neighboring atoms.

These forces are usually expressed in terms of interatomic potential, $V(r)$, which is given by,

$$
F(r)=-\frac{d}{d r}[V(r)]
$$

where $F(r)$ represents the net interatomic force acting on the particles, and $r$ refers to the distance of separation between the particles. A simple numerical model describing the interatomic potential in condensed forms of matter constituting the repulsive and attractive forces is Lennard-Jones potential [44].

$$
V(r)=\varepsilon\left[\left(\frac{\Gamma_{0}}{r}\right)^{m}-\left(\frac{\Gamma_{0}}{r}\right)^{n}\right]
$$

where $\varepsilon$ represents the minimum energy (negative potential energy) possessed by the atom at its equilibrium interatomic distance, $\Gamma_{0}$ represents the interatomic distance at which the interatomic potential is $0, m$ is a power factor describing the strength of the Pauli's repulsion and $n$ is a power factor describing the strength of long range attraction. $m$ and $n$ typically have a value of 12 and 6 respectively [44]. Such a potential form is represented in figure 2.2. 
Figure 2.2 also shows a simple schematic showing the effect of interatomic forces between two neighboring atoms. The equilibrium distance at which atoms assume their lowest energy state is denoted by $r_{0}$. It can be seen that, if an atom has to be displaced from its equilibrium distance it requires excess energy as defined by the potential. Thus the curvature of the potential describes how easy (or difficult) it is to shift an atom from its equilibrium distance which is commonly referred to as its elasticity and the depth of the curve denotes the energy required to remove the atom from the solid which defines its binding energy. The binding energy is an important term for evaluating the damage and sputtering induced by the ion beam process.

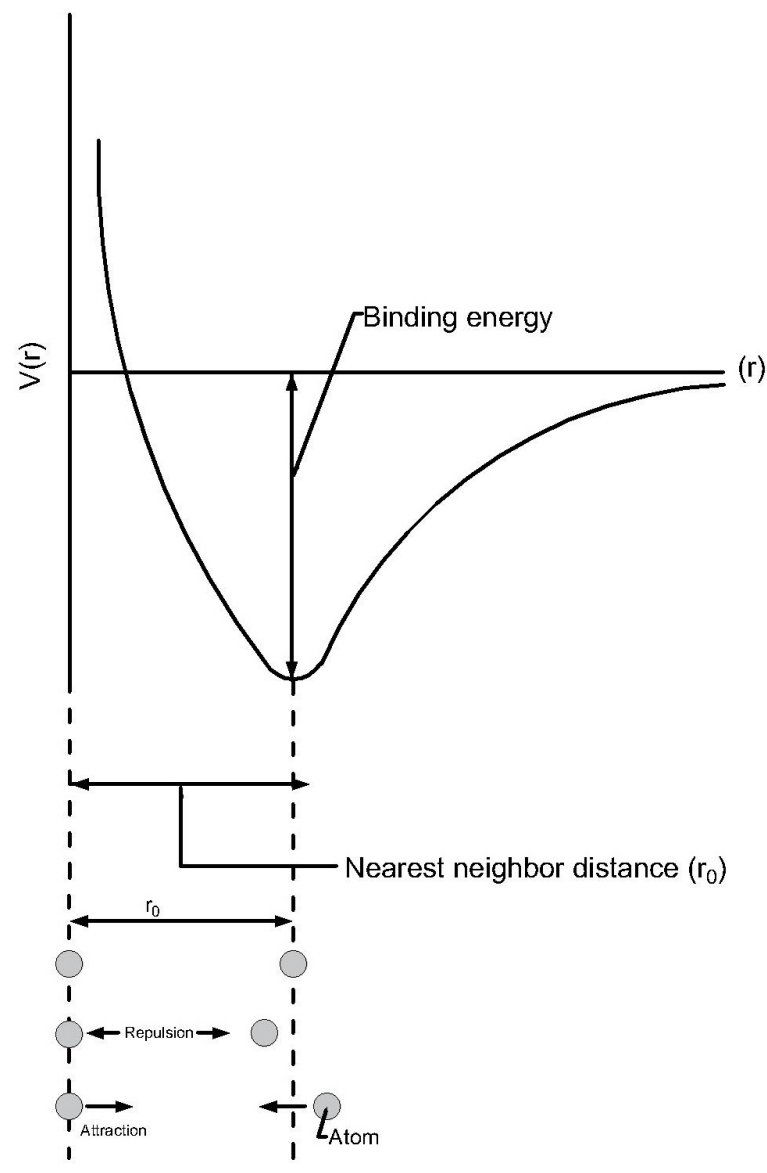

Figure 2.2: Schematic of Interatomic forces and interatomic potential as a function of interatomic distance. As an atom moves away from its equilibrium position it experiences a repulsive or an attractive force to reinstate its equilibrium position and its strength depends on the interatomic potential between its neighboring atoms. Redrawn from [1]

At room temperature, thermal energy is not sufficient to cause significant displacement of the atom from its equilibrium state. However, in the case of collision with energetic ions, 
the ion can approach very close to the nucleus of the atom. At such close distances, the repulsive force experienced by the ion can be given by

$$
V(r)=\frac{Z_{1} Z_{2} e^{2}}{r} x(r)
$$

where $x(r)$ is the screening function that describes the shielding of the Coulomb interaction by the electron cloud surrounding the nucleus, $Z_{1}$ and $Z_{2}$ represents the atomic number of the ion and the target atom respectively and $e^{2}=1.44 \mathrm{eV} . \mathrm{nm}$ (cgs units). The screening function is defined as the ratio of the actual atomic potential, at some radius $r$, to the Coulomb potential [1]. In ideal condition $x(r)$ can be used to describe the interaction between the ion and the atom at all interatomic distances. For example, when $r<<a_{0}$, where $a_{0}$ denotes the Bohr radius of the hydrogen atom, $x(r)$ should tend to unity thus describing the interaction purely by Coulomb potential between the two nuclei. When $r>>r_{0}, x(r)$ should tend to 0 reflecting complete shielding of the nuclei by electron giving rise to charge neutrality.

J. F. Ziegler, J. P. Biersack and U. Littmark [45] has arrived at a universal expression for the screening function based on solid state numerical equations of 261 different pairs of atoms. In this model, the total interatomic potential was taken as,

$$
V=V_{n m}+V_{e n}+V_{e e}+V_{k}+V_{a}
$$

where $V$ refers to total interatomic potential, $V_{n m}$ refers to the electrostatic potential energy between the nuclei, $V_{e n}$ refers to the interaction energy between each nucleus and the other electron distribution, $V_{e e}$ refers to the pure electrostatic interaction energy between the two electron distributions, $V_{k}$ refers to the increase in kinetic energy of the electrons in the overlap region due to Pauli excitation and $V_{a}$ refers to the increase in exchange of these electrons. The Coulomb interaction $V_{c}$, which is of interest for our application is given by,

$$
V_{c}(r)=V_{n m}+V_{e n}+V_{e e}
$$

Based on the numerical calculations an expression for a universal screening function $\chi_{U}$ was obtained to be,

$$
\chi_{U}=0.1818 \exp (-3.2 x)+0.5099 \exp (-0.9423 x)+0.2802 \exp (-0.4028 x)+0.02817 \exp (-0.2016)
$$

where $x$ is the reduced distance given by 


$$
x=\frac{r}{a_{U}}
$$

and $a_{U}$ is the universal screening length which is defined as

$$
a_{U}=\frac{0.8854 a_{0}}{\left(Z_{1}^{0.23}+Z_{2}^{0.23}\right)}
$$

The expression is considered to be universal as it can be applied to any set of atoms by considering just their atomic number to obtain a reasonable approximation of the screening function.

The Coulombic repulsion of the bombarding ion by the target atom can be interpreted as an elastic collision between the ion with the target atom defined by the interatomic potential. This forms the basis for development of expressions for ion range and damage in solids. The next subsection covers the dynamics of this elastic collision between ions and atoms which play a crucial part in any ion beam technique.

\subsubsection{Dynamics of binary elastic collisions}

As an energetic ion enters a target material, it undergoes collisions with the stationary atoms which deflects it from its original path. These collisions result in loss of its energy in discrete steps by transferring energy and momentum partially to the recoiling atoms. In order to quantify this process, the collision between the ions and the atoms are treated classically as two-body collisions, considering only the asymptotic values of momentum at great distances from the collision. The principle of conservation of energy and momentum are all that is required to obtain recoil energy as a function of the scattering angle.

There are few assumptions underlying this scattering process. These assumptions as laid out in [46] are,

(a) two-atoms collisions are only considered

(b) classical dynamics is applied

(c) excitation or ionization of electrons only enters as a source of energy loss, but does not influence the collision dynamics

(d) one of two colliding atoms is initially at rest

Now based on these assumptions, consider a collision of an ion with a target atom as seen in the laboratory frame of reference shown in figure 2.3 . 


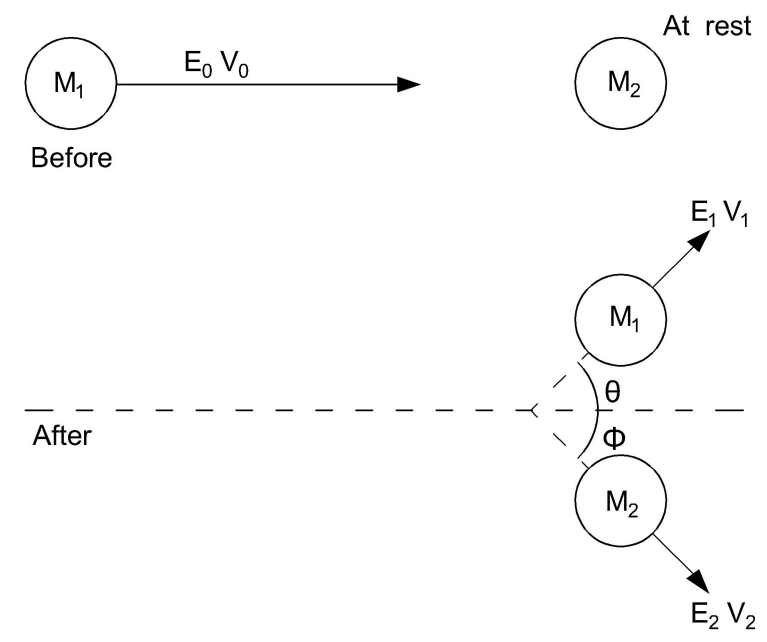

Figure 2.3: Schematic representing binary elastic collision in laboratory frame of reference. An projectile atom of mass $M_{1}$ with initial energy $E_{0}$ and initial velocity $v_{0}$ collides with a stationary atom of mass $M_{1}$ resulting in transfer of energy and momentum. Immediately after collision the projectile atom and the recoiled atom are noted to move in different directions with energy $E_{1}$ and $E_{2}$ and velocity $v_{1}$ and $v_{2}$ respectively. Redrawn from [1]

Consider the collision process in laboratory frame of reference. Application of conservation of momentum and energy results in the following expression [1],

$$
\frac{v_{1}}{v_{0}}=\frac{M_{1}}{M_{1}+M_{2}} \cos \theta \pm\left[\left(\frac{M_{1}}{M_{1}+M_{2}}\right)^{2} \cos ^{2} \theta+\frac{M_{2}-M_{1}}{M_{1}+M_{2}}\right]^{1 / 2}
$$

where $v_{1}$ is the velocity of the scattered projectile in laboratory coordinates, $v_{0}$ is the velocity of the incident projectile in laboratory coordinates, $M_{1}$ is the mass of the projectile and $M_{2}$ is the mass of the target atom, $\theta$ is the scattering angle.

The equation above defines the ratio of the projectile velocity after collision to before collision as a function of scattering angle. Consider the two possible scenario, $M_{1}>M_{2}$ and $M_{1}<M_{2}$. In the first case, the term in the brackets of the equation, $\left(\frac{M_{1}}{M_{1}+M_{2}}\right)^{2} \cos ^{2} \theta+\frac{M_{2}-M_{1}}{M_{1}+M_{2}}$, will equate to zero when,

$$
\cos ^{2} \theta_{m}=1-\frac{M_{2}^{2}}{M_{1}^{2}}, 0 \leqslant \theta_{m} \leqslant \frac{\pi}{2}
$$

where $\theta=\theta_{m}$ denote the maximum scattering angle possible during the collision. For values of $\theta>\theta_{m}$, the ratio of $\frac{v_{1}}{v_{0}}$ is either imaginary or negative. Such a value do not have any physical meaning since $v_{1}$ and $v_{0}$ both refers to the magnitude of the velocity vector. Thus the expression defines the maximum scattering angle possible during the collision of an ion with a target atom, when the ion mass is greater than the target atom. When $M_{1}=M_{2}$, the maximum scattering angle increases to $\frac{\pi}{2}$. Application of this maximum scattering angle 
also allows calculation of the final velocity and thus energy of the ion after collision provided there is knowledge about its initial energy before collision.

The above expressions are significant in ion beam analysis. Consider for example, the RBS analysis of DLC films. The beam incident on the sample is composed of $\mathrm{He}^{+}$ions. The maximum angle by which the helium ion can scatter after collision with hydrogen atom is $14.47^{\circ}$.

The general scattering angles for RBS is usually greater than $90^{\circ}$ [47]. In this work for HR-RBS, the detector is placed at an angle of $65^{\circ}$. Thus in both cases, hydrogen from the sample cannot be probed. This is one of the main reason for opting resonant nuclear reaction analysis in addition to HR-RBS. RNRA provides an accurate depth profile of hydrogen in samples while HR-RBS provides elemental depth profiles for all atoms with mass greater than hydrogen.

For condition $M_{1}<M_{2}$ all values of $\theta$ from 0 to $\pi$ are possible. Since only positive values of the $\frac{v_{1}}{v_{0}}$ are possible, the expression condenses to,

$$
\frac{E_{1}}{E_{0}}=\left[\frac{\left(M_{2}^{2}-M_{1}^{2} \sin ^{2} \theta\right)^{1 / 2}+M_{1} \cos \theta}{M_{2}+M_{1}}\right]^{2}
$$

where $E_{1}$ refers to energy of the ion after collision, $E_{0}$ refers to its energy before collision.

The ratio of final energy to the initial energy of collision is known as the kinematic factor. For example, consider the above expression for application in HR-RBS to analyze the elemental distribution of Co. The kinematic factor for Co for a $65^{\circ}$ scattering angle is 0.925. This implies that when a $500 \mathrm{keV}$ beam hits the sample surface, if Co is present in the surface a signal should be seen arising from $462.3 \mathrm{keV}(500 * 0.925)$. The kinematic factor thus plays a key role in analyzing RBS spectrum. In depth analysis of the as-deposited and implanted DLC films are presented in chapter 4,5 and 6. 

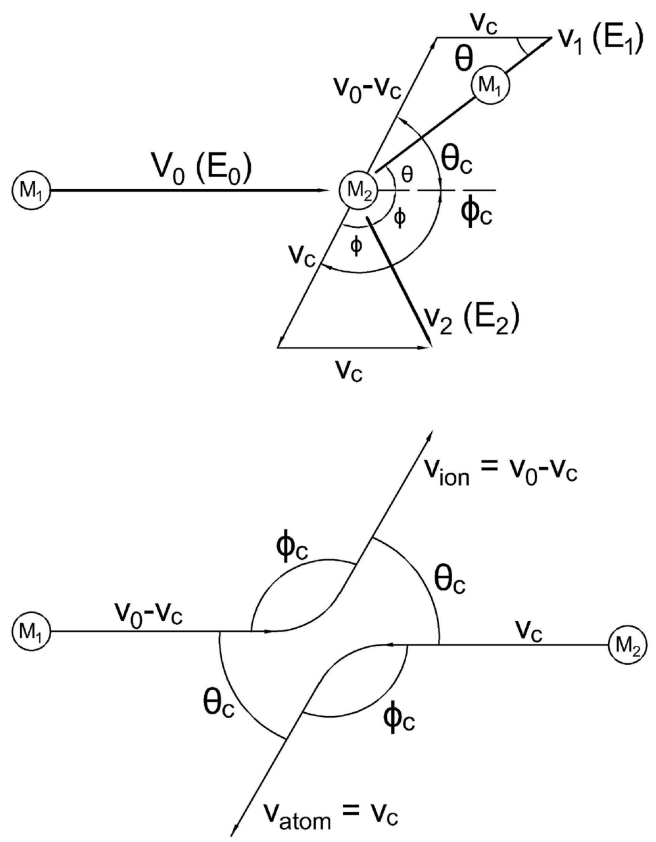

Figure 2.4: Schematic representation of binary elastic collision in laboratory frame of reference and center-of-mass frame of reference. Redrawn from [1]

Figure 2.4 represents the collision process in both laboratory and center-of-mass frame of reference. Application of CM coordinates simplifies calculating the different parameters during the collision process. The angular range and energy transfer process for different target combinations are represented in table 2.1 and 2.2 .

Table 2.1: Angular range for collisions

\begin{tabular}{|c|c|}
\hline Heavy target & $M_{1} \ll M_{2} ; 0 \leqslant\left|\theta_{c}\right|<\pi$ \\
\hline Equal masses & $M_{1}=M_{2} ; 0 \leqslant\left|\theta_{c}\right|<\frac{\pi}{2}$ \\
\hline Light target & $M_{1} \gg M_{2} ; 0 \leqslant\left|\theta_{c}\right| \leqslant \tan ^{-1}\left(M_{1} / M_{2}\right)<\frac{\pi}{2}$ \\
\hline
\end{tabular}

Table 2.2: Energy transfer during collisions

\begin{tabular}{|c|c|}
\hline Heavy target & $\frac{T}{E_{0}} \cong \frac{2}{\pi}\left(1-\cos \theta_{c}\right)$ \\
\hline Equal masses & $\frac{T}{E_{0}} \cong \sin ^{2} \theta_{c}$ \\
\hline Light target & $\frac{T}{E_{0}} \cong \frac{M_{1}}{M_{2}} \theta_{c}^{2}$ \\
\hline
\end{tabular}

An additional parameter that is useful in calculation of various factors of collisional process is the impact parameter. Impact parameter is defined as the perpendicular distance between the path of the projectile and center of the potential field of the stationary atom of the solid as is illustrated in figure 2.5 [1]. 


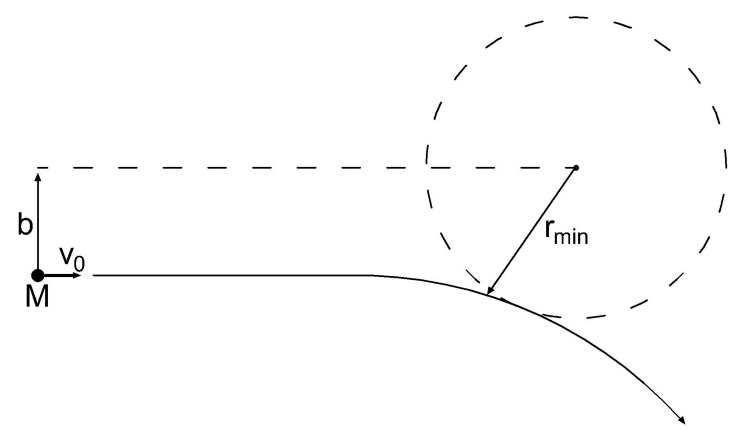

Figure 2.5: Schematic representing collision trajectory and impact parameter. The impact parameter $b$ as represented in the figure is the perpendicular offset between the collision trajectory of the projectile atom and a parallel line passing through the center of mass of the target atom. It can be seen that the impact parameter and minimum distance of separation during collisional process are inter-related. Redrawn from [1]

The interaction of the target atom with the energetic ion can be taken to arise from a central force originating from the position of the target atom. A central force possess circular symmetry. The interaction would thus depend purely on the distance separating the ion and the target atom. The impact parameter, separation distance and energy of the ion (in CM frame) can be related to each other as [1],

$$
b=r_{\min }\left[1-\frac{V\left(r_{\text {min }}\right)}{E_{c}}\right]^{1 / 2}
$$

The main advantage of use of CM coordinate lies in obtaining an expression for the scattering angle as a function of impact parameter.

The scattering angle of the collision in the CM frame is determined by the following expression,

$$
\theta_{c}=\pi-2 b \int_{r_{\text {min }}}^{\infty} \frac{d r}{r^{2}\left[1-\frac{V(r)}{E_{c}}-\left(\frac{b}{r}\right)^{2}\right]^{1 / 2}}
$$

where $\theta_{c}$ and $E_{c}$ refers to the scattering angle and energy in the CM frame. The scattering angle is obtained by the integrating the expression within the bounds of $r$ approaching infinity and $r$ approaching $r_{\min }$ (application of limits). Detailed explanation can be found at [1].

It can be clearly seen that the scattering angle is dependent on the impact parameter, incident energy and the interatomic potential.

For a head-on collision, impact parameter $b=0$. Thus the closest distance an ion can approach an atom is [1], 


$$
d_{c} \equiv\left(r_{\text {min }}\right)_{b=0}=\frac{M_{1}+M_{2}}{M_{2} E_{0}} Z_{1} Z_{2} e^{2}=\frac{Z_{1} Z_{2} e^{2}}{E_{c}}
$$

where $d_{c}$ is the collision diameter.

To put this distance in perspective, it is informative to express $d_{c}$ relative to screening distance, $a_{T F}$ (given by Thomas Fermi model [48]),

$$
\varepsilon \equiv \frac{a_{T F}}{d_{c}}=\frac{a_{T F} E_{c}}{Z_{1} Z_{2} e^{2}}=\frac{E}{Z_{1} Z_{2} e^{2}} \frac{a_{T F} M_{2}}{M_{1}+M_{2}}
$$

where $\varepsilon$ is referred to as reduced energy parameter, $a_{T F}$ is Thomas-Fermi screening distance and is given by [48]

$$
a_{T F}=\frac{1}{2}\left(\frac{3 \pi}{4}\right)^{3 / 2} \frac{h^{2}}{m_{e} e^{2} Z^{1 / 3}}=\frac{0.885 a_{0}}{Z^{1 / 3}}
$$

where $m_{e}$ is the mass of an electron, $h$ is the Planck constant.

The reduced energy, $\varepsilon$, is an important parameter. It is a direct measure of the energy and impact of the collision. The advantage in using reduced energy parameter is that it simplifies the effect of different variables involved in a collisional process, such as $Z_{1}, Z_{2}, M_{1}, M_{2}$ and $E$, into a single parameter $\varepsilon$. Reduced energy for the various ion beam processes used in this research are tabulated below,

Table 2.3: Reduced energy of different ion beam processes

\begin{tabular}{|c|c|c|c|}
\hline S.No & Process & Description & $\varepsilon$ \\
\hline \hline 1 & Ion beam deposition & $5 \mathrm{keV}$ C on $\mathrm{Si}$ & 0.40 \\
\hline 2 & Ion Implantation & $30 \mathrm{keV}$ Co on C & 0.26 \\
\hline 3 & HR-RBS & $500 \mathrm{keV} \mathrm{He}$ on Co & 80.19 \\
\hline 4 & RNRA & $6.5 \mathrm{MeV} 15 \mathrm{~N}$ on $\mathrm{H}$ & 798.04 \\
\hline
\end{tabular}

It can be seen how informative the reduced energy parameter is from the above table. The table puts the different ion beam processes of this research in perspective of how energetic the collision is. It is surprising that ion implantation happens to be less energetic than deposition but this clearly shows the effect of the masses of the incident ion and the target atom in the collisional process. It can be seen that the distance of closest approach of the energetic ion with the target atom reduces from $4.6 \times 10^{-2} \mathrm{~nm}$ in the case of ion implantation to $2.48 \times 10^{-5} \mathrm{~nm}$ in RNRA. Bohr radius of hydrogen atom is $5.3 \times 10^{-2} \mathrm{~nm}$ and the radius of a nucleus is in the order of picometers. In the former case of Co implantation, the ion penetrates inside the electronic shells while in the later case, the ${ }^{15} \mathrm{~N}$ ion reaches close to the nucleus of the hydrogen atom thereby resulting in nuclear reactions. 
So far in this section the dynamics of binary elastic collisions were discussed. From this discussion several useful parameters such as the maximum scattering angle, maximum energy transfer, kinematic factor, distance of closest approach and reduced energy were presented. The importance of some of the above parameters were briefly discussed.

\subsubsection{Cross-section}

Ion beam techniques involves bombardment of a target surface with a large number of ions. These ions interact with an even larger number of atoms. Thus the overall process can be described well by statistics and probability. In the last subsection, the dynamics of ion-atom collisions were covered. An important relation presented in table 2.2 shows that the energy transferred to the recoil atom is directly related to the scattering angle. The scattering angle can assume a wide range of values based on the mass of the ion and the target atom. In this subsection, expressions for the probability for an ion to scatter in specific window of scattering angle or the probability that a collision will result in a specific amount of energy transfer to a recoiling atom will be presented. An important parameter called differential cross-section will be utilized to explain the probability of these events. These expressions are important in determining the detection limits in ion beam analysis.

Cross-section in general expresses the likelihood for an interaction to occur. The scattering cross-section, $\sigma$, represents the effective collision area for an atom. Figure 2.6 represents the physical significance of cross-section in an ion-collision event.

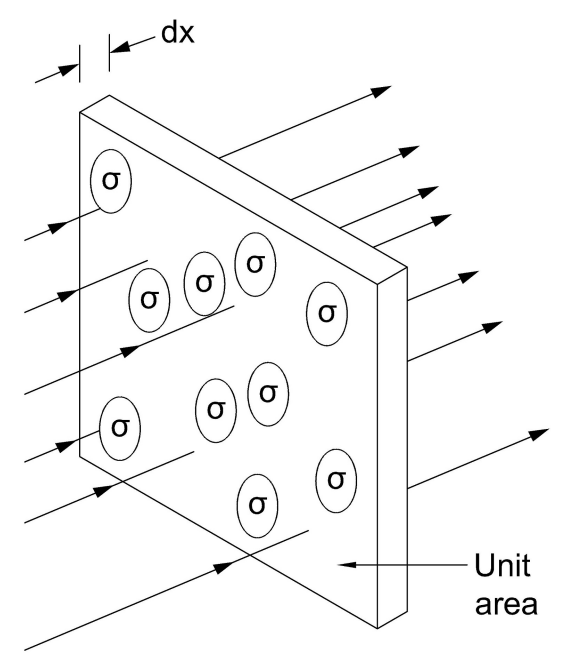

Figure 2.6: Schematic representing the effective cross-section for ion-atom collision in a thin foil. An energetic ion beam impringing a thin foil of unit area and traversing a thickness $d x$ experiences collisions whose number is proportional to the atomic density of the thin foil, the thickness traversed by the ion in the process and the cross-section of the target atoms for collision. Redrawn from [1] 
Consider figure 2.6, where an ion beam traverses a thin target, of thickness $d x$ and unit area containing a total of $N$ atoms per unit volume. The thin target in figure 2.6 contain a total of $N d x$ target nuclei per unit area. If each atom has an effective collision cross-section of $\sigma$, then the probability that an ion collides with an atom is given by,

$$
\text { Probability }=\sigma N d x \text {. }
$$

However, use of ion beam methods require a more specific value. Most often in ion beam methods it is required to compute the cross-section for an ion-atom collision to result in the scattering of the ion within a specific window of scattering angle. Placement of this condition leads to a term called differential cross-section $d \sigma\left(\theta_{c}\right)$ which indicates the probability for an ion to scatter into an angle between $\theta_{c}$ and $\theta_{c}+d \theta_{c}$. The differential cross-section can be related to the impact parameter (figure 2.7) and thus to the scattering angle which is given by [1],

$$
\frac{d \sigma\left(\theta_{c}\right)}{d \theta_{c}}=2 \pi\left(\frac{Z_{1} Z_{2} e^{2}}{2 E_{c}}\right)^{2} \frac{\cos \left(\theta_{c} / 2\right)}{\sin ^{3}\left(\theta_{c} / 2\right)}
$$

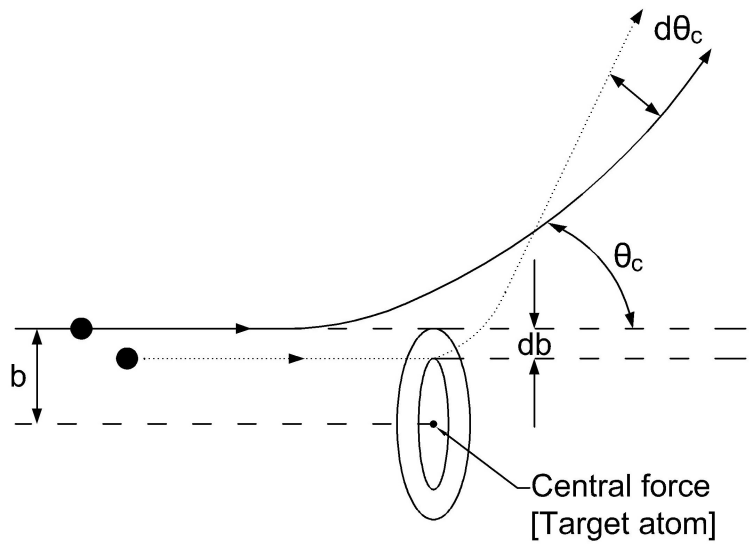

Figure 2.7: Schematic representing dependence of scattering angle on impact parameter. For a projectile atom to be scattered within a specific window of scattering angle it needs to be colliding with the target atom within a specific window of impact parameter. As the impact parameter increases the scattering angle decreases. Redrawn from [1]

This is again a crucial relation for ion beam analysis. The above expression helps in determining the optimum angle at which the detector can be setup. For example, compare placing the RBS detector at a scattering angle of $65^{\circ}$ with $165^{\circ}$. The differential cross section analysis suggests that the probability for $\mathrm{He}^{+}$ion to scatter by $65^{\circ}$ is 40 times greater than scattering by $165^{\circ}$. Thus more signals can be collected by placing the detector at $65^{\circ}$. 
However, this comes at the price of reduced energy resolution during the measurement as the kinematic factor is higher for higher scattering angles.

In ion beam characterization, for example in RBS, the backscattered particles are detected by a detector which has a fixed solid angle, $\triangle \Omega$. Consider a setup shown in figure 2.8 .

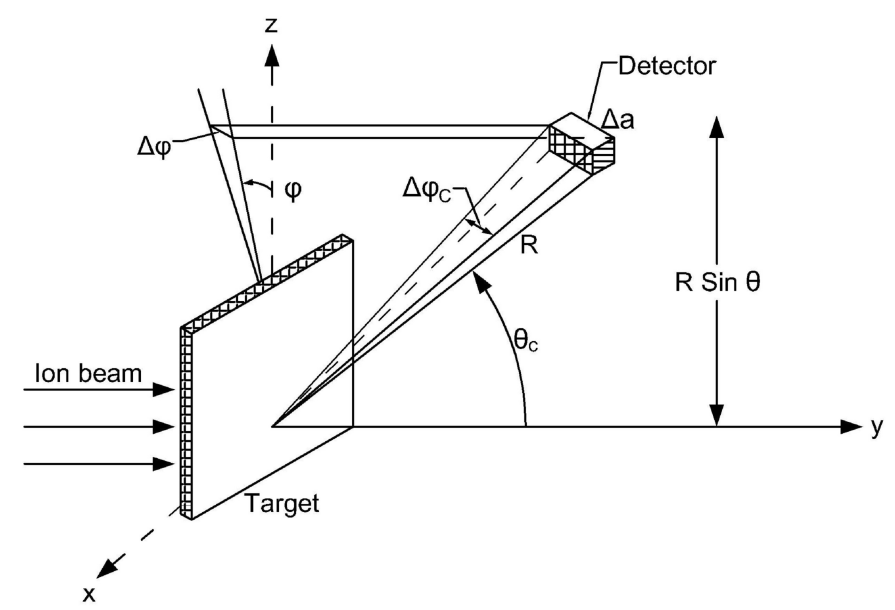

Figure 2.8: Schematic representing a typical ion beam analysis setup where the a detector is placed at a specific position and angle to detect the signal from the ion-atom interaction. The signal can the scattered incident ion beam, the recoiled target atoms or ionizing/electromagnetic radiation released from the collisional process. RBS assumes a similar setup where the detector detects the scattered incident beam at a particular scattering angle and a thus specific solid angle. Redrawn from [1]

The figure shows a beam of ions scattering from a target. The scattered ions are detected by a detector placed at distance $R$ away from the target. The detector measures the ions scattered between the polar angle $\theta_{c}$ and $\theta_{c}+\Delta \theta_{c}$. The solid angle of the detector is given by,

$$
\Delta \Omega=\frac{\Delta a}{R^{2}}=\frac{\left(R \Delta \theta_{c}\right)\left(R \sin \theta_{c} \Delta \varphi\right)}{R^{2}}=\Delta \theta \Delta \varphi \sin \theta_{c}
$$

where $\Delta \Omega$ is the solid angle of the detector, $\Delta a$ is the area of the detector, $R$ is the distance of the sample from the detector

For practical applications it is thus useful to calculate the differential cross-section as a function of solid angle. Figure 2.9 shows the physical significance of such a differential cross-section. 


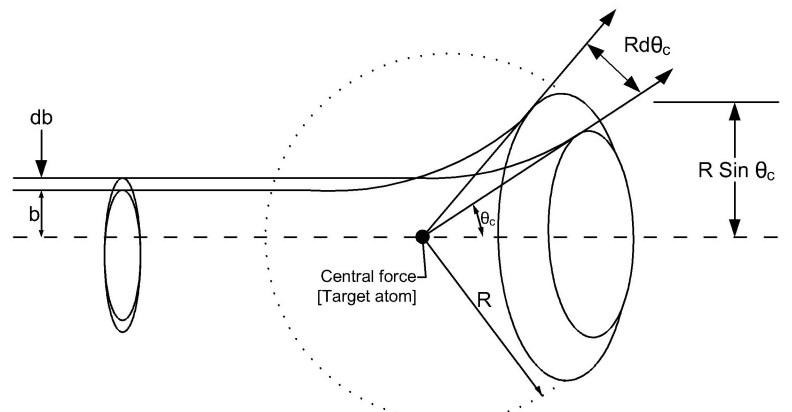

Figure 2.9: Schematic representing the dependence of differential cross-section of solid angle on impact parameter. Similar to scattering angle for ion beam scattering to result in a scattered beam within a window of solid angle, the incident beam must lie within a window of impact parameter. Redrawn from [1]

It can be seen that the solid angle shows similar relationship to the impact parameter as expressed for the scattering angle. The differential cross-section as a function of solid angle is given by [1],

$$
\frac{d \sigma\left(\theta_{c}\right)}{d \Omega}=\left(\frac{Z_{1} Z_{2} e^{2}}{4 E_{c}}\right)^{2} \frac{1}{\sin ^{4}\left(\theta_{c} / 2\right)}
$$

The above expression helps in determining the detection limit in ion beam analysis.

Another useful differential cross-section that is essential in calculation of the damage induced by an ion is the probability that a collision of an ion with energy $E$ onto a target atom results in transferring an energy of $T$ to the recoiling atom. The differential scattering cross-section as a function of recoil energy can be given by [1],

$$
\frac{d \sigma(E)}{d T}=\frac{4 \pi}{T_{M}} \frac{d \sigma\left(\theta_{c}\right)}{d \Omega}
$$

where $T_{M}$ is the maximum energy that can be transmitted to the recoil atom upon impact. This expression allows calculation of differential energy transfer cross-section if the angular differential cross-section is known or if the CM scattering angle and impact parameter is known.

Thus in this section the concept of differential cross-section was introduced and their application in ion beam techniques was presented.

\subsubsection{Ion Stopping}

An energetic ion upon impact onto a solid loses energy by collisions with target atoms and electrons. In the previous few subsections, the focus was on the elastic collisions of the ion 
with the stationary atoms. However, that is just one of the two ways of energy loss. The stopping of ions in matter can be described by,

$$
\frac{d E}{d x}=\frac{d E}{d x}_{n}+\frac{d E}{d x}_{e}
$$

where $n$ denotes nuclear collisions and $e$ denotes electronic collisions.

The equation describes the loss of energy, $d E$, as the ion travels a depth $d x$. This loss is the sum of energy losses due to elastic collisions and inelastic interactions with the electrons of the target atoms. A useful term to denote the stopping process is by defining a stopping cross-section

$$
S \equiv \frac{d E / d x}{N}
$$

where $S$ represents the stopping cross-section per scattering center and $N$ represents the atomic density. The typical units are $\mathrm{eV} . \mathrm{cm}^{3} / \mathrm{nm}$

A proper understanding of the mechanism of energy loss helps in calculating the range, range distribution and lattice disorder in the ion beam impacted material.

\subsubsection{Nuclear stopping}

Consider figure 2.6 again. As an ion traverses length $d x$ in a target of unit area and atomic density $N$ the average energy lost by the ion can be obtained by integrating the differential cross-section for energy transfer, $\frac{d \sigma(E)}{d T}$, for all possible values of $T$ as given in [1],

$$
\langle d E\rangle=N \int_{T_{\min }}^{T_{M}} T \frac{d \sigma(E)}{d T} d T d x
$$

Thus, the nuclear stopping and stopping cross-section is given by,

$$
\begin{gathered}
\left.\frac{d E}{d x}\right|_{n}=N \int_{T_{\min }}^{T_{M}} T \frac{d \sigma(E)}{d T} d T \\
S_{n}(E)=\left.\frac{1}{N} \frac{d E}{d x}\right|_{n}=\int_{T_{\text {min }}}^{T_{M}} T \frac{d \sigma(E)}{d T} d T
\end{gathered}
$$

Ziegler et al, has made several contributions in obtaining accurate expressions for nuclear stopping power [49]. He has derived the nuclear stopping cross-section as a function of reduced energy parameter, $\varepsilon$. The nuclear stopping cross-section in reduced notation is [49],

$$
S_{n}(\varepsilon)=\frac{\varepsilon}{a_{U}^{2}} \int_{0}^{\infty} \sin ^{2}\left(\frac{\theta_{c}}{2}\right) d\left(b^{2}\right)
$$


where the $\theta_{c}$ is $\mathrm{CM}$ scattering angle which depends on the interatomic potential, ion energy and impact parameter. $a_{U}$ is the universal screening length.

The nuclear stopping cross-section thus obtained were fit with numerical methods and the fit was described by two expressions each applicable at different epsilon ranges. For $\varepsilon \leqslant 30$

$$
S_{n}(\varepsilon)=\frac{0.5 \ln (1+1.1383 \varepsilon)}{\left(\varepsilon+0.01321 \varepsilon^{0.21226}+0.19593 \varepsilon^{0.5}\right)}
$$

and for $\varepsilon>30$,

$$
S_{n}(\varepsilon)=\frac{\ln (\varepsilon)}{2 \varepsilon}
$$

For practical calculations, the ZBL universal nuclear stopping for an ion with energy $E_{0}$ in the laboratory frame of reference is

$$
S_{n}\left(E_{0}\right)=\frac{8.462 * 10^{-15} Z_{1} Z_{2} M_{1} S_{n}(\varepsilon)}{\left(M_{1}+M_{2}\right)\left(Z_{1}^{0.23}+Z_{2}^{0.23}\right)} \frac{e V \mathrm{~cm}^{2}}{\text { atom }}
$$

The above equation is the expression used for determining the stopping power in ion beam applications using simulation codes and software.

\subsubsection{Electronic stopping}

Apart from collisions with the stationary atoms in a target, an ion loses energy by inelastic interactions with the electrons of the target atoms. The electrons of target atoms gets excited which can also lead to secondary electron emission from the material. The energy transferred in this process is directly dependent on the charge state of the ions. The charge state or ionization of the ion is dependent on the velocity with which it travels. Bohr suggested that energetic ions will lose electrons whose orbital velocity is less than the ion velocity. The effective charge of the ion is given by [50],

$$
\frac{Z^{*}}{Z}=\left(\frac{v_{1}}{v_{0} Z_{1}^{2 / 3}}\right)
$$

where $Z$ is the total number of electrons surrounding the nucleus at ground state, $Z^{*}$ is the charge on the ion, $v$ is the velocity of the ion, $v_{0}$ is the Bohr velocity i.e., $v_{0}=2.2 \times 10^{6} \mathrm{~m} / \mathrm{s}$, $v_{1}$ is the velocity of the ion and $Z_{1}$ is the atomic number of the ion.

There are two possible states for an energetic ion, $v<v_{0} Z_{1}^{\frac{2}{3}}$ which implies that $\frac{Z^{*}}{Z}<1$ and that the ion is not fully stripped. This denotes the low energy regime. 
The other state is, $v>v_{0} Z_{1}^{\frac{2}{3}}$ which implies that $\frac{Z^{*}}{Z} \cong 1$ and that the ion is fully stripped to a bare nucleus. This is the high energy regime.

Experimentally, the ionization of heavy ion follows more closely the following form,

$$
\frac{Z^{*}}{Z}=1-\exp \left[-0.92 v_{1} /\left(v_{0} Z_{1}^{2 / 3}\right)\right]
$$

The electronic energy loss expression varies for the high energy and low energy regime. In the high energy regime, the interaction between the ion and the electron is pure Coulombic interaction without any shielding effect. An electron is considered in the place of a stationary atom and the energy loss per unit length due to electronic stopping can be given by

$$
T=\frac{\Delta p^{2}}{2 m}=\frac{2 Z_{1}^{2} e^{4}}{b^{2} m_{e} v}
$$

where $p$ is the momentum transferred during collision, $m$ is mass of the ion, $m_{e}$ is the mass of the electron and $T$ is the energy transferred to the electron and thus lost by the ion.

The equation can thus be extended in the terms of differential energy transfer crosssection which gives,

$$
-\left.\frac{d E}{d x}\right|_{e}=n_{e} \int_{T_{\min }}^{T_{M}} T \frac{d \sigma(E)}{d T} d T
$$

where $n_{e}$ is the electronic density. This equation can in turn be written as a function of impact parameter as shown in the below equation,

$$
-\left.\frac{d E}{d x}\right|_{e}=n_{e} \int_{b_{\min }}^{b_{\max }} T 2 \pi b d b
$$

where $b_{\max }$ and $b_{\min }$ refers to the maximum and minimum impact parameter.

$$
-\left.\frac{d E}{d x}\right|_{e}=\frac{4 \pi Z_{1}^{2} e^{4} n_{e}}{m_{e} v^{2}} \ln \frac{b_{\max }}{b_{\min }}
$$

where the maximum energy is lost when impact parameter assumes a value given by,

$$
b_{\min }=\frac{Z_{1} e^{2}}{m_{e} v^{2}}
$$

and the minimum energy lost is equivalent to the smallest energy that can be accepted by an electron to excite to an allowed energy state. If $I$ can be taken to express the minimum amount of energy that can be accepted by an electron, then the high energy electronic stopping turns out to be, 


$$
-\left.\frac{d E}{d x}\right|_{e}=\frac{2 \pi Z_{1}^{2} e^{4}}{E} N Z_{2}\left(\frac{M_{1}}{m_{e}}\right) \ln \frac{2 m_{e} v^{2}}{I}
$$

where $n_{e}=N Z_{2}$, with $N$ given by the atomic density of the stopping medium. This expression is referred to as the Bethe's theory of stopping[51, 52].

The average excitation energy $I$, in electron volts for most elements is roughly,

$$
I \cong 10 Z_{2}
$$

In the case of low energy regime the Bethe's theory of stopping breaks down and a different model of energy transfer is required. Several models of electronic stopping has been developed where the stopping cross-section is assigned to be directly proportional to the velocity of the ions $[53,54,55]$. Widely used model to compute the electronic energy loss in this energy regime was first proposed by Frisov [55]. In his model, he proposed that during ion-atom collision the ion upon impact with the atom forms a quasi particle as shown in the figure below.

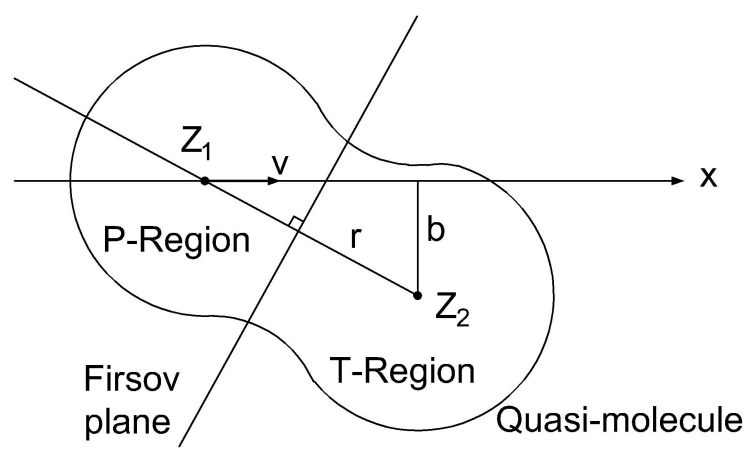

Figure 2.10: Schematic representing quasi particle formed during ion-atom collision with impact parameter $b$ and distance of separation $r$ as proposed by Frisov. Projectile atom with atomic number $Z_{1}$ and velocity $v$ forms the P-region of the quasiparticle and the target atom with atomic number $Z_{2}$ forms the T-region of the quasiparticles. Frisov defined a plane bisecting the line joining the center of the two particles. Electrons passing through this Frisov plane strongly interact with the force field of the corresponding atoms and results in energy loss by electron exchange. Redrawn from [1]

Frisov defined a plane bisecting the line joining the center of the two particles. Electrons passing through this Frisov plane strongly interact with the force field of the corresponding atoms and results in the energy loss as described by,

$$
-S_{e}(E)=2 \pi \int_{0}^{\infty} T_{e} b d b=K_{F} 2 \pi e^{2} a_{0}\left(Z_{1}+Z_{2}\right) \frac{v}{v_{0}}
$$

where $a_{0}$ is the Bohr atomic radius of hydrogen atom, $T_{e}$ is given by 


$$
T_{e}=\frac{0.35\left(Z_{1}+Z_{2}\right)^{5 / 3} h / a_{0}}{\left[1+0.16\left(Z_{1}+Z_{2}\right)^{1 / 3} b / a_{0}\right]^{5}} v
$$

and $K_{F}=1.08$

The Frisov formula fits the experimental data for those collisions where $Z_{1} / Z_{2}$ or $Z_{2} / Z_{1}$ does not exceed 4.

For wide scale application, electronic stopping expression credited to J. Lindhard and M. Scharff is applied [56]. While the derivation of Lindhard-Scharff formula was never published, Sugiyama has shown that the formula can be derived from the Frisov procedure $[57,58,59]$. The point of difference however was attributed to the choice of interatomic potential used by them.

Frisov used the interatomic potential form given by,

$$
V(r)=\frac{\left(Z_{1}+Z_{2}\right) e^{2}}{r^{\prime}} \chi_{T F}\left[1.13\left(Z_{1}+Z_{2}\right)^{1 / 3} r^{\prime} / a_{0}\right]
$$

where $r^{\prime}=r / 2$ is the distance between one of the colliding atoms and the Frisov plane, $a_{0}$ is the Bohr radius and $\chi_{T F}$ is the Thomas-Fermi screening function.

While Lindhard and Scharff provides an interatomic potential given by,

$$
V(r)=\frac{2\left(Z_{1} Z_{2}\right)^{1 / 2} e^{2}}{r} \chi_{T F}\left[1.13\left(Z_{1}^{2 / 3}+Z_{2}^{2 / 3}\right)^{1 / 2} \frac{r}{a_{0}}\right]
$$

The Lindhard-Scharff electronics energy loss equation widely used is given by,

$$
\left.\frac{d E}{d x}\right|_{e}=\xi_{L} 8 \pi e^{2} a_{0} N \frac{Z_{1} Z_{2}}{\left(Z_{1}^{2 / 3}+Z_{2}^{2 / 3}\right)^{3 / 2}}\left(\frac{v}{v_{0}}\right)
$$

where the correction factor $\xi_{L}$ is given by

$$
\xi_{L} \cong Z_{1}^{1 / 6}
$$

The Lindhard-Scharff stopping cross-section can be given as

$$
S_{e}(E)=3.83 \frac{Z_{1}^{7 / 6} Z_{2}}{\left(Z_{1}^{2 / 3}+Z_{2}^{2 / 3}\right)^{3 / 2}}\left(\frac{E}{M_{1}}\right)^{1 / 2}=K_{L} E^{1 / 2}
$$

where $K_{L}$ is given by,

$$
K_{L}=3.83 \frac{Z_{1}^{7 / 6} Z_{2}}{M_{1}^{1 / 2}\left(Z_{1}^{2 / 3}+Z_{2}^{2 / 3}\right)^{3 / 2}}
$$


It has to be noted that so far all the stopping power calculations have been based on the Thomas-Fermi atomic model and does not take into account the electronic shell structure of the atom. This leads to oscillation in the matching of data between the experiment and prediction provided by LS regime. Further the stopping calculated in the high energy regime has an error margin of about $20 \%$.

So far in this section, expressions for nuclear and electronic stopping were developed. It is possible to compare the two stopping power against each other which will show the regions where one dominates the other. Figure 2.11 plots the nuclear and electronic stopping power as function of logarithm of incident ion energy.

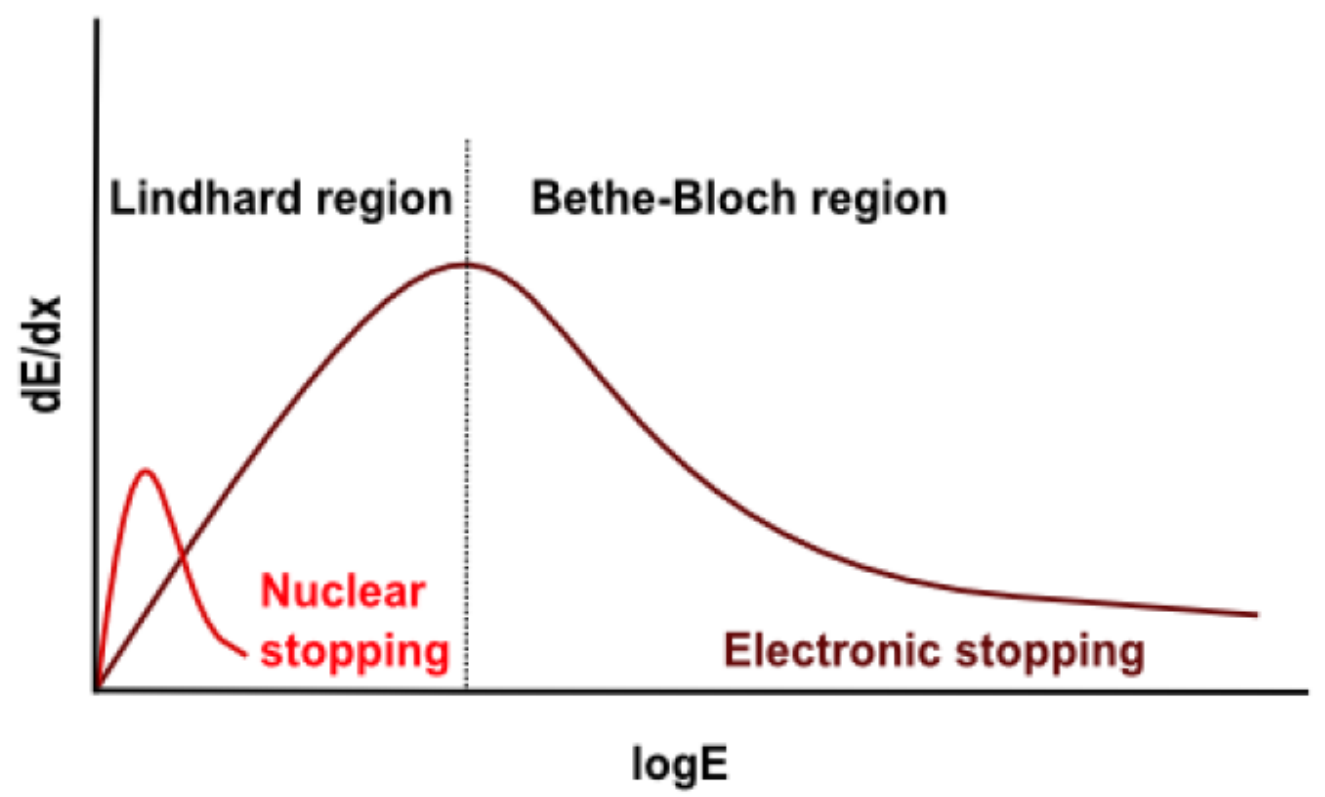

Figure 2.11: Plot of nuclear and electronic stopping with respect to $\log (\mathrm{E})$. It can be observed that the nuclear stopping dominates the energy loss of the projectile at low energy region and the electronic stopping dominates at high energy region. This implies that during ion implantation and ion beam deposition the stopping cross-section that is of importance will be nuclear stopping and in ion beam analysis such as RNRA and RBS electronic stopping will be the key cross-section to be considered [1].

The plot shows that at low energy regimes where ion beam deposition and implantation are carried out the major mode of energy loss is through nuclear collision. It can be further seen that at low energy regime the stopping is proportional to the velocity of the ion as described by the LS equation. As the velocity further increases and the ion approaches the high energy regime nuclear stopping diminishes as $1 / E_{0}$. The electronic stopping in this region is expressed by Bethe's stopping theory. 


\subsubsection{Ion Range and Straggling}

One of the most important parameter in ion beam modification is the range of the incident ion beam. The range determines the extent to which an incident beam can interact with the material. If the interaction distance is limited to near-surface region large changes in the surface properties can be observed. For example, electrical properties are largely dependent on the surface characteristics of the material. On the other hand, low fluence implantation at high energies results in large penetration range of the ion which does not reflect any change in the surface. Before presenting an expression for range and range distribution it is necessary to clarify the different terminologies associated with the concept of ion range.

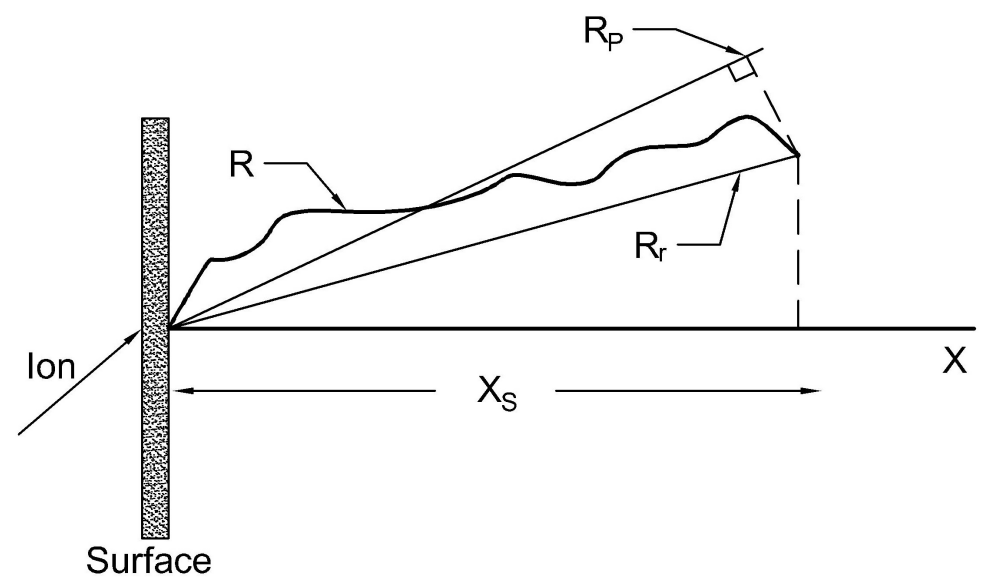

(a) 2-D representation of projected range of implanted ion

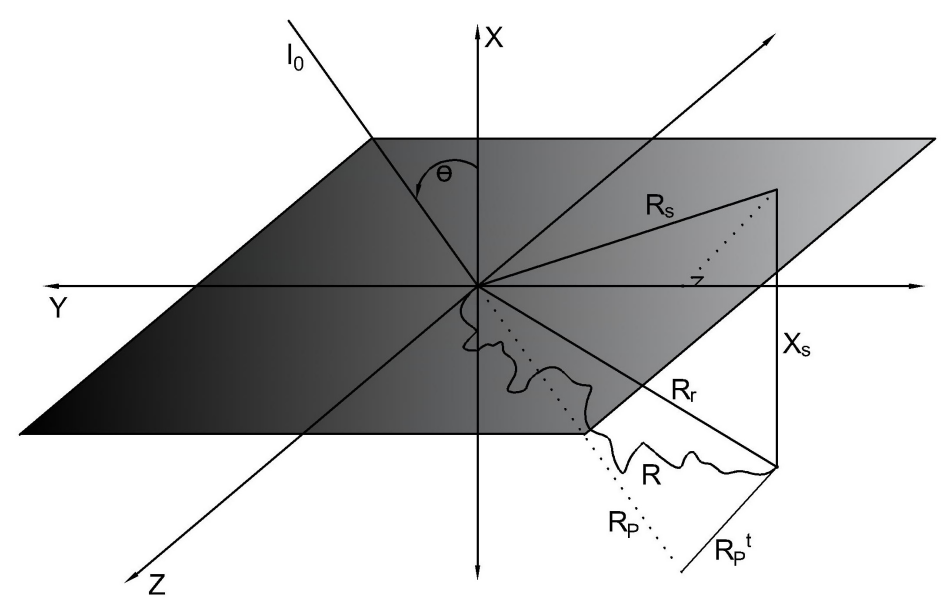

(b) 3-D representation of projected range of implanted ion

Figure 2.12: Schematic representing the different range parameters of an ion implanted into the target surface. Redrawn from [1]

Figure 2.12 shows the range of an implanted ion in the material. Figure 2.12 (a) shows the implantation process in a 2-dimensional form. An incident ion upon entering the target 
undergoes a series of collisions with the electrons and stationary atoms resulting in loss of ion energy. When the ion has lost most of its energy, it can no longer travel further within the material thereby resting at a particular depth from the target surface. The same process is represented schematically in 3-dimensional frame. The final position of the ion in the 3-dimensional frame is given by $\left(x_{s}, y_{s}, z_{s}\right)$. The various terms used to describe the ion travel path are [1],

- Range is defined as the total distance traveled by the ion from its point of entrance at the target surface to its final resting position. The range is directly proportional to its incident energy and inversely proportional to the stopping power.

- Radial range is the vector joining the origin or the point of entrance of the incident ion to its final resting position. If range is the distance covered by the incident ion, radial range refers to the displacement vector of the incident ion. It is given by,

$$
R_{r}=\left(x_{s}^{2}+y_{s}^{2}+z_{s}^{2}\right)^{1 / 2}
$$

- Projected range of an ion refers to the projection of the radial range of the ion onto its incident trajectory vector. Statistical projected range (for a distribution of ions), is the distance measured along the incident ion trajectory from the target surface to the region where the maximum concentration of the implanted ion is found.

- Implant depth is projection of ion range onto a vector normal to the target surface. As the name suggests it refers to the depth at which maximum ions are accumulated into the target. When the incident angle of the ion is normal to the target surface, implant depth and projected range are one and the same.

- Spreading range is the distance between the origin or the point of incidence of the incoming ion at the target surface and the projection of radial range at the target surface. It is given by,

$$
R_{s}=\left(y_{s}^{2}+z_{s}^{2}\right)^{1 / 2}
$$

- Transverse projected range is the vector connecting radial range and the projected range. It is given by,

$$
R_{p}^{t}=\left[\left(x_{s} \sin \theta-y_{s} \cos \theta\right)^{2}+z_{s}^{2}\right]^{1 / 2}
$$

Range definitions are provided in terms of a single ion. However, ion beam processes involve collective effect of a large number of ions. All the ions will not undergo the same number 
of collisions and hence can travel longer or shorter distance compared to other ions. The overall distribution of the ions is thus a statistical process based on the probability of the number and nature of its collisions.

At low fluences and in the absence of channeling the implanted ion depth distribution is roughly Gaussian in nature. The probability function for a Gaussian distribution (normal) [45], is

$$
f(x)=\frac{1}{\sigma(2 \pi)^{1 / 2}} \exp \left[-\frac{1}{2}\left(\frac{x-\mu}{\sigma}\right)^{2}\right]
$$

The depth distribution $N(x)$ of implanted ions, normalized for an ion implantation dose $\phi_{i}$, is given by the expression

$$
N(x)=\frac{\phi_{i}}{\Delta R_{p}(2 \pi)^{1 / 2}} \exp \left[-\frac{1}{2}\left(\frac{x-R_{p}}{\Delta R_{p}}\right)^{2}\right]
$$

where $R_{p}$ denotes the projected range.

In this expression, the probability function is replaced by the depth distribution function $N(x)$, mean $(\mu)$ is replaced by $R_{p}$ and standard deviation $(\sigma)$ is replaced by $\Delta R_{p}$. Assuming all the implanted ions are retained the dose of the distribution can be obtained by integrating the depth distribution for all depths. The peak concentration or the peak atomic density $\left(N_{p}\right)$ of the implanted ion in the distribution is given by,

$$
N\left(R_{p}\right) \equiv N_{p}=\frac{\phi_{i}}{\Delta R_{p}(2 \pi)^{1 / 2}} \cong \frac{0.4 \phi_{i}}{\Delta R_{p}}
$$

The concentration is obtained by,

$$
C_{p}=\frac{N_{p}}{N_{p}+N}
$$

where $N$ is the atomic density.

Now that the distribution is defined by a mathematical description, it is necessary to obtain an expression for (i) Range, (ii) Projected range and (iii) Projected range straggling. The analytical approach to calculate the range quantities was given by J. Lindhard, M. Schraff and H. E. Schiott in 1963 and is commonly referred to as LSS theory. The LSS theory allows computation of range within $20 \%$ error margin. A more accurate calculation is available using the PRAL (Projected range algorithm) developed by J. P. Biersack, U. Littmark and J. F. Ziegler. The simulation program employed in this research work is a refined version of the same.

Range is given by 


$$
R=\int_{E_{0}}^{0} \frac{d E}{d E / d x}=\int_{E_{0}}^{0} \frac{d E}{N S(E)}
$$

where,

$$
\frac{d E}{d x}=\frac{d E}{d x}_{n}+\frac{d E}{d x}_{e}
$$

In the case of ion beam deposition and implantation, the energies involved are in the range of few hundreds of an $\mathrm{eV}$ to $\sim 100 \mathrm{keV}$. At these ion energies the stopping due to electronic contribution is insignificant and the range can be approximated to result purely from the nuclear stopping. Such a range estimate obtained from approximation is given by $[1]$,

$$
R\left(E_{0}\right)=\left(\frac{1-m}{2 m}\right) \frac{\gamma^{m-1}}{N C_{m}} E_{0}^{2 m}
$$

where

$$
\gamma \equiv \frac{4 M_{1} M_{2}}{\left(M_{1}+M_{2}\right)^{2}}=\frac{T_{M}}{E}
$$

and

$$
C_{m}=\frac{\pi}{2} \lambda_{m} a_{T F}^{2}\left(\frac{2 Z_{1} Z_{2} e^{2}}{a_{T F}}\right)^{2 m}\left(\frac{M_{1}}{M_{2}}\right)^{m}
$$

where $\mathrm{m}=1 / 3$ when $\varepsilon<0.2, \mathrm{~m}=1 / 2$ when $0.2<\varepsilon<2$ and $\mathrm{m}=1$ for $\varepsilon>10$ (RBS). The projected range can be calculated from range (Lindhard, 1963)

$$
R_{p} \cong \frac{R}{1+\left(M_{2} / 3 M_{1}\right)}
$$

The calculations so far were based on the assumption that the target is made of a single element. In the case of poly-atomic target, accurate calculations require the use of PRAL code. However an approximate value can be derived by,

$$
R_{p}\left(A_{x} B_{y}\right) \cong N_{\text {alloy }}\left[\frac{\left(R_{p}(A) / N_{A}\right)\left(R_{p}(B) / N_{B}\right)}{\left(y R_{p}(A) / N_{A}\right)+\left(x R_{p}(B) / N_{B}\right)}\right]
$$

A very important parameter accompanying calculation of range and projected range is the calculation of straggling. Ion implantation is a random process and projected range merely denotes the average depth at which maximum concentration of the implanted ions comes to rest. Some ions can undergo lesser collisions and can thus travel greater distance while some can experience greater number of collisions and thus comes to rest closer to 
surface. It is a natural process that cannot be eliminated or even controlled in ion beam processes. It could hence be viewed as a disadvantage of ion beam technology.

However, the implanted ion distribution as mentioned earlier can be described in most cases accurately by a Gaussian distribution. The full width half maximum of this distribution is determined by the straggling of the implanted ions. In the discussed energy regime where nuclear stopping dominates, straggling can be determined to fair level of accuracy based on the mass of the ion and target atom alone. The range straggling was calculated based on Lindhard theory and is given by,

$$
2.5 \Delta R_{p} \cong 1.1 R_{p}\left[\frac{2\left(M_{1} M_{2}\right)^{1 / 2}}{M_{1}+M_{2}}\right]
$$

or

$$
\Delta R_{p} \cong R_{p} / 2.5
$$

However the accuracy of the above expression holds good only when $\varepsilon<3$ and when $M 1>M 2$. This is because when $M 1<M 2$, the ion atom collisions involve large angle scattering and hence would require simulation works such as PRAL to obtain a proper value of the straggling value.

\subsubsection{Atomic displacements and Sputtering}

In the sections so far various phenomena of ion-solid interactions were discussed. It was shown that an energetic ion loses energy by electronic excitations and nuclear collisions leading to energy loss and eventual stopping of the ion within the solid. An expression was arrived to determine the range of the ion and the straggling experienced by it in its path. An important consideration of such interactions is the impact delivered by the ion onto the material. The nuclear stopping especially causes significant damage within the base material by forming vacancies and interstitials. This effect is especially important when the base materials are crystalline in nature. In the case of ion implantation each crystalline material has an implantation threshold beyond which the material becomes completely disordered. This threshold is referred to as amorphization threshold and Si for example has a typical amorphization threshold in the range of few $10^{14}$ atoms.cm ${ }^{-2}$.

An ion upon collision with a target atom can result in displacement of the target atom from its lattice position. Consider the energy transferred by an ion onto a target atom $(T)$ during a collision process. If $T$ is greater than a threshold value $E_{d}$, the collision will result in a displacement of the target atom and results in formation of a Frenkel pair. This 
threshold value $E_{d}$ is referred to as the displacement energy. The displacement energy is directly proportional to the binding energy or the sublimation enthalpy. On the other hand, if $T<E_{d}$ then the collision just results in vibration of the target atom in its lattice position which spreads to other atoms as phonons. If the incident energy is much greater than the displacement energy then atoms displaced from their lattice position can cause further displacements in the target lattice. These atoms are referred to as primary knock-on atoms (PKA). The PKA can lead to secondary knock-ons, tertiary knock-ons etc., leading to a cascade of atomic collisions, usually termed as collision cascade.

The number of displacements and total displacement damage resulting from an incident ion with an energy $E_{0}$ is given by [1],

$$
N_{p}\left(E_{0}\right)=\int_{0}^{E_{0}} \sigma_{d}\left(E^{\prime}\right) \frac{d E^{\prime}}{S\left(E^{\prime}\right)}
$$

where $E^{\prime}$ is ion energy as function of distance traveled by the ion. Another crucial information that is particularly important for this work is the spatial distribution of damage energy within the base matrix. This expression is given by [1],

$$
F_{D}(x)=\frac{E_{0}}{2 m R}(1-x / R)^{\frac{1}{2 m}-1}
$$

Sputtering refers to the process of ejection or removal of atoms from the target upon impact by an energetic ion beam. Sputtering occurs when the atoms present on the surface of the target are displaced outward due to either direct collision with the incident ion or by a chain of energy transfer from the incident ions through knock-on atoms. For an atom present at the surface, the displacement energy is determined by its surface binding energy. A useful parameter for evaluating the loss due to sputtering is the sputter yield. Sputter yield denotes the number of atoms sputtered from the target surface due to impact by a single ion [1].

$$
Y \cong 4.2 \alpha S_{n}\left(E_{0}\right) / U_{0}=4.2 \alpha(d E / d x)_{n}\left(N U_{0}\right)^{-1}
$$

where $Y$ is the sputter yield, $S_{n}$ is the nuclear energy loss, $\alpha$ is a function based on the ratio of the masses of target atom to the incident ion, $N$ is the atomic density in $(n m)^{-3}$ and $U_{0}$ is the surface binding energy. Sputtering has two important effects in this research work. The first effect is that, during implantation presence of significant sputtering can result in reduction in the thickness of the target. This leads to a shift in the implanted ion distribution towards the surface. In such a scenario, the depth at which the maximum implanted ion concentration is measured becomes closer to the surface with implantation fluence (when 
the implanted ion is heavier than the target). Prolonged implantation can further lead to non-Gaussian distribution. The second effect of sputtering is the achievement of steady-state condition. This is achieved when the sputter yield of the implanted ion reaches 1 . In such a scenario, no further increase in the implanted ion concentration can be achieved. Any further implantation will cause equal number of implanted ions to be sputtered out.

\subsubsection{Simulations}

The concepts discussed so far cover the basic interaction between an energetic ion and the target matrix. In many instances analytical solutions were sought to describe the slowing down and scattering of ion in matter. Computer simulations can similarly be used to describe the same. Two such types of simulations generally exist: (a) Monte-Carlo simulations (b) Molecular dynamics simulations. Monte-Carlo simulations applies binary collision approximation to evaluate the interactions. Molecular dynamics approach on the other hand considers the interaction as a many-body problem of Newtonian mechanics for many interacting particles. In this work, the simulation methods utilized are based on Monte-Carlo approach.

Few underlying assumptions involved in general in an MC approach are,

1. The target is considered to be amorphous

2. Binary collision approximation is applied

3. The nuclear and electronic energy losses are considered to be independent of each other

4. Nuclear stopping is based on the interatomic potential given by Lindhard [56]

5. Electronic stopping at low velocities are based on the formalism of Lindhard and Scharff and at high velocities are given by the Bethe formula

6. The contribution of each species of a poly-atomic target is proportional to their number density.

The simulation in this work are based on computer code known as Dynamic Transport and Range of Ion in Matter (D-TRIM) [60]. A major advantage of using it is that compared to other simulation codes, this software considers the dynamic changes in composition during the simulation work. The use of the code to compute the ion range, damage and elemental distribution and sputtering effects are discussed in detail in the results chapter. 


\subsection{Magnetic Properties}

Magnetic properties of transition metal doped DLC has not been explored much and is an important contribution made by this work. Few basic concepts regarding the magnetic properties of materials are briefly discussed below.

\subsubsection{Origin of magnetism}

Magnetism arises from moment of charged particles. In materials, electrons are the mobile carriers of charge. Hence the distribution and interaction of electrons with other electrons dominate the magnetic behavior of the material. The atomic nucleus also possesses a magnetic moment but the contribution from electron is about $10^{3}$ times stronger than the nuclear moment.

Electrons can contribute towards a magnetic moment in two different ways. One contribution arises from their orbital angular momentum and the other from their intrinsic angular momentum also referred to as "Spin". Figure 2.13 attempts to represent both these contributions in a classical picture. However, it is to be noted that the quantum mechanical nature of electron does not have a classical analogue.

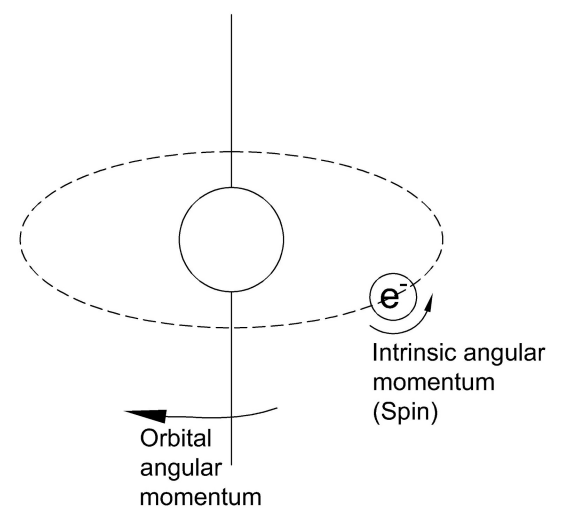

Figure 2.13: Schematic representing electron contribution to magnetic moment. Two contributions as observed in the figure are the magnetic moment due to orbital angular momentum of the electron and magnetic moment arising from the intrinsic angular momentum of the electron. Redrawn from [2]

Figure 2.13 shows the motion of electron in the first Bohr orbit around the nucleus. The magnetic moment $(\mu)$ arising out of its orbital motion can be calculated to be

$$
\mu=(\text { area of loop }) \times(\text { current })
$$

Which leads to 


$$
\mu(\text { orbit })=\frac{e h}{4 \pi m_{e}}
$$

The figure also denotes the spin of the electrons. The magnetic moment due to electron spin calculated from theory and experiment turns out to be

$$
\mu(\text { spin })=\frac{e h}{4 \pi m_{e}}=\frac{\left(1.60 \times 10^{-19} \mathrm{C}\right)\left(6.62 \times 10^{-34} \mathrm{Js}\right)}{4 \pi\left(9.11 \times 10^{-31} \mathrm{~kg}\right)}=9.27 \times 10^{-24} \mathrm{~A} . \mathrm{m}^{2}
$$

It can be seen that the magnetic moment due to spin and the orbital motion (1st Bohr orbit) are both equal. The value of this magnetic moment is referred to as the Bohr magneton.

$$
\mu_{\mathrm{B}}=\text { Bohr magneton }=e h / 4 \pi m=9.27 \times 10^{-24} \mathrm{~A} \cdot \mathrm{m}^{2}
$$

\subsubsection{Classification of magnetic materials}

When a magnetic field is applied to a free electron it tends to align its magnetic moment in the direction of the applied field to reduce its overall energy. However, when the electrons are distributed in a material they arrange themselves based on their interaction with the atomic nucleus and the surrounding environment. In those materials where all the electron shells are fully occupied each electron is paired with another electron of opposite spin and hence there is no net magnetic moment exhibited by the material. When a magnetic field is applied on such materials they develop a net magnetic moment opposing the applied field and are hence termed diamagnetic.

Those materials which contain an unpaired electron in the outer most shells in presence of external magnetic fields develop a net magnetic moment in the direction of the applied field. In the absence of an external field, the magnetic moment from the unpaired electrons are randomly oriented and hence do not contribute towards a net magnetic moment. Such materials are termed paramagnetic.

There are three further types of magnetic behavior observed in condensed stated of matter. They are ferromagnetism, ferrimagnetism and anti-ferromagnetism. They arise due to interactions of electrons with their neighbors.

Consider two atoms brought very close to each other. The electrons of these atoms have to assume a different distribution due to the effects of space localization. The interaction between the electrons of the two atoms are governed by the Coulombic potential and application of Pauli's exclusion principle. This forces the electrons to assume either a triplet state where the electrons share the same spin but a different spatial coordinate or a singlet 
state where they share the same spatial coordinate but their spins are aligned anti-parallel to each other. This interaction can be represented by [2],

$$
E_{e x}=-2 J_{e x} S_{i} S_{j}
$$

where $E_{e x}$ is the exchange energy, $J_{e x}$ is referred to as exchange integral and $S_{i}$ and $S_{j}$ represents the angular moments of two neighboring atoms.

If the exchange integral is positive, the system attains the lowest energy state when the neighboring electrons are aligned parallel to each other. Such materials possess a spontaneous net magnetic moment and are referred to as ferromagnetic materials.

When the exchange integral is negative, it denotes that the electrons are oriented antiparallel to each other. If the moments of the electrons are equal in value, the material does not exhibit a net magnetic moment and are hence termed anti-ferromagnetic. If the moments are unequal, then they exhibit a net magnetic moment equal to the magnitude of their differences. Such materials are termed to be ferrimagnetic.

It has to be noted that while ferromagnetic and ferrimagentic material possess spontaneous magnetization, generally a small external field is required to achieve a maximum net magnetic moment referred to as saturated magnetic moment. As per the theory of exchange interactions this external field is unnecessary. Weiss explained this apparently contradictory findings by projecting the domain picture of magnetic materials. He proposed that a ferromagnetic material is divided in many domains (small regions within the material), where each domain is spontaneously magnetized in a particular axis of magnetization. The boundaries of each of these domains are referred to as domain walls. Since a ferromagnet is composed of many such domains oriented in random directions, the net magnetization is zero. When an external magnetic field is applied, all it does is flip these domains in the direction of the applied field. When two neighboring domains point towards the same axis of magnetization the domain wall separating them vanishes and they become a single domain. A material magnetized to its maximum value is pictured to be composed of a single domain.

\subsubsection{Temperature and field dependence}

A useful quantity to describe the magnetic order in these material is magnetization. Magnetization is defined as the net magnetic moment present in a unit volume of the material. The magnetization is dependent on the applied magnetic field as given below,.

$$
M=\chi H
$$

where, $M$ is the magnetization, $H$ is the magnetic field and $\chi$ refers to the susceptibility 
of the material.

Ferromagnetic and ferrimagnetic materials exhibit spontaneous magnetization, (i.e.) application of even small amounts of magnetic field can led to large magnetization. This is attributed to their exchange interactions, which act as an internal molecular field. This effect can be described efficiently by plotting magnetic susceptibility as a function of magnetic field. Figure 2.14 describes this relation for different magnetic materials.

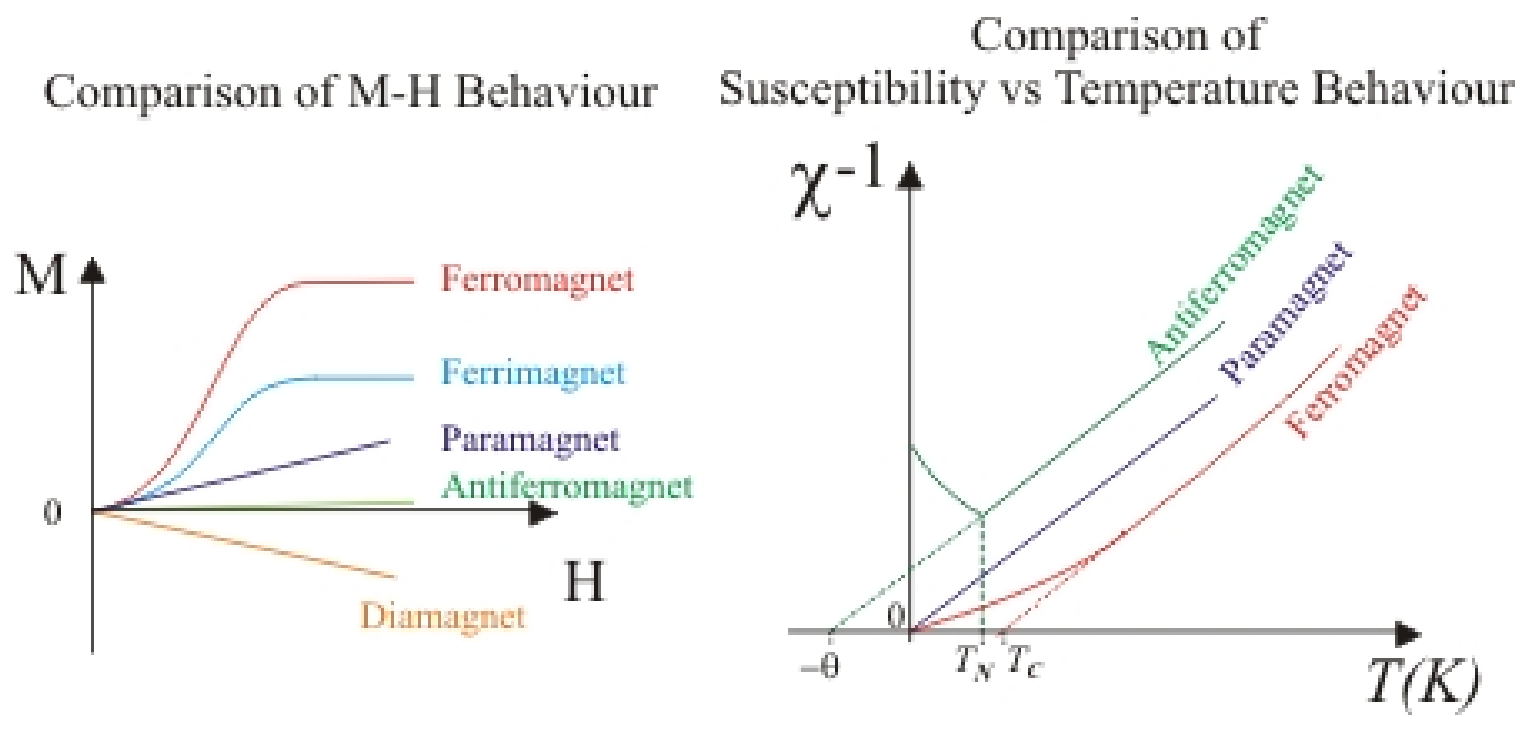

Figure 2.14: Magnetization and magnetic susceptibility of different magnetic materials with varying field and temperature. Redrawn from [2]

As mentioned before, external magnetic field has a tendency to increase the magnetic ordering within a material. However at all temperatures above the absolute zero, thermal energy plays a role opposite to that of the applied magnetic field. In other words, thermal energy causes randomization of the electron spins within the material.

In the case of diamagnetic materials, there is no spin contribution to the magnetic behavior of the material. Hence the magnetic susceptibility of these materials do not vary with temperature. Paramagnetic materials on the other hand shows continuous drop in magnetic susceptibility with increase in temperatures. This relation is described by Curie's law [61].

$$
\chi=\frac{C}{T}
$$

where, $C$ is the Curie constant and $T$ is the temperature

In ferromagnetic materials, there is a positive shift in the magnetic susceptibility vs temperature curve due to the presence of molecular field/ exchange interactions. Their relation is described by Curie-Weiss law, 


$$
\chi=\frac{C}{T-\theta}
$$

where $\theta$ is known as the Curie-Weiss temperature.

Another relation that is worth explaining is the dependence of saturated magnetization of ferromagnetic materials with temperature. When all the electronic spins of a material are aligned in the direction of the applied field, the magnetization of the material is said to have saturated. The saturated magnetic moment at absolute zero is denoted by $m_{s}(0)$. At all temperatures above $0 \mathrm{~K}$, the randomization effect prevents the saturated moment to achieve $m_{s}(0)$. The variation of the saturated magnetic moment with temperature can be described by Bloch function, as shown below [22],

$$
m_{s}(T)=m_{s}(0)\left[1-B \times T^{\beta}\right]
$$

where $m_{s}$ represents the saturated moment, $B$ is a constant inversely proportional to spin stiffness coefficient and $\beta=3 / 2$ for Bloch function. The Bloch function accounts for the randomization effect induced on the magnetic order by thermal energy in the form of quantized spin waves / magnons. The Bloch equation describes the magnon dispersion as a function of temperature.

\subsubsection{Magnetic nanoparticles}

Confinement of materials to nanoscale dimension leads to several interesting properties. In the case of magnetic properties, ferromagnetic nanoparticles exhibit a behavior known as superparamagnetism. As the name suggests, the material exhibits paramagnetic behavior but with a large magnetic susceptibility.

A ferromagnet generally has an easy axis of magnetization and attains lowest energy state when ordered in its easy axis. Changing its magnetization direction from its easy axis requires it to pass through an energy barrier. This property is commonly referred to as magnetocrystaline anisotropy. Ferromagnet exhibits coercivity and remanence due to the presence of this property. The reduction in energy of the material by aligning in its easy direction is referred to as the anisotropy energy of the material. The anisotropy energy is proportional to the volume of the material and is given by [2],

$$
M A E=k V
$$

where $\mathrm{V}$ is the volume of the material and $\mathrm{k}$ is the anisotropy coefficient which is unique for each material. Superparamagnetism is observed when the size of the magnetic nanoparticles 
go below the size of the magnetic domains. In such a scenario, the anisotropy energy of the nanoparticles are comparable to their thermal energy i.e., $k V$ is comparable to $k_{B} T$. In this case, the randomization effect caused by the thermal energy distorts the material from its magnetically ordered state. The entire nanoparticle acts as a single spin with a super moment. Such nanoparticles assumes the behavior of a paramagnetic material when $k_{B} T>$ $k V$ and a ferromagnetic material when $k_{B} T<k V$. The temperature of transition is referred to as blocking temperature and can be measured experimentally by Zero Field Cool - Field Cool (ZFC-FC) measurements. In this measurement, the sample containing nanoparticles are measured against increasing temperature starting from both their magnetically ordered state and a random state. The point at which the ZFC-FC curve splits or joins indicate the blocking temperature of the material. At temperature below the blocking temperature the sample exhibits irreversible behavior which denotes the presence of coercivity. The utility of ZFC-FC measurement along with their application in this work is discussed in chapter 7 .

\subsection{Conclusion}

This chapter in total provides an introduction to ion-solid interactions and magnetic properties of materials. The various concepts involved in the interaction of energetic ions with matter is discussed in light of the different ion beam processes used in this work. In particular, the concepts of cross-section, ion stopping, range, damage and sputtering are discussed in detail. A section was devoted to touch upon the basic concepts and classification of magnetic material. A detailed use of the concepts mentioned above can be found in the results chapter (chapter 4 - 7). 


\section{Chapter 3}

\section{Methods}

This chapter covers the experimental setup and limits of various techniques used in this research. The objective is to study the structural, magnetic and electrical properties of Co implanted DLC films. For this purpose different characterization techniques with varying capabilities are used. Each characterization technique offers a unique perspective on the material and using a set of complimentary techniques a complete understanding of the material can be achieved.

The various steps in this research work are,

1. Growth of DLC films by ion beam deposition

2. Co implantation into as-deposited DLC films

3. Simulation of Co implantation profiles in DLC

4. HR-RBS analysis of the Co distribution in the implanted films

5. TEM imaging of the implanted regions in the DLC films

6. Performance of resonant nuclear reaction analysis on implanted and as-deposited DLC to measure hydrogen depth profile

7. Raman measurement on the as-deposited and implanted DLC films

8. Measurement of magnetic behavior as a function of magnetic field and temperature using a magnetic property measurement system

9. Measurement of electrical conductivity as a function of measurement temperature using physical property measurement system

Each of the above mentioned techniques are presented as individual sections of this chapter. 


\subsection{Ion implantation}

Ion implantation is a technique widely used in the electronic industry. The main application of ion implantation is in the introduction of dopants into the semiconducting wafer used for IC fabrication. Ion implantation was first recorded in literature in the form of four patents filed in the year 1950 and 1954 by three originators Ohl, Moyer and Shockley [62, 63, 64, 65]. They have discussed the use of ion implantation to generate defects and substitute atoms in the base matrix that can lead to doping. From then on several technological developments and industrial and research requirements led to wide spread use of ion implantation technology. Ion implantation plays a crucial part in this research work. While DLC can be doped by different means, ion implantation can induce specific changes and unique properties in DLC due to the energetic interactions involved in this process. Thus the method of doping plays a crucial role in the properties exhibited by the DLC film. It is therefore necessary to examine the technique in detail. The ion-solid interactions that happen during the process are explained in detail in section 2.1. In this section, the experimental setup of the implanter is discussed.

As per the methodology, the first step of this work is to deposit diamond-like carbon films by ion beam deposition. However, it would be beneficial to start the experimental chapter with the explanation of the ion implanter. Understanding the various components making up the implanter enables better understanding of the experimental setup making up the ion beam deposition system.

The overall function of an ion implanter is to bombard a target surface with energetic ions. The various components making up the implanter allows generation of ions, selecting the energy and mass of the generated ions, focusing the ions onto the target and providing control over the number of ions bombarded per second over the target area (current density). 


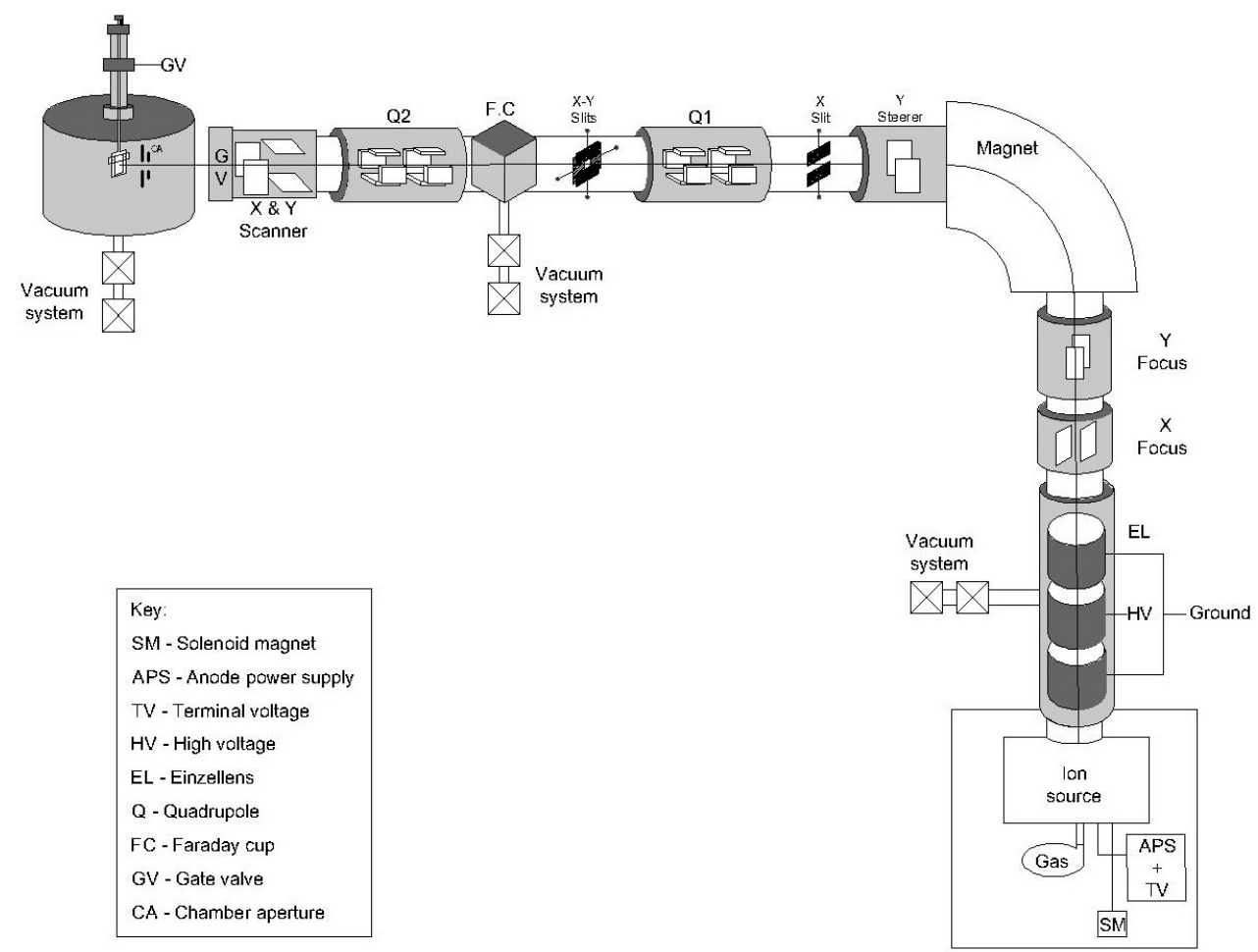

(a) Schematic of industrial ion implanter

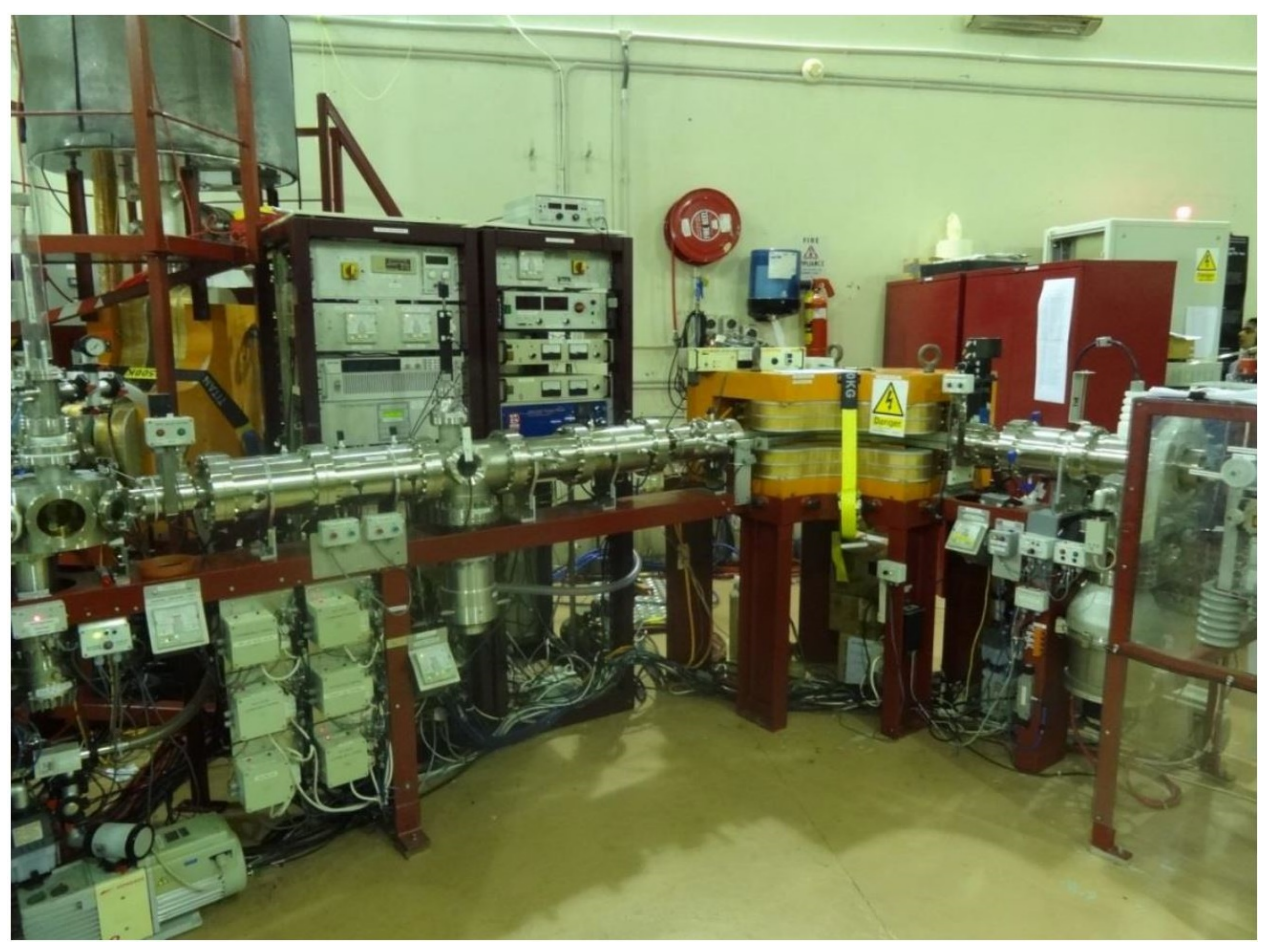

(b) Industrial ion implanter

Figure 3.1: Industrial implanter at GNS Science

A schematic of the ion implanter used at GNS Science [66] along with its picture is shown 
in figure 3.1. The key components making up this ion implanter are:

1. An ion source which generates the ions required for implantation.

2. Beam transport components and columns which transports and focuses the ion beam onto the target.

3. An double focusing electromagnet to segregate the different constituents of the ion beam and select a particular mass and energy.

4. A sample chamber which houses the sample target and provides easy access for loading and unloading samples.

5. Vacuum systems to evacuate the ion source, beam line, electromagnet and sample chamber.

The working of these different sections of the ion implanter will be discussed in the following steps,

\subsubsection{Ion source}

The ion implanter at GNS science uses a Penning ion source to produce the necessary ion beams [67]. A schematic of Penning ion source is shown in figure in 3.2. The ion source contains a hollow anode in between two cathodes as shown in the figure 3.2. This entire setup is enclosed within a solenoid magnet. The anode is connected to a high voltage feed-through via a spark plug that can withstand upto $3000 \mathrm{~V}$ at $100 \mathrm{~mA}$ current. The anode is typically operated at a potential of about $2 \mathrm{kV}$. The solenoid is connected to a power supply that provides upto $3 \mathrm{~A}$ at $15 \mathrm{~V}$. Under normal working conditions the solenoid current typically ranges from $1-2.3 \mathrm{~A}$ (at $5-11.5 \mathrm{~V}$ and $5 \mathrm{ohm}$ resistance).

The working principle of a Penning ion source is based on Penning discharge which produces a plasma by application of a cross product of electric and magnetic fields. Cosmic rays creates the first set of primary electrons in the ion source. These electrons upon hitting other neutral atoms generate an avalanche of electrons. These electrons further get accelerated towards the anode due to application of high voltage. A precursor gas is let into the system via a gas feed in the outlet located at the back cathode. The aim is to generate a plasma by ionizing the precursor gas by the energetic electrons. This process requires a large number of collisions between the primary electrons and the gas molecules. This is achieved by applying a magnetic field through the solenoid. The magnetic field forces the electrons to travel in a helical path thereby increasing the probability of collisions between electrons and the 
gas molecules. The electrons are expected to have undergone approximately, 200-300 turns during their travel from the cathode to anode. The ionization leads to a plasma enclosed within the anode and cathode geometry. The magnetic and electric field also confines the electrons and ions inside the ion source. The positive ions are extracted from the ion source through a hole in the front cathode of the ion source (shown in figure 3.2).

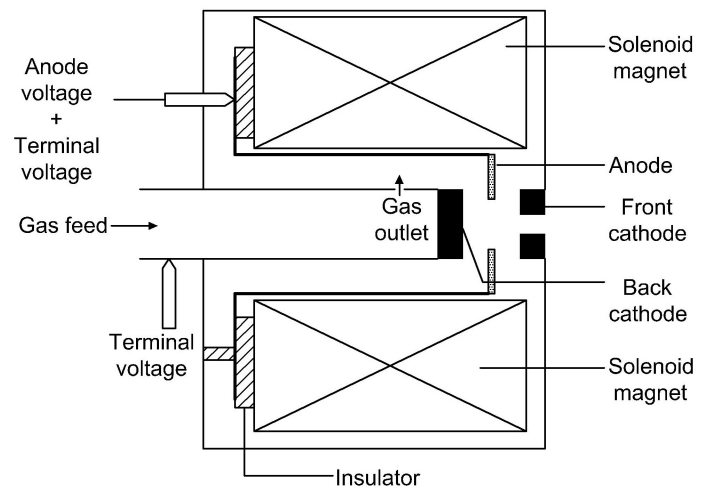

Figure 3.2: Schematic representing Penning Ion source

In order to implant gas ions, the respective gas is sent as precursor gas to the ion source. In this case the anode current generated is in the order of $1-4 \mathrm{~mA}$. The gas pressure is in the range of $10^{-6} \mathrm{hPa}$ at the Einzellens. On the other hand if the aim is to implant the target with ions from a solid material, the respective solid is loaded as a cathode material within the ion source. The precursor gas in this case is generally a noble gas. The plasma generated from the noble gas is used to sputter the cathodes to produce the required ions. Neon is typically used a the precursor gas in the implanter as it has the optimum balance between its ionization cross-section and sputtering capacity. The anode current in the case of sputtered ions ranges from $10-40 \mathrm{~mA}$ and the gas pressure is in the range of $10^{-5}$ to $10^{-4} \mathrm{hPa}$. Use of noble gas ensures minimal chemical interaction between the gas ions and the cathodes. It is also to be noted that the functioning of the ion source requires solid conductive cathodes. If the cathode is an insulator, conducting metals are mixed with the cathode material to increase their conductivity. Dedicated ion sources are available at GNS science for each of the above cases.

\subsubsection{Ion extraction}

The next important step after the production of ions is the extraction of the ions from the ion source. As can be seen in figure 3.1, the entire ion source is supplied with a terminal voltage which acts as the acceleration voltage for the produced ion beam. The industrial ion implanter used at GNS Science for this project can provide a terminal voltage upto 40 
$\mathrm{kV}$ and can thus generate singly charged ions with energy upto $40 \mathrm{keV}$. Since the entire ion source is supplied with the terminal voltage, the plasma and the ions generated within the ion source remain unaffected by the terminal voltage inside the ion source. The ions exiting the ion source however have the following parameter influencing their extraction,

- The anode voltage which drives the positive ions to exit the ion source

- The acceleration voltage (terminal voltage) which effects the ions immediately upon exit due to the large potential gradient between the ion source and rest of the system

- The gap between the ion source exit and entrance to the Einzellens

- The space charge effect which can limit the extracted ion current
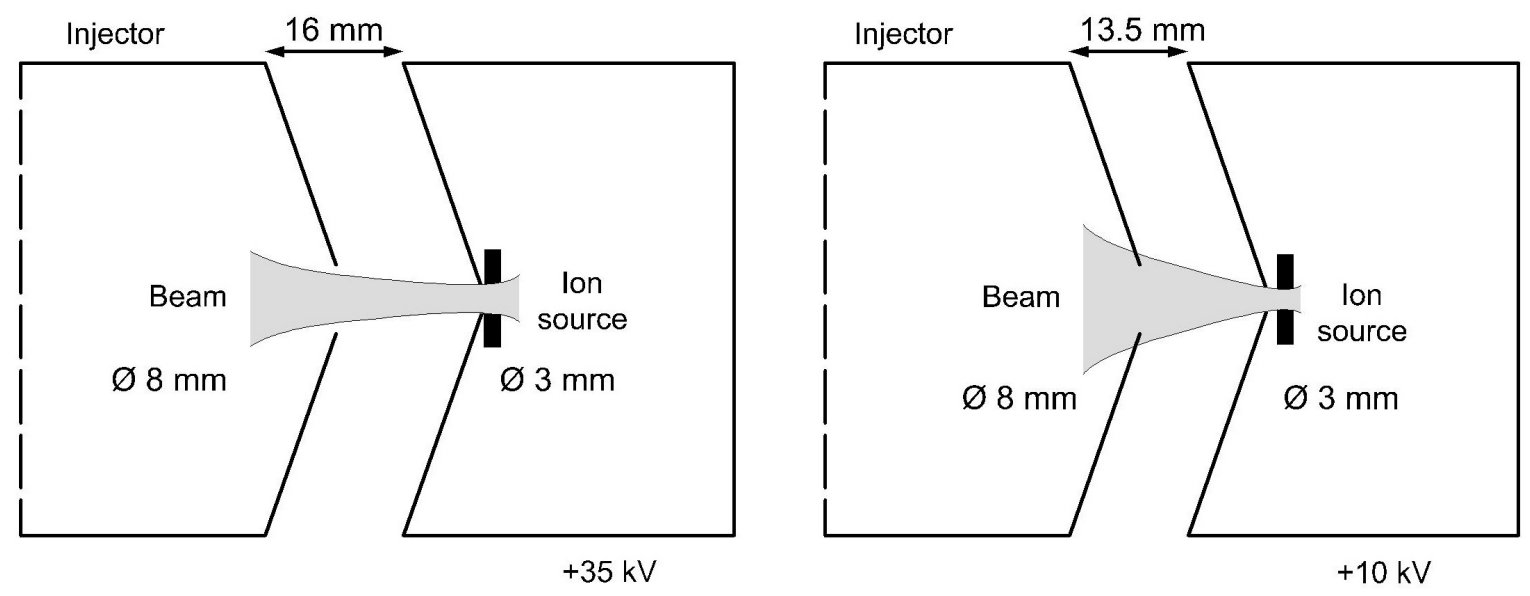

Figure 3.3: Schematic representing ion extraction from ion source to the injector system starting with the Einzellens. The separation distance between them has to increased with increasing terminal voltage to prevent arcing and improve the transmission efficiency of the ion beam generated from the ion source.

The effect of anode voltage and terminal voltage in the extraction process is straight forward. Increase in both the parameters generally leads to higher ion currents. Spacecharge affect refers to the repulsion between the positive ions confined in the small space. Limiting effects are significant only for currents greater than several $\mathrm{mA}$. The effect of the gap between the nose cone of the ion source and the Einzellens is shown in figure 3.3. It can be seen that the ions exiting the ion source spread in a radial fashion exiting at all possible angles. At low acceleration voltage, this gap is reduced to a minimum to ensure effective capture of all ions exiting the ions source. However, at the energies utilized for Co implantation $(30 \mathrm{keV})$ the gap is maximized to prevent arcing between the ion source and Einzellens. The acceleration voltage is strong enough to direct the ions towards the Einzellens nose cone. 


\subsubsection{Ion beam focusing}

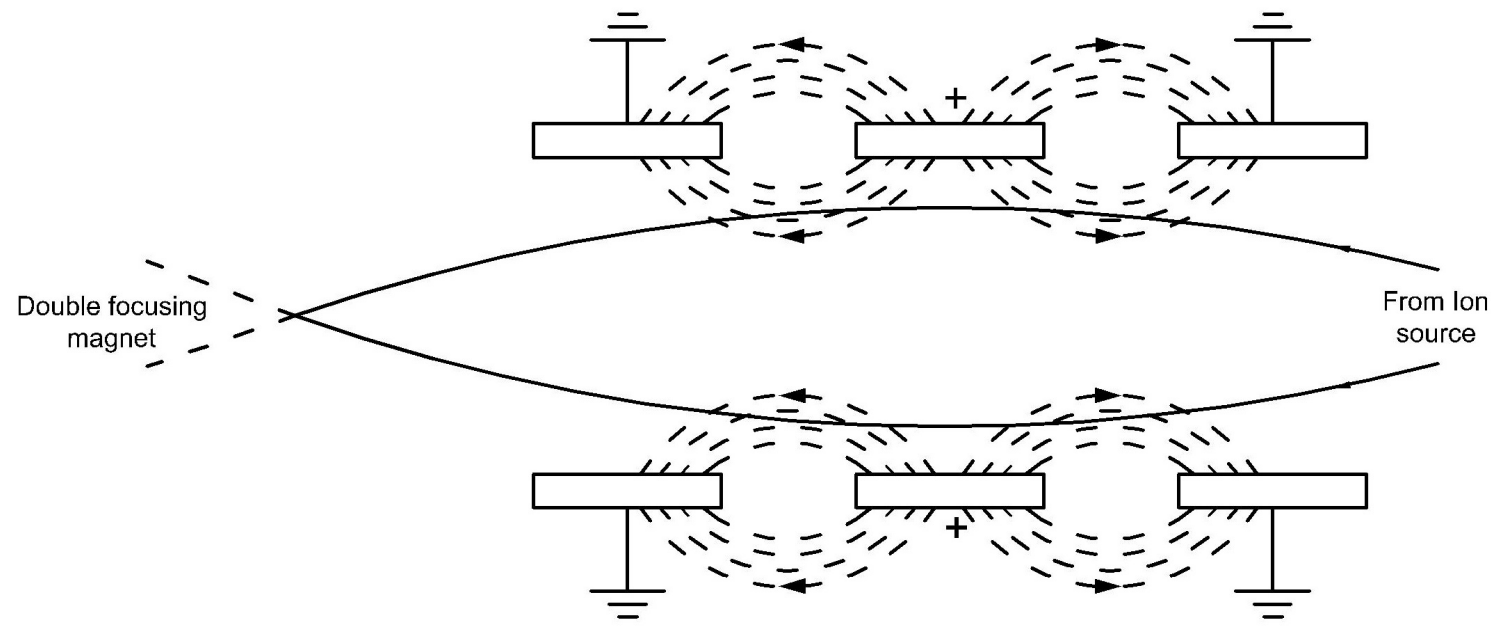

Figure 3.4: Schematic representing 2D cross-section of ion path in the Einzellens. The ions entering the Einzellens are usually focused to the center of the double focusing electromagnet used for mass-separation in the ion implanter.

The Einzellens refers to a set of three metallic hollow cylinders placed on the path of the ion beam as shown in figure 3.1 [68]. Of the three cylinders, the middle component is connected to a high voltage potential and the other two components are grounded to earth. The electric field lines acting between the middle cylinder and the outer cylinders provides a lensing effect to the ion beam extracted from the ion source as shown in figure 3.4. The Einzellens does not alter the velocity and thus the energy of the ion beam. This is due to the symmetry of the field lines between the middle cylinder and the outer cylinders. As seen before the ion beam proceeds radially outward during extraction. The function of the Einzellens is to focus the ion beam towards the center of beam line to produce a stable ion current. The Einzellens at GNS Science is designed in such a way that it focuses the ion beam into the center of the electromagnet. The focus point can be adjusted by varying the Einzellens voltage. 


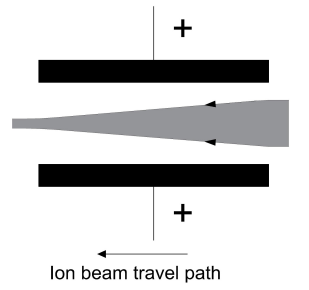

(a) 2D Cross section of ion path $\mathrm{Y}$ focus

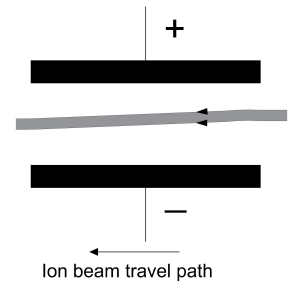

(b) 2D Cross section of ion path in Y steerer

Figure 3.5: Schematic representing ion focusing and steering in the ion implanter

The ion beam then passes through a set of $\mathrm{X}$ and $\mathrm{Y}$ lenses which further focuses the beam towards the center. Figure 3.5 (a) shows the function of a $\mathrm{Y}$ focus. The component is made of set of two metallic plates positioned at the top and bottom of the beam line. These plates are connected to a power supply. Since the beam is composed of positive charges, a positive bias is applied to both the plates to produce a focusing effect as schematically shown in the figure. The implanter also uses a set of steerers for steering the ion beam. The steerers are similar to the lenses except that in this case one of the plates assume a positive bias and the other a negative bias. They alter the path of the ion beam in the beam line as shown in figure 3.5 (b).

\subsubsection{Mass separation}

The ion beam focused by the Einzellens and X and $\mathrm{Y}$ lenses are mass separated by passing them through an electromagnet. The implanter employs a 1.4 Tesla $90^{\circ}$ double focusing electromagnet controlled by a power supply providing a maximum current of $160 \mathrm{~A}$ at $30 \mathrm{~V}$. The electromagnet applies a magnetic field in the direction perpendicular to the path of the ion beam. Application of magnetic field induces a Lorentz force which in turn results as a centripetal force on the ion beam. Their relation is shown in equation $3.1-3.4$.

$$
\begin{gathered}
F_{L}=q(v \times B) \\
\frac{m v^{2}}{r}=q(v \times B)
\end{gathered}
$$




$$
\begin{aligned}
& B=\frac{m v}{q R} \\
& r=\frac{m v}{q B}
\end{aligned}
$$

where $F_{L}$ denotes the Lorentz force, $q$ is the charge of the ion, $v$ its velocity, $B$ the applied magnetic field, $m$ is the mass of the ion and $r$ is the radius of the ion under the magnetic field.

In this work, $\mathrm{Co}^{+}$is the ion of interest. Co has a mass of 59 a.m.u. It has a $1+$ charge and is accelerated by $30 \mathrm{kV}$. The velocity of the ion calculated from its energy is $3.13 \times 10^{5}$ $\mathrm{m} / \mathrm{s}$. The electromagnet has a bending radius of $400 \mathrm{~mm}$, however in the above calculations a bending radius of $420 \mathrm{~mm}$ is utilized to correct for the misalignment between the magnet and the beam line. Inputting these values suggest the applied magnetic field to be 4564 Gauss (refer table 3.1). Figure 3.6 further shows a schematic of Co mass separation through the electromagnet. The mass separation can further be enhanced by use of slits after the electromagnet. For the current application, the slits are closed to allow an ion beam of 10 mm diameter.

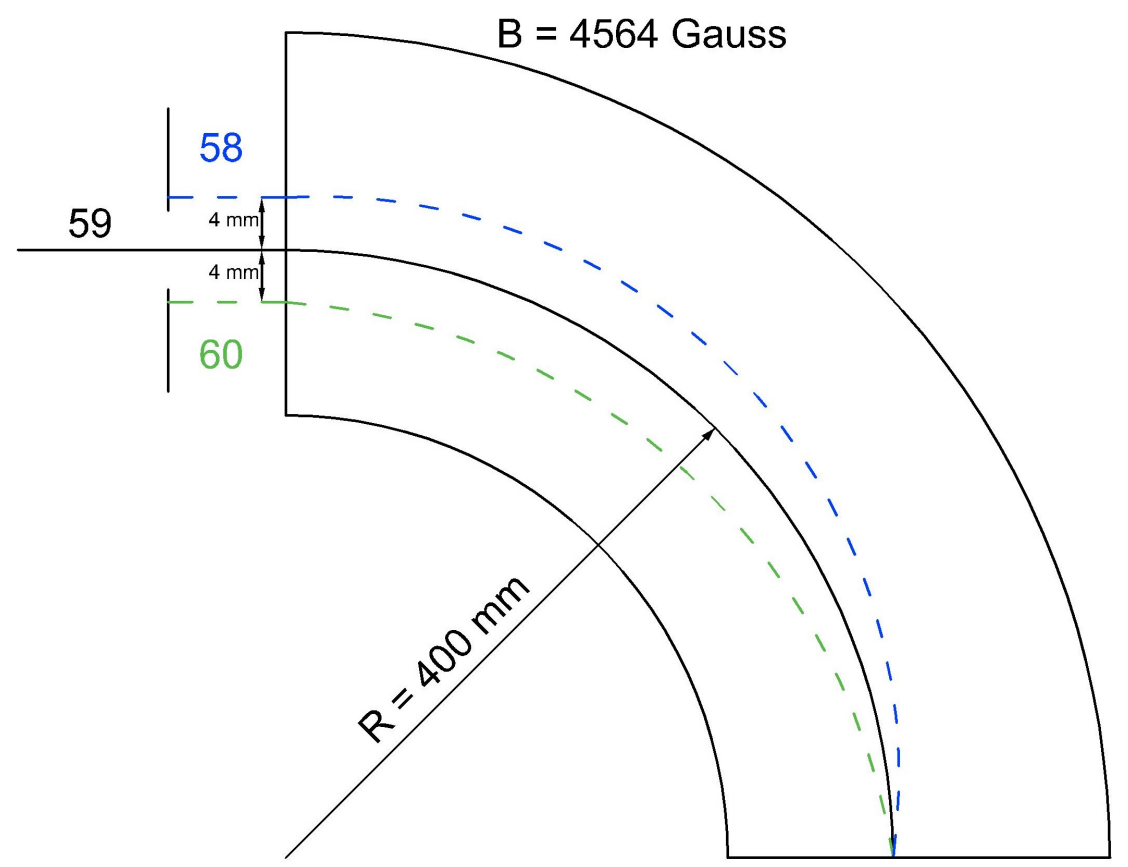

Figure 3.6: Schematic representing mass separation in Industrial Implanter at GNS Science 
Table 3.1: Mass separation by electromagnet in Industrial implanter at GNS Science

\begin{tabular}{|c|c|c|c|}
\hline Mass (m) & Energy (E) & Magnetic field (B) @ fixed $\mathrm{r}=420 \mathrm{~mm}$ & Radius @ fixed B = 4564 G \\
\hline a.m.u & $\mathrm{keV}$ & Gauss & $\mathrm{mm}$ \\
\hline \hline 58 & 30 & 4526 & 416 \\
\hline 59 & 30 & 4564 & 420 \\
\hline 60 & 30 & 4603 & 424 \\
\hline
\end{tabular}

\subsubsection{Quadrupole focusing}

This ion beam then passes through a Y steerer to ensure the beam remains in the center of the beam line. This function is important before the beam passes through the quadrupoles. Quadrupole refers to a set of four metallic plates with hyperbolical surfaces placed on the path of the ion beam to correct for its shape and focus it towards the sample target. The ion implanter at GNS uses two duplet quadrupole systems. For practical reasons, the plates were machined to have circular metallic face instead of a hyperbola.

In general the first quadrupole (Q1) functions to correct the beam path and passes it onto the second quadrupole(Q2) which focuses it towards the target. However, effective focusing and maximization of ion beam current requires an advanced setup of the quadrupole system where Q1 along with beam path correction must also focus the beam in such a way that it creates a beam cross-over in between of the two quadrupoles. This mirrors the initial cross-over observed in the extraction of ions from the source to the Einzellens. Maximum current were recorded when the quadrupoles were setup in this manner.

Another notable feature is that use of quadrupole require only one tenth of the potential required for operating the Einzellens. The voltage required reduces with increasing order of focusing (slits to Einzellens to Quadrupoles). It needs to be mentioned however that quadrupole can only be used for symmetrical input ion beams and has a low acceptance angle. It is for this very same reason an Einzellens was used for ion extraction. As no control over beam shape is possible upon immediate exit from the ion source Einzellens which has a large acceptance angle was employed to ensure maximum beam extraction. 


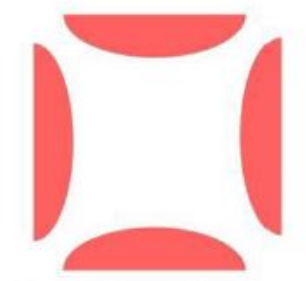

Single lens Front view

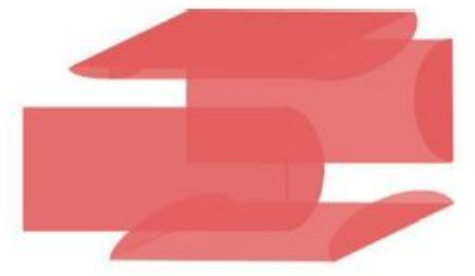

Single lens side view
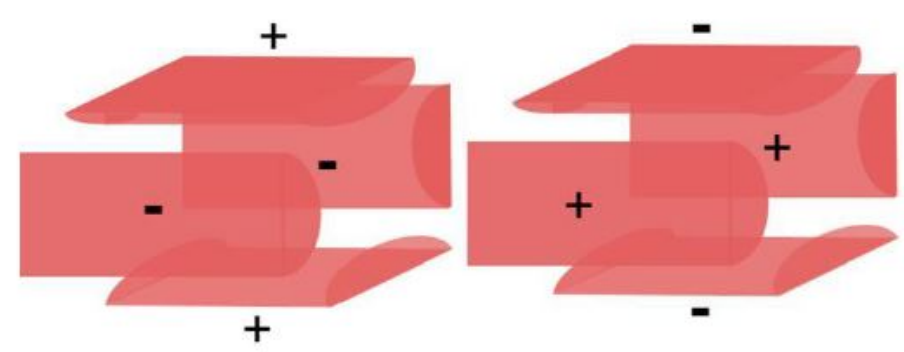

Figure 3.7: Duplet quadrupole system at the Industrial Ion implanter

\subsubsection{Scanning}

The last component the ion beam passes before entering the sample chamber is the $\mathrm{X}$ and $\mathrm{Y}$ scanners. The X and Y scanners have a setup similar to X and Y steerers. The key difference however is that instead of a DC voltage an AC voltage is applied onto the metallic plates which continuously steer the beam in positive and negative $\mathrm{X}$ and $\mathrm{Y}$ directions. The scanner is connected to a $2000 \mathrm{~V}$ power supply and usually the $\mathrm{Y}$ scanner is operated at $80 \mathrm{~Hz}$ and $\mathrm{X}$ scanner at $30 \mathrm{~Hz}$. Scanning also reduces the ion beam current incident on the sample as it steers the entire beam in all the four directions. Generally, half of the unscanned current is lost in the scanning process as it is essential to obtain uniform implantation.

\subsubsection{Sample Chamber}

The ion beam thus passed on from Q2 is focused onto the sample target loaded in the sample holder present within the sample chamber. The sample chamber is a cylindrical stainless steel container placed with a circular aperture on the path of the ion beam. The top lid of the chamber is fit with a sample exchange column to allow easy loading and unloading of the samples. The chamber aperture and the sample holder are connected to a charge integrator which accurately measures the current incident on them as ion beam. Measuring the ion 
beam current incident on the sample holder (where the targets are placed) allows calculation of the ion fluence and measuring the current incident on the chamber aperture preceding the sample holder helps in centering the ion beam and estimating the appropriate scanning required for a particular ion beam setup. The sample chamber also houses an electron gun which is connected to a $400 \mathrm{~V}$ power supply. The electron gun is used to continuously spray the sample with electrons. This ensures the charge neutrality of the samples. Without an electron gun, continuous bombardment of positive ion beam on the samples especially on DLC can cause sample charging which can decelerate or even deflect the ion beam.

Three turbo pumps along with a roughing pump are employed to ensure high vacuum conditions during ion implantation. A high vacuum is a per-requisite for transport of ion beam from the source to the sample chamber. Einzellens, Faraday cup and sample chamber are each connected to a turbo pump. Differential pumping along with liquid nitrogen trap at the sample chamber establishes a base vacuum of $10^{-8} \mathrm{hPa}$ vacuum at the sample chamber. These are the primary components making up the ion implanter system at GNS Science.

\subsection{Direct Ion Beam Deposition}

Ion beam deposition of DLC films were carried out by two techniques: Direction ion beam deposition (DIBD) and mass-selective ion beam deposition (MSIBD). DIBD as the name suggests refers to a process of thin film deposition in which a carbon or carbon rich ion beam is bombarded onto a substrate to produce DLC films. A schematic of the DIBD system built at GNS Science exclusively for deposition DLC films is shown in figure 3.8 [3]. 


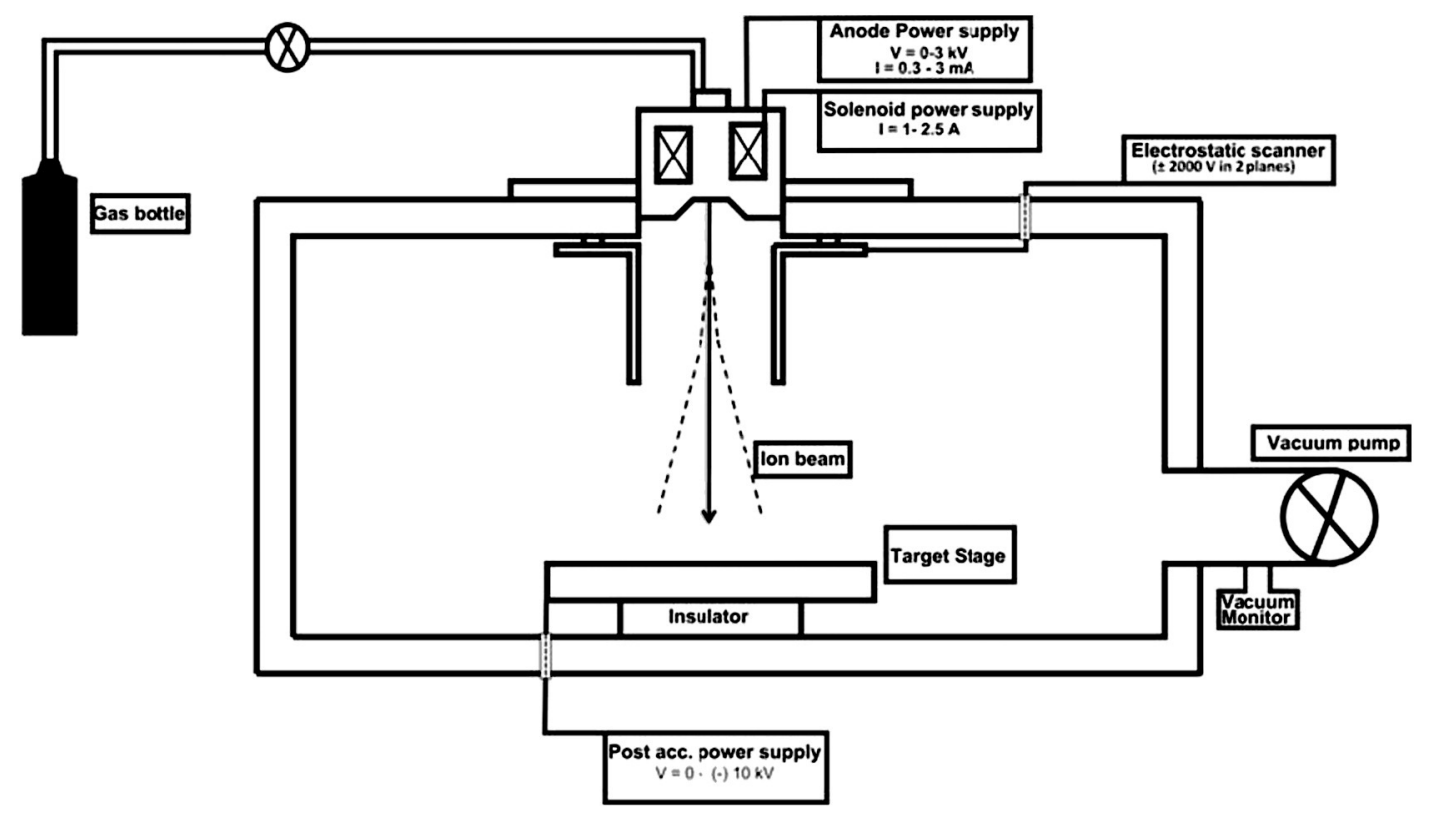

Figure 3.8: Direct ion beam deposition system at GNS Science. Source [3]

The setup consists of the following components

1. Penning ion source: To produce the required ion beam

2. Electrostatic scanner: To ensure uniformity and increase surface coverage

3. Sample stage: For placement of sample and acceleration of the ion beam

4. Sample chamber: For containing the whole apparatus at vacuum

5. Vacuum system: To pump out the sample chamber and ion source so as to ensure high vacuum within the system

The Penning ion source functioning is explained in detail in the previous section. The ions extracted from the Penning ions source are directed towards the sample stage. DIBD system does not contain an extraction system employing an acceleration voltage. Use of acceleration voltage is essential to maintain a stable beam in an ion implanter however is not essential in ion beam deposition system due to its simplified setup. On the path of the ion beam are the electrostatic scanners that are basically four metallic plates connected to an external power supply. Each plate is placed perpendicular to each other covering the four possible directions of the horizontal plane. Application of alternating voltage to these plates can result in scanning of the ion beam onto the sample stage. The sample stage is a cylindrical metallic stage placed on path of the ion beam. The stage is large enough to capture the complete ion beam and the substrate for DLC deposition is placed on it. The metallic stage 
is connected to a negative power supply. By applying a negative bias to the sample stage, the incoming ion beam can be accelerated towards the substrate. Silicon was chosen as the substrate for DLC deposition as it is diamagnetic and does not contain oxygen or nitrogen impurities.

One drawback present in the current system is that it lacks any means to reduce sample charging effect produced during ion beam incidence. The ions are positively charged and the stage is applied a negative bias voltage. If the substrate is highly resistive it can build up a layer of positive charges on the top surface preventing any further ion bombardment. Hence, the method restricts the use of high resistive substrate for DLC deposition. Since DLC will act as the substrate for Co implantation the research work does not require a specific substrate. All DIBD samples were prepared using low resistive Si substrate which implies that they are heavily doped. The implication of this restriction is that films prepared by the DIBD system cannot be used in magnetization studies as heavily doped Si substrate gives off noisy background signals during the magnetic measurements.

\subsection{Mass-Selective Ion Beam Deposition}

DIBD deposition of DLC is a relatively simple process that works without mass separation of the ion beam. The important difference in mass-selective ion beam deposition is the use of an electromagnet to separate the incoming ion beam into its various constituents [36]. In this technique a particular ion species from the different constituents are selected for depositions. The key impact of mass selection lies in the control of ion energy during the deposition process. Selection of ion species also allows control over the C:H ratio in the DLC film. The ability to select the ion energy and ion species has significant impact on the overall structure of the DLC film. The setup used for MSIBD is shown in figure 3.9. 


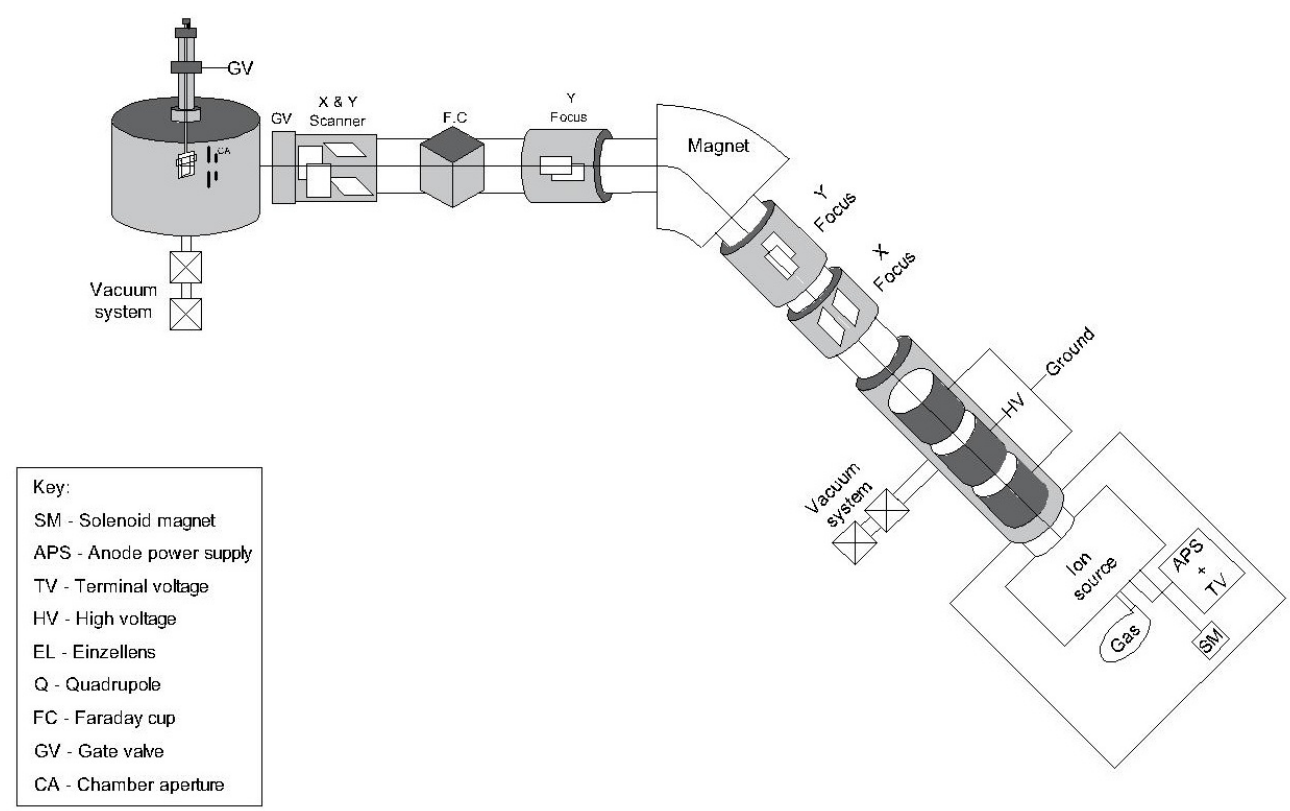

Figure 3.9: Schematic representing mass selective ion beam deposition system at GNS Science

Figure 3.9 shows the schematic of the mass-selective ion beam deposition used to deposit DLC films. The schematic is very much similar to the ion implanter. In fact the principle difference between the two setup is the reduced energy of operation and lower mass resolution due to use of a $45^{\circ}$ bending magnet in place of a $90^{\circ}$ bending magnet. The system consists of a Penning ion source for ion generation followed by an Einzellens for focusing the extracted ion beam onto the $45^{\circ}$ electromagnet which splits the ion beam based on its charge and mass. The mass separated ion beam is then focused onto the substrate held by a target holder which is housed in the sample chamber. High vacuum is achieved by using a combination of turbo-molecular pump and rotary pump. The system is evacuated by pumping system connected at three different regions: (i) at the Einzellens (ii) X and Y steerer (iii) Sample chamber.

A Penning gas ion source similar to the one used at DIBD is used in this system. The ion source is evacuated to a base pressure of $8 \times 10^{-7} \mathrm{hPa}$ before introducing butane as precursor gas to an operating pressure of $2 \times 10^{-5} \mathrm{hPa}$. The anode voltage is maintained between 1.5 to $2 \mathrm{kV}$. The anode current is usually limited to $2-4 \mathrm{~mA}$ and solenoid current is limited to $1.5 \mathrm{~A}$ to create a cross over between the electric and magnetic field. This leads to ionization of the precursor gas to create a carbon rich plasma. Carbon ions are extracted from the front cathode of the ion source by the anode voltage. It can be seen in the schematic that the whole ion source is placed at a terminal voltage supplied by an external power supply. Typically, a terminal voltage of $3 \mathrm{kV}$ is supplied to provide a total acceleration voltage of $5 \mathrm{kV}$ (terminal + anode voltage). The extracted ion beam then passes onto the Einzellens which focuses 
the ion beam towards the center of the electromagnet. The Einzellens is usually applied a voltage very close to the acceleration voltage $\sim 4.5-4.8 \mathrm{kV}$.

The ions focused onto the electromagnet experience a transverse magnetic field which causes bending of the ion beam due to Lorentz force. DLC films used for Co implantation are deposited using $\mathrm{C}_{3} \mathrm{H}_{6}^{+}$ion species, which has a mass of 44 a.m.u and possess $5 \mathrm{keV}$ energy. A magnetic field of 2560 Gauss was applied to achieve the desired mass selection. The selected ion species are then passed onto X and Y steerers which directs the ion beam onto the target stage. Steerers are metallic plates which deflects the ion beam based on application of electric potential. The ion beam before bombarding the substrate is scanned in the $\mathrm{X}$ and $\mathrm{Y}$ direction by a set of electrostatic scanners.

Unlike DIBD system the target holder does not hold any bias and the sample chamber contains an electron gun similar to the ion implanter setup which neutralizes the substrate and prevents it from charging up. The deposited ions are detected and measured by a charge integrator which enables control over the implantation fluence. Accurate measurement of the implantation fluence is possible with this setup. An implantation fluence of $5 \times 10^{17}$ molecules.cm ${ }^{-2}$ gives rise to DLC films with thickness of about $\sim 100 \mathrm{~nm}$.

\subsection{High resolution Rutherford backscattering}

Rutherford backscattering spectrometry is used in this work to measure the depth profile of implanted ions in DLC matrix [47]. The basic principle of this technique is covered in section 2.1. In this section, the instrument and the experimental setup used during the measurement are presented.

In Rutherford backscattering, the composition and distribution of different elements within the target matrix is probed by use of a highly energetic ion beam. The collision process between the energetic ions with the stationary target atom results in backscattering of a small fraction of the incident beam. The scattering angle and the energy of the ions reveals information about the mass and position (depth) of the target atom involved in the scattering process.

Hence for this measurement, the key requirements are

i) An accelerator setup to provide a stable high energy ion beam

ii) A detector placed at a specific scattering angle to measure the energy of the scattered ion

Apart from the above requirements, all RBS setup will use beam optics to focus and filter the incident ion beam, a sample chamber and holder sometimes fit with a goniometer to position the target with respect to the incident ion beam and vacuum systems to evacuate 
the whole setup to satisfactory pressure levels.

The HR-RBS measurements were carried out at the Center for Ion Beam Applications (CIBA) at the National University of Singapore [4]. At CIBA, there is a single-ended 3.5 MV Singletron TM accelerator from High Voltage Engineering Europa (HVEE) attached to three beam lines as shown below in figure $3.10[69,5]$. The accelerator has a beam stability of $\pm 20 \mathrm{eV}$ and a brightness of $20 \mathrm{pA} /\left(\mu \mathrm{m}^{2} \mathrm{mrad}^{2}\right) \mathrm{MeV}$. High stability of the beam energy ensures minimum contribution from energy fluctuations towards broadening the system energy resolution. In depth details about the accelerator can be found from [69].

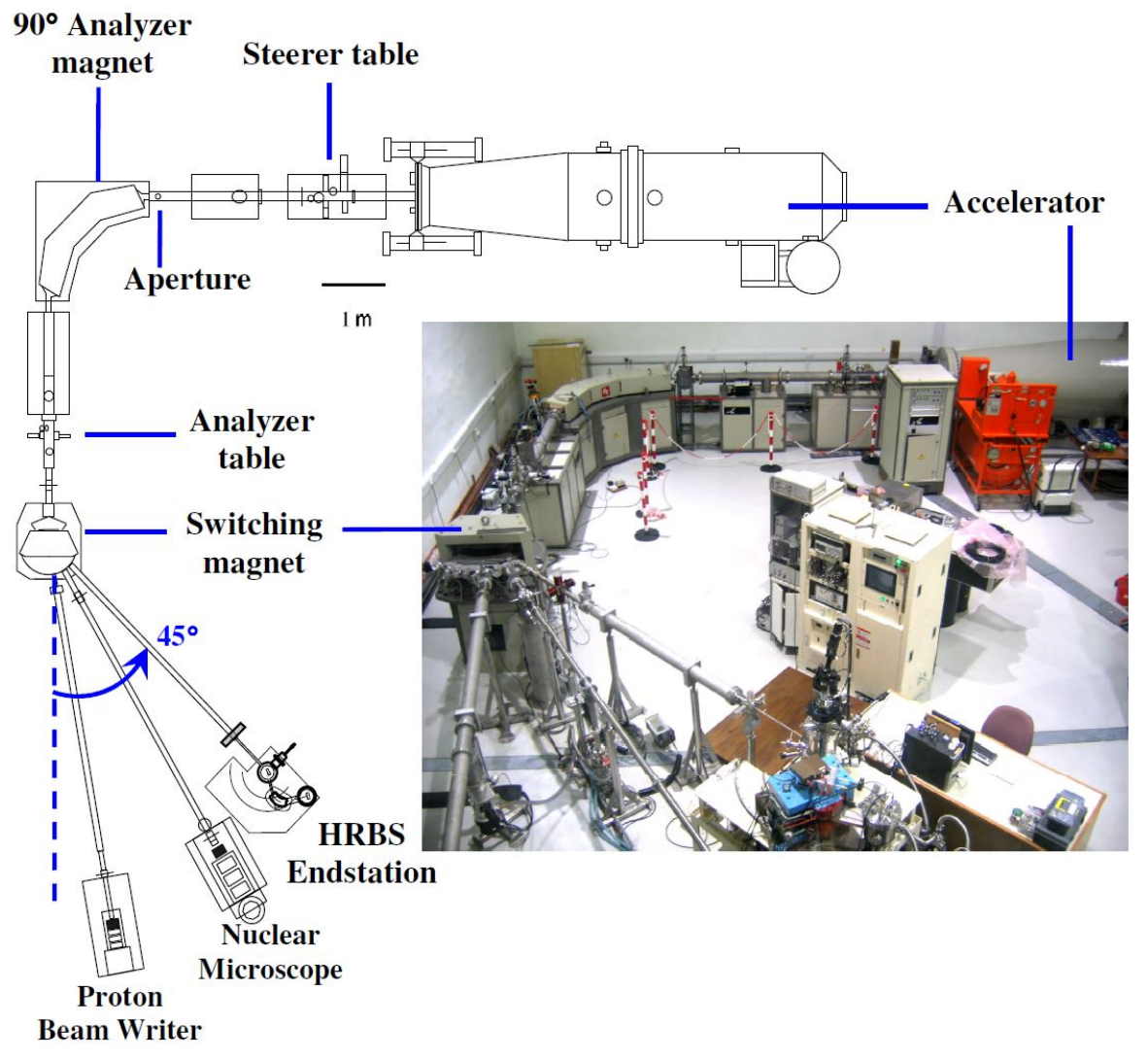

Figure 3.10: Layout of the accelerator and beamlines at CIBA, NUS [4]

The beam from the accelerator is passed through a set of steerers and slits coupled with a beam profile monitor and a Faraday cup to ensure proper steering and focusing of the incident ion beam onto the switching magnet which in turn steers the beam to one of the beam lines. The HR-RBS end station is connected to the $45^{\circ}$ beam line from the accelerator.

The HR-RBS end station consists of 
1. Main chamber with a load lock chamber

2. Ultra high vacuum system (pumps, valves and interlocks)

3. 5-axis goniometer

4. Control cabinet

5. Spectrometer magnet and Multi channel place - focal plane detector chamber (HR-RBS detector system)

The scattering chamber is maintained under a constant pressure of $5 \times 10^{-6} \mathrm{hPa}$ by use of turbomolecular pumps. The load-lock chamber works similar to the setup at the GNS ion implanter allowing easy loading and unloading of the sample to the scattering chamber. The sample holder is connected to a computer controlled goniometer which allows translations in all three dimensions and rotation about the vertical and horizontal axis. The ion beam scattered from the target then passes onto the magnetic spectrometer via a $2 \mathrm{~mm}$ collimeter. 


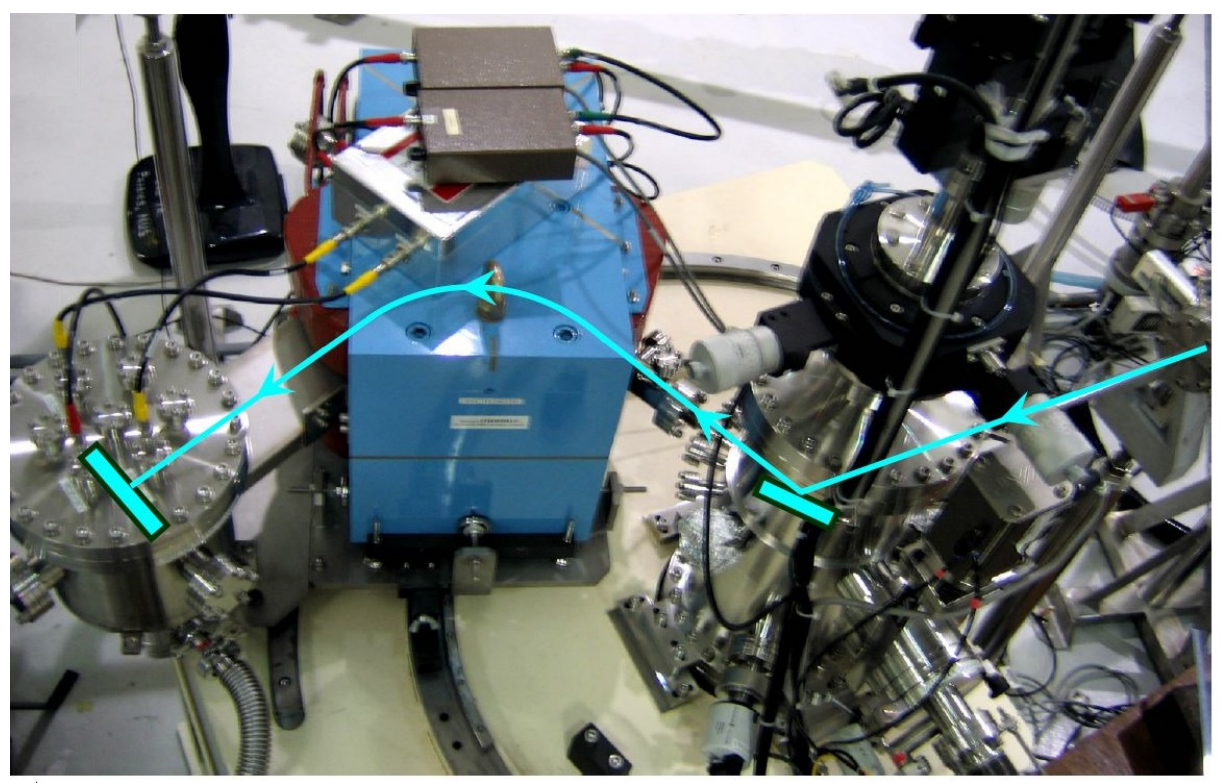

(a) Actual HR-RBS detector system. The path of the ion beam scattered from the sample and passing onto the detector through the electromagnet is highlighted in the picture.

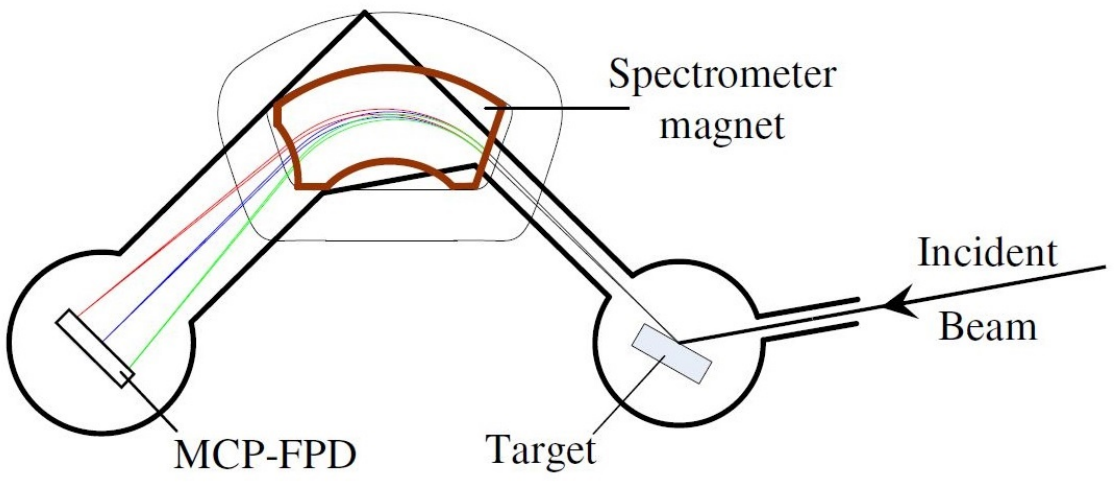

(b) Schematic layout of the HR-RBS detection system. Ions scattered from the target within a fixed scattering angle window enters the spectrometer magnet. Ions with higher momentum will be bent with a larger radii (red) while lower momentum ions will have a smaller radii (green).

Figure 3.11: HR-RBS detector system. Source [5]

The HR-RBS detection system is shown in figure 3.11. The detector system comprises of a $90^{\circ}$ double focusing electromagnet coupled to a multi-channel plate detector [5]. The magnet has a bending radius of $175 \mathrm{~mm}$ with a maximum bending power of $2.1 \mathrm{MeV}$. The function of the electromagnet is to bend the ion beam and spatially segregate the ion beam based on its energy. The MCP detector is then placed on the path of the bent ion beam. The detector is a $100 \mathrm{~mm}$ long and $15 \mathrm{~mm}$ high z-stack multi-channel plate placed in the focal plane perpendicular to the ions (central ion trajectory) exiting the magnet. The MCP 
is connected to a resistive anode readout which measures the position of the ion hitting the $\mathrm{MCP}$ which is directly related to its energy.

In the figure trajectories of particles with energy parameter $\left(\varepsilon=E / E_{0}\right)$ of $0.88,1$ and 1.15 are shown. $E_{0}$ is the energy of the ion passing through the central ion trajectory. It can be seen that the position at which each ion beam strikes the MCP is dependent on its energy. The MCP detector merely measures the position of the scattered ion beam after passing through the electromagnet. This position can be related to the energy of the ion beam by function $f(E)$ described by the following relation,

$$
f(E)=\left.F\left(X\left(E / E_{0}\right)\right) \frac{1}{E_{0}} \frac{d X}{d \varepsilon}\right|_{\varepsilon=E / E_{0}}
$$

where $X$ is the position of the ion. By sweeping the magnetic field to different values the entire energy range of the scattered ions can be covered. The energy is related to the magnetic field as per the relation given in equation 3.5. The magnetic chamber is mounted on rails and can thus be rotated around the scattering chamber to cover various scattering angles. The different possible scattering angles covered by this setup are: 30, 50, 65, 80, 95, 110 and 125 .

In this work, HR-RBS measurements were carried out in the following setup,

Table 3.2: Experimental parameters for HR-RBS setup

\begin{tabular}{|c|c|}
\hline Parameters & Value \\
\hline \hline Energy & $500 \mathrm{keV}$ \\
\hline Ion & $\mathrm{He}^{+}$ \\
\hline Scattering Angle & $65^{\circ}$ \\
\hline Detector resolution & $1.7 \mathrm{keV}$ \\
\hline
\end{tabular}

The effect of ion mass, ion energy and scattering angle are already discussed in detail in section 2.1. The beam diameter was collimated to $1 \mathrm{~mm}$ and the detector resolution for the given setup at the point of measurement was obtained to be $1.7 \mathrm{keV}$. This experimental setup is designed to basically answer the question "At a given scattering angle, how many scattered ions have a particular value of energy?".

So far the instrumentation involved in the HR-RBS measurement are discussed. However, the data obtained from the measurement cannot be directly used for analysis. There are two basic steps that needs to be completed before analyzing the HR-RBS spectrum by a simulation software. The steps are detailed below, 


\subsubsection{Data Stitching}

HR-RBS as mentioned earlier, offers a very high resolution in probing the composition on the surface. This is possible by separating the ion beam scattered from the sample surface under an electromagnet. The scattered ion beam after passing through the electromagnet follows a different path based on their energy. These particles are collected in the multi-channel plate detector. At a particular magnetic field, considering the calibration and setup of the detector, the scattered particles of only a certain energy range can be effectively measured. The magnetic field has to be changed to shift this detection range higher or lower. This requires performing multiple measurement runs of the same sample at different magnetic fields to cover the entire region of interest.

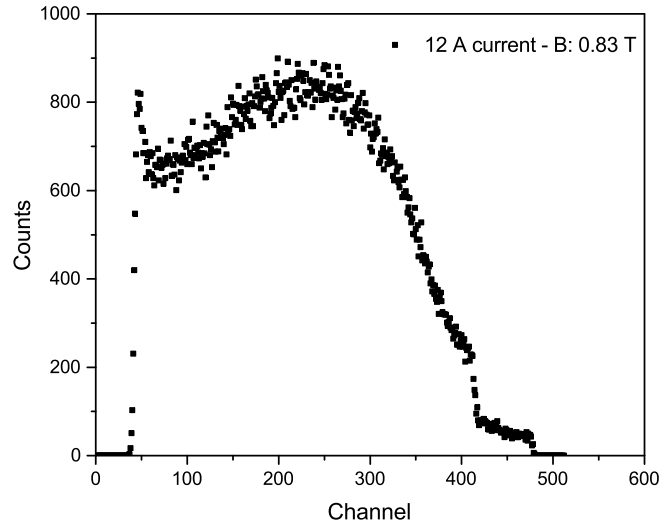

(a) HR-RBS Data of Co implanted DLC film obtained at magnetic field $\mathrm{B}=0.83 \mathrm{~T}$

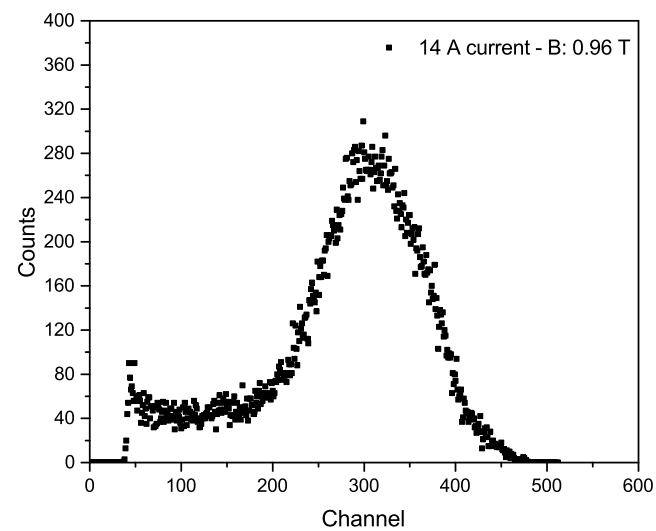

(c) HR-RBS Data of Co implanted DLC film obtained at magnetic field $\mathrm{B}=0.96 \mathrm{~T}$

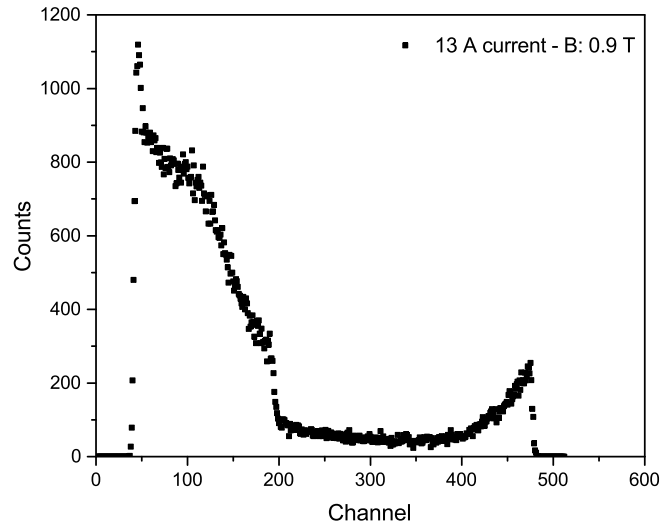

(b) HR-RBS Data of Co implanted DLC film obtained at magnetic field $\mathrm{B}=0.9 \mathrm{~T}$

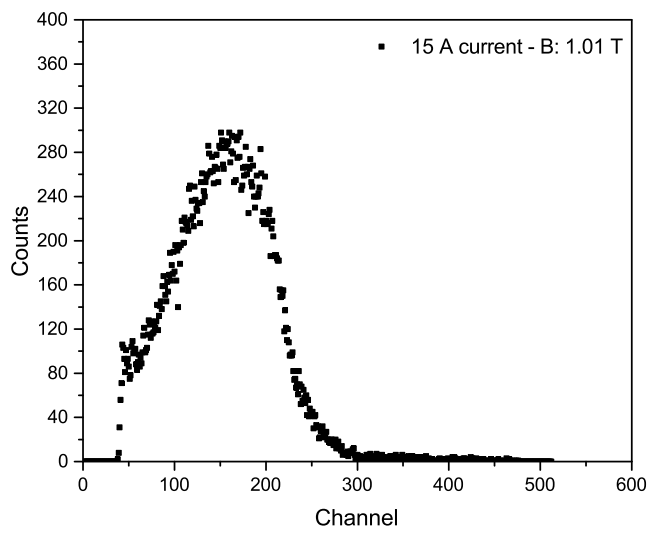

(d) HR-RBS Data of Co implanted DLC film obtained at magnetic field $\mathrm{B}=1.01 \mathrm{~T}$

Figure 3.12: Raw data of sample Co implanted DLC obtained from HR-RBS measurement 
In the above graphs (figure 3.12), the abscissa denotes the channel number and the ordinate shows the number of ions hitting a particular channel. Each graph above shows a spectrum of Co implanted DLC measured at a particular magnetic field. The magnetic field had to be changed from $0.83 \mathrm{~T}$ to $1.01 \mathrm{~T}$ in four steps to cover the entire region of interest. The spectrum obtained at each magnetic field have to be combined in order to extract the depth distribution of Co in the sample. For this purpose a software code was used to stitch the data together to get a unified spectrum which is shown below in figure 3.13.

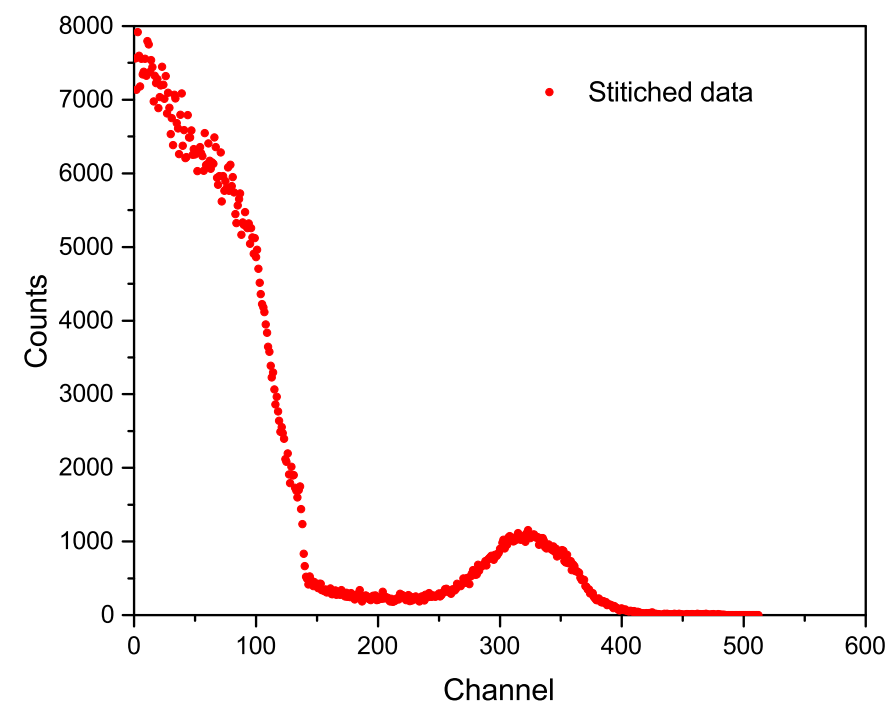

Figure 3.13: Stitched raw HR-RBS data of Co implanted DLC film

\subsubsection{Calibration}

Any measuring device needs to be calibrated before use. Every time an ion beam is setup a standard reference sample needs to be measured to calibrate the energy of the channels at each magnetic field employed. In this case, a $5 \mathrm{~nm}$ tantalum nitride thin films is used as a standard to calibrate the energy scale. Ta and N are present at their surface and the channels at which the Ta and N signals appear in the RBS spectrum can be fit to the corresponding energy which provides the calibration details.

From the kinematics, for a $500 \mathrm{keV}$ beam Ta and N signal should appear at $487.6 \mathrm{keV}$ and $357.3 \mathrm{keV}$. In the calibration measurement, their corresponding signals were recorded at channel number 483 and 183 respectively.

The energy of a particular channel is given by, 
Channel Energy $=$ Calibration Offset + Channel Number $\times$ Energy per Channel

Solving the above as linear equation in two variable, the channel offset and energy per channel is determined to be $277.570 \mathrm{keV}$ and $0.4344 \mathrm{keV} /$ channel. The uncertainty in the calibration is defined by the system resolution of $1.7 \mathrm{keV}$.

\subsubsection{SIMNRA fitting}

In order to extract the elemental distribution from the data spectrum, SIMNRA, a simulation software is utilized [70,71]. The purpose of the software is to simulate an RBS spectrum based on the input parameters. These input parameters consist of both the operational settings associated with the instrument and the compositional information about the target sample. For example, one can construct a target with an assumed concentration and depth profile which can then be simulated as an RBS spectrum based on the operational conditions applied in the experiment. The simulated spectrum can then be compared with the experimental data. The input parameters can iteratively be tuned to obtain a simulated spectrum that aligns with the experimental data. Since the experimental parameters such as ion energy, detection angle are known variables they are assigned a constant value in the simulation. The target composition and depth profile are the unknown data which are continuously refined in the simulation input to obtain the best match between simulation and experiment. The objective of this exercise is to determine the target composition and depth profile that provides the best match between the simulation and experiment. This particular composition and depth profile provides us the information about our sample material.

\subsection{Cross-sectional Transmission Electron Microscopy}

In the previous section the interaction of the target material with energetic ion beams were used to probe their properties, namely elemental distribution and composition. In electron microscopy similar interaction between the target material and energetic electrons are used to probe the material's structure, topology, morphology and composition. The interaction of energetic electrons with target atoms, is similar to ion-atom interactions detailed in section 2.1 and can thus be divided into elastic and inelastic interactions. Elastic interactions are used for transmission electron microscopy and electron diffraction studies.

In TEM, a beam of energetic electrons are directed at a thin section of a target material. The electrons transmitted through the material are collected to image the thin section of 
the target material. Most of the electrons are either not scattered or only scattered by small angles on passage through the thin section. Scattering by high angles or backscattering has a very small cross-section and hence has a low probability for occurrence. However, this probability varies with the atomic number of the atoms composing the target. Those elements with high $\mathrm{Z}$ contain a larger positive charge at its nucleus have greater interactions with the electrons increasing the probability of large scattering events and can also result in broadening of the electron beam. As a general effect, intensity of the direct beam is weakened by deflections of electrons in the forward direction. This leads to a contrast between regions with high and low atomic number atoms. Similar effects are also observed when the density of the material varies at different regions of the target. These effects are exploited in imaging the cross-section of the samples in TEM [72].

In this work, transmission electron microscopy investigations were carried out to locally analyze as-deposited and Co implanted DLC films. TEM was performed using an image Cs-corrected Titan 80-300 microscope (FEI) operated at an accelerating voltage of $300 \mathrm{kV}$ [73]. In particular, cross-sectional bright-field TEM (BF-TEM) micrographs were taken to characterize morphology and microstructure of the DLC films. The electron microscope uses an field emission electron source to generate a high density and highly coherent electron beam. It contains a three condenser lens system to ensure that a nanometer sized parallel beam is generated. The instrument offers a point to point resolution of $0.20 \mathrm{~nm}$ and the information limit is $0.10 \mathrm{~nm}$.

Prior to TEM analysis, the specimen mounted in a double-tilt analytical holder was placed for $10 \mathrm{~s}$ into a Model 1020 Plasma Cleaner (Fischione) to remove organic contamination. TEM lamella preparation was done by in-situ lift-out using a Zeiss Crossbeam NVision 40 system. To protect the surface of the DLC film, a platinum cap layer was deposited beginning with electron beam assisted deposition and subsequently followed by Ga focused ion beam (FIB) assisted precursor decomposition. Afterwards, the TEM lamella was prepared using a $30 \mathrm{keV}$ Ga FIB with adapted currents. Its transfer to a 3-post copper lift-out grid (Omniprobe) was done with a Kleindiek micromanipulator. To minimize sidewall damage, Ga ions with only $5 \mathrm{keV}$ energy were used for final thinning of the TEM lamella to electron transparency. Figure 3.14 shows a typical cross-section prepared by this method. 


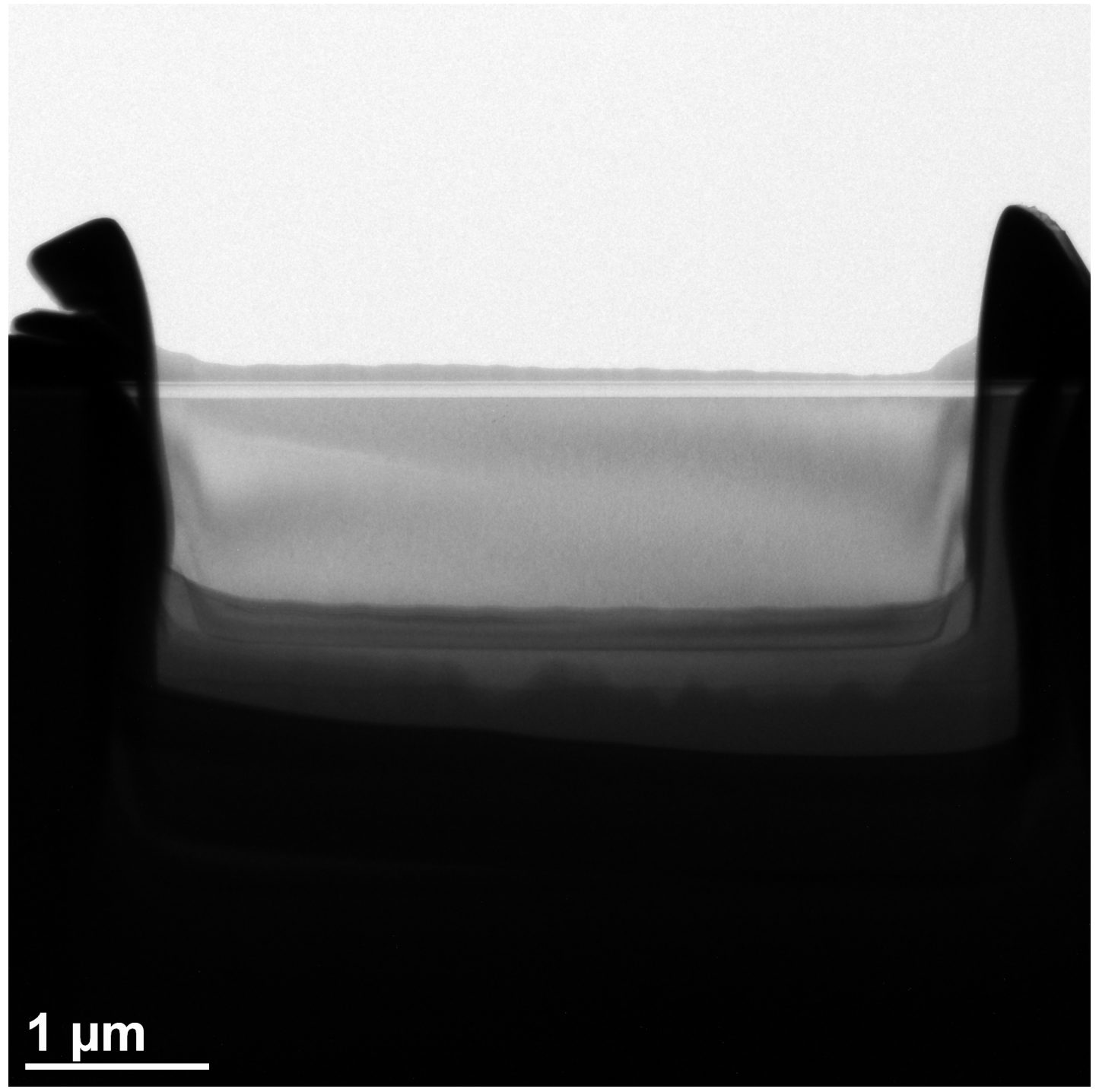

Figure 3.14: TEM lamella of Co implanted DLC film prepared by FIB milling

Presence of any ordered phase within the sample cross-section can be probed by looking for diffraction pattern from the electron image. A coherent beam of electron upon interaction with a material lacking order loses phase uniformity after scattering events. However, in crystalline materials the constituent atoms which acts as scattering centers are distributed in an ordered fashion. Since the electron beam is coherent and the crystalline order of the scattering centers can be extracted from the scattered electron beam. It is easier to extract this information by considering the wave nature of electrons.

Fast Fourier transformation of the imaged sample can represent the scattering centers (atomic positions) in the reciprocal space. Amorphous material do not provide an ordered pattern. While polycrystalline materials result in formation of ring like patterns in reciprocal space due to presence of crystals at different alignments. Single crystals lead to constructive 
interference at specific spots in reciprocal space. Details about the application of FFT analysis can be found in chapter 5 .

The information gathered from elastic interactions pertain to the structural information of the sample. While contrast details differentiate regions of high atomic number from low atomic number they do not provide information about the exact chemical composition of the target. Inelastic interaction of the electrons with the target atoms can provide information about the chemical make up of the target. An energetic electron can ionize a target atom and reduce its energy leading to an inelastic interaction. Ionization leads to an electron vacancy within the atom. If the vacancy lies in the inner electron shell, the atom is energetically unstable. In such cases the outer shell electrons drops down to fill the gap in the inner electronic shell. The difference in energy is released as electromagnetic radiation.

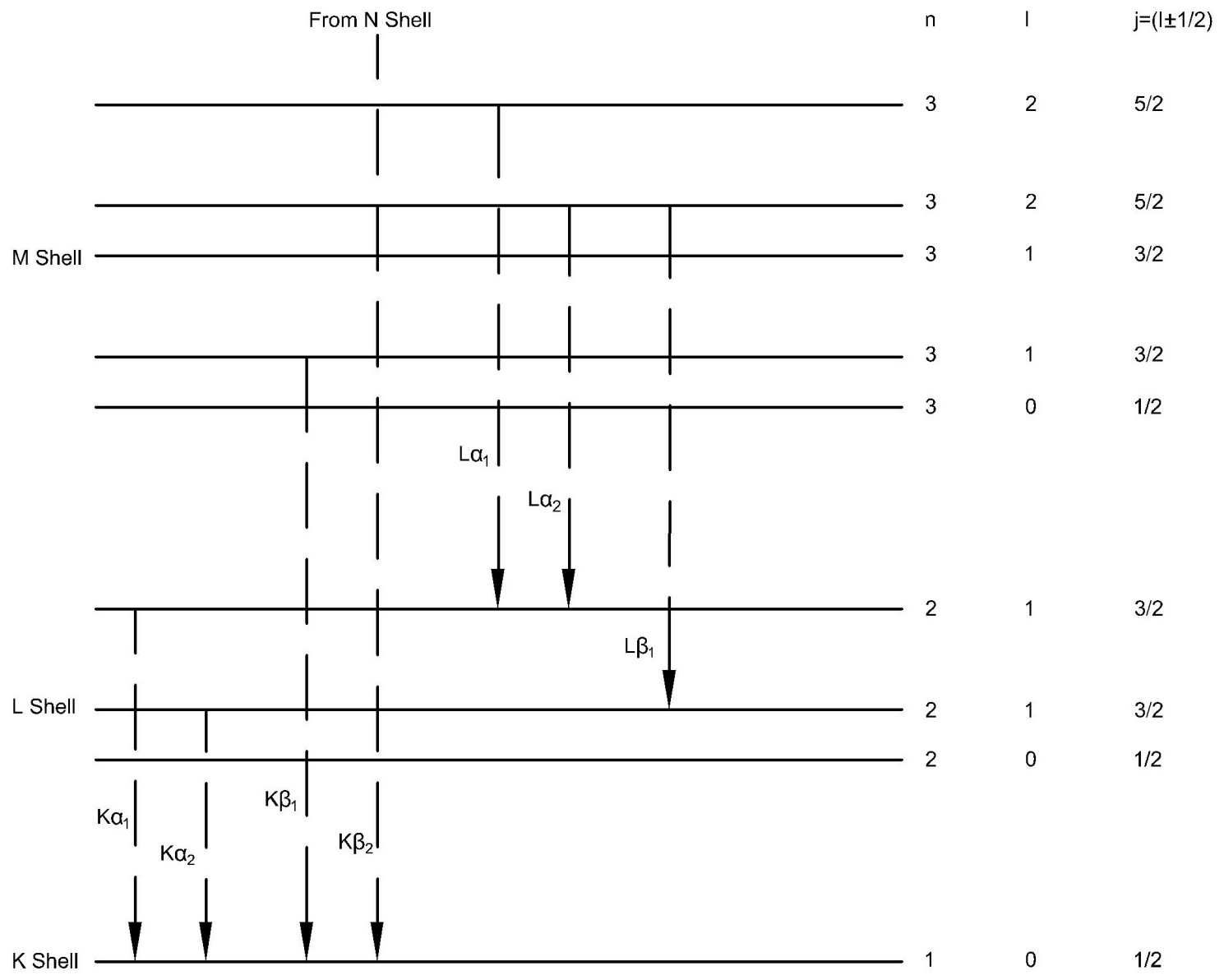

Figure 3.15: Schematic representing transitions in electronic energy levels.

Figure 3.15 shows a schematic explaining this transition. The difference in energy levels results in emission of radiation in the X-Ray regime. The difference in energy level is different for each element and hence the emitted X-Rays are characteristic for that particular 
element. By detecting the energy levels and intensity of the X-Ray emitted from the electron interaction with the target, the chemical composition of the target can be determined. Electron microscopy allows scanning a particular region of the sample (by scanning a line or an area of the sample) to obtain its chemical composition. This technique is referred to as Scanning-TEM (STEM).

\subsection{Resonant Nuclear Reaction Analysis}

Resonant nuclear reaction analysis were carried out on the as-deposited and implanted DLC films to measure the composition and depth profile of hydrogen within the DLC films [74, 75]. HR-RBS and TEM do not provide any direct information about the hydrogen present in the films. Since hydrogen forms a major part of the DLC matrix it is essential to probe its distribution. Of all the available techniques for hydrogen measurement, RNRA is particularly useful as it can provide an accurate quantitative measurement of the hydrogen concentration as function of depth in a non-destructive manner applicable for thin films and coatings with a wide range of thickness [76, 77].

When calculating the distance of closest approach, it was mentioned that for nuclear reactions to occur the projectile has to pass through the electron shielding and penetrate the nucleus. Very high energies are needed for the projectile to approach such distances. Hence, the first requirement for nuclear reaction analysis is an accelerator capable of producing ions with energy in the order of several MeV. Measurement of hydrogen is based on the following nuclear reaction [78],

$$
{ }^{15} \mathrm{~N}+{ }^{1} \mathrm{H} \rightarrow{ }^{12} \mathrm{C}+{ }^{4} \mathrm{He}+\gamma
$$

At the resonance energy of $6.385 \mathrm{MeV}, 15 \mathrm{~N}$ isotopes react with hydrogen producing alpha particles and gamma ray with characteristic energy of $4.43 \mathrm{MeV}$. The samples in this work were measured using a doubly charged $15 \mathrm{~N}$ ions produced by $4 \mathrm{MV}$ Dynamitron Tandem accelerator at RUBION, University of Bochum. The accelerator can produce $15 \mathrm{~N}$ beam with intensities upto $200 \mathrm{nA}$, however the beam current density is reduced to $5 \mathrm{nA}$ to minimize effusion of hydrogen from ion beam exposure. The beam diameter is fixed to $2 \mathrm{~mm}$ by passing it through a collimeter. The entire setup is evacuated by two vacuum pumps: titanium sublimation pump and magnetic bearing turbo-molecular pump supported by oilfree dry roughing pumps to minimize hydrocarbon in the residual gas. This is particularly important for this measurement technique as any hydrogen present during beam exposure can affect the detection limits and error margin of hydrogen in the sample. The sample is 
placed in a UHV chamber in the end of the beamline which is evacuated to $2-3 \times 10^{-9}$ hPa. A 12 in. $\times 12$ in. $\mathrm{NaI}(\mathrm{Tl})$ bore hole detector was used to detect and record the gamma rays, which are proportional to the hydrogen content in the material. The detection limit for hydrogen is limited by the residual background in the detector and the applicable currents. The current setup allows detection of hydrogen upto 100 ppm with measurement time up-to few minutes.

Depth profiles can be obtained by rising the incident $15 \mathrm{~N}$ beam energy above the resonance energy so that the ion has to lose energy passing through the sample before the resonance energy is reached. As the energy of the beam is increased, the resonant reaction occurs in a deeper region in proportion with the increase in beam energy. The resonance width is $\Gamma=1.8 \mathrm{keV}$. Since the resonance cross-section is narrow, hydrogen concentration in a thin section of the target can be probed. The resolution of the measurement is however limited by the experimental setting which results in an energy resolution of $16 \mathrm{keV}$. For DLC films with an atomic density of $1 \times 10^{23}$ atoms. $\mathrm{cm}^{-3}$, the depth resolution corresponds to 10 $\mathrm{nm}$. For a thin film of $150 \mathrm{~nm}$ this resolution increases to $15 \mathrm{~nm}$ at deeper regions due to effects of energy straggling. The samples were measured in steps of $6 \mathrm{keV}$. At each step, data was collected to a charge count of $100 \mathrm{nC}$. The data was collected in the energy range of 6.35 to $6.75 \mathrm{MeV}$. The standard used for the measurement is an amorphous silicon layer that contains 12.5 at.\% of hydrogen. The hydrogen concentration of the samples were calculated in comparison with the standard.

The fraction of hydrogen atoms in each step can be calculated by the formula [75]

$$
H_{\text {sample }}=Y \times K \times \varepsilon_{C} /\left(1+K \times Y \times\left(\varepsilon_{C}-\varepsilon_{H}\right)\right)
$$

where $H_{\text {sample }}$ corresponds to the hydrogen concentration of the sample, $Y$ refers to the gamma yield from the specific sample step after an appropriate background subtraction, $K$ is a constant factor of $K=7.1 * 10^{7} \mu \mathrm{CeV}^{-1} \mathrm{~cm}^{-2}$. $K$ includes the resonance properties as well as the details of the experimental set up and $\varepsilon_{H}$ and $\varepsilon_{C}$ refer to the stopping cross section of the $15 \mathrm{~N}$ beam ions in hydrogen and carbon respectively.

\subsection{Raman Spectroscopy}

The characterization techniques discussed so far involved interactions between ions or electrons with the target material. RBS was based on elastic interaction of ions with the target while RNRA was based on inelastic nuclear interactions. Electron microscopy allowed use of both elastic and inelastic interaction of electrons with the target material to probe its 
structure and composition. Raman spectroscopy is similarly based on inelastic interaction of photons with the target material.

Light usually undergoes elastic interaction with matter. This is commonly referred to as Rayleigh scattering. However, approximately one in a million photons can undergo inelastic scattering with matter and this is referred to as Raman scattering [79]. Raman scattering can provide information about structure, chemical composition, crystalline quality and orientation of a material. In this work Raman spectroscopy is used to qualitatively probe the $\mathrm{sp}^{2}: \mathrm{sp}^{3}$ ratio of the as-deposited and implanted DLC films.

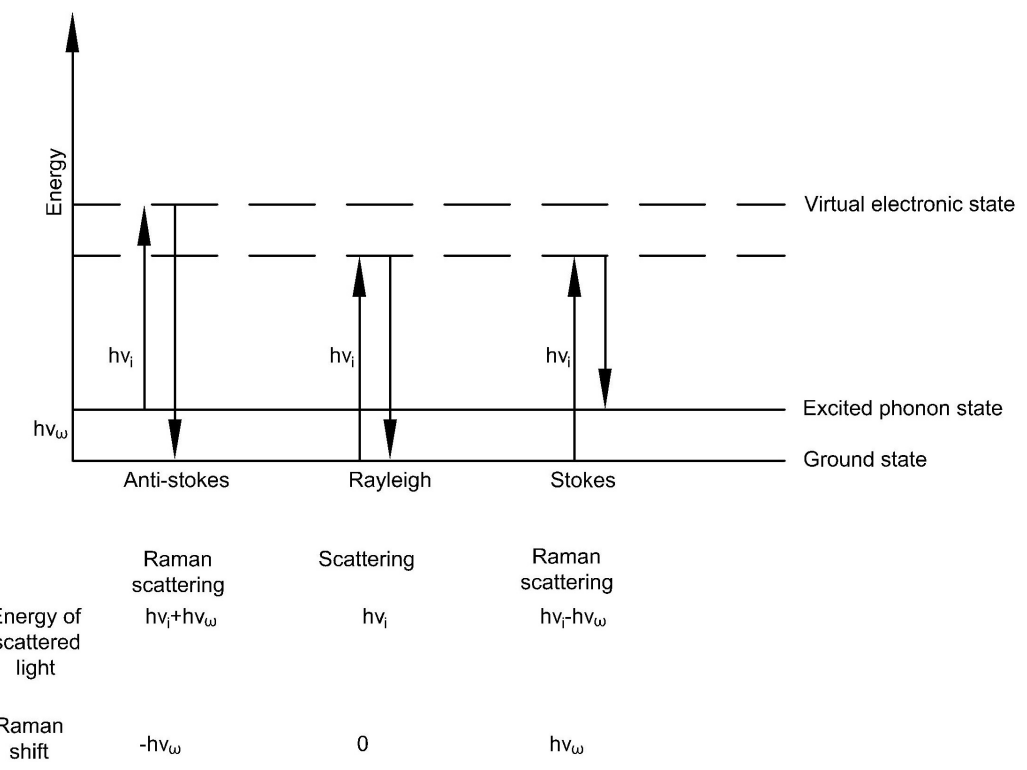

Figure 3.16: Schematic representing excitation of electrons on incidence of electromagnetic radiation. Most electronic excitations result in release of the incident photon without change in energy level. A minor fraction of the electrons after excitation do not relax back to their initial energy level and the difference of energy between the initial energy level and the final relaxed energy level is observed in the photon emitted during relaxation. These transitions are referred to as Raman transitions and probing these transitions provides information about the different phonon states of the target material.

When electromagnetic waves interact with the electrons of a material it causes oscillation of its electric dipole moments to the same frequency as of the radiation. This causes excitement of the atom to a virtual state defined by the frequency of the electromagnetic wave. The excited atom usually relaxes back to its original state by releasing a photon equal to the energy difference between its original state and the virtual excited state as shown in figure 3.16. However, sometimes the incident photon can fundamentally alter the vibrational 
energy level of the atom. This results in relaxing of the excited atom to a level different from its original vibrational energy level. This difference is reflected as change in the energy of the released photon when compared to the incident photon. When the released photon has an energy lower than the incident photon, the atom is said to have undergone a Stokes Raman scattering and when the energy of the released photon is higher than the original photon the atom is said to have undergone an Anti-Stokes Raman scattering. Each of this transition can be described in the following manner respectively,

$$
\begin{aligned}
& h v_{i}-h v_{f}=h v_{w} \\
& h v_{f}-h v_{i}=h v_{w}
\end{aligned}
$$

The shift is measured in $\mathrm{cm}^{-1}$ and corresponds to the allowed modes of vibrations within the material.

The implanted and as-deposited films were characterized by confocal Raman spectroscopy at Victoria University of Wellington following the data interpretation as developed by Ferrari et al. [10]. The setup uses an Ar ion laser (514.5 nm) as an excitation source. A $100 \times$ objective lens was used to focus the laser beam onto the sample. The laser power was limited to $65 \mathrm{\mu W}$ to prevent heating of the sample. The spectrum was collected for $30 \mathrm{~s}$ and each measurement was repeated 5 times to obtain a high signal to noise ratio. The measurements were calibrated with a silicon peak at $521 \mathrm{~cm}^{-1}$. The resolution was found to be less than $1 \mathrm{~cm}^{-1}$. The application of Raman spectroscopy in DLC films will be discussed in detail in chapter 4 .

\subsection{Magnetic property measurement system}

The techniques discussed so far utilized the interaction of energetic ions or electrons with the target material to determine its structure and composition which forms the primary research aim of this work. Another important objective of this research work is to determine the magnetic behavior of Co implanted DLC. For this purpose, a magnetic property measurement system is utilized.

The magnetic behavior of a material can be studied by measuring the magnetic moment developed in the material under application of a magnetic field. In particular, the research

interest lies in measuring the variation in the magnetization of the material with respect to

1. Change in applied magnetic field (from $-7 \mathrm{~T}$ to $7 \mathrm{~T}$ ) at constant measurement temperature. 
2. Change in temperature (2 $\mathrm{K}$ to $350 \mathrm{~K})$ at constant applied magnetic field.

Both these relations are investigated in this research work. The instrument used for this purpose is a magnetic property measurement system (MPMS) based on a superconducting quantum interference device (SQUID) [7]. The instrument is capable of detecting the magnetic moment induced in a sample material. From the magnetic moment, the magnetization and the magnetic susceptibility of the material can be determined.

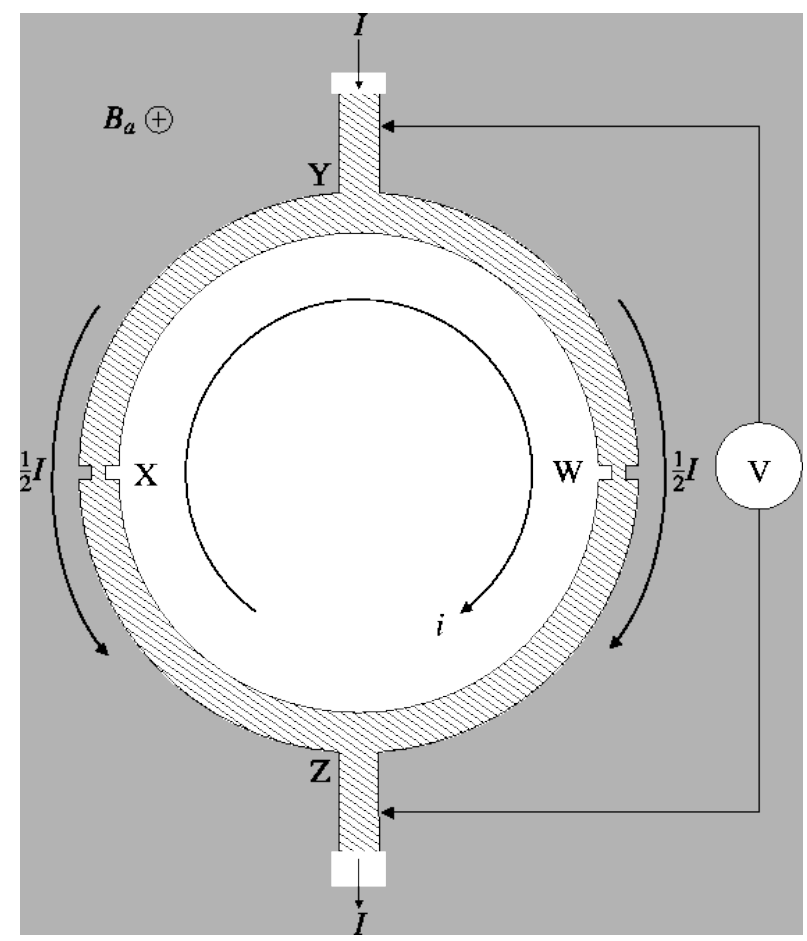

Figure 3.17: Schematic of superconducting quantum interference device composed of a closed superconducting loop with two Josephson junction (X and W). Source [6]

The magnetic moment is detected by the SQUID which is the key component of the MPMS system. Figure 3.17 shows a schematic of the SQUID sensor. The SQUID sensor is composed of a closed superconducting loop including one or two Josephson junctions ( $\mathrm{X}$ and $\mathrm{W}$ in figure). A Josephson junction refers to a set of two superconducting electrodes separated by a non-superconducting barrier. When a measuring current I is passed into the loop, it splits symmetrically in both ends with half of the current through YXZ and other half through YWZ. Any change induced in the SQUID environment (a change in magnetic or electric field) causes flow of a small current inside the SQUID. This current leads to a changing voltage signal proportional to the changing magnetic flux. 


\section{SECOND—DERIVATIVE COIL}

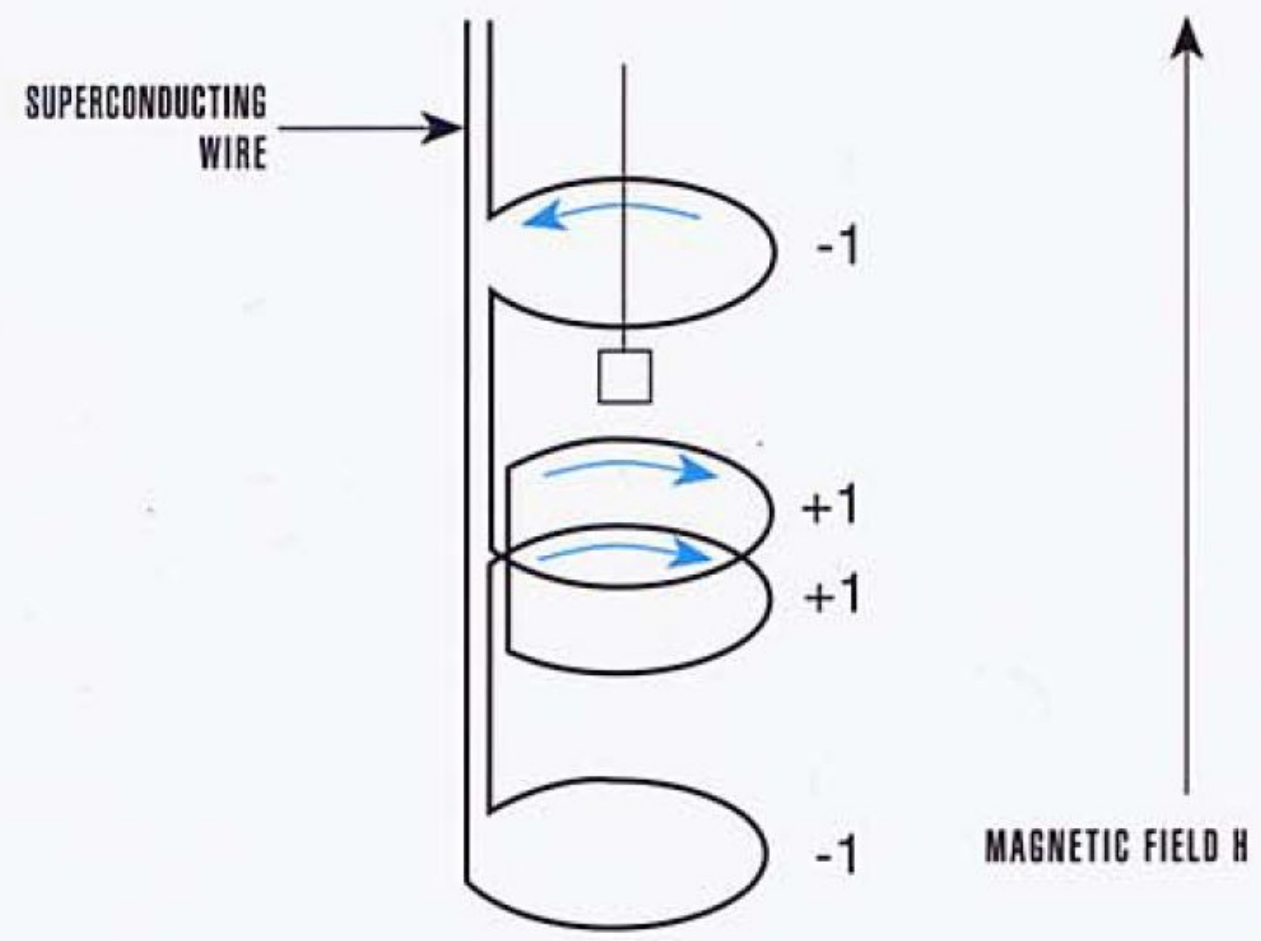

Figure 3.18: Schematic of a SQUID gradiometer used to detect the magnetic moment of the sample. The gradiometer design and operation allows capturing the induced magnetic moment in the sample as an induced voltage in the pick-up coils without interference from the surrounding magnetic fields. Source [7]

The SQUID does not directly measure the induced magnetic flux from the sample but rather is inductively coupled to a 2 nd order gradiometer which measures the magnetic flux generated by the sample. Figure 3.18 shows the schematic of the gradiometer setup employed for measuring the sample dipole response. The gradiometer is a single piece of superconducting wire wound into a set of three coils as shown in the figure. The top and bottom coil consists of a single loop wound in anti-clockwise direction. The center coil consists of two loops wound in the clockwise direction. The superconducting detection coil is extremely sensitive to the surrounding magnetic fields. The sample is loaded in side the gradiometer. This whole setup is placed inside a superconducting solenoid magnet. As the sample is moved inside the gradiometer, the magnetic flux from the sample generates a signal on each of the wire loop as it passes through them.

It is to be noted that the gradiometer by design do not uptake signals from the external magnetic field. The effect of external magnetic field on the clockwise and anti-clockwise 
wound coils will be equal but opposite in magnitude. Thus any signals generated by the external magnetic field get canceled out due to the equal number of clockwise and anticlockwise loops of the detection coil. On the other hand, the sample being small in dimensions generates signal only in those regions of the gradiometer that are in immediate vicinity to the sample. Thus as the sample moves through the gradiometer the specific dipole moment induced in the sample can be measured.

The SQUID measures the moment induced by the sample by relating these signals generated by the sample into a corresponding change in the voltage given by [7],

$$
V(z)=V_{0}+\alpha z+N_{v} m_{z}\left(2 \Omega_{0}-\Omega_{+}-\Omega_{-}\right)
$$

In the above equation, $V_{0}$ and $\alpha z$ compensate for constant and linear measurement offset, $N_{v}$ is the SQUID voltage calibration, and $m_{z}$ is the $\mathrm{z}$ component of the dipole moment of interest. The terms $0,+$, and - represent the signal induced in the gradiometer coils at zero, positive, and negative $\mathrm{z}$ offsets respectively.

The current setup allows measuring a magnetic moment as small as 5 pemu. Detailed information about the setup and the measurement system can be found in [7].

\subsection{Four terminal transport measurement}

Electrical conductivity of implanted DLC is expected to shed more light on the effect of Co implantation onto DLC. For this purpose, a four terminal method is utilized for measuring the electrical resistance in the sample.

In a four terminal method, four contacts are applied onto the sample as shown in figure 3.19. Current is passed through the outer two terminals and the inner two terminals are connected to a voltmeter to measure the voltage developed within the material due to the passage of the excitement current. Resistance of the sample within the inner two terminals can thus be obtained by application of Ohm's law on the measured voltage.

For this work, the measurements are carried out in a physical property measurement system (PPMS) in the DC transport mode. The PPMS from Quantum Design provides a precise control over the excitement current and accurately measures the temperature of the sample and voltage induced in them. Figure 3.19 shows Co 7 sample loaded with four silver contact on top of the sample. 


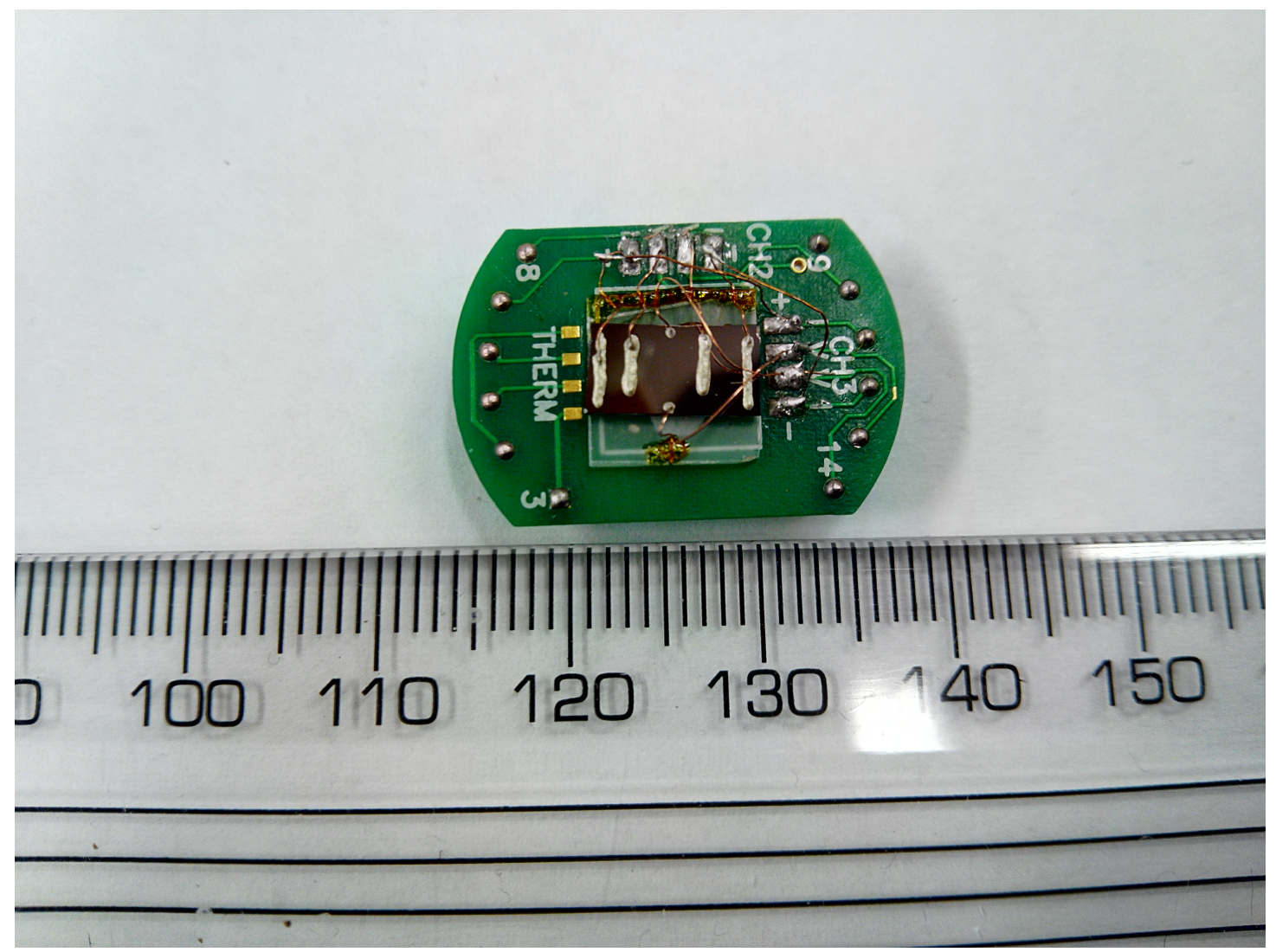

Figure 3.19: Co implanted DLC films on Si substrate loaded for four terminal transport measurement.

Each contact of the sample is connected to the contact on the puck by a thin non-insulated copper wire. The puck is then loaded into the sample holder and is placed inside the PPMS system where controlled transport measurements are carried out. In this work, an excitation current of $100 \mu \mathrm{A}$ was used and the measurement temperature was varied from $2 \mathrm{~K}$ to 350 K. 


\section{Chapter 4}

\section{Ion beam deposition of diamond like carbon}

\subsection{Introduction}

This chapter presents the synthesis of DLC films by direct and mass selective ion beam deposition. The chapter begins with a brief introduction to DLC followed by a discussion on the growth mechanism of DLC. Several mechanisms proposed so far are briefly discussed. The next section presents the details of DLC synthesis by mass selective and direct ion beam deposition used in this work. The properties of these films are presented based on the results obtained from Raman spectroscopy, resonant nuclear reaction analysis, Rutherford backscattering spectrometry and transmission electron microscopy. The chapter is concluded with a discussion on the effect of ion energy (and ion species) on the property of the deposited DLC films.

\subsection{Diamond-like carbon}

Carbon exists in many forms and in different hybridization. Figure 4.1 shows the different hybridization states of carbon. Graphite and diamond are two of its most popular allotrope. Graphite is made of $\mathrm{sp}^{2}$ hybridized carbon and it assumes hexagonal structure. It is a soft material and is a good conductor of electricity. Diamond on the other hand is composed of $\mathrm{sp}^{3}$ hybridized carbon and it assumes a tetrahedral arrangement. It is one of the hardest materials available in nature and is an electrical insulator / wide band gap semiconductor. The mechanical properties of diamond make it a valuable material for industrial applications. Thus the properties of carbon vary greatly depending on its hybridization and its consequent 
structural arrangement.
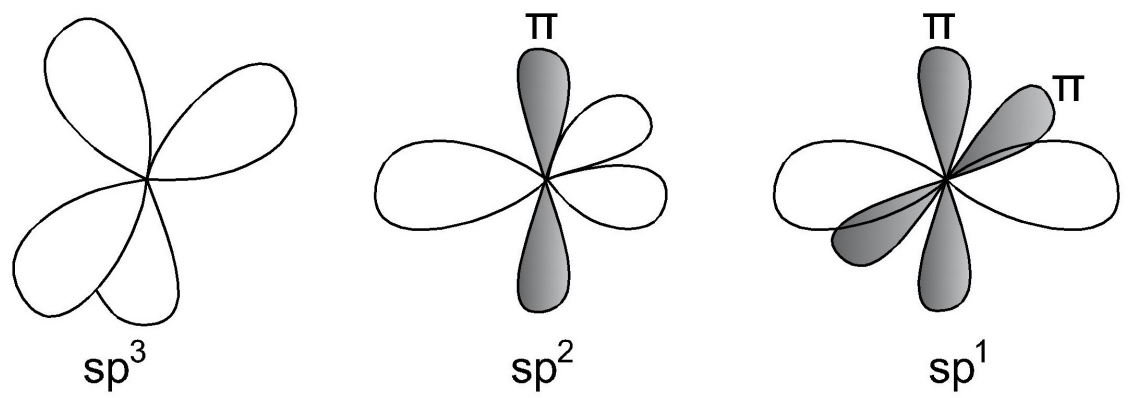

Figure 4.1: Schematic representing the $\mathrm{sp}^{3}, \mathrm{sp}^{2}$, sp hybridized bonding. Redrawn from [8]

Diamond is not commonly available and synthesis of diamond is an expensive process making it commercially nonviable for many applications. The high cost is associated with the extreme conditions required for the synthesis process. At room temperature and normal pressure, graphite or $\mathrm{sp}^{2}$ hybridization is the most stable form of carbon. Formation of $\mathrm{sp}^{3}$ hybridization requires the carbon atoms to pass through a high energy barrier which is feasible only at high pressure and high temperature (HPHT) conditions. Diamond-like carbon serves as an excellent substitute for diamond in industrial applications [80]. It is widely used in the coating industry for its hardness, wear-resistance and slickness. DLC refers to amorphous carbon material with significant fraction of its carbon bonded in $\mathrm{sp}^{3}$ hybridization. The $\mathrm{sp}^{3}$ content directly determines the hardness of the material and it can range from $20-100 \%$ (carbon films containing less than $20 \% \mathrm{sp}^{3}$ content are not generally referred to as DLC) [8]. Figure 4.2 shows the different types of DLC.

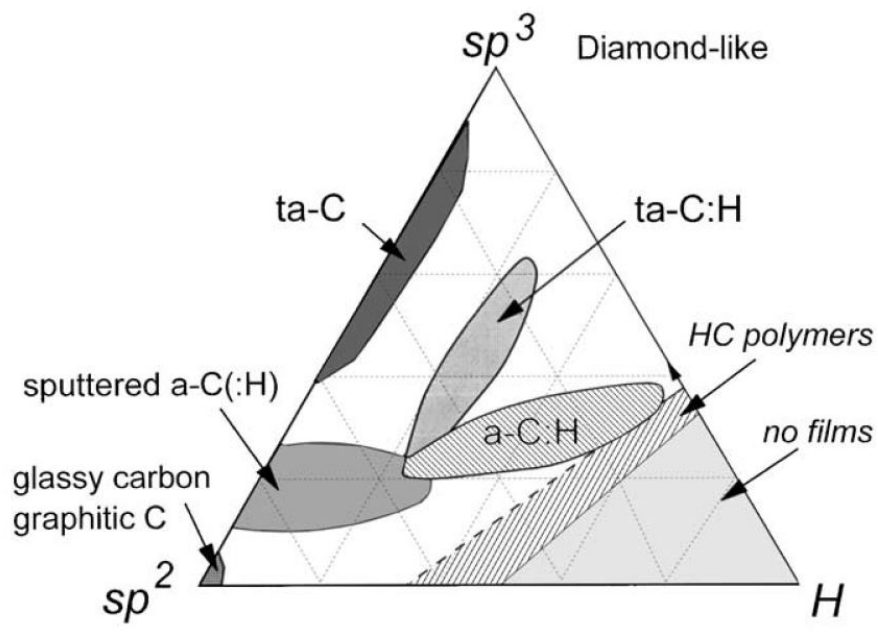

Figure 4.2: Ternary phase diagram of bonding in amorphous carbon-hydrogen alloys. The figure classifies different types of DLC based on their hydrogen configuration and $\mathrm{sp}^{2}: \mathrm{sp}^{3}$ ratio. Source [8] 
The figure shows the different classification of diamond-like carbon. A key feature to be noticed is use of hydrogen content as a variable in the ternary phase diagram. Hydrogen saturates the dangling bonds of carbon which prevents then from assuming $\mathrm{sp}^{2}$ hybridization. Hence hydrogen is generally incorporated during the synthesis process and the hydrogen content directly affects the hardness of DLC. In this work, the term DLC refers to hydrogenated diamond-like carbon and it is the base material used for implantation. As mentioned earlier, DLC can have a range of $\mathrm{sp}^{3}$ hybridization and is relatively inexpensive to fabricate. The extreme conditions required for $\mathrm{sp}^{3}$ formation is met by use of energetic atoms/ions for deposition. The next section discusses the different growth models for DLC fabrication.

\subsection{Growth mechanism}

The key factor responsible for the diamond like properties is the $\mathrm{sp}^{3}$ bonding between neighboring carbon atoms. However, the $\mathrm{sp}^{3}$ bonded state of a carbon atom can only be formed under high temperature and pressure. The most stable form of carbon under normal temperature and pressure is its $\mathrm{sp}^{2}$ hybridization.

In the timeline of DLC research, various growth mechanisms were proposed to explain the foundation of $\mathrm{sp}^{3}$ bonded carbon in DLC films. The process of DLC growth was referred to as "subplantation" denoting the low energy subsurface implantation of the carbon atoms required for growth of DLC films. Spencer et al. proposed that $\mathrm{sp}^{3}$ sites arise from a mixture of $\mathrm{sp}^{2}$ and $\mathrm{sp}^{3}$ bonded carbon by the process of preferential sputtering of the $\mathrm{sp}^{2}$ sites by the energetic ions [81]. However, Lifshitz et al. disproved this model by citing the low sputtering yield of carbon on carbon [82, 83]. It was further noted that the sputtering yield depends on the binding energy of carbon which is almost similar for both $\mathrm{sp}^{2}$ and $\mathrm{sp}^{3}$ carbon. Lifshitz et al. alternatively suggested that enrichment of $\mathrm{sp}^{3}$ carbon arises from preferential displacement of $\mathrm{sp}^{2}$ bonded carbon.

However, later measurements of the displacement threshold for graphite $(35 \mathrm{eV})$ and diamond $(37-47 \mathrm{eV})$ revealed that the difference in displacement energies are not significant enough to justify the enrichment of $\mathrm{sp}^{3}$ sites during ion bombardment. This was later replaced by a model from McKenzie and Davis, where they proposed that ion bombardment creates a compressive stress within the carbon film promoting $\mathrm{sp}^{3}$ bonding [84, 85]. This model can be illustrated better by referring to the Berman-Simon line [9]. Figure 4.3 shows the phase diagram for carbon. The figure shows the diamond phase is stable only at higher pressures (above the Berman-Simon line). McKenzie and Davis suggested that the stress induced during ion bombardment will move the film above the Berman-Simon line resulting 
in stabilization of the diamond phase. Once the phase is created, it is quenched in that phase during the growth of the DLC film.

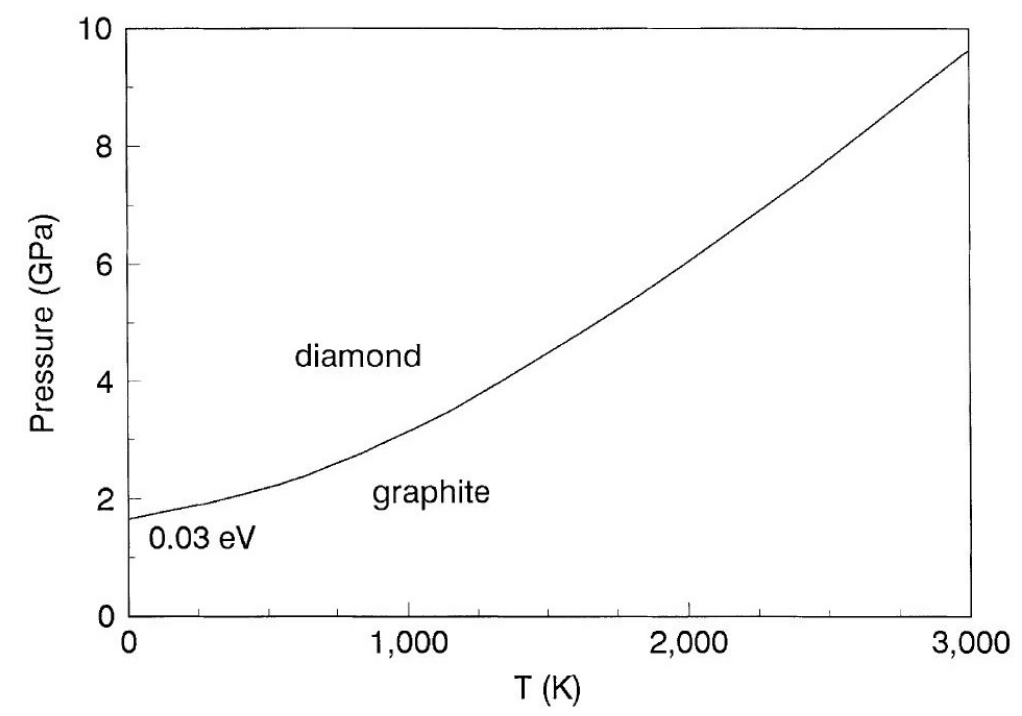

Figure 4.3: Berman-Simon phase diagram for carbon representing the conditions required for synthesis of diamond. Source [9]

The latest and the most comprehensive model for DLC growth was proposed by Robertson $[86,87]$. In his model, he proposes that subplantation causes a metastable increase in the density which leads to transformation of the local bonds to $\mathrm{sp}^{3}$ hybridization. Robertson explains the subplantation process in three stages:

Stage A A collisional stage of $10^{-13} \mathrm{~s}$

Stage B A thermalization stage of $10^{-12} \mathrm{~s}$ and

Stage C A relaxation stage after $10^{-10} \mathrm{~s}$

Of these, stage A promotes $\mathrm{sp}^{3}$ bonding while stage $\mathrm{B}$ and $\mathrm{C}$ causes loss of $\mathrm{sp}^{3}$ bonding which is expected to be significant at high ion energies. This ion beam process is illustrated as a schematic in figure 4.4 and 4.5 . 


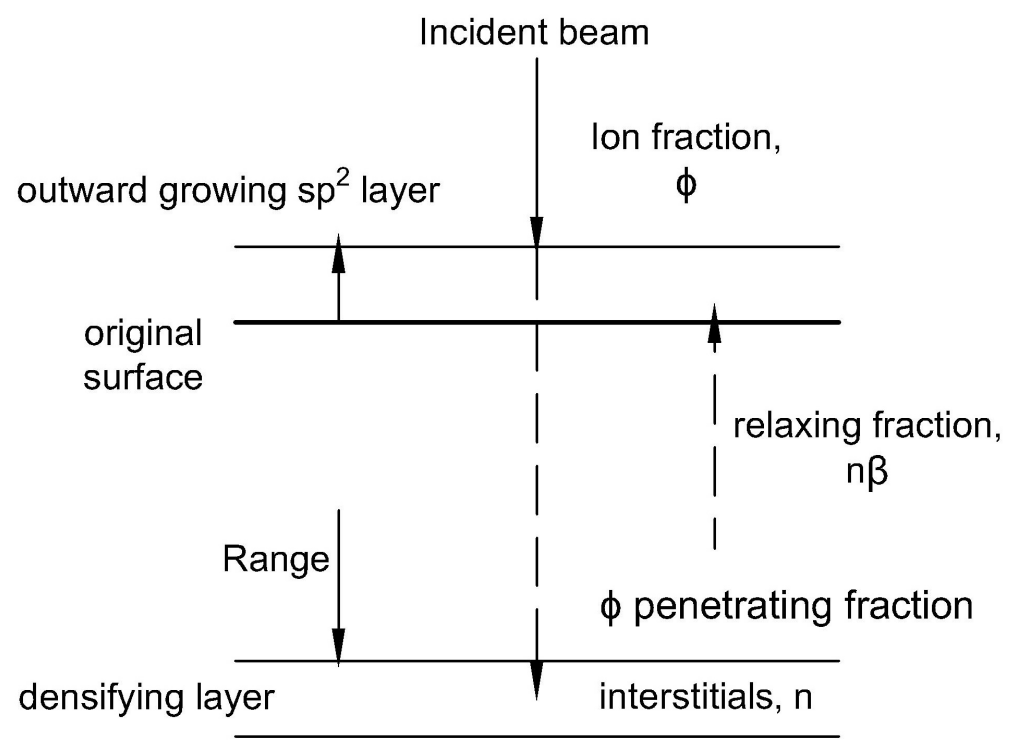

Figure 4.4: Schematic diagram of densification by subplantation. An incident carbon / carbon-rich beam upon implantation introduces carbon (implanted) interstitials at end of its range causing layer densification which is favorable for formation of $\mathrm{sp}^{3}$ hybridization. Those carbon atoms that relax back to surface do not experience the pressure available at the densified layer and hence grows an $\mathrm{sp}^{2}$ rich surface. Redrawn from [8]

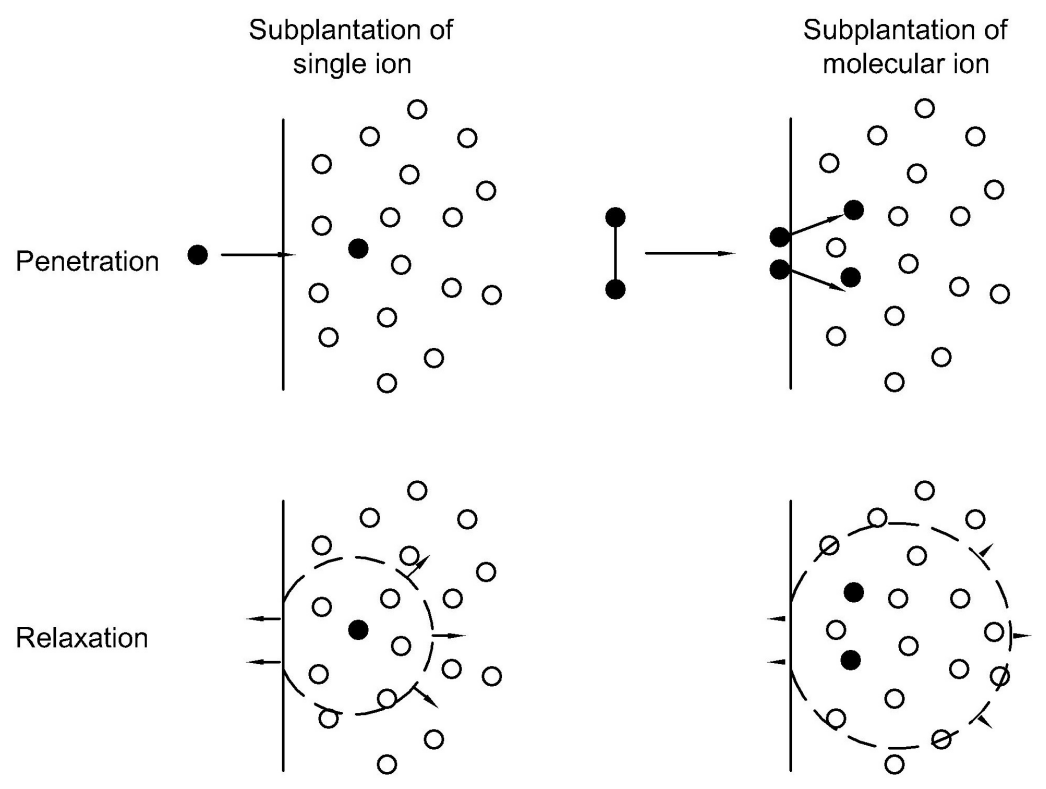

Figure 4.5: Schematic representing subplantation of single and molecular ion. It can be seen that use of molecular ion expands the cascade region caused during implantation. Redrawn from $[8]$

The metastable increase in density was quantified by Robertson [8] as 


$$
\frac{\Delta \rho}{\rho}=\frac{\phi}{1-\phi+0.016\left(E_{i} / E_{0}\right)^{5 / 3}}
$$

where $\rho$ denotes the density, $\phi$ denotes the dose of ions, $E_{i}$ denotes the incident energy (after collisions) and $E_{0}$ the activation energy for diffusion.

The model based on this concept of thermal spike however faces many criticisms. One of the major concern lies in the fact that the deposition process happens at very low energy and involves ions with low atomic masses where the thermal spike has least effect. However, the model provides a good starting point for convergence of theoretical expectations and experimental observations. Further revisions and refinements of the above discussed models can be found in $[88,89]$.

\subsection{Mass-selective Ion beam deposition}

The DLC films used in this research were primarily produced by mass-selective ion beam deposition. In this section, the growth of DLC films by MSIBD [36] and their corresponding properties will be discussed.

The DLC films were grown in a low energy ion implanter using butane as a precursor gas (Section 3.3). The primary ion species selected for deposition was $\mathrm{C}_{3} \mathrm{H}_{6}^{+}$. The deposition was carried out at $5 \mathrm{kV}$ acceleration voltage with an ion current density of $2 \mu \mathrm{A} . \mathrm{cm}^{-2}$. The molecular ion upon impact dissociates into its atomic constituents. The energy of the ion will be divided among the atomic constituents based on their mass. Thus, each carbon atom will have an energy of $1430 \mathrm{eV}$ and each hydrogen atom will have an energy of $120 \mathrm{eV}$. The interaction of these energetic atoms with the Si substrate can be discussed from the ion-solid interactions presented in chapter 2. The key parameters involved in this process are distance of closest approach, reduced energy, nuclear stopping, electronic stopping, projected range, straggling and sputtering. Table 4.1 records these values for both carbon and hydrogen atoms on silicon. 
Table 4.1: Key ion interaction parameters for DLC deposition

\begin{tabular}{|c|c|c|}
\hline Parameters & Carbon & Hydrogen \\
\hline \hline Distance of closest approach & $0.121 \mathrm{~nm}$ & $0.174 \mathrm{~nm}$ \\
\hline Reduced energy & 0.115 & 0.095 \\
\hline Nuclear stopping & $103 \mathrm{eV} / \mathrm{nm}$ & $2.2 \mathrm{eV} / \mathrm{nm}$ \\
\hline Electronic Stopping & $36 \mathrm{eV} / \mathrm{nm}$ & $8.5 \mathrm{eV} / \mathrm{nm}$ \\
\hline Projected Range & $6.6 \mathrm{~nm}$ & $3.1 \mathrm{~nm}$ \\
\hline Straggling & $5.1 \mathrm{~nm}$ & $4.7 \mathrm{~nm}$ \\
\hline Sputtering & 0 & 1 \\
\hline
\end{tabular}

It can be seen from the table that the collision of carbon and hydrogen on silicon substrate is a low energy process (epsilon values). At such energy regime, the primary mode of energy loss is nuclear stopping. The carbon and hydrogen ions is computed to have a mean projected range of $6.6 \mathrm{~nm}$ and $3.1 \mathrm{~nm}$ respectively. However, as can be seen from the table, they have a large straggling of about $5 \mathrm{~nm}$. Figure 4.6 shows their respective projected range in the silicon substrate. This provides an estimate on the extent to which carbon and hydrogen atoms interact with Si matrix.

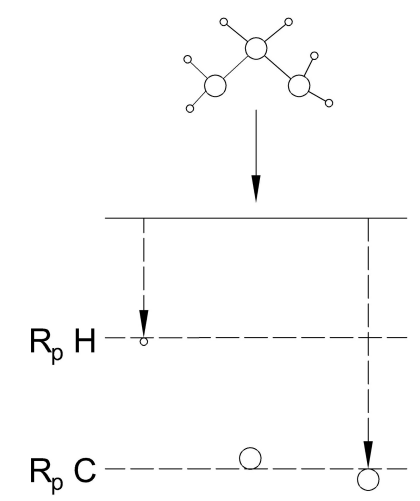

Figure 4.6: Schematic representing difference in $\mathrm{C}$ and $\mathrm{H}$ penetration range into Si substrate

The impact of the ion beam on the silicon substrate can result in three possible outcomes.

1. A net loss of atoms due to domination of sputtering effects

2. A net gain of atoms due to implantation (or in this case, subplantation)

3. No net change due to balance between sputtering and implantation.

For growth of DLC films, the deposition process should result in net gain of carbon atoms. This condition is satisfied in this deposition process. The sputtering yield of carbon on silicon at $1.43 \mathrm{keV}$ is calculated to be $\sim 1$. On the other hand, under the same conditions, 
the sputter yield of carbon on carbon is $\sim 0.3$. This implies a net growth in carbon atoms and a net loss of Si atoms.
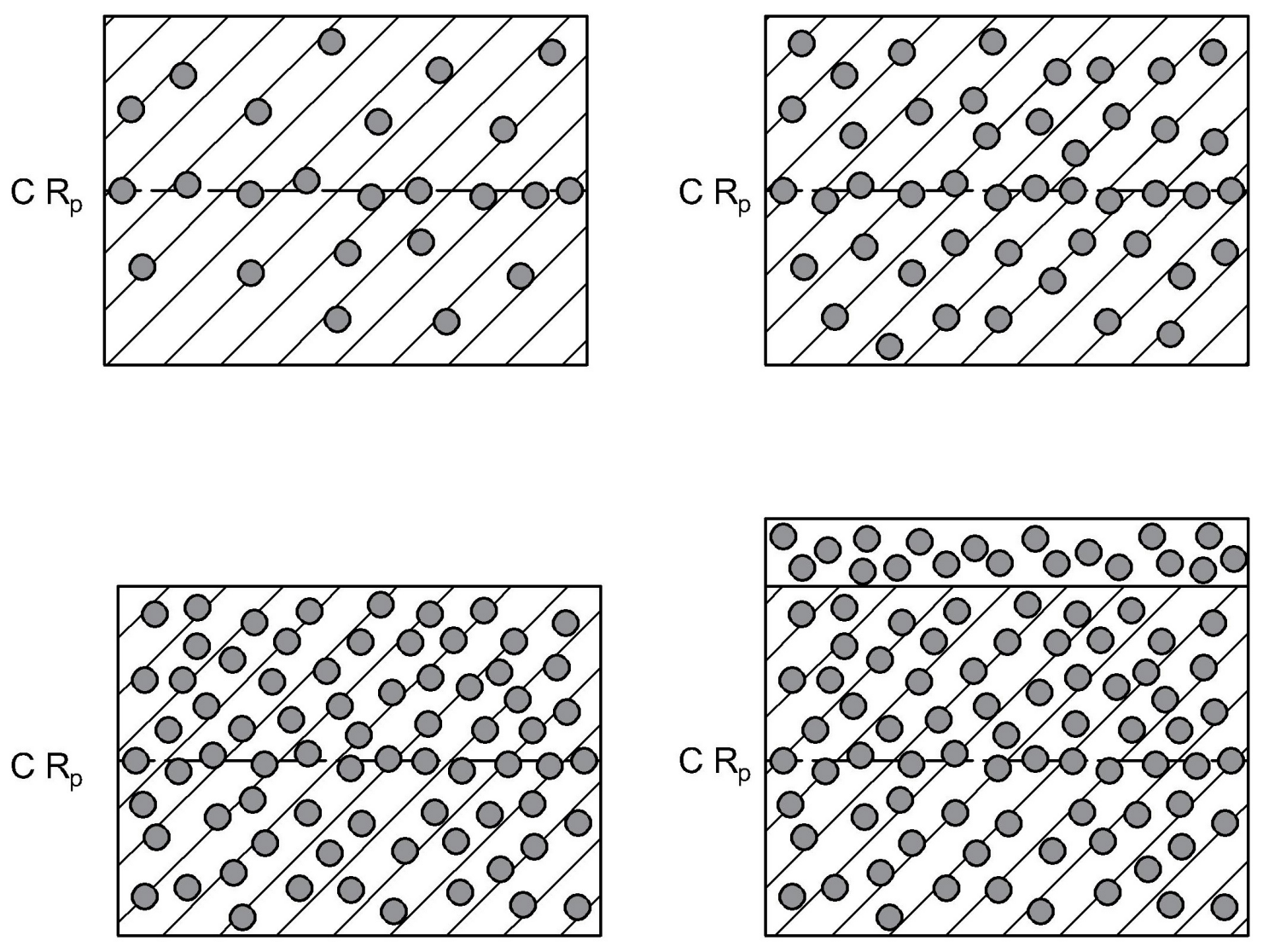

Figure 4.7: Schematic representing growth of DLC by MSIBD. During the initial stage of growth, the carbon atoms are concentrated near the projected range. As the deposition continues the concentration of the carbon atoms in the Si substrate increases further to the point of saturation. As the process continues further increase in carbon concentration results in outgrowth of carbon atoms onto the surface resulting in formation of a carbon layer.

The growth process is shown schematically in figure 4.7. The growth proceeds predominantly from the mean projected range of the carbon atoms. At this point it is important to distinguish the effects of hydrogen and carbon atoms. Carbon atoms are responsible for the growth of the films. Hydrogen atoms saturate the dangling bond of carbon atoms promoting $\mathrm{sp}^{3}$ hybridization. Even though the hydrogen fraction is greater than the carbon fraction from the molecular beam, most of the impringing hydrogen form $\mathrm{H}_{2}$ molecules and effuse out of the film. The retained hydrogen content will be discussed in detail in the resonant nuclear reaction analysis section.

Figure 4.7 shows a schematic for the growth process occurring during ion beam deposition of DLC films. It has to be mentioned here that this is a special condition and is not possible 
for other cases (for example, $\mathrm{Si}$ on $\mathrm{Si}$ ). In most cases, high fluence implantation will be limited by steady-state condition where the number of ions getting implanted will equal the number of atoms getting sputtered, resulting in a dynamic equilibrium. The highest concentration of the implanted ions will be achieved in the projected range and the surface concentration of the implanted ion never approaches 100 at.\% (as sputter yield of implanted ion on implanted atom is usually $>1$ ). However, carbon is special in this manner. The sputter yield of carbon on carbon never approaches unity. This leads to saturation of carbon in the implanted region. Further implantation shifts the mean projected range towards the surface and results in an outward growth of carbon atoms as seen in figure 4.7. This leads to the growth of DLC films. Both MSIBD and DIBD exploits this special nature of carbon to grow carbon films.

Now that carbon growth is explained, the state of implanted carbon inside the substrate can be briefly discussed. While there exists different view points on the growth mechanism, it is safe to assume that the very nature of ion implantation process is that it is a non-thermal equilibrium process. Carbon atoms upon implantation experience compressive stress and enters the spike regime just near the end of its range. Near the end of its range the energy of the carbon atoms is very low leading to a huge increase in its cross-section for collision. This results in a collision cascade. For a very short period of time the temperature and pressure in the cascade region is severely high and can lead to the formation of metastable $\mathrm{sp}^{3}$ phase. The $\mathrm{sp}^{3}$ carbon atom thus formed is quenched in their state as the system relaxes rapidly in the time scale of few picoseconds. This model holds true in the current scenario as well. The formation of $\mathrm{sp}^{3}$ hybridized carbon is evidenced from the Raman analysis that will be discussed shortly.

One important factor associated with the range of carbon during the growth process is its interaction with $\mathrm{Si}$ substrate. While $\mathrm{Si}$ is expected to be sputtered out during the process, as the Si concentration drops to a low value the number of Si atoms effectively sputtered during the deposition process is reduced leading to a dilute concentration of Si still present in DLC. The concentration of Si in DLC is significant in the region immediately close to $\mathrm{Si}$ substrate. This can be seen effectively in the TEM pictures. 


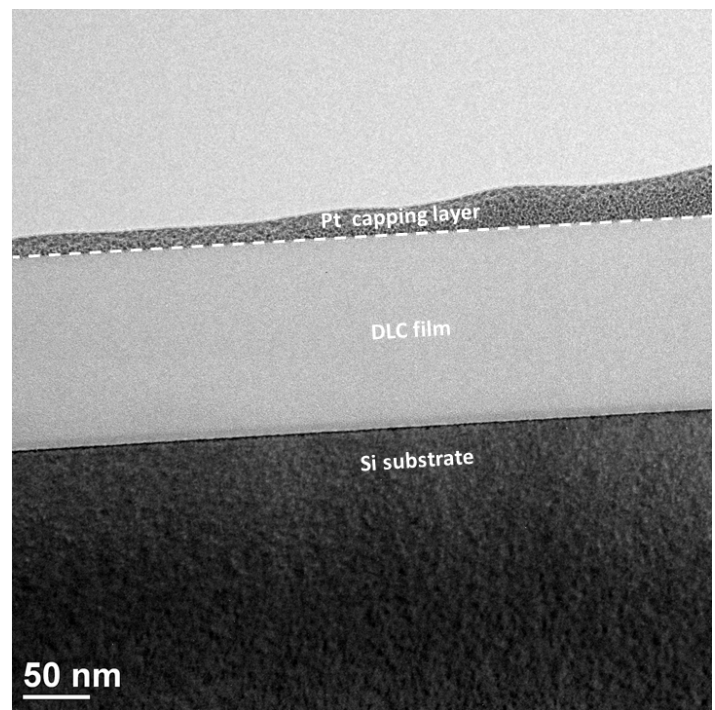

(a) Overview of the cross-section of MSIBD prepared DLC films on Si substrate with $\mathrm{Pt}$ capped layer on top surface.

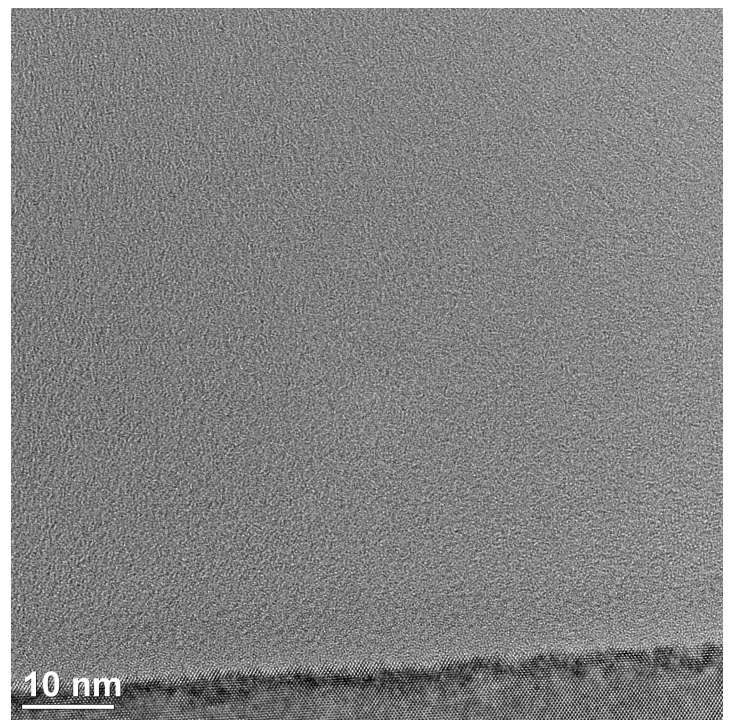

(c) MSIBD deposited DLC thin film / Si substrate interface showing the transition from crystalline silicon layer to amorphous carbon film.

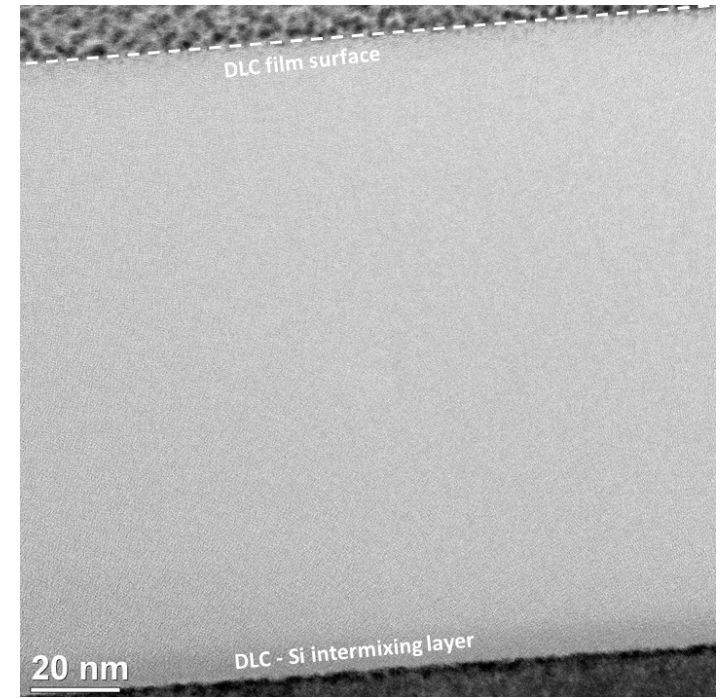

(b) Cross-sectional TEM image showing the surface and interface of the DLC film. The intermixing $\mathrm{Si}$ and $\mathrm{C}$ layer can be clearly seen near the Si interface at the bottom of the picture.

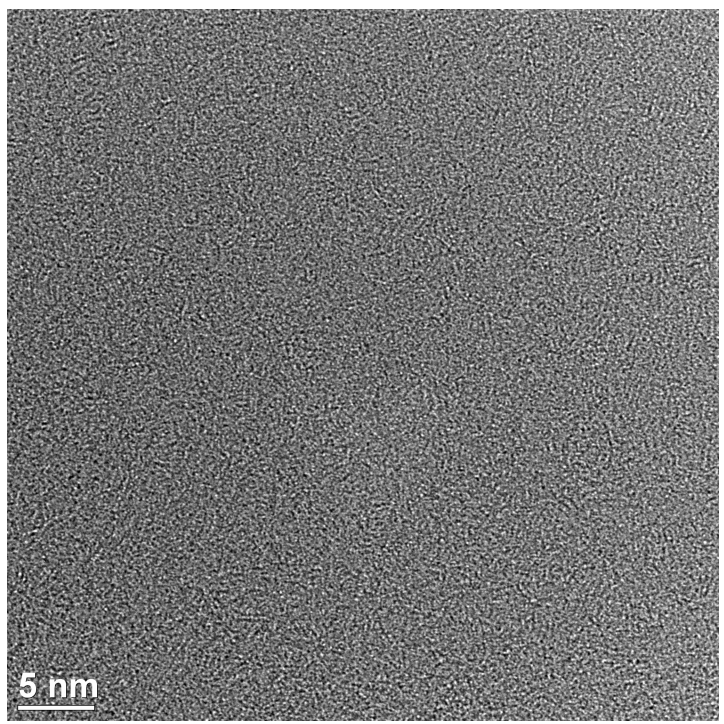

(d) MSIBD deposited DLC thin films showing the amorphous nature of the carbon atoms in the DLC film

Figure 4.8: Cross-sectional TEM images of MSIBD deposited DLC at different length scales

Figure 4.8 shows the TEM pictures of MSIBD deposited DLC films. It can be seen from figure 4.8 (a) that the deposition is uniform. This particular film is about $150 \mathrm{~nm}$ thick. Figure 4.8 (b) shows the same film at a higher resolution. The intermixing $\mathrm{C}$ and Si layer is distinctly visible in this picture. The extent of intermixing as mentioned before is determined by the range of carbon ions which in turn depends on the deposition energy. 
If this energy increases, the extent of intermixing will increase accordingly. Figure 4.8 (c) focused on the $\mathrm{C} / \mathrm{Si}$ interface. The picture clearly shows the nature of the interface. The silicon region is structurally ordered. Near the immediate vicinity of the carbon layer, the $\mathrm{Si}$ regions appears to be densified. This can be attributed to the carbon atoms present beyond the projected range during the start of the deposition process. The carbon atoms present as interstitials leads to the densification process. However the concentration of carbon at these regions is not high enough to cause amorphization. As soon as the concentration increases beyond a threshold, the Si surface loses their structural order leading to amorphous mix of Si and C. This further explains the dense layer surrounding the Si substrate. The DLC film is observed to be completely amorphous. A high resolution image of the DLC film (away from the interface) is shown in figure 4.8 (d). The picture proves the absence of any long range order within DLC.

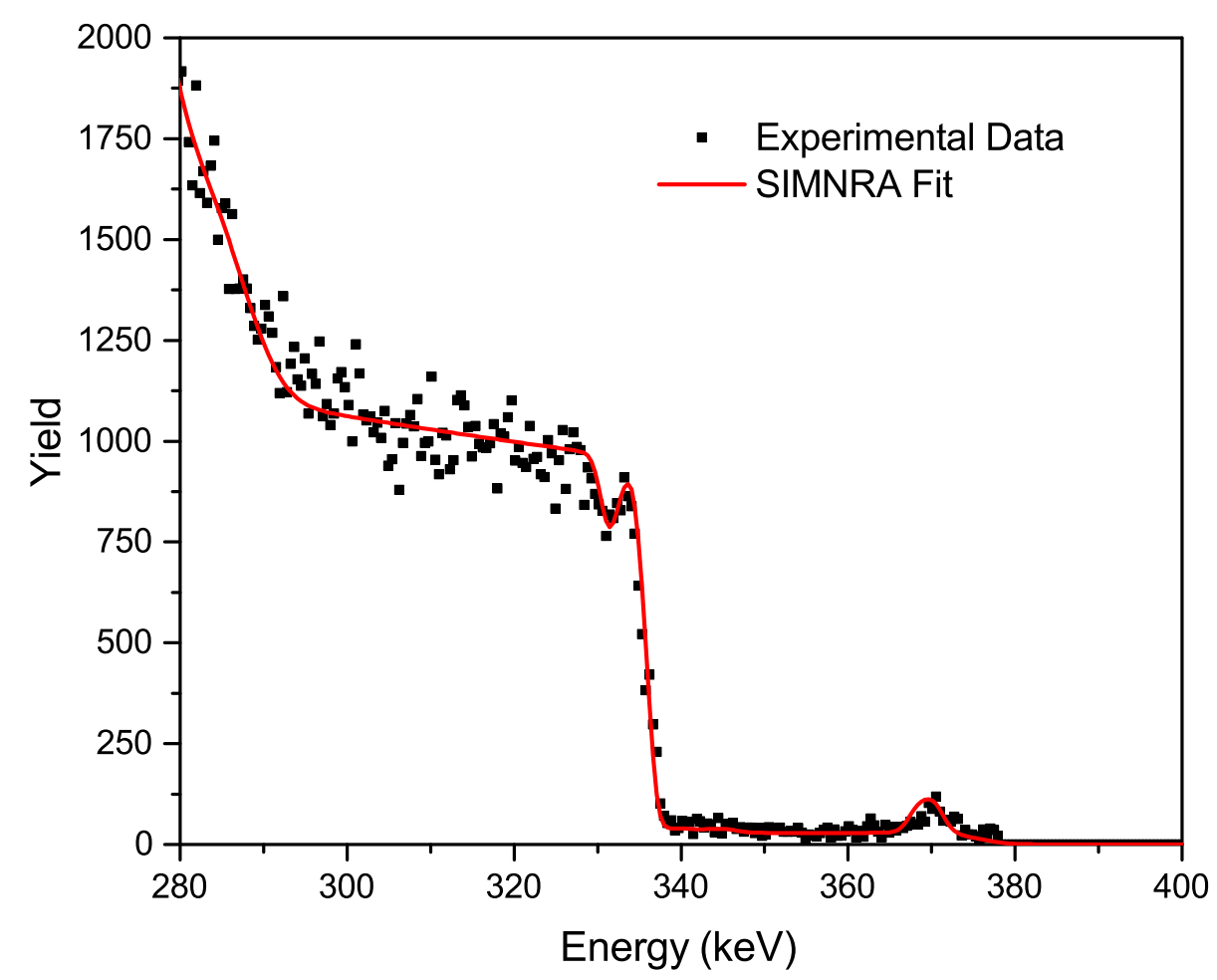

Figure 4.9: High Resolution RBS spectrum of as-deposited DLC

High-resolution RBS spectrum of as-deposited DLC synthesized by MSIBD is shown in figure 4.9. The spectrum is processed as per the steps outlined in section 3.4. The abscissa forms the energy scale after calibration of the channels by TaN standard and the 
ordinate records the number of He particles scattered from the film. The parameters for the experiment are recorded in table 4.2 .

Table 4.2: HR-RBS parameters

\begin{tabular}{|c|c|}
\hline Parameters & Values \\
\hline \hline Incident Ion & ${ }^{4} \mathrm{He}^{+}$ \\
\hline Energy & $500 \mathrm{keV}$ \\
\hline Incident Angle & $53.05^{\circ}$ \\
\hline Exit Angle & $62.37^{\circ}$ \\
\hline Scattering Angle & $65^{\circ}$ \\
\hline Calibration Offset & $277.57 \mathrm{keV}$ \\
\hline Energy per Channel & $0.4344 \mathrm{keV} / \mathrm{channel}$ \\
\hline Particles $*$ Sr & $2.626 \mathrm{E}+10$ \\
\hline Detector Resolution & $1.700 \mathrm{keV}$ \\
\hline
\end{tabular}

The dominant signal as expected arises from carbon starting from $338 \mathrm{keV}$. The carbon signal forms a plateau and stretches down below to $280 \mathrm{keV}$. This agrees with the carbon film formation discussed above. The extent to which the plateau extends denote the film thickness. The setup uses a particles*sr value of $2.1 \times 10^{10}$. The value is basically the product of the solid angle of detection and number of particles incident on the sample throughout the measurement process. As discussed in section 2.1.4, the number of scattered particles collected during the measurement is directly proportional to the solid angle of detection. Similarly the number of scattered particles detected depends directly on the number of particles incident on the sample. Thus their product is proportional to the likelihood for detection of a scattered particle at a particular energy and scattering angle. For each measurement this value may vary and this value is essential to compute the concentration of the elements composing the sample. In an RBS spectrum the height of the signal denotes their concentration. Considering the setup parameters, the height of the carbon signal corresponds to a carbon concentration of about $70-75$ at.\%. This indicates that hydrogen forms about $25-30$ at.\% of the DLC films. Another crucial information that can be gained from comparison of RBS spectrum and TEM information is the density of the film. The TEM image indicates the film to be $148 \mathrm{~nm}$ thick. RBS fitting performed by SIMNRA is shown in figure 4.9. The spectrum shows the shift of Si signal which holds information about the thickness of the film as well. RBS fit indicates the DLC film to be composed of about $1450 \times 10^{15}$ atoms. $^{-2}$. The atomic density of the film can be thus obtained from this equivalence and is $9.8 \times 10^{22}$ atoms.cm $^{-3}$

The atomic density provides information about the stopping power of the DLC films. This density corresponds to a value of $1.51 \mathrm{~g} / \mathrm{cc}$. This agrees with the range of densities $(1.2$ $-1.8 \mathrm{~g} / \mathrm{cc}$ ) recorded for hydrogenated DLC [8]. 
Analysis from Rutherford scattering provides deeper insight into the film. For example, the extent of Si dilution within DLC can be clearly extracted. In TEM the Si intermixing in the immediate vicinity of the DLC/Si interface was discussed. However, RBS analysis allows quantitative interpretation of the extent to which $\mathrm{Si}$ is diluted within DLC. The SIMNRA fit required to match the raw data indicates that a diluted $\mathrm{Si}$ concentration of about 0.4 at.\% was observed upto $40 \mathrm{~nm}$ from the surface. In other words, starting from the DLC/Si interface a minor concentration of $\mathrm{Si}$ is detected upto $100 \mathrm{~nm}$ of DLC growth. The analysis also indicates a small concentration of oxygen to be present in the surface. An additional detail obtained from the RBS spectrum is a minor dip in carbon concentration following the $\mathrm{C}$ surface edge. A possible reason for the depletion could be due to a corresponding increase in the hydrogen concentration which is explained below.

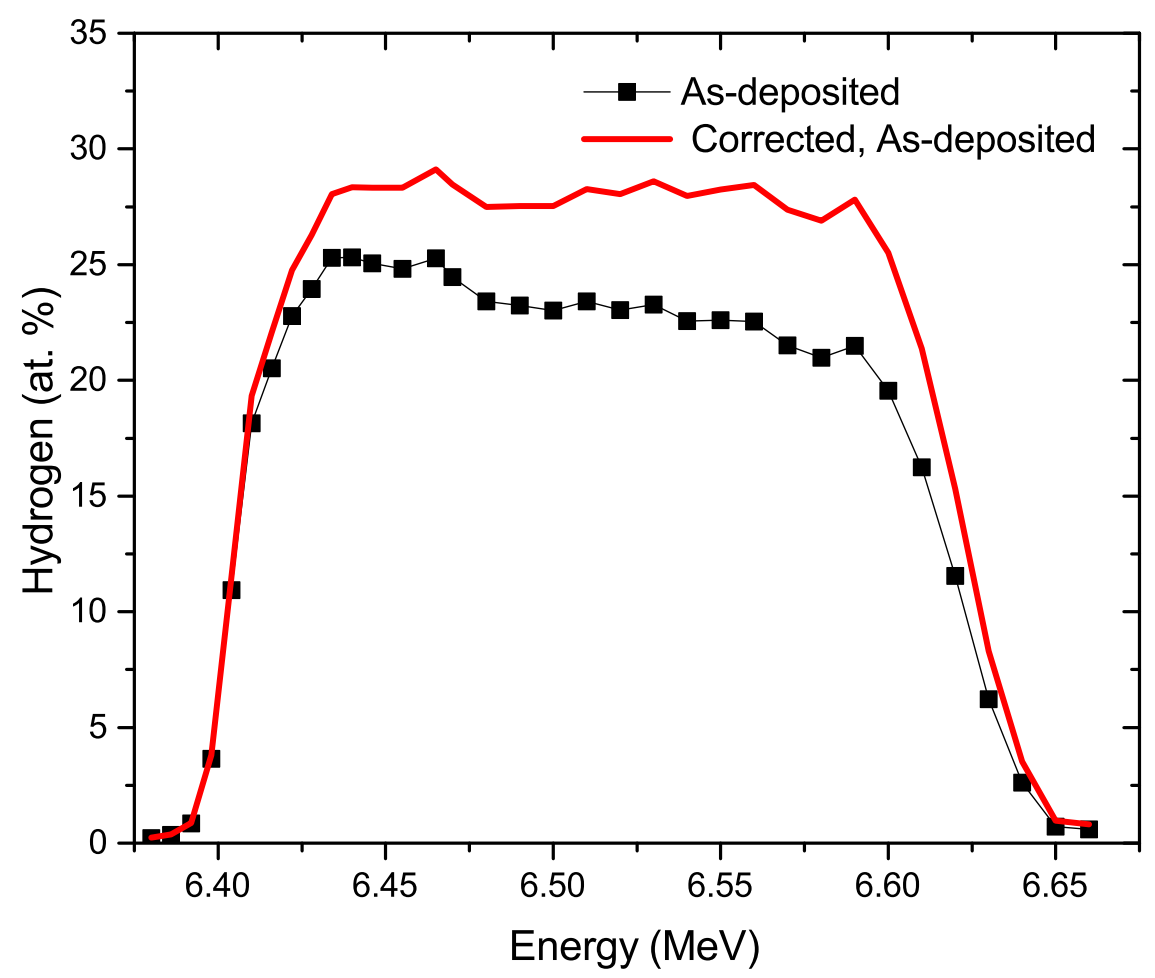

Figure 4.10: Hydrogen profile in as-deposited DLC films synthesized by MSIBD. The raw data is corrected for hydrogen loss during measurement and is overlaid on top of it. It can be seen from the corrected data that the significant decline in hydrogen concentration as function of depth arises primarily from the experimental artifact. The hydrogen concentration within the DLC films was observed to within $25-30$ at.\%.

Figure 4.10 shows the hydrogen profile of as-deposited DLC measured by RNRA [90]. 
The figure shows both the raw data and the corrected profile. The correction is required to account for the effusion of hydrogen during ion beam exposure. The effusion rate is dependent on the hydrogen bonding in the sample and it is determined to be -0.01 at. $\% \mu \mathrm{C}^{-1}$. Further details about the correction can be found in [91, 90]. The profile confirms the RBS analysis. The DLC films contain an overall hydrogen concentration of about $25-30$ at.\%. This is of interest considering the fact that the impacting molecule during the growth process contains more hydrogen (about twice) than carbon. TRIM simulations indicate that the difference in the number of hydrogen and carbon atoms sputtered during the growth process is not significant enough to explain the change in stoichiometry of $\mathrm{C}: \mathrm{H}$ from 1:2 (in molecule) to 3:1 in the DLC matrix. The measured hydrogen concentration indicates that most of the impringing hydrogen atoms forms hydrogen molecules and hence effuse out of the DLC film.

Another notable feature is the depletion of hydrogen in the surface edge marked in the figure. This depletion can have two possible reasons:

1. Graphitization: The carbon fraction relaxing during the growth of DLC accumulates towards the surface as shown in figure 4.4. Graphitic carbon do not contain dangling bonds and thus incorporate lesser hydrogen. This partially accounts for the nearsurface depletion.

2. Effusion: The other possible reason is that it is easier for the hydrogen present in the surface to capture an additional hydrogen atom which can effuse out as $\mathrm{H}_{2}$ molecule. This can happen in the deeper regions as well, however the probability of capturing an additional hydrogen atom to form $\mathrm{H}_{2}$ molecule is lower in the deeper regions and is thus difficult to effuse out during deposition.

More details about the near-surface depletion can be found from our work [91]. There is also a minor increase in the hydrogen concentration immediately following the surface edge. This can possibly explain the minor dip in carbon concentration observed in RBS spectrum.

Raman spectroscopy is a widely used method to probe the structure of diamond-like carbon films $[92,10]$. The Raman spectrum of single crystalline graphite shows a sharp peak at $1580 \mathrm{~cm}^{-1}$. This peak is denoted as the graphite peak or G peak. The G peak arises from the stretching mode of the $\mathrm{sp}^{2}$ bonded carbon atoms. For diamond a single sharp peak is observed at $1392 \mathrm{~cm}^{-1}$. The Raman spectra for DLC is a combination of both these phases, however in visible Raman spectroscopy the vibrational modes of $\mathrm{sp}^{2}$ bonded carbon dominate the spectra since the cross section of the $\mathrm{sp}^{2}$ phase is much higher than their $\mathrm{sp}^{3}$ counterparts $[92,10]$. Apart from the $\mathrm{G}$ peak, an additional peak around $1360 \mathrm{~cm}^{-1}$ is observed for DLC films. This peak is referred to as D peak or disorder peak and is said to originate from the breathing modes of six-fold carbon rings located near the defects or 
dislocations within the films. The intensity, width and position of these peaks are used to extract the structural details of the films. For example, the ratio of the integrated intensity of the $D$ and $G$ peaks, $I(D) / I(G)$, reflects the contribution from the $\mathrm{sp}^{2}$ clusters within the film. The $\mathrm{G}$ peak position is usually associated with the $\mathrm{sp}^{2}$ content and disorder of the films. The $\mathrm{G}$ peak position also depends on the excitation energy. For carbon films containing purely sp $\mathrm{sp}^{2}$ bonded carbon, the $\mathrm{G}$ peak saturates at $1600 \mathrm{~cm}^{-1}$, however for DLC films the G peak can rise beyond $1600 \mathrm{~cm}^{-1}$ with increasing energy mainly due to the increased disorder present in the films. The FWHM of the G peak on the other hand is a direct reflection of the disorder present in the films. A higher FWHM indicates higher disorder reflecting an increase in density, strength and hardness of the films. Figure 4.11 shows the Raman spectrum obtained from the as-deposited DLC film by MSIBD.

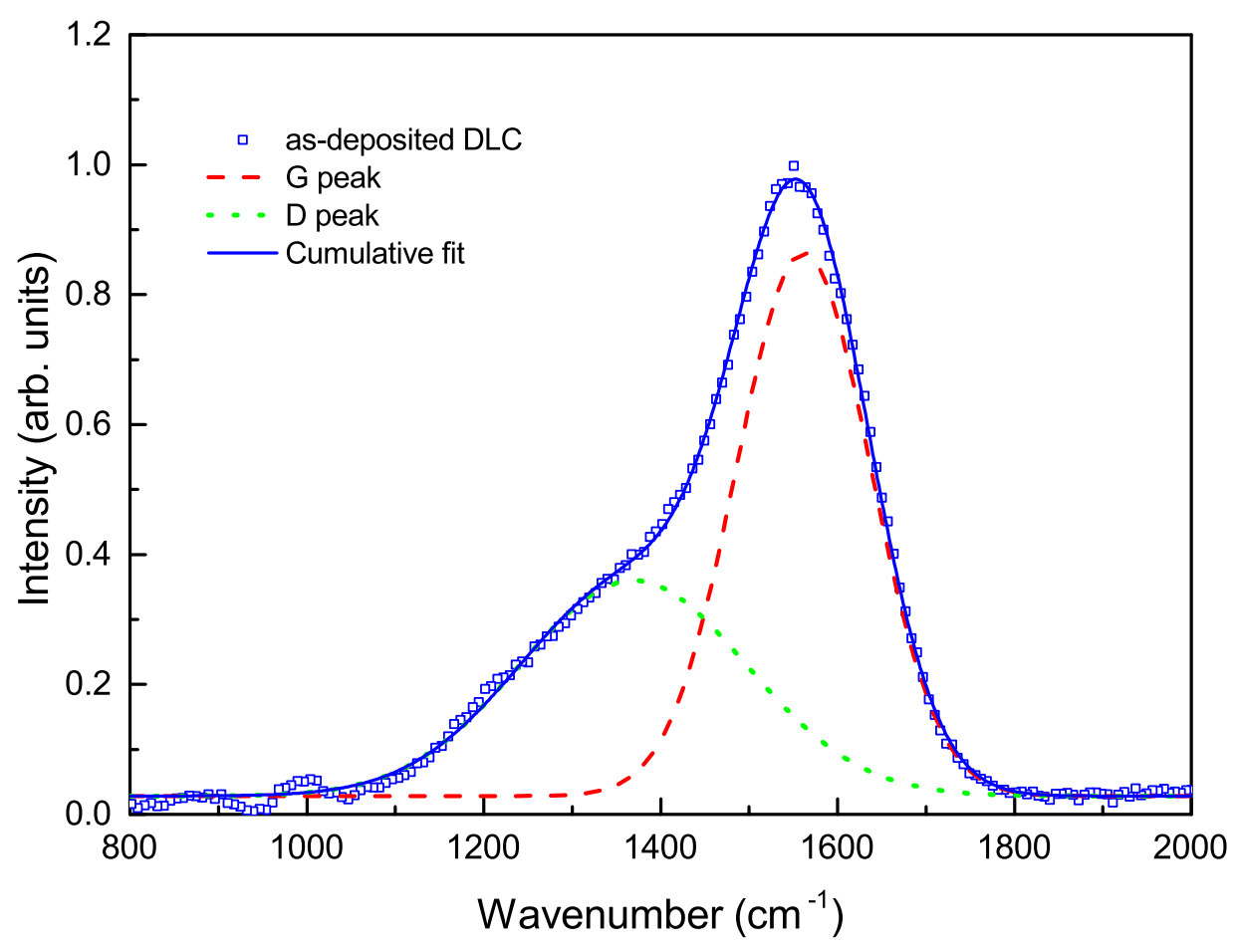

Figure 4.11: Raman spectrum of as-deposited DLC films synthesized by MSIBD. The data is fit with two Gaussian peaks representing the D (green) and G (red) peak whose sum fits the measured data well.

The Raman spectra of as-deposited DLC is fit with a double Gaussian fit. The Gaussian centered at 1558 represents the $\mathrm{G}$ peak and the peak centered around 1360 represents the D peak. The $\mathrm{I}(\mathrm{D}) / \mathrm{I}(\mathrm{G})$ ratio computed for the above film is 0.55 and the full width half- 
maximum of $\mathrm{G}$ peak from the fit is $178 \mathrm{~cm}^{-1}$. The widely accepted three stage model proposed by Robertson and Ferrari [24] was used to identify the disorder and corresponding $\mathrm{sp}^{3}$ content of the films. The model describes the transition of carbon from pure graphite $\left(100 \% \mathrm{sp}^{2}\right.$ content) to tetrahedral amorphous DLC films $\left(\mathrm{sp}^{3}\right.$ content $\left.>85 \%\right)$ in three stages. The model relates the $\mathrm{G}$ peak position and $\mathrm{I}(\mathrm{D}) / \mathrm{I}(\mathrm{G})$ ratio to the disorder, $\mathrm{sp}^{3}$ content and $\mathrm{sp}^{2}$ configuration of the film. For the as-deposited films, the $G$ peak position and $\mathrm{I}(\mathrm{D}) / \mathrm{I}(\mathrm{G})$ ratio points to an $\mathrm{sp}^{3}$ content greater than $30 \%$ [93].

\subsection{Direct Ion beam deposition}

Diamond-like carbon films were also synthesized by direct ion beam deposition. The prime difference between MSIBD and DIBD is the mass-selection of ion species. Selection of ion species not only controls the $\mathrm{C}: \mathrm{H}$ stoichiometry of the molecular ion beam, it also allows precise control over the energy of deposition. In the case of DIBD, all positive ions produced from the butane plasma are extracted towards the sample stage. The details about the different species making up the ion beam and their respective abundance can be found in table 4.3 .

The films are deposited by the system described in section 3.2. The usual operating conditions involve usage of a butane precursor gas to supply a carbon rich plasma. The butane is supplied through a gas feed connected to the Penning gas ion source. The base pressure of the ion source before the introduction of the gas is generally $8 \times 10^{-7} \mathrm{hPa}$. Tantalum targets are employed as the metallic cathodes and the hollow anode is made of aluminum. Typical anode voltage applied under normal operating conditions is $2 \mathrm{kV}$. The magnetic field needed for the plasma generation is provided by the solenoid which is usually operated with a current of $\sim 1.6-1.8 \mathrm{~A}$. The operating pressure of the ion source is generally in the range of $\sim 2 \times 10^{-5} \mathrm{hPa}$ near the ion beam exit. $3 \mathrm{kV}$ negative bias voltage is applied to the sample stage to accelerate the ion beam. Thus the ions experience an overall acceleration voltage of $5 \mathrm{kV}$. Since the ion beam already covers a significant portion of the sample stage, scanners are usually not employed. The typical ion current during DLC deposition is about $40 \mu \mathrm{A}$. Each deposition run covers a substrate area of about $4 \mathrm{~cm}^{2}$.

Ion beam extracted from the Penning ion source with the use of butane as the precursor gas can result in a wide range of hydrocarbon beams. The different ions making up the ion beam and their relative composition are given in table 4.3 . 
Table 4.3: Ion species produced by the Penning ion source operating with butane gas

\begin{tabular}{|c|c|c|}
\hline Ion species & Relative percentage & Ion species with highest probability \\
\hline \hline $\mathrm{H}_{x}^{+}$ & $17.6 \pm 0.9$ & $\mathrm{H}_{2}^{+}$ \\
\hline $\mathrm{CH}_{x}^{+}$ & $21.9 \pm 1.1$ & $\mathrm{CH}_{4}^{+}$ \\
\hline $\mathrm{C}_{2} \mathrm{H}_{x}^{+}$ & $41.1 \pm 2.1$ & $\mathrm{C}_{2} \mathrm{H}_{4}^{+}$ \\
\hline $\mathrm{C}_{3} \mathrm{H}_{x}^{+}$ & $17.6 \pm 0.9$ & $\mathrm{C}_{3} \mathrm{H}_{6}^{+}$ \\
\hline $\mathrm{C}_{4} \mathrm{H}_{y}^{+}$ & $0.8 \pm 0.1$ & $\mathrm{C}_{4} \mathrm{H}_{3}^{+}$ \\
\hline
\end{tabular}

It can be seen that molecular ions ranging from $\mathrm{C}_{4} \mathrm{H}_{y}^{+}$and $\mathrm{C}_{3} \mathrm{H}_{x}^{+}$to elemental ions such as $\mathrm{H}$ and $\mathrm{C}$ are present in the ion beam. A relatively low concentration of multiply charged ions can also be generated from the ion source. While all the ions are accelerated by the same potential of $5 \mathrm{kV}$, the energy of the ions upon impact onto the substrate differs greatly. Molecular ion beams upon impact split into elementary ions. The energy of the molecular ion is distributed among the elementary ions based on their mass. $\mathrm{A}_{3} \mathrm{H}_{x}^{+}$molecule upon impact splits into $3 \mathrm{C}$ atoms with $1429 \mathrm{eV}$ each and $6 \mathrm{H}$ atoms with $119 \mathrm{eV}$ each. Since the ion beam is made up of multiple species, the energy of carbon atoms can range from 10 $\mathrm{keV}$ to $1 \mathrm{keV}$ and $\mathrm{H}$ can range from $5 \mathrm{keV}$ to $120 \mathrm{eV}$. The primary difference associated with such differences in the ion energy is the penetration depth of the ions. The film growth in this case happens simultaneously at different regions within the DLC film. The film was also shown to incorporate higher hydrogen content possibly due to the varied concentration of $\mathrm{C}$ and $\mathrm{H}$ impacting the substrate [3].

The films are generally grown to a thickness of $500 \mathrm{~nm}$ on a Si substrate which takes about 8 hours machine time. The substrate is cleaned by pressurized air before deposition. No other chemical or physical treatment are performed on the Si substrate prior to deposition. The incident ion beam anyway sputters away most of the impurities present on top of the Si substrate. Since scanning is not applied the thickness of the film is highest at the center and falls off gradually towards the edges. Application of these films for ion implantation demands a minimum thickness of $60 \mathrm{~nm}$ (calculated based on the range of Co ions) and there is no upper limit placed on the thickness of the samples. The films satisfy the criteria. 


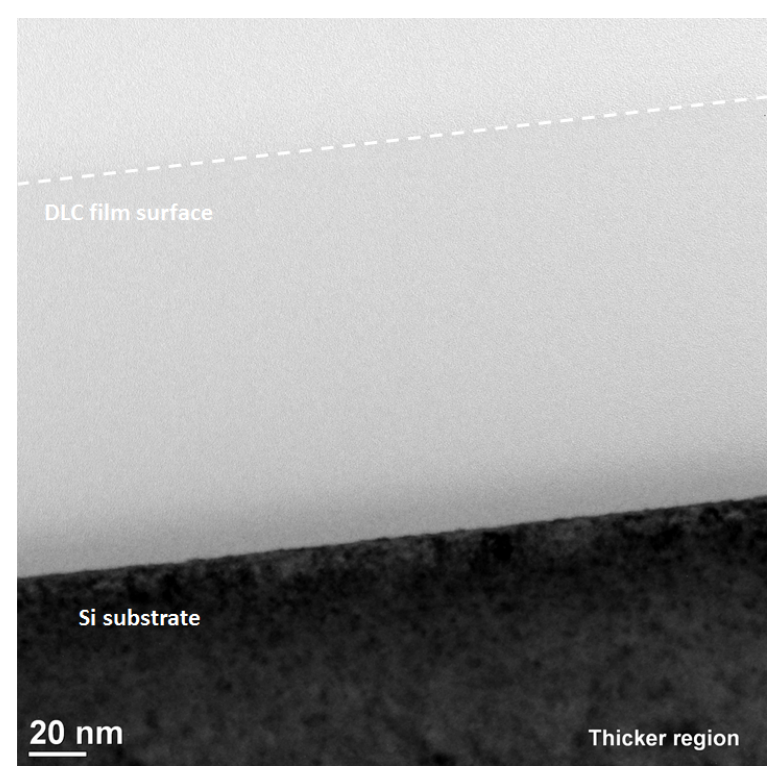

Figure 4.12: Cross-sectional TEM image of as-deposited DLC synthesized by DIBD

Figure 4.12 shows the cross-sectional TEM image of DLC deposited by DIBD at $4.5 \mathrm{keV}$ energy. Even though the deposition voltage is slightly lower than the MSIBD film, it can be seen that the extent of $\mathrm{C}$ and $\mathrm{Si}$ intermixing is higher in this case due to the increased penetration range of a fraction of carbon ions. The film is again completely amorphous and uniform through out the sample. For the purpose of this research, implantation was performed on DIBD films to verify if similar structural effects manifest in these films as observed for MSIBD films. DIBD method also have several practical advantages. More samples can be deposited in limited time due to higher beam coverage and greater deposition rates allowed by the method and the experimental setup. More information about DIBD films can be found in [3].

\subsection{Effect of Ion Energy}

In the case of ion beam deposition of DLC the most crucial parameter is the energy of deposition. Increasing the ion energy has a detrimental effect on the $\mathrm{sp}^{3}$ content of the DLC films [8]. However, the literature so far records the value only at low deposition energies $(<$ $2 \mathrm{keV}$ ). The primary reason for carrying out DLC deposition at higher energies is to obtain a faster film growth which is identified as one of the key disadvantage of using ion beam deposition for DLC growth. 


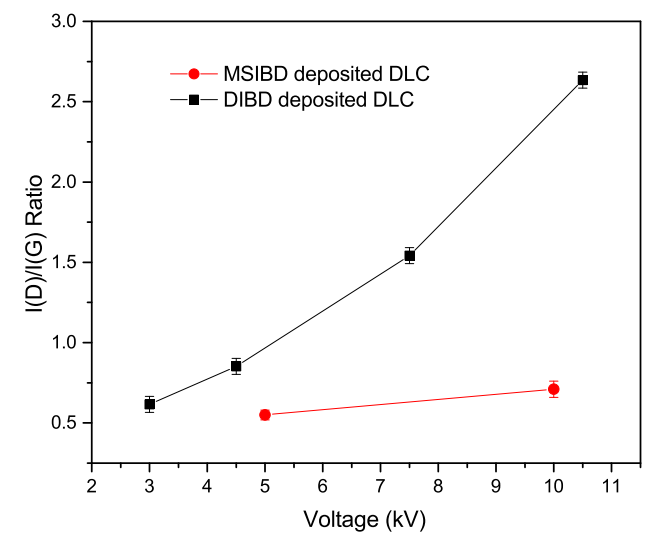

(a) $\mathrm{I}(\mathrm{D}) / \mathrm{I}(\mathrm{G})$ ratio of DIBD and MSIBD deposited DLC as a function of acceleration voltage

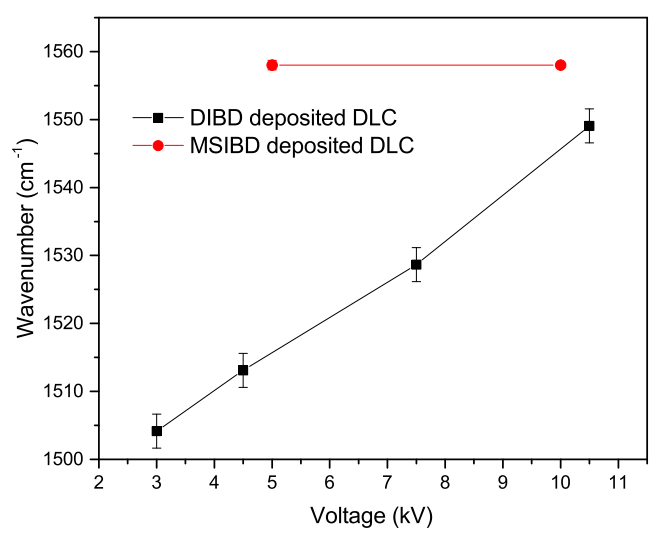

(b) G peak position of DIBD and MSIBD deposited DLC as a function of acceleration voltage

Figure 4.13: Raman analysis of DIBD and MSIBD deposited DLC as a function of acceleration voltage. In the case of DIBD films it can be seen that the $\mathrm{I}(\mathrm{D}) / \mathrm{I}(\mathrm{G})$ ratio and $\mathrm{G}$ peak position shows significant increase with acceleration voltage, whereas for MSIBD film the changes are not significant. This indicates that for DIBD, increase in acceleration voltage results in increase in overall $\mathrm{sp}^{2}$ concentration. While for MSIBD, increase in acceleration voltage increases the size of $\mathrm{sp}^{2}$ clusters.

Figure 4.13 depicts the Raman analysis of DLC films deposited at different energies by both DIBD deposition and MSIBD deposition $\left(\mathrm{C}_{3} \mathrm{H}_{6}^{+}\right)$. As mentioned earlier the $\mathrm{sp}^{2}$ contribution in the film can be ascertained from the $\mathrm{I}(\mathrm{D}) / \mathrm{I}(\mathrm{G})$ ratio and the $\mathrm{G}$ peak position. The three stage model proposed by Ferrari and Robertson can be explained from the figure [10] below. 


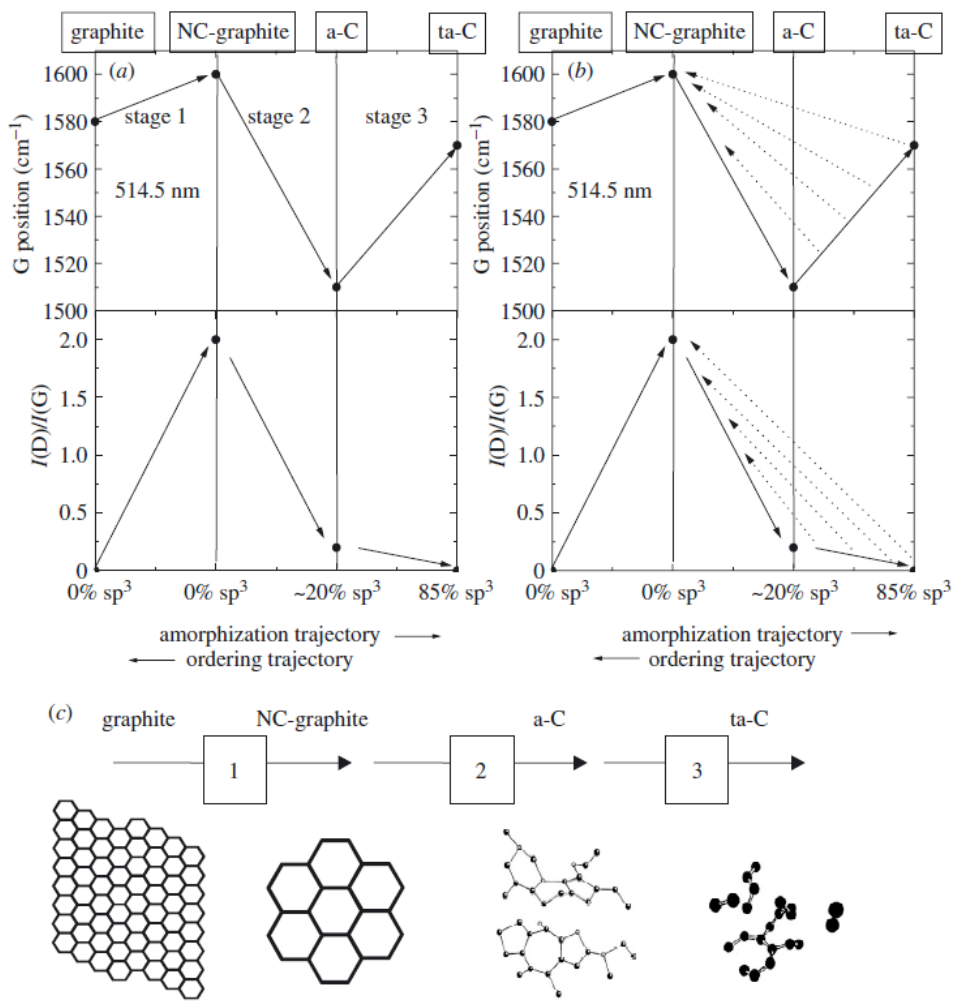

Figure 4.14: Three stage model proposed by Ferrari and Robertson describing the transition of carbon from crystalline graphite to tetrahedral amorphous carbon. The model plots the trend taken by two measured parameters from Raman spectrum, the $\mathrm{I}(\mathrm{D}) / \mathrm{I}(\mathrm{G})$ ratio and G peak position (shown in (a) and (b)), as the carbon transforms from graphite to nanocrystalline graphite then further to amorphous carbon and ultimately to tetrahedral amorphous carbon (as shown in (c)). The stage of the as-deposited and implanted DLC films in this work can be identified by comparing their Raman parameters $(\mathrm{I}(\mathrm{D}) / \mathrm{I}(\mathrm{G})$ ratio and $\mathrm{G}$ peak position) with the three-stage model. Source [10]

The figure shows the transition in $\mathrm{I}(\mathrm{D}) / \mathrm{I}(\mathrm{G})$ ratio and $\mathrm{G}$ peak position as the carbon transforms from

1. Crystalline graphite to graphite nanoclusters

2. Graphite nanoclusters to amorphous carbon

3. Amorphous carbon to tetrahedral $\mathrm{sp}^{3}$ carbon

It can be seen that depending on the stage at which the carbon films are present (from the above four), an increase in $\mathrm{I}(\mathrm{D}) / \mathrm{I}(\mathrm{G})$ ratio can mean both an increase or decrease in $\mathrm{sp}^{2}$ character. However, considering the amorphous nature of the film from TEM it can be confirmed that the film is already in the amorphous stage. Hence an increase in $\mathrm{I}(\mathrm{D}) / \mathrm{I}(\mathrm{G})$ 
ratio indicates a shift of the amorphous carbon towards graphite nanoclusters thereby increasing the $\mathrm{sp}^{2}$ content. This contribution can arise either from hybridization of $\mathrm{sp}^{3}$ to $\mathrm{sp}^{2}$ carbon or from the growth of $\mathrm{sp}^{2}$ clusters. If the increase in $\mathrm{sp}^{2}$ character of the film is from hybridization, then a change in $\mathrm{I}(\mathrm{D}) / \mathrm{I}(\mathrm{G})$ ratio should record a simultaneous shift in $\mathrm{G}$ peak position. This is observed in the case of DIBD films. On the other hand a similar shift in position is not observed in the case of MSIBD films, This indicates that the $\mathrm{sp}^{2}$ character in the case of MSIBD films are due to increase in size of sp $^{2}$ clusters [93].

A similar work carried out on DIBD deposited films at GNS Science shows the $\mathrm{sp}^{3}$ content of the films to range from $40-50 \%$ using X-ray analysis [91]. This confirms the above analysis that the (a) the $\mathrm{sp}^{3}$ content of the film is above $30 \%$ and (b) increasing ion energy increases the $\mathrm{sp}^{2}$ character of the film.

\subsection{Conclusion}

This chapter covered the synthesis of DLC films by ion beam deposition. The chapter began with a brief introduction and classification of DLC films. Various growth mechanisms proposed for growth of DLC was discussed and the key factors responsible for formation of $\mathrm{sp}^{3}$ phase was identified. DLC films used in this work were deposited by MSIBD and DIBD processes. The parameters used for synthesis were presented followed by discussion on the properties of as-deposited films. DLC films used for Co implantation were synthesized with $\mathrm{C}_{3} \mathrm{H}_{6}^{+}$ions with $5 \mathrm{keV}$ deposition energy. The films were deposited typically to a thickness of $100 \mathrm{~nm}$ with 25 - 30 at.\% hydrogen content. Raman spectroscopy revealed that the films contain at least $30 \% \mathrm{sp}^{3}$ content. Finally, the chapter was concluded with a discussion on the role of deposition energy on the property of the deposited films. It was found out that increase in ion energy results in decrease in the $\mathrm{sp}^{3}$ character of the DLC films. 



\section{Chapter 5}

\section{Structure and Distribution}

\subsection{Introduction}

This chapter deals with low energy Co implantation into DLC films. The previous chapter covered the synthesis of diamond-like carbon films by high energy ion beam deposition. In this chapter, the focus is on structural analysis of Co implanted DLC films. The chapter begins with the results predicted from ballistics of Co implantation into DLC. DTRIM, a Monte-Carlo simulation code is used to compute the distribution of deposited energy, accumulated damage and the elemental distribution of different atoms making up the implanted films. The predicted distribution act as a base against which the implantation profile measured from HR-RBS can be compared. Analysis of HR-RBS measurements provides crucial information regarding the various effects of ion implantation in DLC. This is followed by TEM analysis of the sample. TEM measurements compliment the HR-RBS measurements and reveal several important features of the implanted samples. Finally, a model is proposed to explain the unique findings of this research.

\subsection{Ion Implantation}

In this section, the details of the ion implantation carried out on DLC films are presented. As mentioned earlier, the DLC films are implanted with Co at different fluences. The implantation energy was however, kept constant to enable comparison between different implanted films. The important implantation parameters are shown in table 5.1. 
Table 5.1: Ion implantation parameters

\begin{tabular}{|c|c|}
\hline Parameters & Value \\
\hline \hline Anode Voltage & $2 \mathrm{kV}$ \\
\hline Anode Current & $20 \mathrm{~mA}$ \\
\hline Solenoid Current & $2 \mathrm{~A}$ \\
\hline Total acceleration Voltage & $30 \mathrm{kV}$ \\
\hline Einzellens Voltage & $27 \mathrm{kV}$ \\
\hline Mass separation field & $4566 \mathrm{G}$ \\
\hline Unscanned Ion current & $10 \mu \mathrm{A} . \mathrm{cm}^{-2}$ \\
\hline Scanned Ion current & $5 \mu \mathrm{A} . \mathrm{cm}^{-2}$ \\
\hline Electron suppression & $400 \mathrm{~V}$ \\
\hline
\end{tabular}

Different samples implanted with Co and their fluences are tabulated in table 5.1 below.

Table 5.2: Details of implantation fluence for samples used in this chapter

\begin{tabular}{|c|c|c|c|c|}
\hline S.No & DLC & Ion & Fluence $\left(\times 10^{16}\right.$ atoms.cm $\left.^{-2}\right)$ & Sample Code \\
\hline 1 & a:C-H & Co $^{+}$ & 1.2 & Co 1.2 \\
\hline 2 & a:C-H & $\mathrm{Co}^{+}$ & 2.4 & Co 2.4 \\
\hline 3 & a:C-H & $\mathrm{Co}^{+}$ & 4 & Co 4 \\
\hline 4 & a:C-H & $\mathrm{Co}^{+}$ & 7 & Co 7 \\
\hline 5 & a:C-H & $\mathrm{Co}^{+}$ & 12 & Co 12 \\
\hline
\end{tabular}

\subsection{Simulations}

Ion implantation simulations can predict the implantation and energy deposition profiles based on the input parameters. DTRIM is one such implantation simulation software which has been used in this research, to predict the elemental distribution in implanted DLC. The main input parameters needed for this simulation are ion energy, atomic number, mass, fluence, target composition and density. For cobalt implantation into DLC the ion is $\mathrm{Co}^{+}$and the target is DLC. Cobalt's atomic number is 27 and mass is 58.93 a.m.u. The composition and density of DLC were determined in the previous chapter. DLC is composed of carbon and hydrogen with a stoichiometry of 3:1 respectively. DLC density was determined to be $1.51 \mathrm{~g} / \mathrm{cc}$. The last remaining parameters needed for the simulation are ion energy and fluence. 
Considering the wider perspective of this research, it is more beneficial to study the change in DLC properties as a function of ion fluence rather than ion energy. Changing the ion fluence reflects directly on the concentration of Co incorporated into DLC. On the other hand variation in ion energy modifies mainly the depth and distribution of Co in DLC. There are several limitations associated with such changes. For example, if the selected energy is not high enough, following effects can be observed at relatively lower fluences,

- accumulation of implanted ions at the surface leading to oxidation effects

- significant loss of implanted ions due to sputtering effects

- achieving steady-state condition

However, if the energy selected is very high,

- distribution profile becomes broader requiring very high fluences to achieve the required concentration density

- the changes induced by ion implantation will not be concentrated in the near-surface region

The last factor in particular plays a crucial role when employing surface sensitive techniques (eg., electrical resistance measurements) to characterize the films. Considering the above limitations and the practical constraints, the implantation energy was fixed to be $30 \mathrm{keV}$. The implantation fluence was thus the only parameter varied. Implantation fluence ranging from $0.8-20 \times 10^{16}$ atoms.cm ${ }^{-2}$ were experimented both in the simulations and in the experiment. In the following sections, some important information gathered from DTRIM simulations based on the said parameters and range of implantation fluence are presented. 


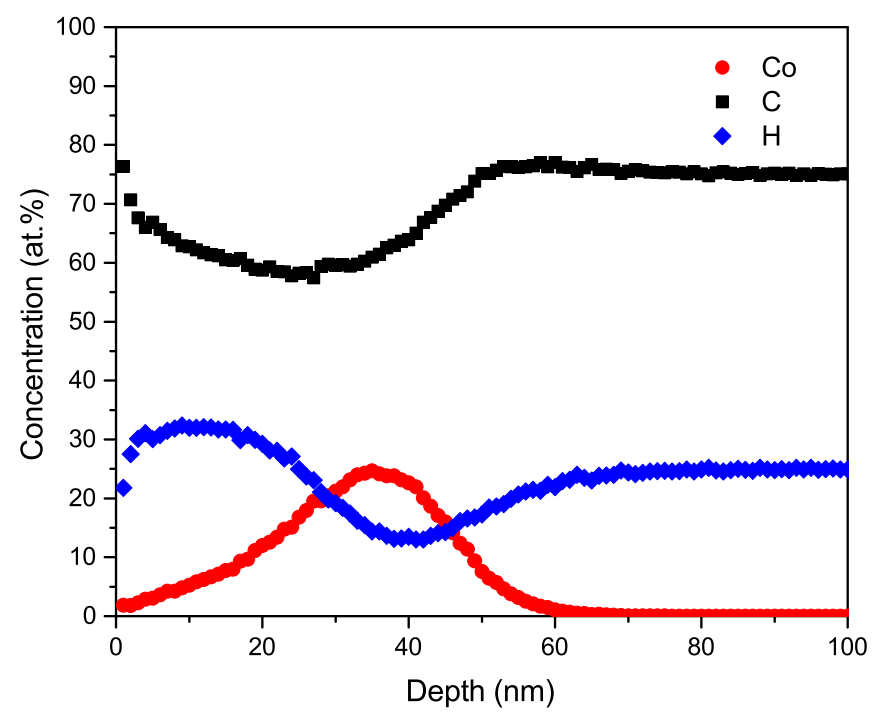

(a) Elemental distribution of Co implanted DLC

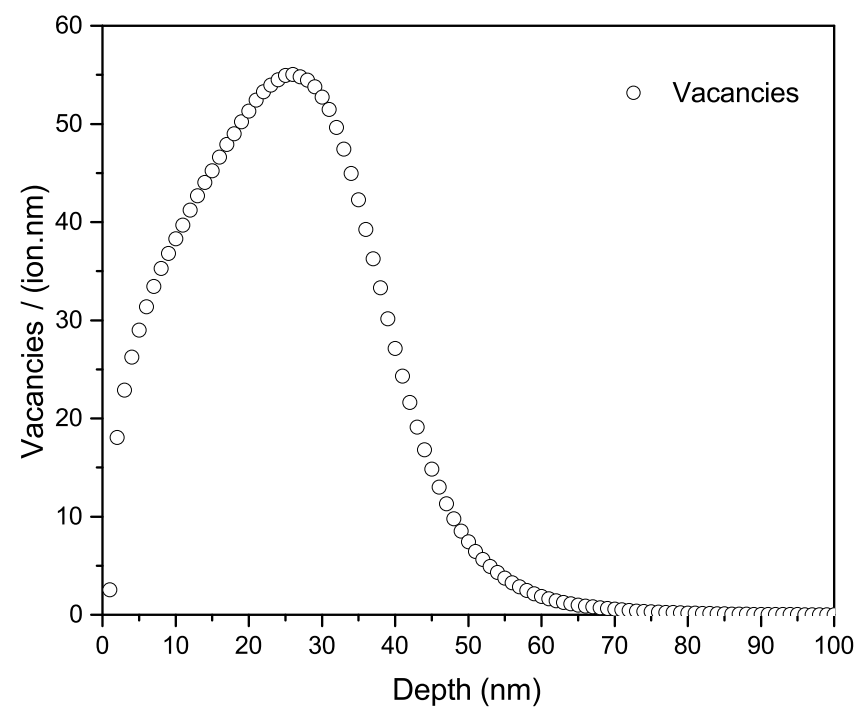

(b) Vacancy distribution of Co implanted DLC

Figure 5.1: Theoretical predication of elemental and vacancy distribution in Co - DLC films at an implantation fluence of $\mathrm{F}=7 \times 10^{16}$ atoms.cm ${ }^{-2}$ by DTRIM.

Figure 5.1 shows the elemental and vacancy distribution of Co implanted into DLC for a fluence of $7 \times 10^{16}$ atoms.cm ${ }^{-2}$. This is the most studied sample in this research. As shown in the figure 5.1 (a), DTRIM predicts Co to assume nearly a Gaussian distribution with a mean projected range of $33 \mathrm{~nm}$ and a straggling of $11.5 \mathrm{~nm}$. Co assumes a peak concentration of 24.7 at.\%. A small fraction of Co (1.8 at.\%) reaches the target surface. The figure also 
shows the $\mathrm{C}$ and $\mathrm{H}$ profile of the implanted DLC film predicted from DTRIM simulation. The $\mathrm{C}$ profile corresponds inversely to that of the Co profile. Carbon concentration gradually decreases from surface and reaches its minima of 57 at.\% just before the Co peak. Beyond the projected range, $\mathrm{C}$ concentration gradually increases again finally reaching a maximum concentration of 77 at.\%. C profile finally stabilizes at of 75 at.\% which is also its original concentration before implantation. This region denotes the end of range for Co.

Hydrogen interestingly shows a profile different to that of carbon. Hydrogen being highly mobile can rearrange to greater distance than that of Co or C. Also, effects of forward recoil collisions and recoil implantation leads to accumulation of hydrogen in the surface region. Additionally the sputtering yield of $\mathrm{H}$ is lower than that of $\mathrm{C}$ leading to greater $\mathrm{H}$ concentration at the near surface region. The only similarity shared by $\mathrm{C}$ and $\mathrm{H}$ profile is that their minimum concentration are very close to Co peak which is expected.

Apart from the elemental distribution, DTRIM also computes the damage induced in the DLC films including the effects of defect generation and sputtering. Figure 5.1 (b) shows the vacancy distribution generated by Co implantation. The vacancy peak can be observed at a depth of $24 \mathrm{~nm}$ just before the $R_{\mathrm{p}}$. This region is predicted to accumulate the maximum damage generated during implantation. DTRIM further predicts the effects of sputtering as well. For a fluence of $7 \times 10^{16}$ atoms.cm ${ }^{-2}$, about $7 \mathrm{~nm}$ of DLC is expected to be sputtered by the end of implantation. Fortunately, DTRIM takes into account the effect of sputtering while computing the elemental distribution profile. In the below sections, effects of varying fluence, ions and base matrix are covered.

\subsubsection{Ion fluence}

A wide range of implantation fluence were experimented. Figure 5.2 shows the variation in Co distribution as the fluence is varied from 1.2 to $20 \times 10^{16}$ atoms.cm ${ }^{-2}$. 


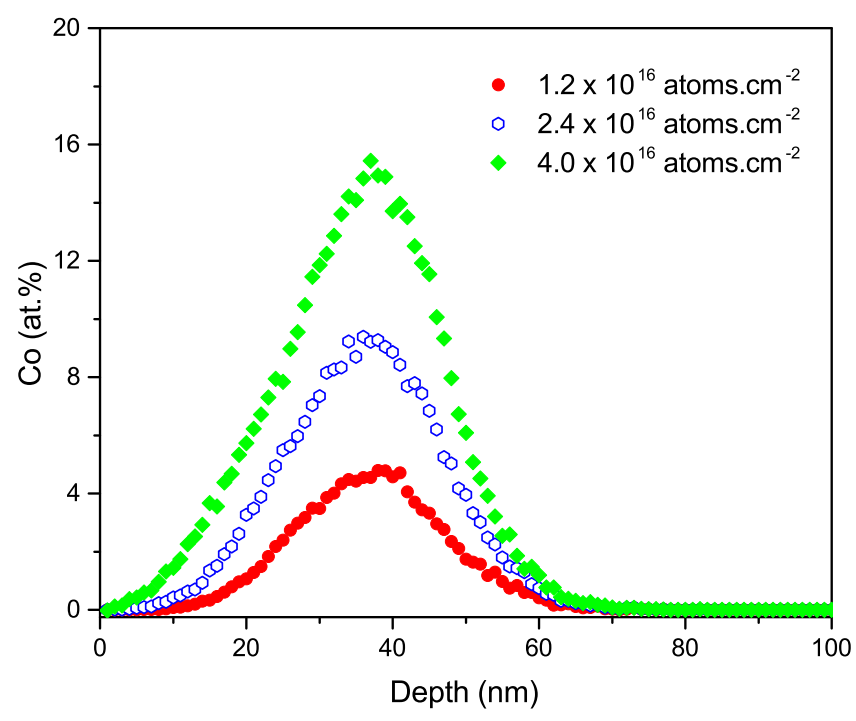

(a) Co distribution at $1.2-4 \times 10^{16}$ atoms.cm ${ }^{-2}$.

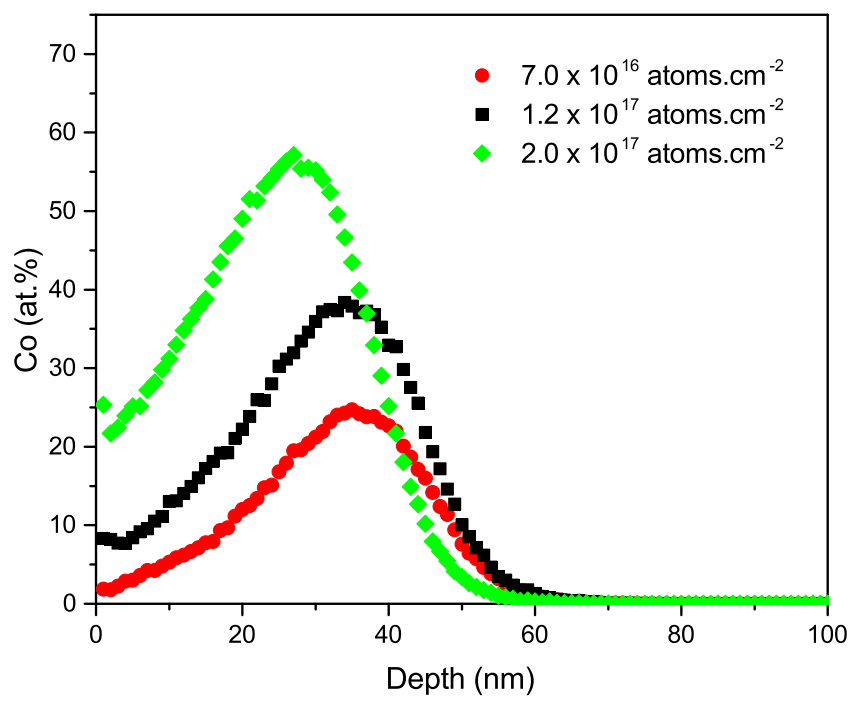

(b) Co distribution at 7- $20 \times 10^{16}$ atoms.cm ${ }^{-2}$

Figure 5.2: Predicted Co distribution in DLC at different implantation fluences by DTRIM. As the fluence increases it can be seen that the peak and surface concentration of Co increases and the peak position moves closer towards the surface.

Figure 5.2 (a) depicts Co distributions at fluence ranging from $1.2-4 \times 10^{16}$ atoms.cm $^{-2}$ (lower range) and figure 5.2 (b) shows Co distributions for implantation fluence ranging from 7 - $20 \times 10^{16}$ atoms.cm ${ }^{-2}$ (upper range). Co distributions obtained from simulations were fit with a Gaussian distribution. The details from the fit and the output files of simulations are 
shown in table 5.3.

There are clear differences between these two set of distributions. At lower fluences, Co distributions show better fit with Gaussian distribution than at higher fluence. As the fluence increases the distribution tends to be asymmetric with greater accumulation of Co before the mean projected range. This can be attributed to the increased stopping observed by the incoming Co ions as the Co concentration in the DLC increases. This effectively reduces the mean projected range as shown in table 5.3, thereby increasing the amount of Co accumulated before the $R_{p}$ compared to depths greater than $R_{p}$.

Another effect that is observed at higher fluences, is the accumulation of Co at surface. The surface concentration of Co which is 0 at.\% upto $4 \times 10^{16}$ atoms.cm $^{-2}$ increases to 21.6 at. $\%$ at $20 \times 10^{16}$ atoms.cm ${ }^{-2}$. This can be attributed to the width of the distribution. At lower range, the width of distribution is small enough to be confined in the near surface region without Co reaching the surface. However, as Co fluence increases, the width of distribution increases. Along with the increasing stopping experienced by the Co ions at higher fluences, the distribution of Co start reaching the surface.

Since the width of Co distribution increases with implantation fluence, it would be reasonable to expect Co to reach greater depths in the DLC films. While this is true at lower fluences, this does not seem to be the case at higher fluence. For example, at a depth of 50 $\mathrm{nm}$, Co implanted to a fluence of $7 \times 10^{16}$ atoms. $\mathrm{cm}^{-2}$ is predicted to result in a Co concentration of 6 at.\%. When the fluence is increased to nearly three times its original value, i.e., at a fluence of $20 \times 10^{16}$ atoms.cm ${ }^{-2}$ surprisingly the Co concentration at the same depth (50 nm) reduces to $\sim 3$ at.\%. This seemingly contradictory prediction can be explained by considering the effect of sputtering. Bombardment of Co ions on to the surface causes the sputtering of atoms from the top layer of the target matrix. At a fluence of $7 \times 10^{16}$ atoms.cm ${ }^{-2}$, the sputtering is predicted to etch away 7 nm's of DLC film. At $20 \times 10^{16}$ atoms.cm $^{-2}$, the sputtered layer amounts to $27 \mathrm{~nm}$. This explains the shift in the end of range distribution of Co atoms and observation of high Co concentration at surface for the highest Co fluence.

\subsubsection{Sputtering}

The final effect that is to be detailed before concluding the section on simulation is sputtering. The effect of sputtering was briefly discussed when comparing the Co distribution at different fluences. More detailed information about sputtering is shown in table 5.4 below,

It can be seen from the table that the effects of sputtering are more significant at higher fluences. Roughly for every $1 \times 10^{16}$ atoms implanted into $1 \mathrm{~cm}^{2}$ area of DLC at least $1 \mathrm{~nm}$ of DLC is sputtered out. Another interesting feature to be observed is the changes in the overall sputter yield of the DLC film. Initially, as the fluence increase the sputter yield seems 


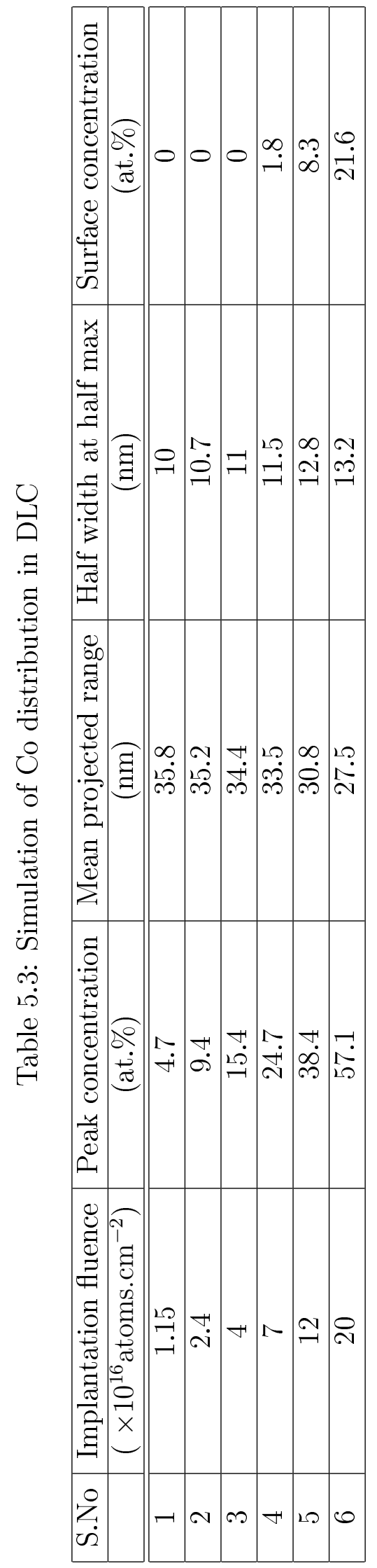




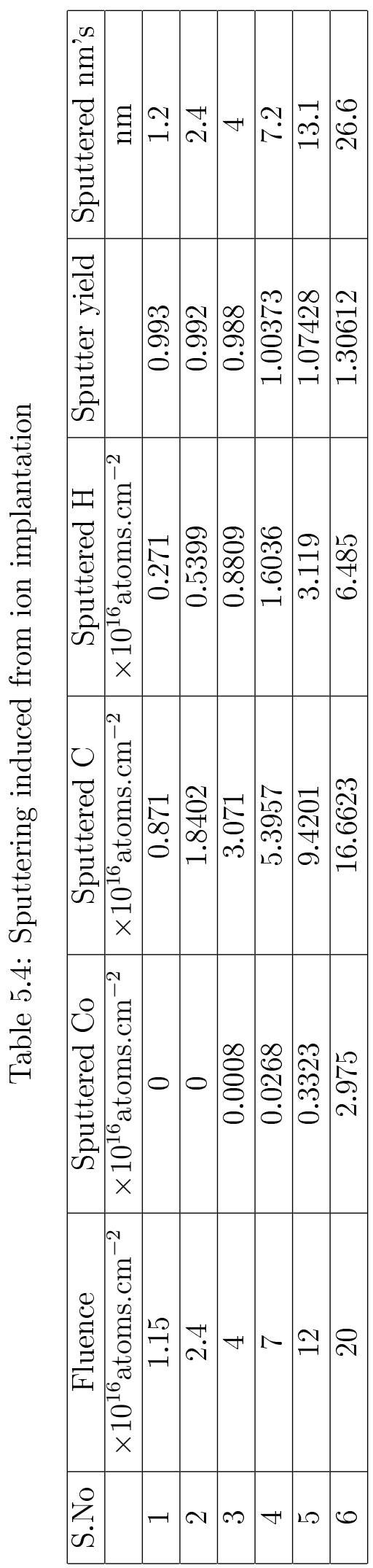


to reduce slightly. This can be attributed to the difference in sputter yield of $\mathrm{C}$ and $\mathrm{H}$. As carbon has higher sputter yield the surface gets preferentially depleted of carbon leading to hydrogen accumulation. The sputter yield of hydrogen is lower. This in turn lead to reduced sputtering at surface since a higher fraction of the surface is now covered by hydrogen.

However, it can be seen that the sputter yield increases beyond an implantation fluence of $4 \times 10^{16}$ atoms.cm ${ }^{-2}$. This is due to the presence of Co at the surface. The sputter yield of $\mathrm{Co}$ on $\mathrm{Co}$ is higher than both $\mathrm{C}$ and $\mathrm{H}$. This leads to increased sputtering leading to larger depletion. This trend is clearly visibly in table 5.4. The effect of sputtering is so high at $\mathrm{F}$ $=20 \times 10^{16}$ atoms. $\mathrm{cm}^{-2}$, that the sputtered layer is now $27 \mathrm{~nm}$ thick.

In summary, DTRIM predicts that the distribution of $\mathrm{Co}, \mathrm{C}$ and $\mathrm{H}$ in implanted DLC films. Co has maximum concentration around its mean projected range while $\mathrm{C}$ and $\mathrm{H}$ attain their minima at the same region. Co assumes a Gaussian distribution at lower fluence. As the fluence increases, the profile deviates slightly from the Gaussian distribution with higher accumulation of Co before $R_{p}$. Sputtering is observed at all fluences however they gain significance at higher implantation fluence. It was observed that increased sputtering shifts the implantation distribution towards the surface. This leads to higher concentration of implanted ions at the surface. It is important to remember that DTRIM bases its calculation entirely on the ballistics of the system and do not consider the effects of dynamic changes in density, precipitation or diffusion effects.

\subsection{Co distribution in implanted DLC}

The primary technique used to probe the distribution of implanted ions in DLC matrix was RBS. RBS has an advantage when the elements composing the material differ significantly in their mass, in particular involving high $\mathrm{Z}$ elements. In this case, the implanted ions, $\mathrm{Co}$, are much heavier than the elements composing the base matrix, $\mathrm{C}$ and $\mathrm{H}$. RBS cannot give much information about $\mathrm{C}$ and no direct information about $\mathrm{H}$ since they are light elements. However, it can measure the distribution of Co in DLC with great precision. In this study, high resolution RBS is used to probe the implanted ion distribution. 
Table 5.5: HR-RBS parameters

\begin{tabular}{|c|c|}
\hline Parameters & Values \\
\hline \hline Incident ion & ${ }^{4} \mathrm{He}^{+}$ \\
\hline Energy & $500 \mathrm{keV}$ \\
\hline Incident angle & $53.05^{\circ}$ \\
\hline Exit angle & $62.37^{\circ}$ \\
\hline Scattering angle & $65^{\circ}$ \\
\hline Calibration offset & $277.57 \mathrm{keV}$ \\
\hline Energy per channel & $0.4344 \mathrm{keV} / \mathrm{channel}$ \\
\hline Particles $*$ Sr & $2.626 \mathrm{E}+10$ \\
\hline Detector resolution & $1.700 \mathrm{keV}$ \\
\hline
\end{tabular}

Table 5.5 shows the typical settings used for the HR-RBS measurement. Conversion of the raw data to informative plots requires several steps which are detailed in section 3.4 of the thesis. In this section we jump directly to the processed data. The elements present in the samples are the implanted Co atoms, $\mathrm{C}$ and $\mathrm{H}$ from the DLC film and Si from the substrate. Except hydrogen all the other elements of interest can be detected in RBS. RBS can also detect presence of any oxygen contamination in the films. The scattering energy of elements of interest calculated based on their kinematic factor for a $500 \mathrm{keV} \mathrm{He}{ }^{+}$beam and $65^{\circ}$ scattering angle are presented in table 5.6.

Table 5.6: Kinematics for $500 \mathrm{keV} \mathrm{He}{ }^{+}$beam

\begin{tabular}{|c|c|}
\hline Element & Scattering Energy $(\mathrm{keV})$ \\
\hline \hline${ }^{12} \mathrm{C}$ & 336.6 \\
\hline${ }^{16} \mathrm{O}$ & 372.9 \\
\hline${ }^{28} \mathrm{Si}$ & 423.5 \\
\hline${ }^{59} \mathrm{Co}$ & 462.2 \\
\hline
\end{tabular}

The scattering energy of $500 \mathrm{keV} \mathrm{He}{ }^{+}$beam computed for each element in table 5.5 assumes the corresponding element to be present at the surface. If they are located deeper within the sample, then excess energy is lost in the path between the surface and that specific depth. Thus all elements present in the surface of the sample should give rise to a signal at their corresponding scattering energy presented in table 5.6.

The data gathered from the HR-RBS measurement usually gives rise to a spectrum as shown in figure 5.4. 


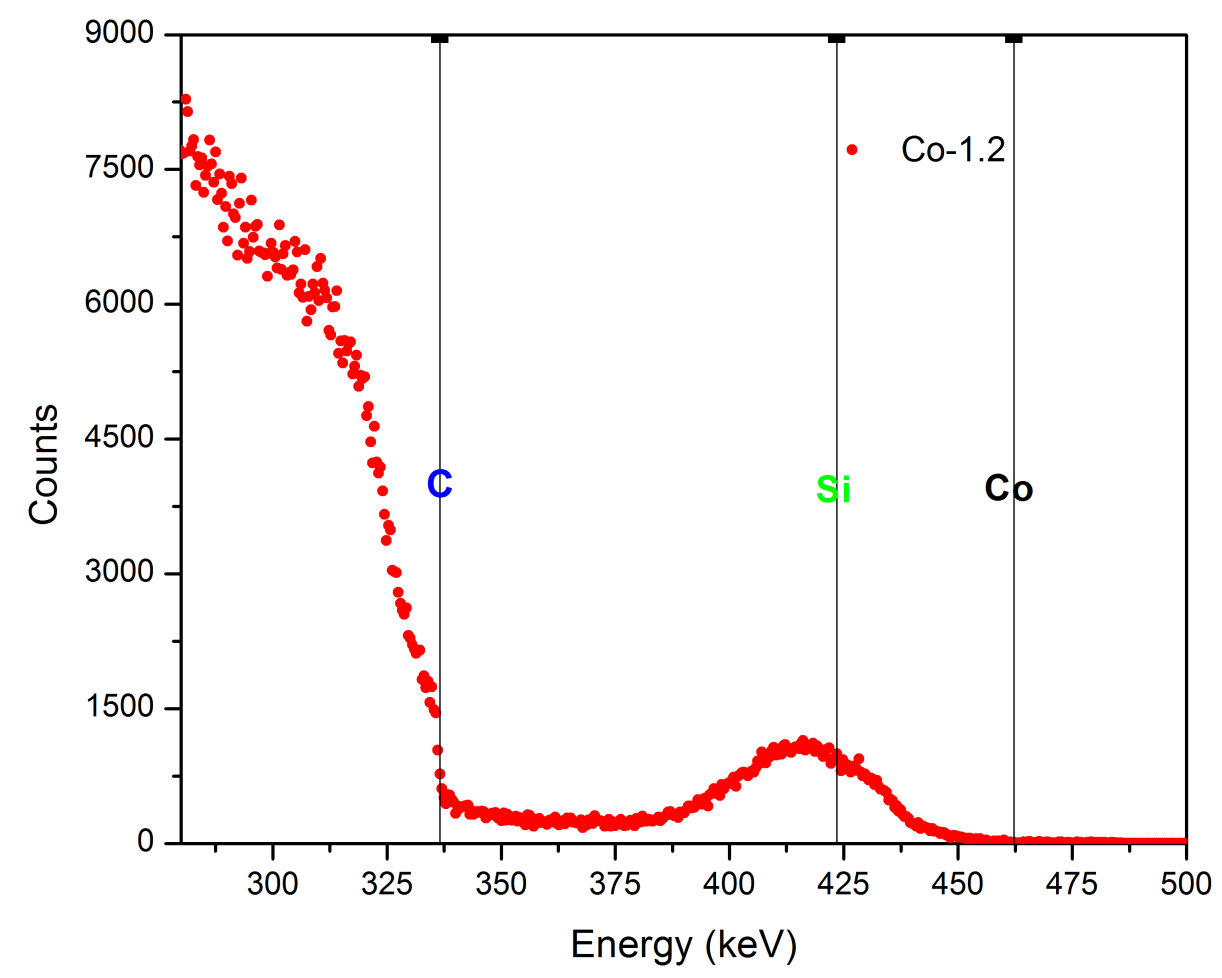

Figure 5.3: HR-RBS spectrum of Co 1.2. The position at which signals from $\mathrm{C}, \mathrm{Si}$ and $\mathrm{Co}$ should arise if located at surface is marked in the figure. It can be observed that $\mathrm{C}$ is present at the surface and Co is present close to the surface.

In the figure, the information is presented in a counts vs energy plot. The abscissa represents the scattering energy range of the $\mathrm{He}^{+}$particles. This scattering energy corresponds directly to the mass and position of the detected element. The ordinate, corresponds to the number of $\mathrm{He}^{+}$particles detected at that particular scattering energy. This directly corresponds to the concentration of the element at that particular depth. The scattered $\mathrm{He}^{+}$ particles are referred here onward as signals in the HR-RBS spectrum.

The HR-RBS spectrum in figure 5.3 represents the distribution from Co 1.2 sample. Signals are recorded starting from an energy of $486 \mathrm{keV}$. However, the signal strength is within the noise level (counts: $\sim 10$ ) and hence do not correspond to any particular element. These signals are expected to rise from multiple scattering effects or can just be noise from the detector. A rise in the signal strength is observed starting from $\sim 462 \mathrm{keV}$. The heaviest element expected in the sample is Co. Its surface scattering energy as computed in table 5.6 corresponds to $462.2 \mathrm{keV}$. Thus this signal can be attributed to originate from Co. This signal is observed to assume a nearly Gaussian distribution extending up to $\sim 380 \mathrm{keV}$. The 
signal however does not drop to zero. It stabilizes around 200 counts until the arrival of the next major feature starting at $\sim 336 \mathrm{keV}$. The signal originating at this energy can be attributed to carbon, based on the kinematics denoted in table 5.6. This is expected since carbon is present at the surface of the sample. This feature is immediately followed by a large rise in signal at around $325 \mathrm{keV}$. This signal continue to rise with decrease in scattering energy. The only element in the sample that can be detected with such large intensities is Si. Silicon's surface scattering energy is $423.5 \mathrm{keV}$. However the substrate is preceded by the DLC film containing Co. Thus the Si signal corresponds to the interface between the DLC and the Si substrate. The slope of the Si signal denotes the intermixing between the $\mathrm{Si}$ and C layer. Unfortunately, the overlay of Si signal on C signal prevents us from further interpreting the $\mathrm{C}$ signal. The main information that can be gathered from this measurement is the distribution of implanted ions in the DLC matrix. Plenty of its features still remain unexplained. A much vigorous quantitative treatment of the HR-RBS spectrum is required to extract the depth profile of the implanted ion and explain the different features of the spectrum.

For this purpose, a simulation software is used to assess the experimental data obtained from RBS. The purpose of the software is to simulate an RBS spectrum equivalent to the experimental data based on the input parameters. These input parameters consist of both the operational settings associated with the instrument and the composition information about the target sample. For example, one can construct a target with an assumed concentration and depth profile. Then the software can simulate an RBS spectrum of the constructed target based on the kinematics involved in the measurement. This step requires information about the operational conditions applied in the experiment.

The simulated spectrum can then be compared with the experimental data. The input parameters can be tuned in an iterative manner to obtain a simulated spectrum that aligns best with the experimental data. Since the experimental parameters such as ion energy, detection angle etc., are known variables they are assigned a constant value in the simulation. The target composition and depth profile are the unknown data which are continuously refined in the simulation input to obtain the best match between the simulation and the experiment. The target composition and depth profile that corresponds to the best match can be taken to represent the measured sample. In this chapter, SIMNRA software is used for extracting information from the RBS spectra.

Consider the RBS spectrum from sample Co 1.2. The operational parameters of the measurement and the raw data are loaded into the SIMNRA software as shown below. 


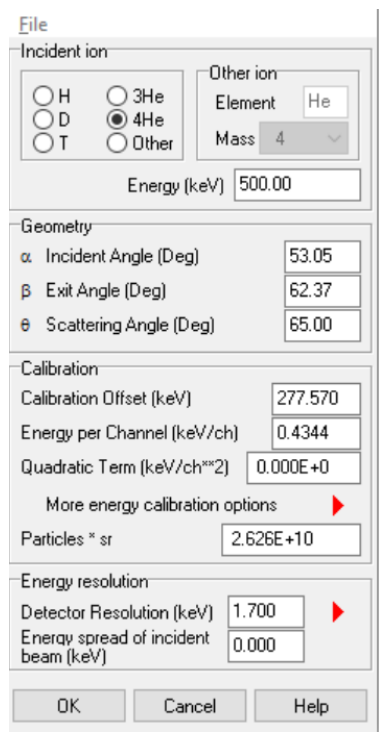

(a) Experimental setup

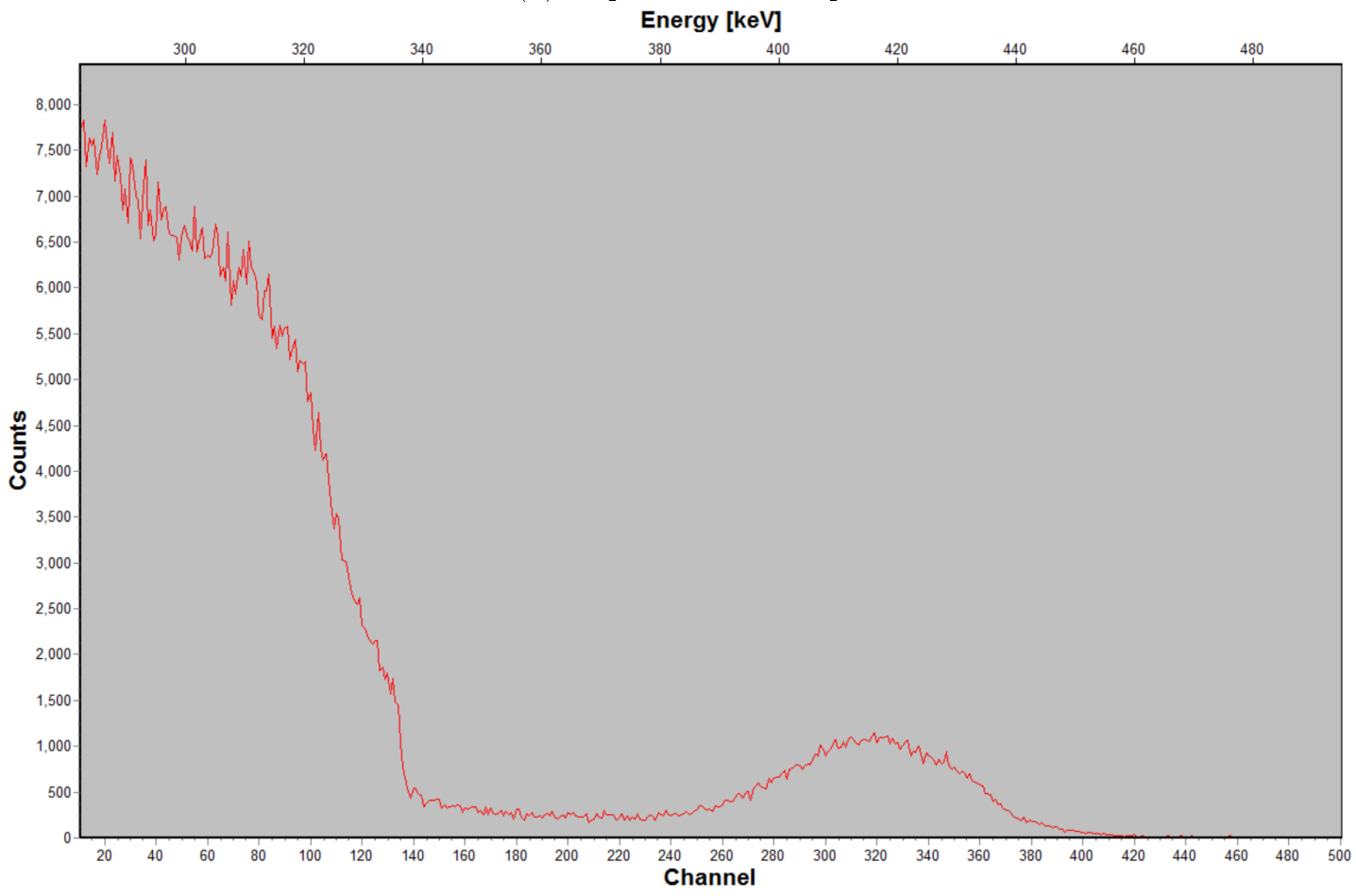

(b) Raw data

Figure 5.4: Screenshots of the SIMNRA program used for data analysis. The experimental setup and raw data of Co 1.2 sample are displayed.

The next step is to construct a target whose simulated profile will match the experimental data. This requires a number of iterative steps in a trial and error fashion. DTRIM simulations are very useful in this step since they predict the expected distribution and can thus help in constructing the target. This process becomes more complicated for high resolution RBS since the information captured is very sensitive to changes in composition for every 
nanometer. This leads to a difficulty in simulating the curvature of the peaks observed in the spectrum. This can be illustrated in the fits below. 


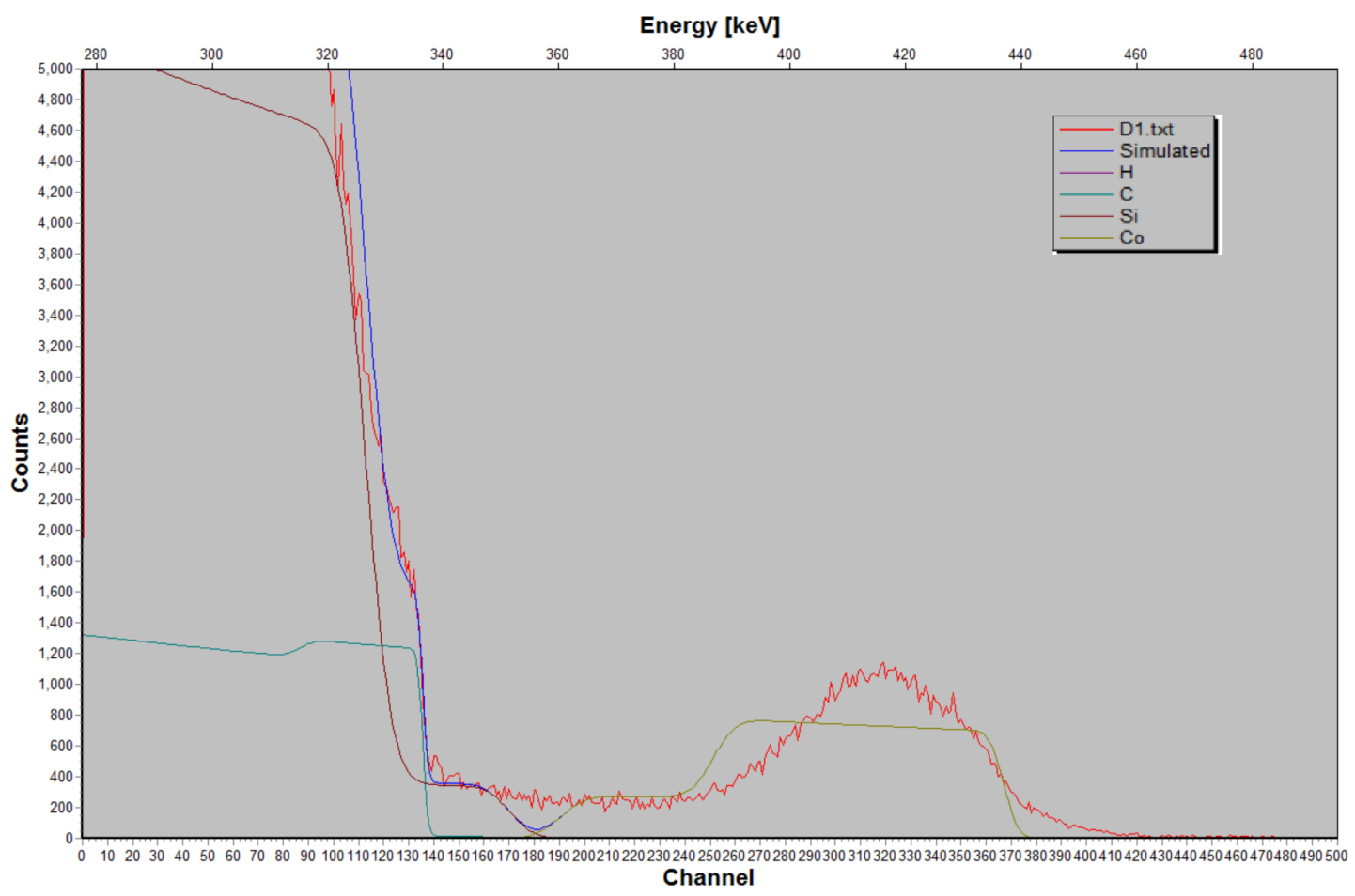

(a) Initial fit

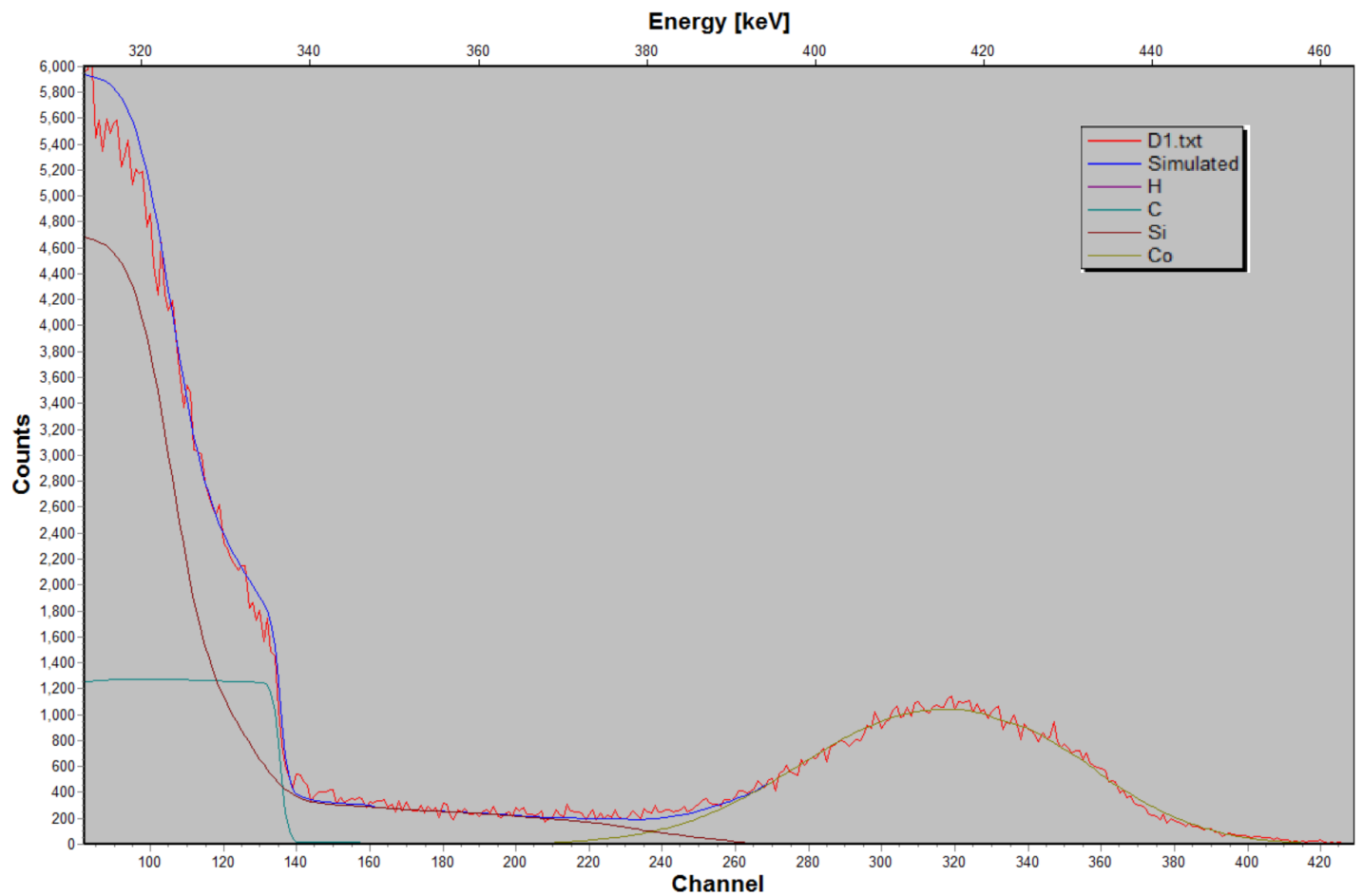

(b) Best fit

Figure 5.5: SIMNRA fitting of HR-RBS spectrum of Co 1.2 sample. The figure illustrates the necessity for detailed target construction for HR-RBS. Figure 5.5 (a) shows the data from Co 1.2 sample fit with only 4 layers while figure 5.5 (b) shows the same data fit with 27 layers. It can be seen that detailed target construction can fit the HR-RBS data accurately and it allows extraction of precise details measured by the high-resolution setup. 
Table 5.7: SIMNRA target summaries of Co 1.2 HR-RBS spectrum

(a) Elemental composition of SIMNRA target

\begin{tabular}{|c|c|c|}
\hline Element & Initial fit $\left(\times 10^{15}\right.$ atoms.cm $\left.^{-2}\right)$ & Best fit $\left(\times 10^{15}\right.$ atoms.cm \\
\hline
\end{tabular}

(b) No of layers for each fit

\begin{tabular}{|c|c|}
\hline Fit & No. of layers \\
\hline \hline Initial & 4 \\
\hline Best & 27 \\
\hline
\end{tabular}

Figure 5.5, shows the same experimental data fit with two different simulations. Both the simulations have the same target composition. This can be seen from table 5.7. However, the fit qualities are vastly different. This is attributed to the level of detail put into the target description. The depth resolution of HR-RBS measurement is in the order of $\sim 1-2 \mathrm{~nm}$ which worsens at greater depth due to energy straggling. The distribution of Co in the above spectrum assumes a Gaussian shape covering about 40 - $60 \mathrm{~nm}$ as per DTRIM simulations. Since the Co concentration exhibit continuous change over the depth, it would require a multiple number of target layers to simulate the profile The required number of layers to simulate the above distribution can approximately be given by dividing the distribution width by the depth resolution, which in this case ranges from $20-30$.

Figure 5.5 (a) shows a SIMNRA fit constructed with 4 target layers and 5.5 (b) shows the same data fit with a target composed of 27 layers. While the latter process can be more time consuming, the advantage of using this procedure is it extracts details about the very fine features of the elemental distribution. These features which are usually not identified in normal RBS measurements can be precisely quantified by this technique. The significance of this advantage in our work will be detailed in the below paragraphs. Once the simulations match the experimental data set, Co distribution in the target constructed in SIMNRA can be taken for all practical purposes, to represent the Co distribution in sample Co 1.2. In similar fashion all the samples measured with HR-RBS are fit with appropriate simulations and the corresponding Co profiles are extracted for our analysis. 


\subsubsection{Low fluence - Unimodal distribution}

Co distribution extracted from the above fit is presented in figure 5.6.

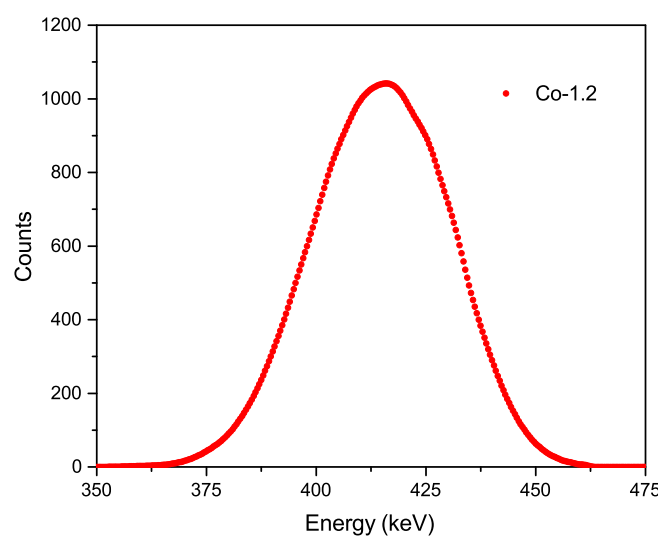

(a) Co profile from HR-RBS spectrum

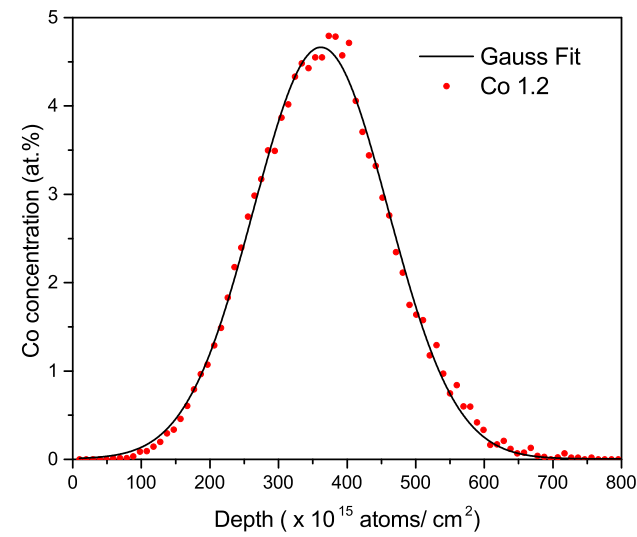

(c) DTRIM prediction of Co depth profile

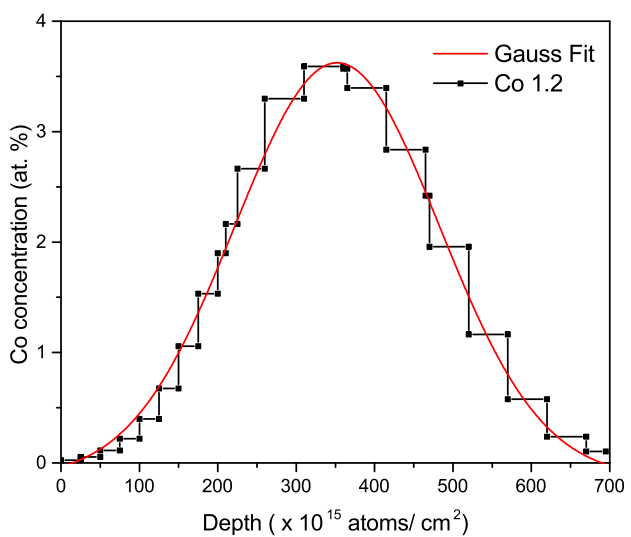

(b) SIMNRA depth profile of Co distribution

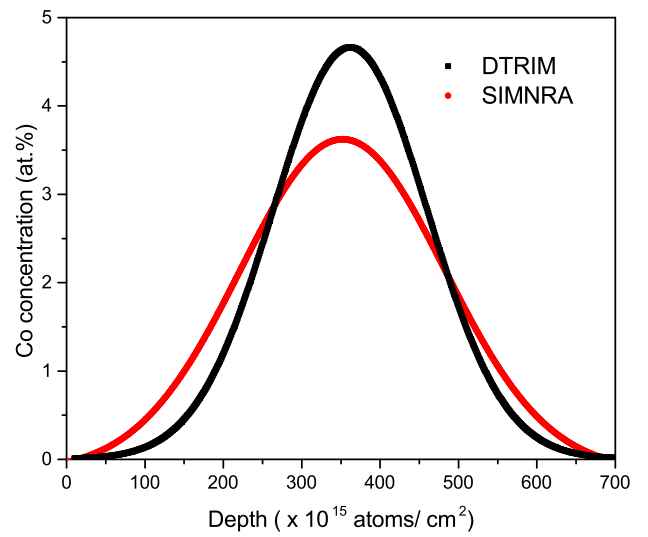

(d) DTRIM Simulation vs Experimental measurement

Figure 5.6: Analysis of HR-RBS spectrum of Co 1.2. The data analysis reveals that the Co distribution for Co 1.2 assumes a Gaussian distribution with a peak concentration of 3.75 at.\% and it does not completely align with the theoretical prediction.

Figure 5.6 (a) shows the profile of Co distribution with respect to energy. The plot was directly extracted from the SIMNRA fit (Co layers) of Co 1.2 shown earlier. The Co distribution is described in a counts vs energy plot. As mentioned earlier, the counts correspond directly to the concentration of Co present in a layer and the energy corresponds directly to the depth scale. The distribution of Co is observed to start from $462 \mathrm{keV}$ which corresponds to Co surface scattering energy under the given conditions. However, no significant surface Co concentration was detected. The distribution is Gaussian in nature with a mean projected range corresponding to a scattering energy of $416 \mathrm{keV}$. 
Around $425 \mathrm{keV}$, there is minor change in the curvature of the distribution. Since the observed shift in curvature is within the noise of the instrument, it cannot be attributed to any real change in Co distribution. It is worth mentioning at this point that figure 5.6 (a) is the most truthful representation of Co distribution in Co 1.2. This is because conversion of the energy scale to depth scale would involve assumptions about the composition and density of the target sample. However, the plot in its current format prevents any useful quantitative interpretation that is needed for this research. Hence we apply the conversion of energy scale to depth scale.

Figure 5.6 (b) shows the same profile of Co in Co 1.2 in the form of a concentration vs depth plot where the concentration is represented in units of atomic percentage and depth in $10^{15}$ atoms. $\mathrm{cm}^{-2}$. The profile is extracted from the SIMNRA analysis shown earlier. Depth in $10^{15}$ atoms.cm ${ }^{-2}$ finds standard usage in ion beam processes and analyses. Since there is no information yet about the density of the material it is not possible to show the same depth in nanometers. If the atomic density of the target material is known then depth in nm can be obtained as follows,

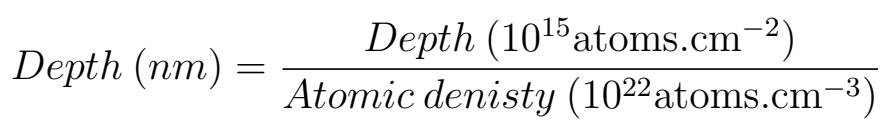

If for example, the atomic density of the implanted DLC is $1 \times 10^{23}$ atoms.cm $^{-3}$, then 1 $\mathrm{nm}$ would correspond to $1 \times 10^{16}$ atoms.cm ${ }^{-2}$.

Every layer of the target is represented by two data points in figure 5.7 (b). The first data point denotes the beginning of the layer and the second corresponds to the end of layer. Within this layer Co is assumed to have a constant concentration. For example, consider the first layer. It starts from the surface, i.e., value 0 in depth scale and extends up to $25 \times 10^{15}$ atoms.cm ${ }^{-2}$. This layer is constructed to have a Co concentration of 0.025 at.\%. In figure 5.6 (b) this layer is represented by the first two data points with $\mathrm{X}$ value 0 and $25 \times 10^{15}$ atoms.cm ${ }^{-2}$ and a constant Y value of 0.025 at.\%. Similarly all the layers of the target are represented by two such data points. The change in Co concentration from one layer to the other is represented by sudden steps of increase or decrease in Co concentration. While such a construction can in principle give rise to the HR-RBS spectra as shown in figure 5.6 (a), it does not represent reality. Co is expected to continuously change with depth. While the step function may represent the overall Co distribution and concentration of the sample the changes in Co concentration within a layer and in between layers can best be represented by a continuous function. The plot is thus fit with a Gaussian distribution to approximate the changes in Co concentration with depth. This fit now describes the change in Co concentration as a function of depth (in $10^{15}$ atoms.cm ${ }^{-2}$ ). 
In an earlier section, DTRIM simulations were employed to predict the distribution of implanted Co in DLC. The predicted profile can now be compared with the experimental profile to identify any changes between them. Any significant change if present, would force us to re-evaluate the approximations placed in DTRIM simulation. In figure 5.2 (a), Co depth distribution predicted for Co 1.2 is shown. The plot describes the change in Co concentration as a function of depth in $\mathrm{nm}$. As already mentioned, DTRIM calculations are purely based on the ballistics of ion implantation and does not take into account any changes in the atomic density during implantation. Hence, the final distribution is obtained considering the original atomic density. The input file of DTRIM contains the atomic density of DLC which is $9.82 \times 10^{22}$ atoms.cm ${ }^{-3}$. By considering this density value, depth in $10^{15}$ atoms.cm $^{-2}$ can be calculated from depth in nm. Figure 5.6 (c) shows the predicted Co distribution with depth scale in $10^{15}$ atoms.cm ${ }^{-2}$. The predicted Co depth distribution is further fit with a Gaussian distribution to obtain a function describing the continuous changes in Co concentration with depth.

Finally, the experimental SIMNRA fit can now be compared with the theoretical DTRIM fit as shown in figure 5.6 (d). The figure shows that the predicted distribution does not align completely with the measured distribution. The peak concentration of the measured distribution is found to be lower than that predicted by DTRIM. A minor concentration of Co is observed in near-surface region outside the predicted range. To quantify these changes the fitting function and its associated parameters have to be considered. The equation describing the fitting function is shown below,

$$
y=y_{0}+\frac{A}{w \cdot \sqrt{(\Pi / 2)}} \cdot \exp \left(\frac{-2\left(x-x_{c}\right)^{2}}{w^{2}}\right)
$$

where $y=$ Co concentration, $y_{0}=$ Constant, $A=$ Area under the curve which here refers to the implantation dose, $w=$ width of the distribution and in this case $2 *$ straggle, $x=$ depth and $x_{c}=$ mean projected range.

Table 5.8 shows the parameters of the Gauss fit describing the experimental data and theoretical predictions. 
Table 5.8: Fitting parameters of Co 1.2: Experiment vs Theory

\begin{tabular}{|c|c|c|c|c|c|}
\hline Parameters & Units & Experiment & Fitting error & Theory & Fitting error \\
\hline$y_{0}$ & at.\% & c -0.131 & c0.10 & 0.005 & 0.014 \\
\hline$A$ & $\times 10^{15}$ atoms.cm $^{-2}$ & 12.27 & 0.73 & 11.45 & 0.10 \\
\hline$w$ & $\times 10^{15}$ atoms.cm $^{-2}$ & 260 & 11 & 196 & 2 \\
\hline$x_{c}$ & $\times 10^{15}$ atoms.cm $^{-2}$ & 352 & 3 & 362 & 1 \\
\hline Straggle & $\times 10^{15}$ atoms.cm $^{-2}$ & 130 & 6 & 98 & 1 \\
\hline FWHM & $\times 10^{15}$ atoms.cm $^{-2}$ & 307 & 13 & 230 & 2 \\
\hline Peak Concentration & at.\% & 3.75 & 0.11 & 4.66 & 0.03 \\
\hline
\end{tabular}

Direct comparison of the parameters of experimental and theoretical fits clearly illustrates the quantitative difference between them. The implantation dose from the experimental fit is comparable to the theoretical distribution within the error margin. Hence it is logical to compare both the distributions. Two differences can be identified from this comparison. First is the straggling or the standard deviation of the distribution. The measured Co distribution is more disperse than the predicted distribution. This in principle contributes towards the lowering of the peak concentration. The other key difference lies in the mean projected range of the measured and predicted Co distribution. The mean projected range measured is closer to the surface than that predicted by DTRIM.

Before attempting to explain the observed differences, it is necessary to put the observed differences in a more familiar perspective. For this purpose, an assumption is made on the atomic density of the implanted films. The implanted film is assumed to have the same atomic density as of the as-deposited film. Even though it is not accurate the assumption is made to enable comparison since DTRIM simulations makes the same assumption. The asdeposited film was measured to have a density of $9.82 \times 10^{22}$ atoms.cm ${ }^{-3}$. This density value results in the following equivalence,

$$
0.98 \mathrm{~nm}=1 \times 10^{16} \text { atoms.cm }^{-2}
$$

Thus the measured straggling is greater than the predicted straggling by $\sim 3 \mathrm{~nm}$ and the mean projected range is measured to be lesser than the predicted distribution by $\sim 1$ nm. The differences are not huge. Usually, such differences are considered to be within the experimental errors. The difference in mean projected range especially is within the detector's resolution and hence need not be further investigated. However, the issue of straggling is different.The difference in straggling is well above the detector's resolution since the measurement is performed by HR-RBS with $1.1 \mathrm{~nm}$ resolution. While the difference may look small, as per DTRIM simulation this difference is quantitatively similar to the difference in straggling observed between an implantation fluence of 1.2 and $20 \times 10^{16}$ atoms.cm ${ }^{-2}$ (shown in table 5.3). 
In retrospect to the results obtained from the other samples, it becomes redundant to analyze these differences in detail at this point. In fact, in the next immediate subsection the nature of mismatch between the measured and the predicted distribution becomes abundantly clear. A detailed discussion on the possible causes for this mismatch is provided at the end of this section.

\subsubsection{Moderate fluence I : Emergence of bimodality}

In the previous section, taking Co 1.2 as an example, the different steps required to process the data to obtain useful information about the implanted ion distribution was presented. The measured Co distribution for Co 1.2 was also compared with the predicted DTRIM distribution obtained in section 5.2. Some differences between the measured and predicted data were identified. In order to better understand the nature of these differences, results from DLC implanted with higher fluence are to be considered.

Before analyzing these results it would be useful to make few predictions. The source of mismatch between the measured and theoretically predicted Co distribution could arise either from, experimental errors or from the limitations of the DTRIM simulations. If the difference is caused by an experimental error or uncertainty then it is less likely for it be present in a sample implanted and measured at a different time. On the other hand, if the deviation from DTRIM predictions can be associated with the limitation of the DTRIM simulations, then it is more likely for this effect to amplify with increase in fluence. In this subsection the results from the HR-RBS measurement of the Co 2.4 sample are presented.

A similar procedure was carried out to fit the Co 2.4 data. The fitting of SIMNRA target with the experimental data set along with the details of the constructed data are shown in figure 5.7 and table 5.9. 


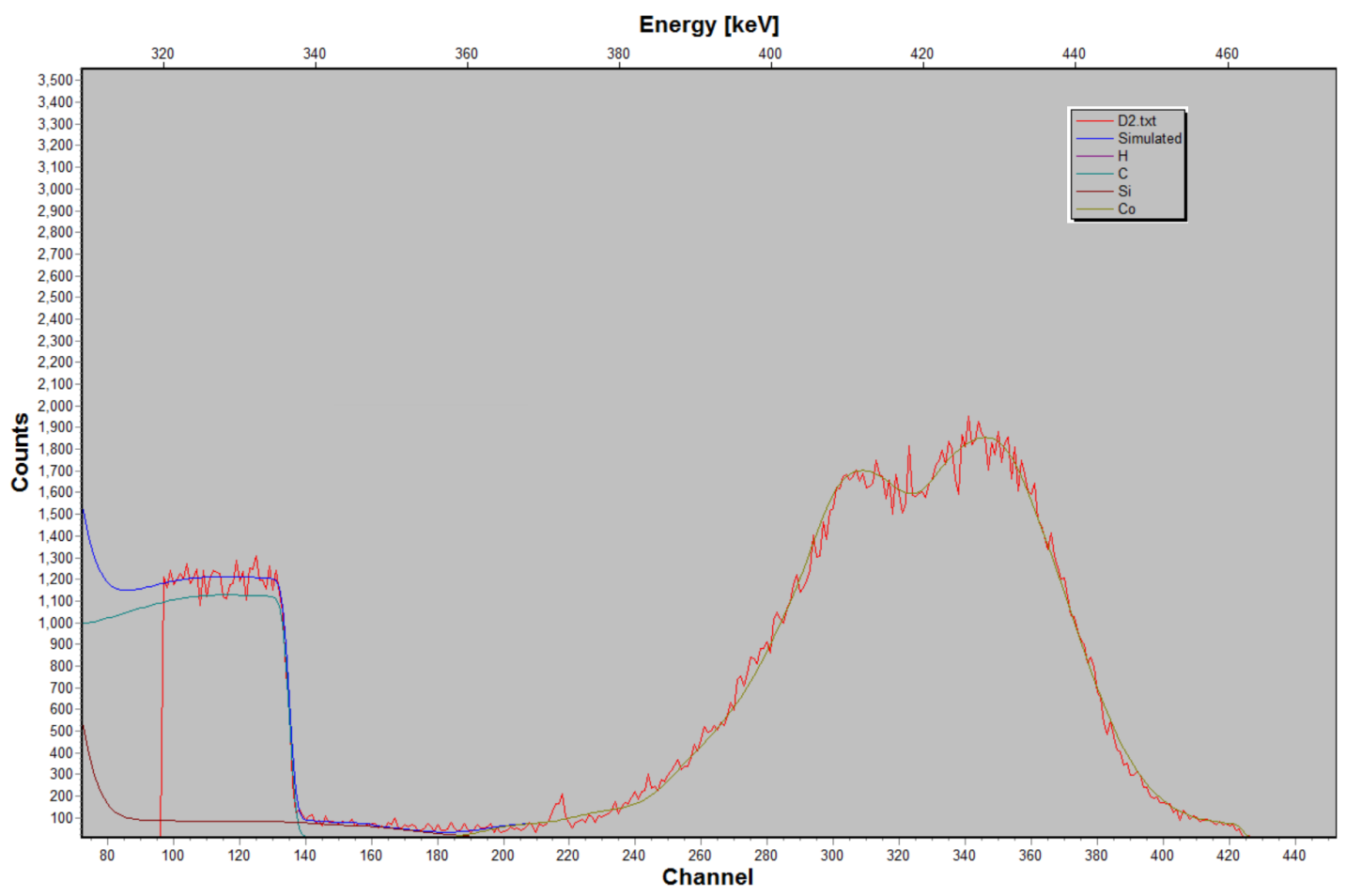

Figure 5.7: SIMNRA fitting of Co 2.4 HR-RBS spectrum measuring the bimodal Co distribution in DLC

Table 5.9: Co 2.4 SIMNRA Target composition

\begin{tabular}{|c|c|}
\hline Element & Concentration $\left(\times 10^{15}\right.$ atoms.cm $\left.^{-2}\right)$ \\
\hline \hline $\mathrm{C}$ & 728.8 \\
\hline $\mathrm{H}$ & 218.2 \\
\hline $\mathrm{Co}$ & 24.0 \\
\hline $\mathrm{Si}$ & 100000 \\
\hline
\end{tabular}

Even before constructing the SIMNRA target, just from observing the HR-RBS spectra of Co 2.4 the uniqueness of the measured Co distribution is abundantly clear. Instead of a unimodal distribution, the implanted Co exhibit a bimodal distribution. This is an unexpected and a significant finding since Co implantation was carried out at room temperature and at a single implantation energy. To understand the implications of the above result, it is useful to briefly revisit the theory of ion implantation. Ion implantation is a process in which energetic ions are bombarded onto a target surface. Upon entering the sample target, the ion loses energy by two mechanisms: electronic and nuclear energy losses. The inelastic scattering of the energetic ions with the electrons of base matrix contributes towards electronic stopping. The other mechanism is loss of energy through collisions between the energetic ions and the atoms composing the base matrix. This is referred to as nuclear 
stopping. After a number of collisions the ion loses enough energy rendering it unable to move further within the matrix. The final position it comes to rest withing the base matrix depends on the number of collisions it experiences within the material. Since this process involves a large number of ions each undergoing collisions with a large number of atoms, it qualifies as a statistical process with the range of the implanted ions following nearly a Gaussian distribution with a single mean projected range.

In other words, the average distance traveled by the ion inside the matrix depends on the average number of collisions it experiences. Thus there must exist a mean projected range where maximum number of implanted ions comes to rest. Any position away from the mean projected range should have lower number of Co atoms with the distribution being a function of position with respect to the mean projected range. Figure 5.7 seems to suggest that the Co distribution has two mean projected range. There is no theoretical basis or experimental evidence for mono-energetic implantation to lead to such a distribution with multiple mean projected range. However, at the same time it is a fact that we observe the distribution of implanted Co in DLC with two maxima. At this stage there is not enough information to draw any conclusions. More information and analysis is required to provide a clear explanation. At this point, the purpose of these subsections is to clearly identify and if possible quantify these features. 


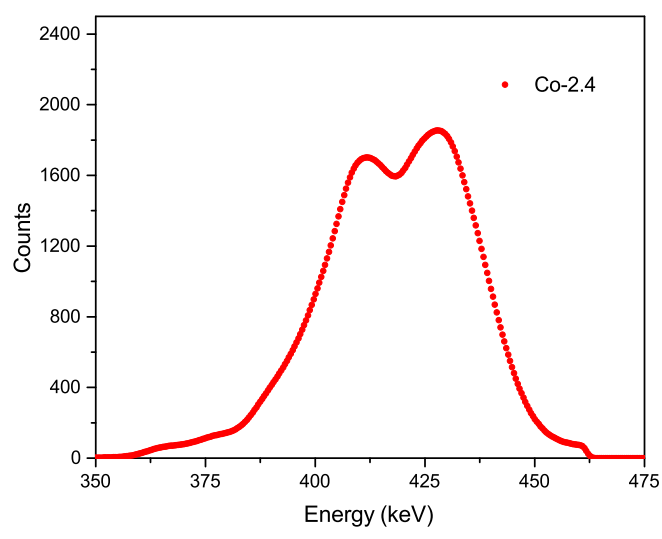

(a) Co profile from HR-RBS spectrum

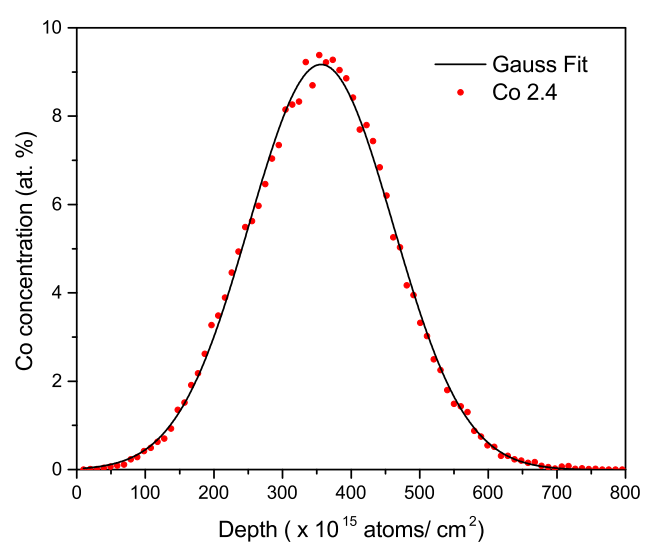

(c) DTRIM prediction of Co depth profile

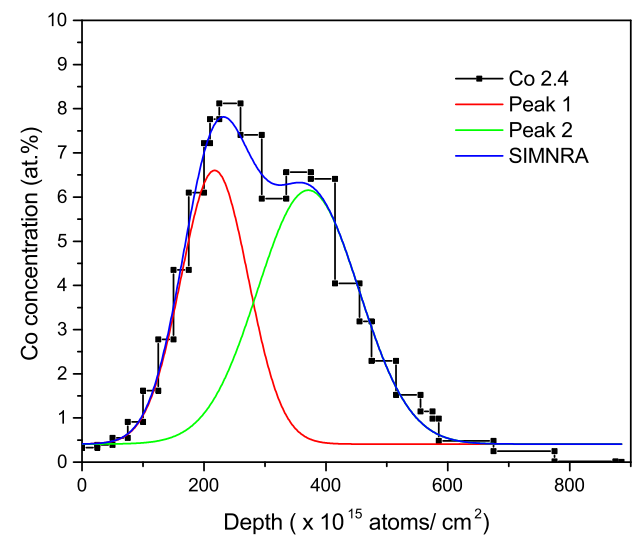

(b) SIMNRA depth profile of Co distribution

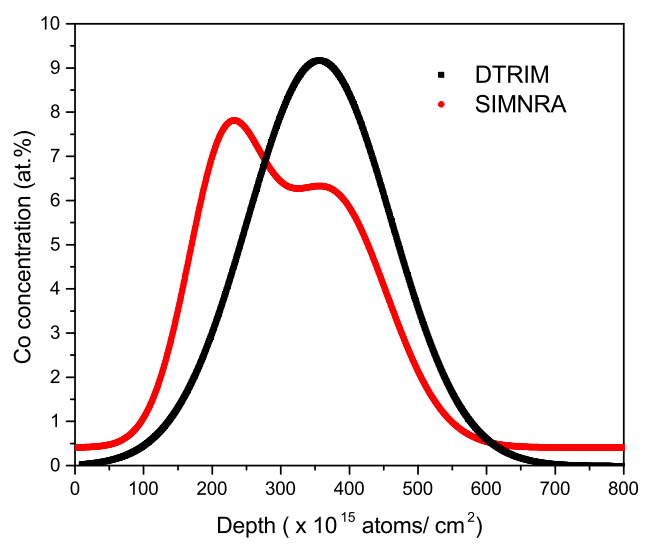

(d) DTRIM Simulation vs Experimental measurement

Figure 5.8: Analysis of HR-RBS spectrum of Co 2.4. The data analysis reveals that the Co distribution for Co 2.4 assumes a bimodal distribution in contrast to the theoretically predicted unimodal distribution.

The analysis of the Co distribution in Co 2.4 begins from extraction of Co data from the SIMNRA simulation. Figure 5.8 (a) shows the Co distribution in a counts vs energy plot which allows encapturing the fine details from the measurement. This plot clearly shows the gradual decrease in Co concentration from the first peak at $428 \mathrm{keV}$ till it reaches a minima at $418 \mathrm{keV}$. This trend is followed by a similar gradual increase in Co concentration which finally maximizes at $412 \mathrm{keV}$. Furthermore, the Co concentration is measured to have a long tail near the end of distribution. The nature of this elongated distribution is best represented in this plot.

Figure 5.8 (b) shows the variation of Co concentration in implanted DLC as a function of depth in $10^{15}$ atoms.cm ${ }^{-2}$. The maximum Co concentration was observed to be 8.12 at. $\%$ in between 225 -260 $\times 10^{15}$ atoms.cm ${ }^{-2}$ The second peak was observed in the region ranging 
from $335-375 \times 10^{15}$ atoms.cm ${ }^{-2}$ with a Co concentration of 6.57 at.\%. The minima in between these peaks is located at $295-335 \times 10^{15}$ atoms.cm ${ }^{-2}$ with a Co concentration of 5.96 at.\%. Thus the first inference from this plot is that this observed variation in Co distribution is above the limit of detection of HR-RBS confirming the bimodal nature of Co distribution. The second noticeable feature is the presence of Co $(0.4$ at.\%) at the surface layer. This can actually explain the small peak at $373 \mathrm{keV}$ observed in figure 5.7. The peak corresponds to the surface scattering energy of oxygen. This peak was not observed in HR-RBS spectra of as-deposited DLC or in Co 1.2 sample. This indicates that Co present at the surface oxidizes and leads to formation of an oxide layer. The measured distribution is then fit with multiple Gaussian distribution which cumulatively fits the step-wise distribution. The fitting provides a mathematical description of the measured Co distribution as a function of depth. The fit parameters are shown in table 5.10.

Table 5.10: Co 2.4 Experiment vs Theory

\begin{tabular}{|c|c|c|c|c|}
\hline Parameters & Units & Experiment & Experiment & Theory \\
\hline & & Peak 1 & Peak 2 & \\
\hline$y_{0}$ & at. $\%$ & 0.41 & 0.41 & 0.01 \\
\hline$A$ & $\times 10^{15}$ atoms.cm $^{-2}$ & 858 & 1198 & 2411 \\
\hline$w$ & $\times 10^{15}$ atoms.cm ${ }^{-2}$ & 111 & 166 & 210 \\
\hline$x_{c}$ & $\times 10^{15}$ atoms.cm ${ }^{-2}$ & 218 & 371 & 356 \\
\hline Straggle & $\times 10^{15}$ atoms.cm $^{-2}$ & 55 & 83 & 105 \\
\hline FWHM & $\times 10^{15}$ atoms.cm $^{-2}$ & 130 & 196 & 247 \\
\hline Peak Concentration & at. $\%$ & 6.19 & 5.74 & 9.18 \\
\hline
\end{tabular}

Figure 5.8 (c) shows the Co distribution predicted from DTRIM for a fluence of 2.4 $\times 10^{16}$ atoms. $\mathrm{cm}^{-2}$. The distribution can be fit with a single Gaussian distribution very well. The distribution is unimodal with a mean projected range of $356 \times 10^{15}$ atoms.cm $^{-2}$ and a peak concentration of 9.17 at.\%. The straggling of the distribution was computed to be $105 \times 10^{15}$ atoms.cm ${ }^{-2}$. This fit can now be compared with the fit of the bimodal distribution. This comparison is shown in figure 5.8 (d). The differences between the theoretical computation and the experimental data can now be clearly seen. The differences are,

1. Experimental data shows a bimodal distribution while DTRIM predicts a unimodal distribution.

2. DTRIM does not predict Co to be at the surface while HR-RBS measures Co at the surface.

3. About $20 \%\left(0.48 \times 10^{16}\right.$ atoms.cm $\left.{ }^{-2}\right)$ of the implanted dose lies outside the predicted region. 
4. The maxima of the measured distribution is located about $120 \times 10^{15}$ atoms.cm $^{-2}$ before the predicted mean projected range. However, it is interesting to note that the second peak of the measured distribution coincides with the peak of predicted distribution.

5. The long tail of measured Co distribution is not accounted by DTRIM.

It is now worth the effort to look back at the analysis of Co 1.2 sample. It was found that the measured distribution was broader than the predicted distribution especially towards the surface. Earlier it was not possible to discuss the cause of these differences since it can also potentially be a measurement artifact. But results from Co 2.4 sample reaffirms the validity of the measurement. The observed differences in both the samples can either originate from use of a wrong parameter in the experiment or could be attributed to a cause not computed by the DTRIM simulation.

If the bimodal distribution can be caused by an experimental error, it should be possible to guess the nature of the error. The data from the two samples suggests that as the implantation fluence is increased from 1.2 to $2.4 \times 10^{16}$ atoms.cm ${ }^{-2}$ a bimodal distribution emerges from a unimodal distribution. In fact, the onset of bimodality is already observed as the broadening of the Co distribution measured in Co 1.2. For the ease of discussion, the peak observed closer the surface will here onward be referred to as the "surface peak" and the peak further from the surface located near the mean projected range as the "center peak".

At this stage, only two of the four prime Co implanted samples are presented. Results from the other samples sheds more light on these observations and dispel any doubts concerning the reality of these phenomenon.

\subsubsection{Moderate fluence II: Asymmetric bimodal distribution}

The next logical sample to investigate is DLC films implanted with Co to a fluence of $4 \times 10^{16}$ atoms.cm ${ }^{-2}$. If deviations from predicted distribution is expected to increase with implantation fluence, then more information about the bimodal distribution and its nature can be obtained from this sample. Same procedure is applied to the measured data to extract the required information. First, is the SIMNRA fitting of the sample HR-RBS data which is shown in figure 5.9 and table 5.11 . 


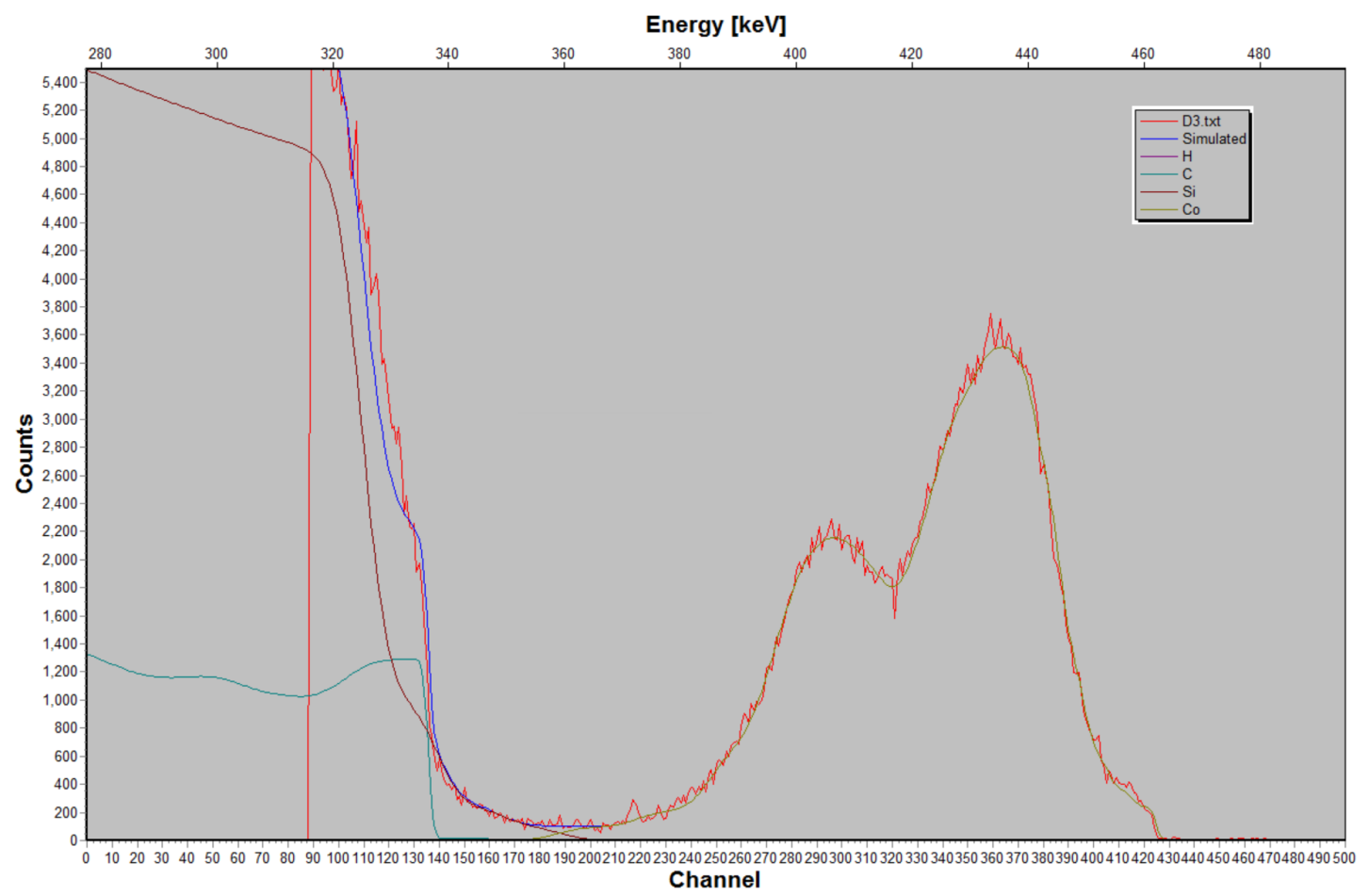

Figure 5.9: SIMNRA fitting of Co 4 HR-RBS spectrum measuring the asymmetric bimodal Co distribution in DLC.

Table 5.11: Co 4 SIMNRA Target composition

\begin{tabular}{|c|c|}
\hline Element & First attempt $\left(\times 10^{15}\right.$ atoms.cm $\left.^{-2}\right)$ \\
\hline \hline $\mathrm{C}$ & 548.2 \\
\hline $\mathrm{H}$ & 175.0 \\
\hline $\mathrm{Co}$ & 38.30 \\
\hline $\mathrm{Si}$ & 100000 \\
\hline
\end{tabular}

Figure 5.9 shows the HR-RBS spectrum of Co 4 with SIMNRA fit. The immediate observation is the prominent bimodal distribution with a strong surface peak. The raw data by itself strengthens our previous inference that the distribution is indeed bimodal. This SIMNRA fitting requires 24 layers with an overall Co dose of $3.8 \times 10^{16}$ atoms.cm $^{-2}$. The details are given in table 5.11. Once a satisfactory fit is obtained, the analysis procedure used previously is applied to extract useful information from the spectrum. 


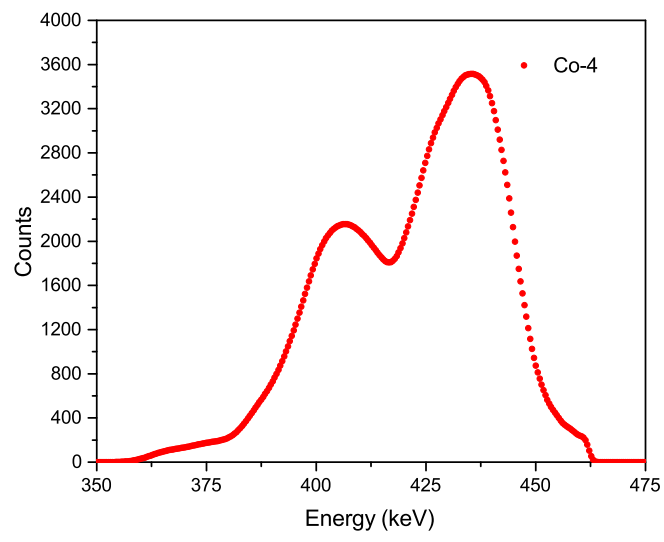

(a) Co profile from HR-RBS spectrum

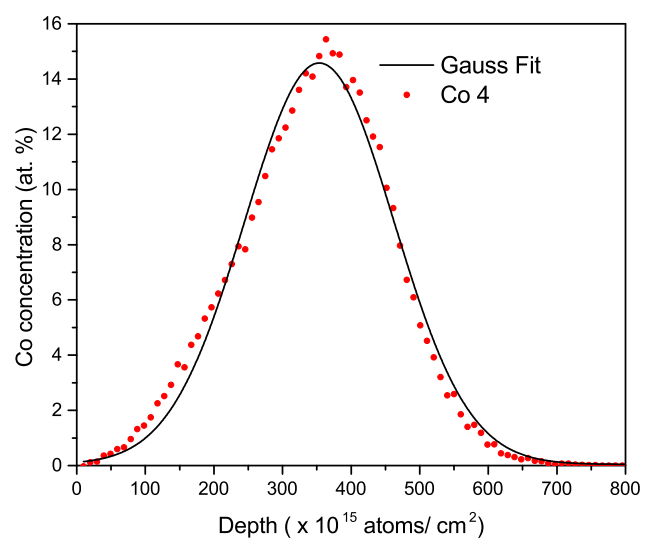

(c) DTRIM prediction of Co depth profile

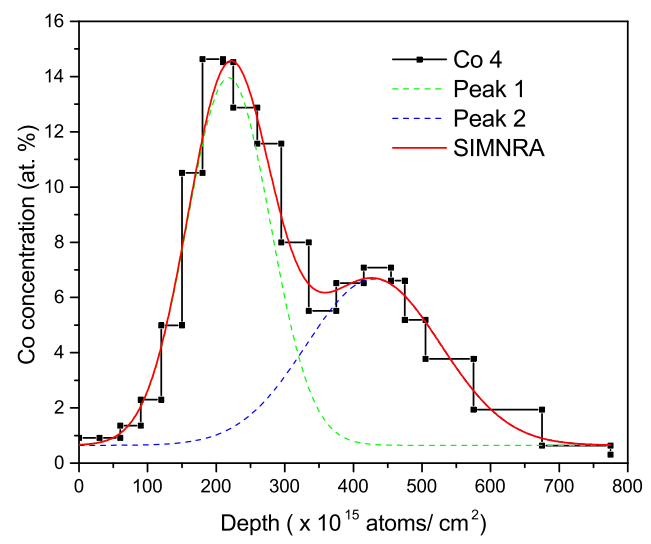

(b) SIMNRA depth profile of Co distribution

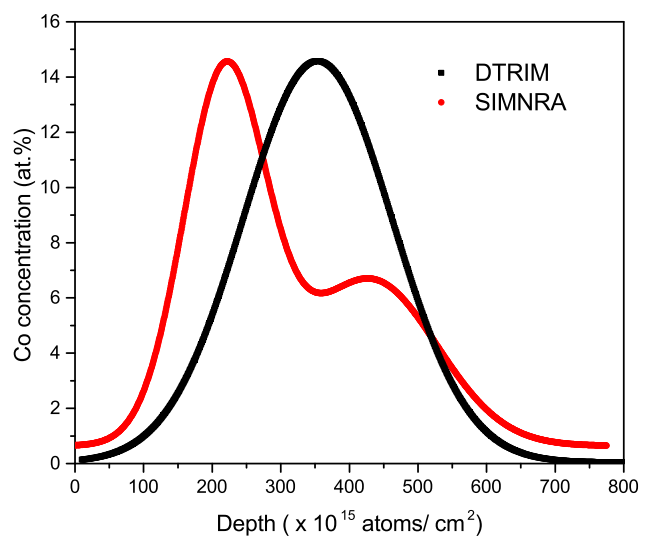

(d) DTRIM Simulation vs Experimental measurement

Figure 5.10: Analysis of HR-RBS spectrum of Co 4. The data analysis reveals preferential displacement of Co towards the surface resulting in an asymmetric bimodal distribution.

Figure 5.10 (a) shows the Co distribution in a counts vs energy plot and the corresponding SIMNRA Co depth profile is shown in figure 5.10 (b). The magnitude of difference between the surface peak concentration and the center peak concentration are clearly visible in these plots. The surface peak corresponds to a Co concentration of 14.62 at.\% extending from 180 to $210 \times 10^{15}$ atoms.cm ${ }^{-2}$. The minima is located in between $335-375 \times 10^{15}$ atoms.cm ${ }^{-2}$ with 5.51 at.\% of Co. This is followed by the center peak at $415-455 \times 10^{15}$ atoms.cm $^{-2}$ with a Co concentration of 7.08 at.\% which is less than half that of the surface peak concentration. The next observation is the presence of Co at the surface to a concentration 0.91 at.\%. As observed for Co 2.4, a similar surface O peak is observed in the HR-RBS spectrum of Co 4 shown in figure 5.10. This as inferred earlier can be attributed to the oxide formation arising from the surface presence of Co. The Co depth distribution is fit with a multi-peak Gaussian 
distribution for the purpose of comparison and analysis. The fit parameters are described in table 5.12.

Table 5.12: Co 4 Experiment vs Theory

\begin{tabular}{|c|c|c|c|c|}
\hline Parameters & Units & Experiment & Experiment & Theory \\
\hline & & Peak 1 & Peak 2 & \\
\hline$y_{0}$ & at.\% & 0.60 & 0.60 & 0.04 \\
\hline$A$ & $\times 10^{15}$ atoms.cm ${ }^{-2}$ & 2023 & 1496 & 3958 \\
\hline$w$ & $\times 10^{15}$ atoms.cm ${ }^{-2}$ & 121 & 197 & 217 \\
\hline$x_{c}$ & $\times 10^{15}$ atoms.cm ${ }^{-2}$ & 218 & 430 & 354 \\
\hline Straggle & $\times 10^{15}$ atoms.cm ${ }^{-2}$ & 61 & 98 & 109 \\
\hline FWHM & $\times 10^{15}$ atoms.cm ${ }^{-2}$ & 143 & 232 & 256 \\
\hline Peak Concentration & at. $\%$ & 13.33 & 6.06 & 14.54 \\
\hline
\end{tabular}

Figure 5.10 (c) shows the predicted Co distribution fit with a Gaussian distribution. For the first time, the predicted distribution is observed to be slightly skewed towards the surface. This can be attributed to the increase in stopping power of the target due to incorporation of significant amount of Co into it. The distribution peaks at $354 \times 10^{15}$ atoms.cm $^{-2}$ with a straggling of $109 \times 10^{15}$ atoms.cm ${ }^{-2}$. The fit from the measured distribution and the predicted distribution are compared in figure 5.10 (d). The comparison yields several differences which are already discussed in detail in the previous section. However, the comparison drawn in figure 5.10 (d) gives insight about the trend the measured distribution is expected to take compared to the predicted distribution. Few of the interesting aspects are, the surface peak concentration is now equal to the Co concentration present at the peak of the DTRIM predicted distribution. The Co accumulated outside the predicted region now comprises $25 \%\left(0.98 \times 10^{16}\right.$ atoms.cm $\left.{ }^{-2}\right)$ of the total dose. Finally the surface peak is further away from the mean projected range than observed before. It is about $150 \times 10^{15}$ atoms.cm $^{-2}$ closer to the surface than the mean projected range. This is a crucial information. The increase in the distance between the surface peak and the mean projected range indicates the effect of increase in fluence. In other words, as the implantation fluence increases it can be expected that the surface peak will move closer towards the surface and will accumulate higher concentration (relative to center peak) than observed so far. The next sample to be investigated confirms this trend.

\subsubsection{High fluence: Enhanced bimodal distribution}

Co 7 is the fourth sample in series. The Co implantation fluence is increased from 4 $\times 10^{16}$ atoms.cm ${ }^{-2}$ to $7 \times 10^{16}$ atoms.cm ${ }^{-2}$. In the last few sections, the distribution of Co 
in DLC at varying fluences were presented. At each implantation fluence, the distribution measured were different. It is of course expected, that with increasing fluence DLC has to accommodate higher Co dose. However, the changes in distribution from one fluence to the other resulted in features that could not be explained or predicted. For example, Co distribution in Co 1.2 almost aligned with the predicted distribution with a minor difference in its straggling. However, Co 2.4 showed a distinct difference from the predicted distribution; Co distribution was bimodal in nature leading to surface and center peaks. In retrospect that minor difference observed in Co 1.2 is inferred to be real and is considered to mark the onset of the bimodal distribution. As the fluence further increased to $4 \times 10^{16}$ atoms.cm ${ }^{-2}$, the surface peak gained prominence. It was observed to have moved closer to the surface and it accumulated nearly twice the Co concentration as that of the center peak. These observations hints a possible trend that is being followed by the Co distribution. As the fluence increases, the surface peak gains more Co atoms and moves closer to the surface. Co distribution measured from Co 7 confirms this prediction. The results will be analyzed in comparison to results obtained from Co 4 sample to provide a better perspective.

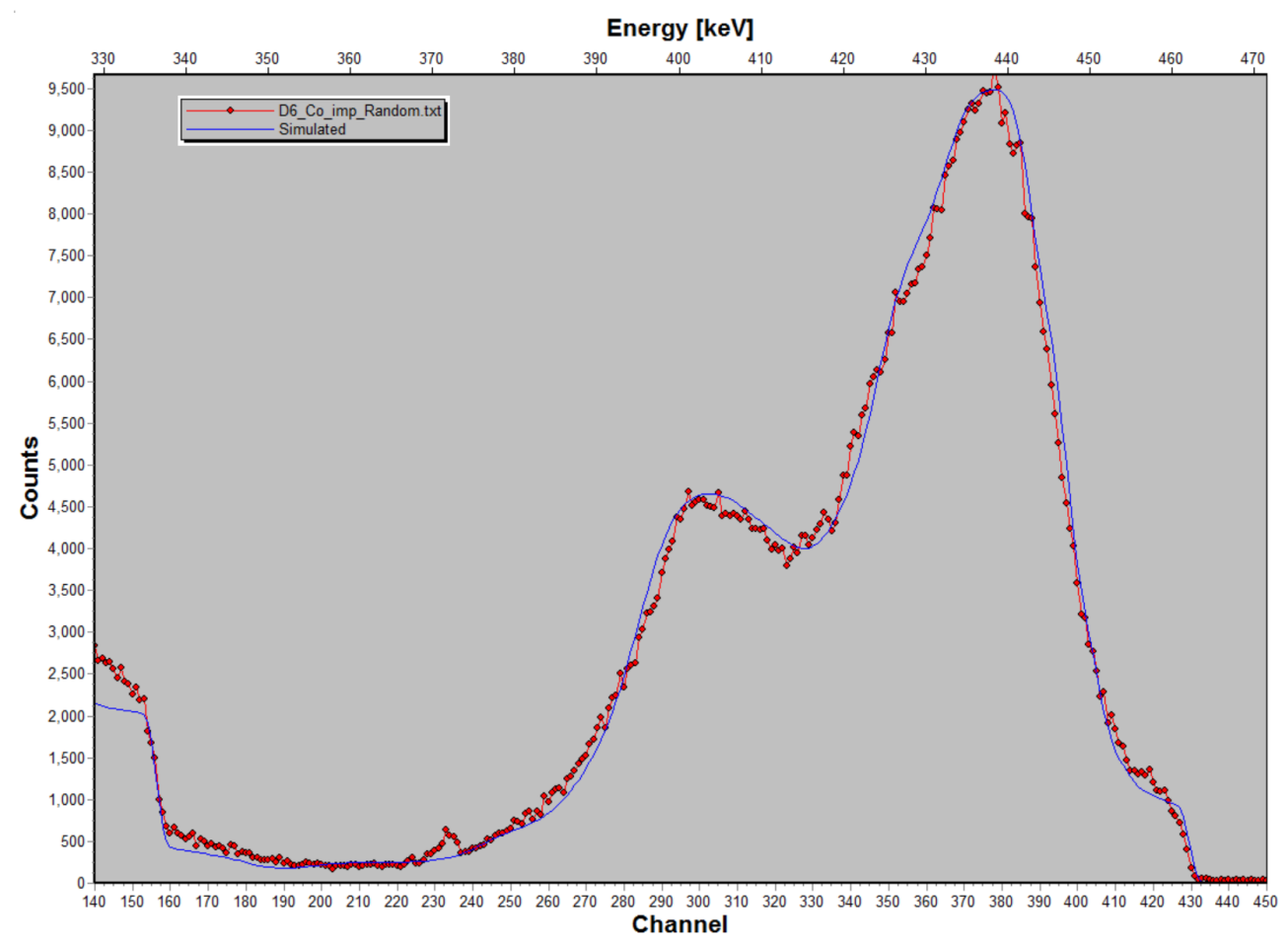

Figure 5.11: SIMNRA fitting of Co 7 HR-RBS spectrum measuring the enhanced bimodal Co distribution in DLC. 
Table 5.13: Co 7 SIMNRA Target composition

\begin{tabular}{|c|c|}
\hline Element & First attempt $\left(\times 10^{15}\right.$ atoms.cm $\left.^{-2}\right)$ \\
\hline \hline $\mathrm{C}$ & 499 \\
\hline $\mathrm{H}$ & 165 \\
\hline $\mathrm{Co}$ & 73 \\
\hline $\mathrm{Si}$ & 100000 \\
\hline
\end{tabular}

Figure 5.11 shows the HR-RBS spectrum of Co 7 sample analyzed with a SIMNRA fit. The spectrum looks similar to an amplified spectrum of Co 4 sample. The bimodal distribution with a strong surface Co peak is seen in the figure. The spectrum is fit with a SIMNRA target composed of 24 layers with an overall Co dose of $7.3 \times 10^{16}$ atoms.cm ${ }^{-2}$. The target summary is provided in table 5.13. Figure 5.11 shows the analysis of the measured Co distribution. 


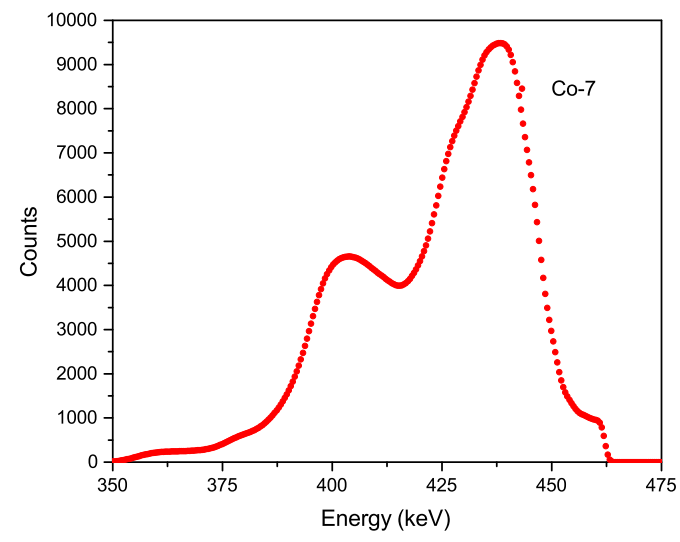

(a) Co profile from HR-RBS spectrum

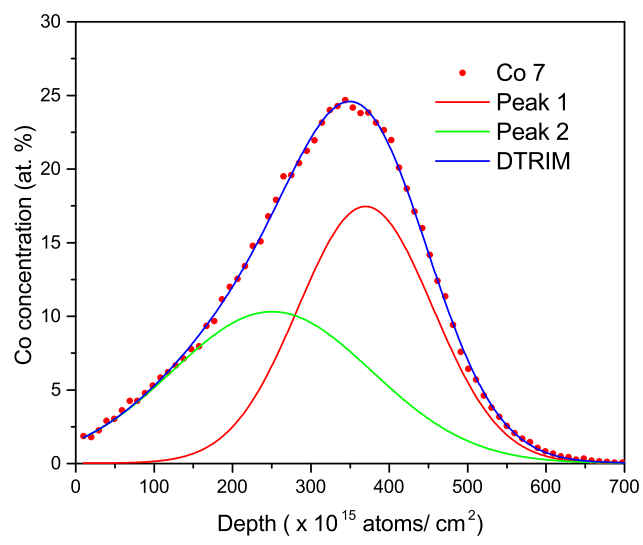

(c) DTRIM prediction of Co depth profile

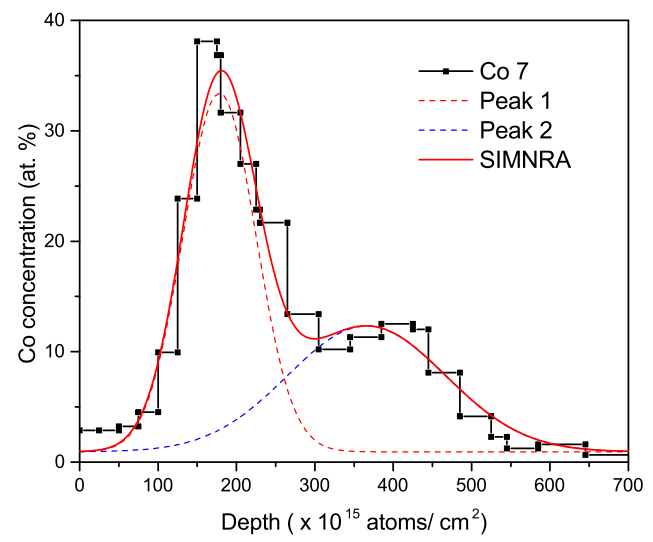

(b) SIMNRA depth profile of Co distribution

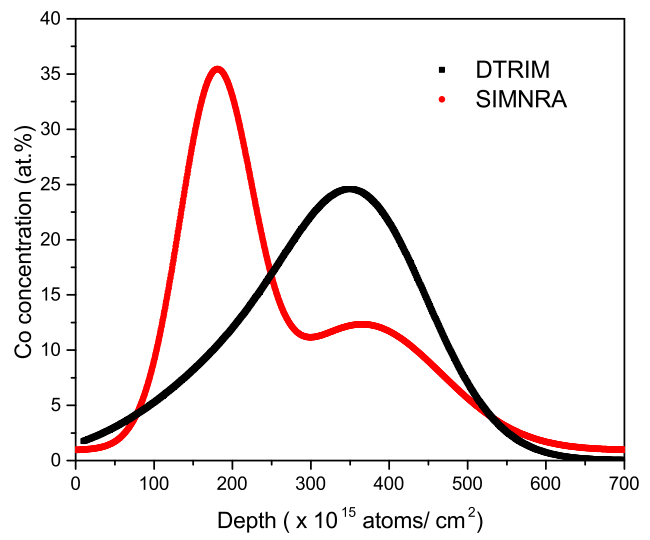

(d) DTRIM Simulation vs Experimental measurement

Figure 5.12: Analysis of HR-RBS spectrum of Co 7. Comparison with DTRIM simulation suggests a major fraction of implanted Co to lie outside the predicted distribution towards the DLC surface.

Figure 5.12 (a) shows the Co distribution in counts vs energy plot and figure 5.12 (b) shows the Co depth distribution of Co 7 sample both extracted from SIMNRA analysis. At the surface, the concentration of Co is measured to be 3.5 at.\%. A corresponding oxygen peak is observed in the HR-RBS spectrum. Following the trend of the Co distribution with increasing fluence, the surface Co peak is observed at $150-175 \times 10^{15}$ atoms.cm $^{-2}$ with a Co concentration of 37.5 at.\%. This is a massive increase in Co concentration when compared to the previous sample. With increasing depth the Co concentration rapidly drops to 10 at. $\%$ at $300 \times 10^{15}$ atoms.cm ${ }^{-2}$. This is followed by a gradual rise in the concentration until reaching the center peak at $425 \times 10^{15}$ atoms. $\mathrm{cm}^{-2}$ with a Co concentration of 12.5 at.\%. The measured distribution is fit with multiple Gaussian peaks and their parameters are shown in table 5.14 . 
Distribution predicted from DTRIM is shown in figure 5.12 (c). The distribution is heavily skewed towards the surface and hence cannot be satisfactorily fit by a single Gaussian distribution. Hence for the purpose of comparison with the measured distribution the predicted distribution is fit with multiple peaks to get a cumulative fit that can in principle be compared to the measured distribution.

Table 5.14: Co 7 Experiment vs Theory

\begin{tabular}{|c|c|c|c|c|c|}
\hline Parameters & Units & Experiment & Experiment & Theory & Theory \\
\hline & & Peak 1 & Peak 2 & Peak 1 & Peak 2 \\
\hline$y_{0}$ & at.\% & 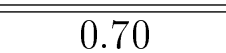 & 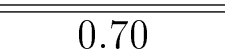 & $\begin{array}{l}0.02 \\
\end{array}$ & 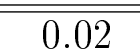 \\
\hline$A$ & $\times 10^{15}$ atoms.cm ${ }^{-2}$ & 3801 & 2976 & 3752 & 3289 \\
\hline$w$ & $\times 10^{15}$ atoms.cm $^{-2}$ & 93 & 204 & 172 & 255 \\
\hline$x_{c}$ & $\times 10^{15}$ atoms.cm $^{-2}$ & 178 & 366 & 370 & 250 \\
\hline Straggle & $\times 10^{15}$ atoms.cm $^{-2}$ & 47 & 102 & 86 & 127 \\
\hline FWHM & $\times 10^{15}$ atoms.cm $^{-2}$ & 110 & 241 & 202 & 300 \\
\hline Peak Concentration & at. $\%$ & 32.55 & 11.62 & 17.44 & 10.29 \\
\hline
\end{tabular}

Figure 5.12 (d) shows the comparison between the measured and the predicted Co distribution. They exhibit similar differences as seen in sample Co 4 and Co 2.4. However, the magnitude of the differences are quite high in this case. The amount of Co atoms lying outside the predicted range constitutes $\sim 30 \%$ of the total dose. The Co concentration at the surface peak is 1.5 times greater than the Co concentration predicted at the mean projected range. These observations confirm the prediction made at the end of last subsection regarding the trend the Co distribution would take with increase in fluence. At this point, it is possible to predict with a greater degree of certainty the near endpoint of bimodal distribution. At a particular fluence, the surface peak will eventually reach the surface and will contain the maximum Co concentration. Accumulation of a large amount of $\mathrm{Co}$ at the surface will result in significant sputtering of Co as inferred from the simulation section 5.2. This will lead to a dynamic equilibrium between the incoming ions and the Co present at the surface. Any increase in implantation fluence will literally have no effect on the Co dose retained. This condition is referred to as steady-state condition. It is obvious that a steady state condition would be achieved for any such implantation. However, the occurrence of such a state is usually expected at a very high fluence. The uniqueness of this case is that the results so far predicts that a steady-state condition can be achieved way before the fluence predicted from DTRIM. The following sub-section illustrates in detail the steady-state condition achieved in this study. 


\subsubsection{Saturation: Steady state condition}

Results so far indicate an early occurrence of steady-state condition with respect to implantation fluence for Co implantation into DLC. The study on ion distribution in DLC can thus be concluded with a sample representing the steady state-condition. For this purpose DLC was implanted with Co fluence of $15 \times 10^{16}$ atoms.cm ${ }^{-2}$ and $20 \times 10^{16}$ atoms.cm ${ }^{-2}$. RBS measurements however showed that both the samples contain same Co implantation dose of $\sim 12 \times 10^{16}$ atoms.cm ${ }^{-2}$. The implantation was repeated to confirm this finding and it was observed that the measurements always yielded a value of $12 \pm 1 \times 10^{16}$ atoms.cm ${ }^{-2}$. The implication of these measurements are that a steady-state condition is already achieved in DLC for a fluence of $\sim 12 \times 10^{16}$ atoms.cm ${ }^{-2}$. Co 12 sample thus represents DLC implanted to $\mathrm{F} \geqslant 12 \times 10^{16}$ atoms.cm ${ }^{-2}$. This work records for the first time in literature that when 30 $\mathrm{keV} \mathrm{Co}^{+}$are implanted into ion beam deposited DLC films, from butane precursor gas with Penning ion source, a steady-state Co profile is reached at a fluence of $12 \times 10^{16}$ atoms.cm ${ }^{-2}$.

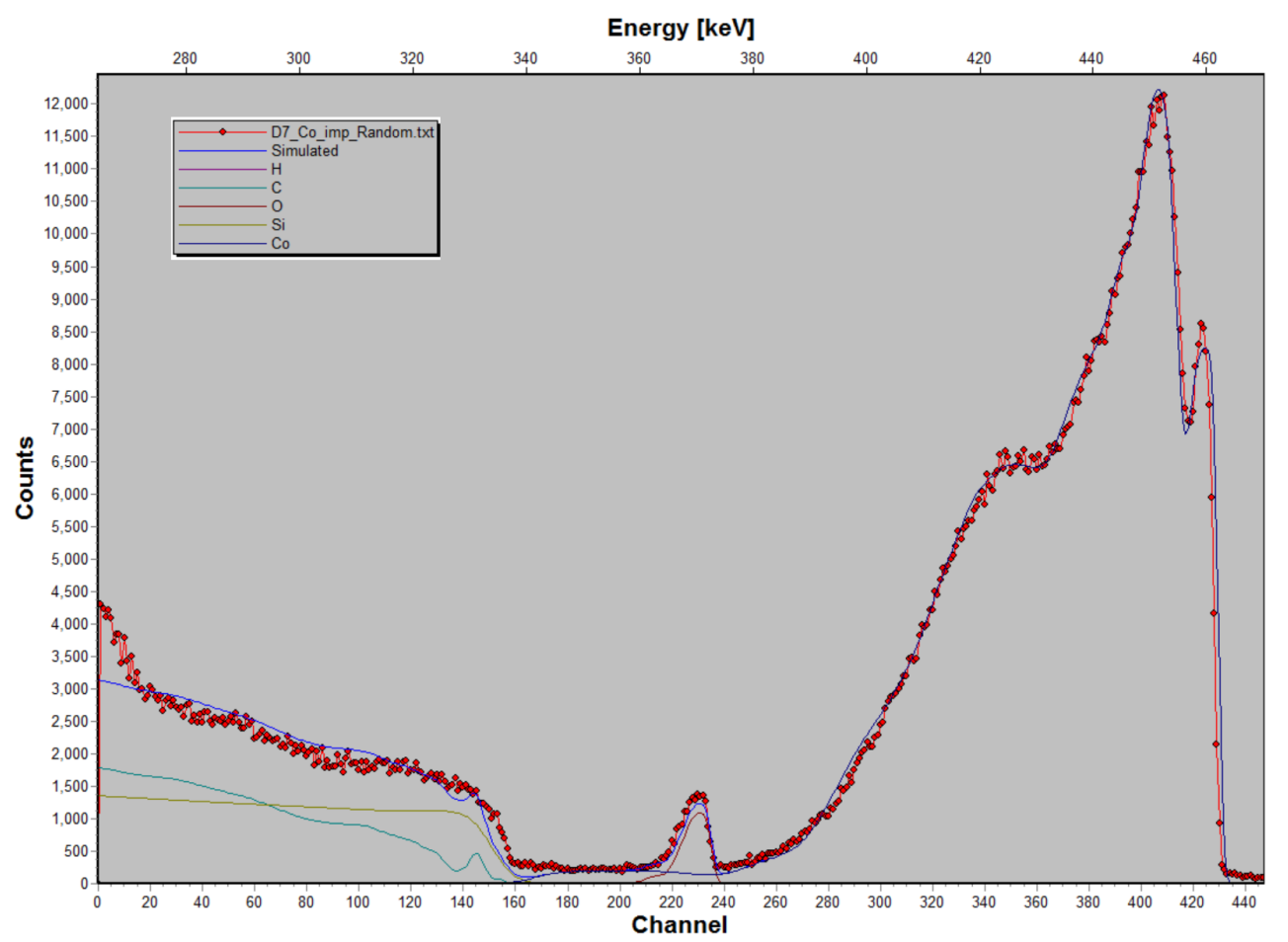

Figure 5.13: SIMNRA fitting of Co 12 HR-RBS spectrum measuring the steady-state distribution of Co implanted into DLC. 
Table 5.15: Co 12 SIMNRA Target composition

\begin{tabular}{|c|c|}
\hline Element & First attempt $\left(\times 10^{15}\right.$ atoms.cm $\left.^{-2}\right)$ \\
\hline \hline $\mathrm{C}$ & 1217 \\
\hline $\mathrm{H}$ & 417 \\
\hline $\mathrm{O}$ & 19 \\
\hline $\mathrm{Co}$ & 107 \\
\hline $\mathrm{Si}$ & 100000 \\
\hline
\end{tabular}

Figure 5.13 shows the HR-RBS spectrum of Co 12 sample fit with SIMNRA. As expected, a large concentration of $\mathrm{Co}$ is accumulated at the surface which oxidizes resulting in the $\mathrm{O}$ peak observed at $373 \mathrm{keV}$. SIMNRA fitting was performed to extract further details from the spectrum. The target summary is displayed in table 5.15. Further analysis on the Co distribution extracted from SIMNRA fitting is shown in figure 5.14. 


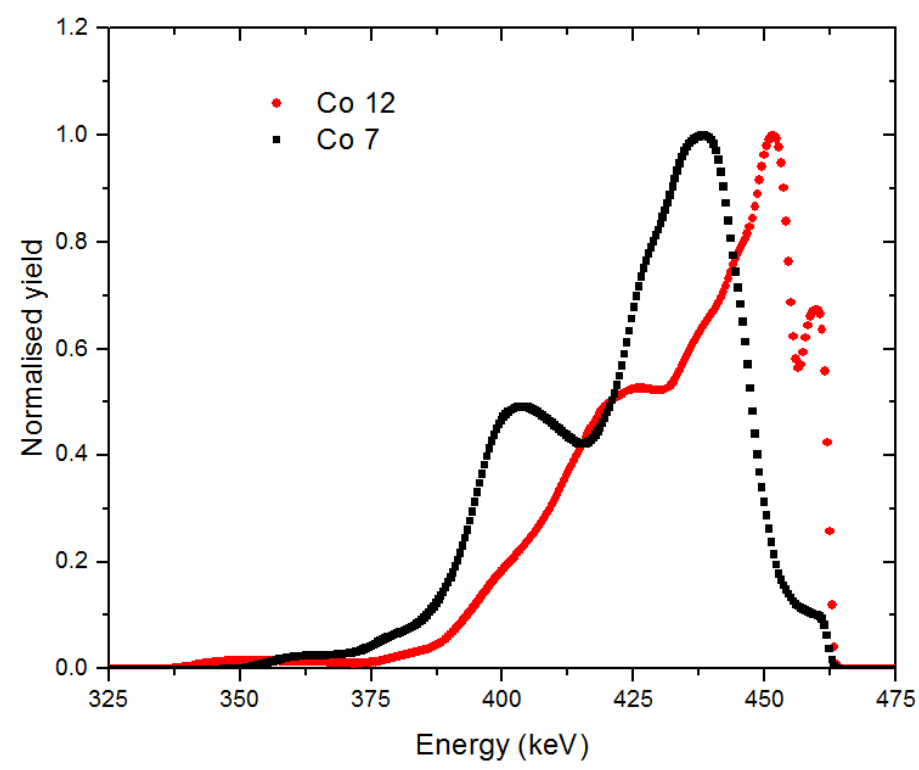

(a) Comparison of Co profile from HR-RBS spectra: Co 12 vs Co 7

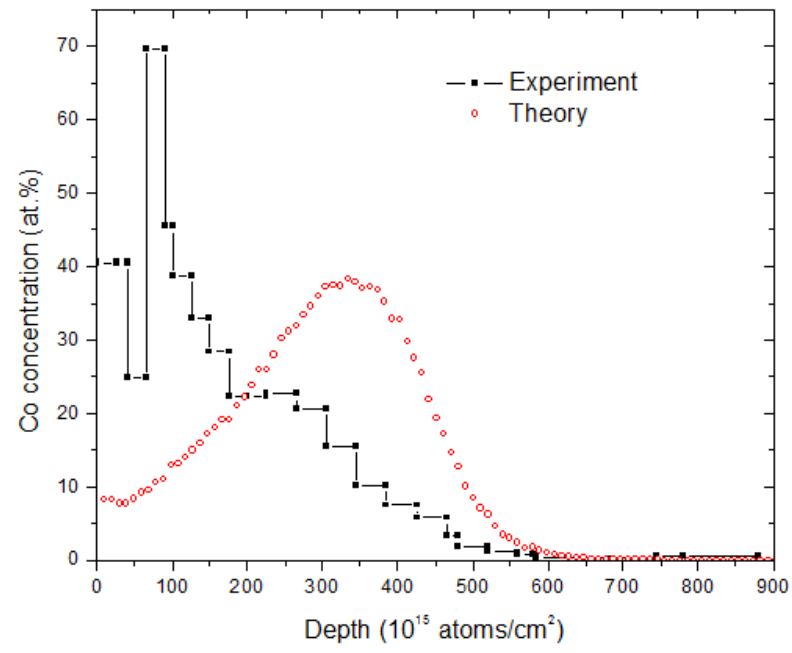

(b) Co 12 Measured distribution vs predicted distribution

Figure 5.14: Analysis of Co distribution in Co 12 in comparison with Co distribution in Co 7 and Co distribution predicted by DTRIM.

Figure 5.14 (a) shows Co distribution of Co 12 compared to Co 7 in energy vs counts plot. The spectrum shows marked differences from the bimodal distribution observed so far. The aim of the analysis is to identify these differences and find possible causes for these 
differences. The highest Co peak observed at $451 \mathrm{keV}$ is the surface peak and the center peak is observed at $426 \mathrm{keV}$. A depleted Co region that is usually observed in between the surface peak and the center peak is not measured in this case. However, a depletion does occur within the surface peak itself leading to a relatively smaller peak at the surface. The same Co distribution represented as depth profile is represented in figure 5.14 (b). The surface layer is almost entirely made of Co and $\mathrm{O}$ with 40 at.\% and 55 at.\% concentration respectively. This layer extend to up to $40 \times 10^{15}$ atoms.cm ${ }^{-2}$. The following depleted layer consists of a Co concentration of up to 25 at.\% which extends from $40-65 \times 10^{15}$ atoms.cm ${ }^{-2}$ This is followed by the original surface Co peak with a maximum Co concentration of up to 70 at.\% at 65 $90 \times 10^{15}$ atoms.cm ${ }^{-2}$. The center peak is observed to have merged with the original surface peak with the second maxima at $225-265 \times 10^{15}$ atoms.cm ${ }^{-2}$ and with a Co concentration of 22.5 at.\%. The depth profile is not fit with multi-peak Gaussian distribution as the observed distribution represents a steady-state condition that involves significant sputtering. The distribution gets skewed to one end and can not best be described by a double Gaussian distribution. Comparison of measured and predicted distribution yields similar information as before. Large amount of Co lies outside the predicted region.

In figure 5.14 (a), the Co distribution in energy scale is compared for samples Co 7 and Co 12. The yield is normalized to 1 so that the differences in the distribution can be better illustrated. First observation is the apparent shift in the peak positions. The trend from earlier results were that the surface peak moves closer to the surface and the center peak moves further away from surface. However, it is seen that both the peaks move towards the surface. It is useful to look back at figure 5.2 (b) to understand the shift better. Figure 5.2 (b) illustrates the shift in the distribution towards the surface due to sputtering. The same effect is responsible for the shift observed in 5.14 (a). The second observation is the filling up of depletion region in between the surface and the center peak. The depletion alternatively is seen within the surface peak leading to an additional sharp peak in the surface. One possible cause could be the shortening of the mean projected range due to nearly a double fold increase in the Co concentration in the near surface region. In order to pinpoint to the specific cause it is however necessary to understand the origin of bimodal distribution.

\subsubsection{Possible causes for bimodality}

HR-RBS measurements so far indicate that the measured Co distribution deviates from the distribution predicted by DTRIM. The extent of deviation seems to be dependent on the implantation fluence. It is now worthwhile to investigate the possible factors responsible for this effect. 
A fundamental experimental factor that can lead to a bimodal distribution of implanted atoms is changes in the implantation energy. If the acceleration voltage applied during the course of implantation was drastically reduced, approximately by $10 \mathrm{kV}$, the projected range of Co ions will decrease from $36 \mathrm{~nm}$ to about $25 \mathrm{~nm}$. However, the use of electromagnet for mass separation eliminates such a possibility since ions of lower energy will not be allowed to pass to the target stage.

Another possible cause for bimodal distribution could be due to charging of the sample surface. Since DLC is insulating it is easy for charges to be accumulated in the film. A significant build up of charges at DLC surface can decelerate the incoming Co ions thereby reducing their projected range. This in turn could lead to a bimodal distribution. Use of high current density is known to cause such charging effects as well [16]. The implanter setup at GNS Science contains an electron gun inside the sample chamber. This electron gun is used to flood the sample continuously with electrons throughout the implantation process to prevent such charging effects from taking place. In order to be completely certain, DLC was covered by a tantalum mask and was implanted with Co at a low current density of $0.2 \mu \mathrm{A} . \mathrm{cm}^{-2}$. The sample was implanted to a fluence of $3.6 \times 10^{16}$ atoms.cm ${ }^{-2}$. HR-RBS measurement showed that reducing the current density or use of a metallic mask during implantation does not prevent the bimodal distribution from happening. In fact, the measured bimodal Co distribution corresponds well with the series of Co implanted samples discussed so far. This eliminates charging as the possible cause for the bimodal distribution.

If experimental error or uncertainty is not the source of the observed bimodal distribution, then there is a more fundamental physical phenomenon at play which is not accounted by the DTRIM simulations. Since DTRIM considers all the effects of ballistics, the bimodality should emerge from its limitations such as,

1. Dynamic changes in the density

2. Precipitation and nucleation

\section{Chemical potential and diffusion effects}

Involvement of any of the above effects to such a significant extent as to transform a unimodal to bimodal distribution justifies the motivation of this research work. In order to understand the process better it is necessary to bring in another perspective. For this purpose we move on to the next technique. 


\subsection{Nanoparticle Formation}

In the previous section, energetic $\mathrm{He}^{+}$ions were used to probe the distribution of Co inside Co implanted DLC. The measurement is based on the scattering of $\mathrm{He}^{+}$ions upon direct collisions with the atoms of the sample. Depending on the number of scattered $\mathrm{He}^{+}$ions at a scattering angle and energy the composition and distribution of different elements, primarily Co, was measured. In this section, the same samples are probed with energetic electrons. Instead of the scattering effect, the technique is based on transmission of electrons through the material. The number of electrons that can be effectively transmitted through a sample region is based on the mass, density and order of the material. In this study TEM was carried out to image the cross-sections of the sample. The aim of this investigation is to obtain information about the distribution and configuration assumed by the implanted ions in the DLC matrix. 


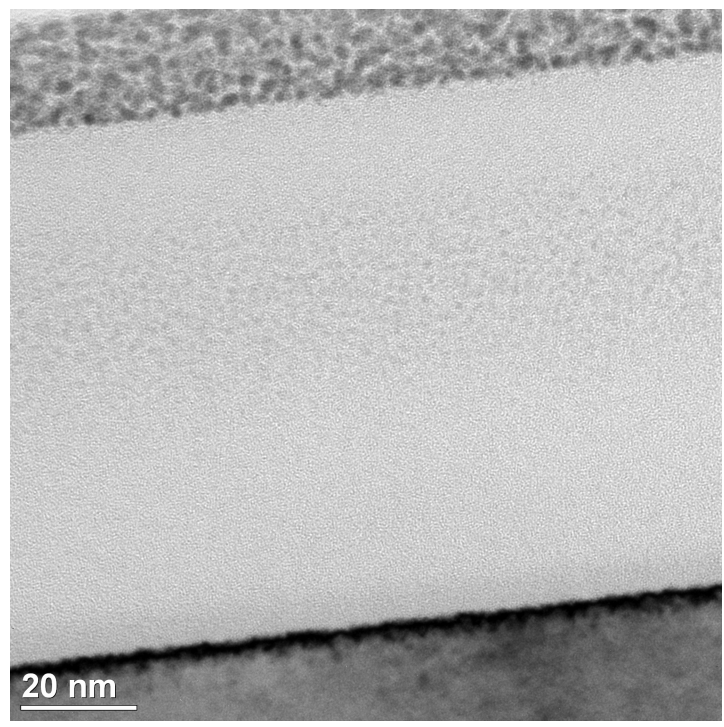

(a) Co 1.2

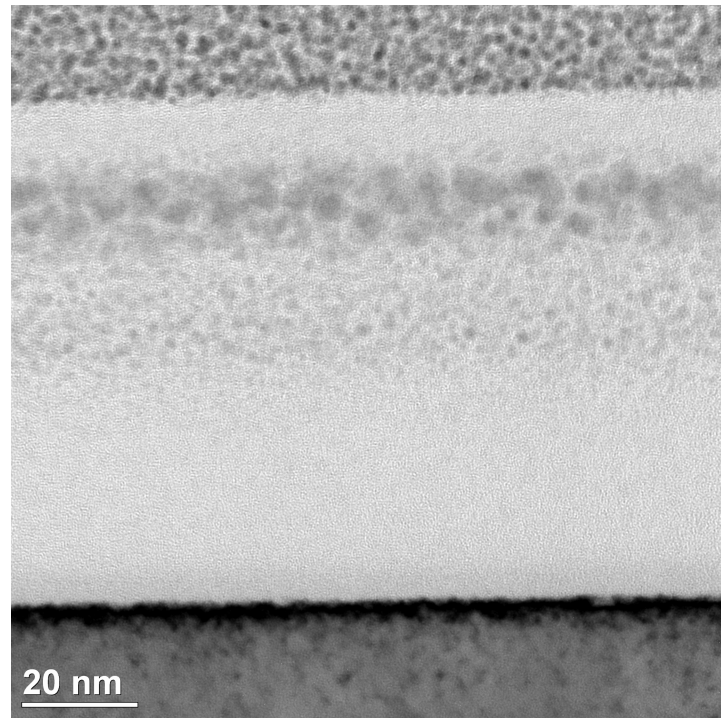

(c) $\mathrm{Co} 7$

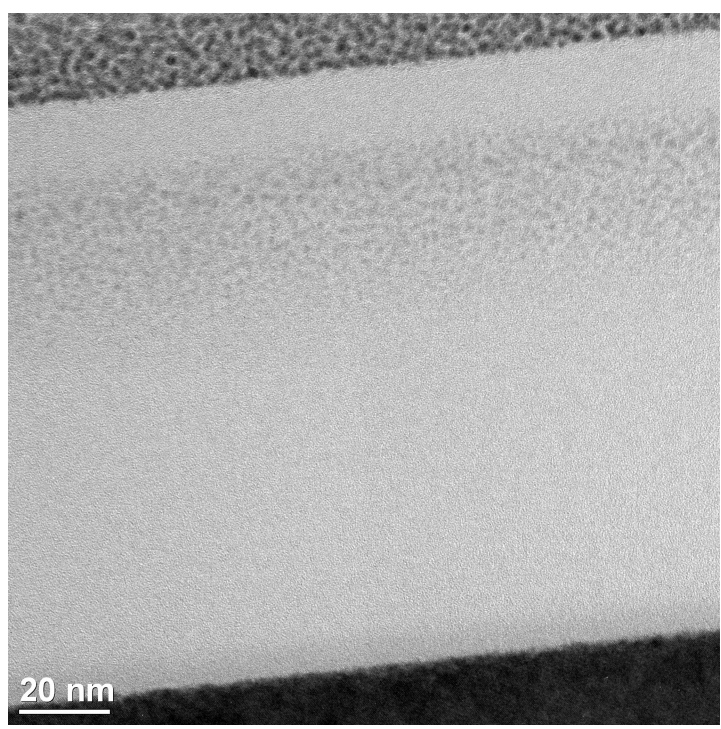

(b) Co 2.4

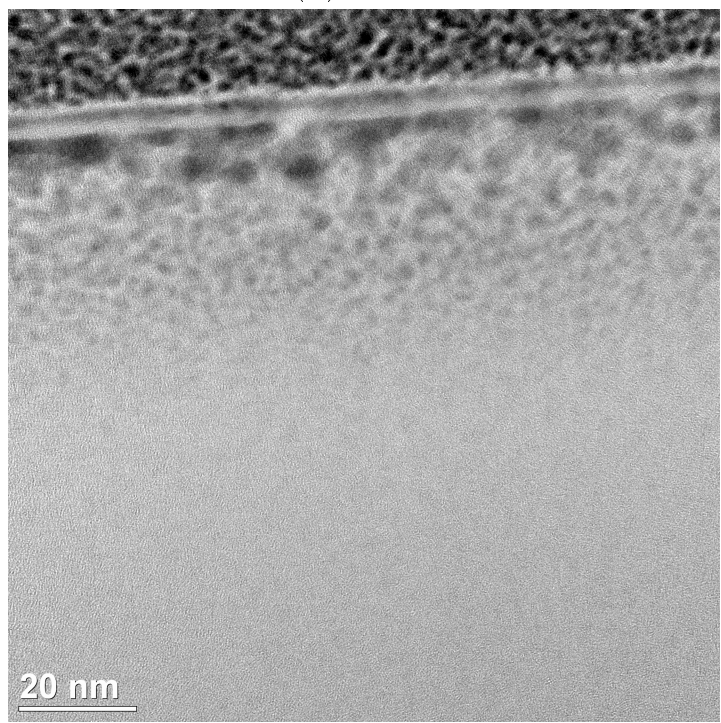

(d) Co 12

Figure 5.15: Cross-sectional TEM images of Co implanted DLC showing the evolution of Co distribution and nanoparticle formation with increasing Co implantation fluence in DLC. Right at top of the image is the platinum layer followed by the DLC surface incorporating the implanted Co nanostructures followed by the unimplanted region and the Si interface.

Figure 5.15 shows the cross-sectional TEM images of sample Co 1.2, Co 2.4, Co 7 and Co 12. As-deposited DLC was already probed in chapter 4. DLC was observed to be completely amorphous with an intermixing layer of $\mathrm{C}, \mathrm{H}$ and $\mathrm{Si}$ at the interface. The surface of the DLC is coated by a platinum layer to prevent damage during sample preparation and to mediate charge conduction and dissipation. The first observation about the implanted DLC films 
is the formation of precipitates within the DLC matrix. Figure 5.15 (a) shows the crosssection of Co 1.2 sample. Small nanoparticles seen as slightly dark circles are observed in the region between 20 - $50 \mathrm{~nm}$ from the sample surface. These nanoparticles can be attributed to the implanted Co atoms. The mechanism behind precipitation can be briefly described as follows. When the concentration of dopant, in this case cobalt, exceeds the solubility limit of the solid matrix, it becomes energetically favorable for the dopants to cluster into nanoparticles. The driving force behind this mechanism is the reduction in surface energy of the dopant atoms in the form of nanoparticles as compared to free atoms [94, 15, 95]. Other possible causes could be the chemical interactions of the dopant atoms with the base matrix [94].

For the sample, Co 1.2, the nanoparticles are characterized by a mean diameter of $1.2 \pm 0.5$ $\mathrm{nm}$. The highest concentration of particles are observed to be at about $35 \mathrm{~nm}$ from the sample surface in agreement with DTRIM predictions. As the implantation fluence increases to $2.4 \times 10^{16}$ atoms.cm ${ }^{-2}$, the concentration of nanoparticles increases especially in the nearsurface region. The average particle size increases to $1.5 \pm 0.7 \mathrm{~nm}$. Since the nanoparticles closer to the surface are slightly larger than those seen at larger depth, the error in the mean particle diameter, which is given as two times of the standard deviation, is slightly larger for this sample. The highest concentration of nanoparticles is observed to be around $24 \mathrm{~nm}$ from the surface matching with the HR-RBS measurement. However, the bimodal distribution of the Co atoms are only distinctly visible for sample Co 7. A dense region near the surface is observed that is accumulated with nanoparticles with a size of $2.9 \pm 1.9 \mathrm{~nm}$. This region represents the surface peak, and is about $16 \mathrm{~nm}$ away from the sample surface. This is followed by a region with a decreased density of nanoparticles. This depletion region in turn is followed by the center peak with a higher density of nanoparticles characterized by a mean diameter of $1.6 \pm 0.7 \mathrm{~nm}$. The center peak is located at a distance of $41 \mathrm{~nm}$ away from the surface. The cross-sectional TEM images correspond well with the RBS profile. It is to be noted that while RBS spectra show Co atoms reaching the surface layer, there are no nanoparticles seen in the first few nanometers of implanted DLC. Although very small nanoparticles in the order of $<1 \mathrm{~nm}$ might be not visible within DLC due to a decreased signal-to-noise ratio, a significant percentage of implanted Co seems to be diluted within the DLC matrix.

The final figure represents Co 12 sample. As expected from HR-RBS measurement, the image exhibits marked variations from the profile observed so far. The surface is covered with a small interconnecting network of Co layer. However, this layer is assumed to be completely oxidized from the HR-RBS measurements. The layer starts from the surface and extends up to $\sim 2-3 \mathrm{~nm}$ from the surface. This layer is briefly followed up by a depleted 
layer lacking Co. The depleted layer extends another $\sim 2 \mathrm{~nm}$ followed by a dense region covered with large nanoparticles. This region corresponds to the large surface peak. As the depth increases the particle size gradually decreases leading to a distribution with a large tail. This corresponds exactly to the distribution measured from HR-RBS confirming the depletion within the surface peak.

\subsubsection{Elemental composition from STEM}

In order to extract maximum information possible from this technique, a particular sample is chosen to do further analysis with the TEM data and facilities. For this purpose, Co 7 sample is chosen. The reason behind the choice is that Co 7 represents the most prominent bimodal distribution and maximum information can thus be gained from this sample. While Co 12 does show interesting features, it is as denoted earlier a special case of steady state distribution. Information obtained from this sample cannot be used to assess the other samples. The first point of analysis is the nanoparticles observed in the TEM image.

There is a technique very closely associated with electron microscopy. This technique commonly referred to as Scanning Transmission Electron Microscopy yields very important information about composition of the sample. Bombardment of energetic electron beam onto the Co implanted DLC results in excitation of electrons of the atoms composing the sample. Excitation of core-shell electrons leads to emission of X-Rays characteristic of the elements present in the material. STEM was performed in two manners: Areal Mapping and Line scan.

Figure 5.16 presents the areal mapping of Co 7. 

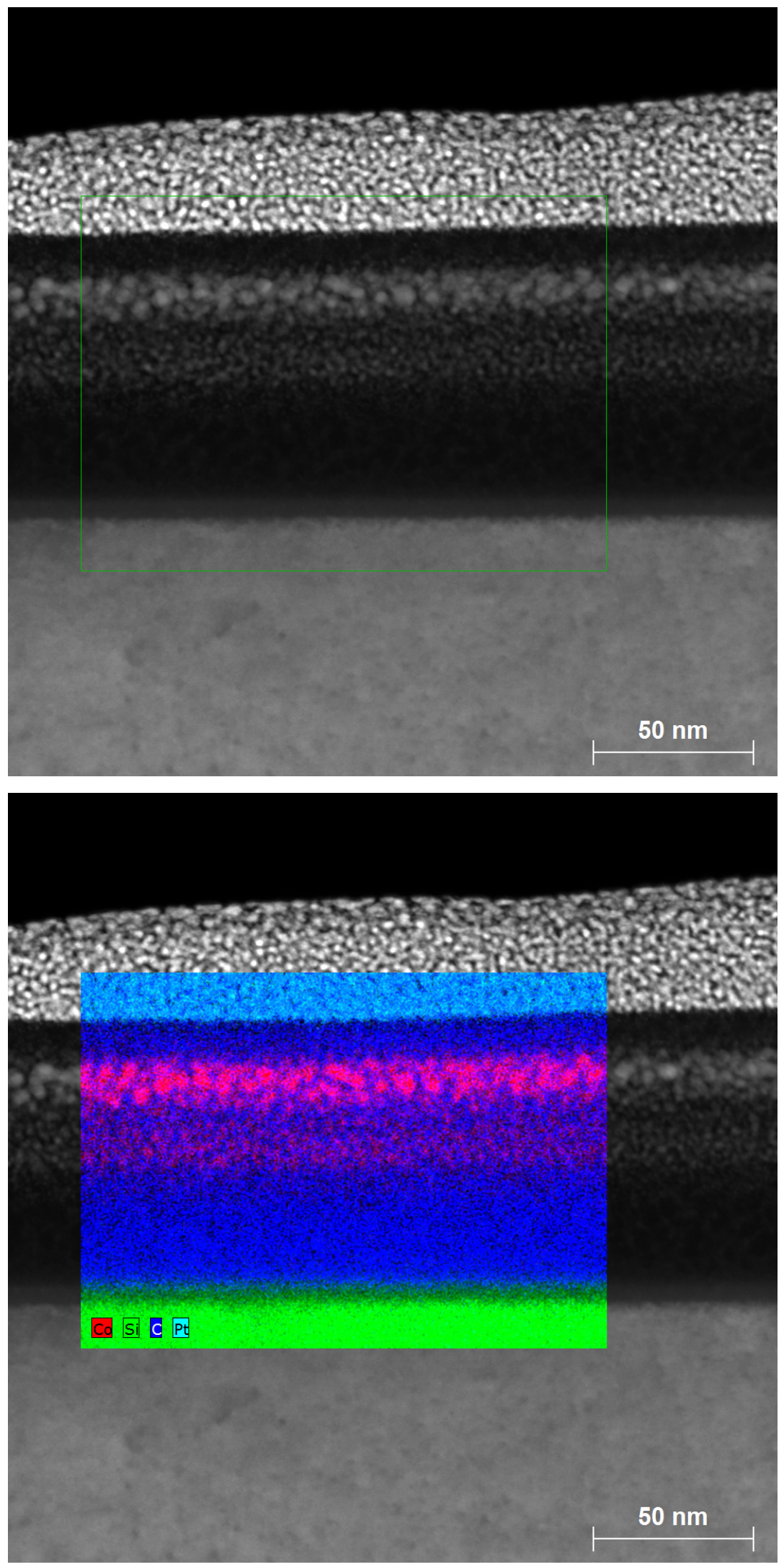

Figure 5.16: Areal compositional mapping of Co 7 TEM cross-section showing the distribution of different elements within the sample. 
In this measurement a particular region of the sample lamella is selected and is continuously raster scanned with the electron beam. The X-rays emitted from the selected area due to interactions with the energetic electrons are collected and analyzed. Each specific wavelength is assigned a particular element based on their unique electronic energy levels. In this case, $\mathrm{K}$ alpha and $\mathrm{K}$ beta lines are considered for $\mathrm{C}, \mathrm{K}$ alpha lines were considered for $\mathrm{Co}$ and $\mathrm{Si}$ and $\mathrm{L}$ alpha lines were considered for Pt. The map clearly shows the Co to be present in nearly two different bands: surface peak and center peak. A large accumulation of Co is seen to be present at the surface peak. The detection limits of this measurement are based on the time of exposure and sample lamella thickness. For this particular case, the detection limits are not good enough to detect the diluted concentration detected by HR-RBS in the surface region. While this gives a visual proof about the arrangement of different elements within the matrix line scan can provide information that is not completely visible in the above map.

Figure 5.17 and 5.18 shows line scans performed on Co 7 sample. 


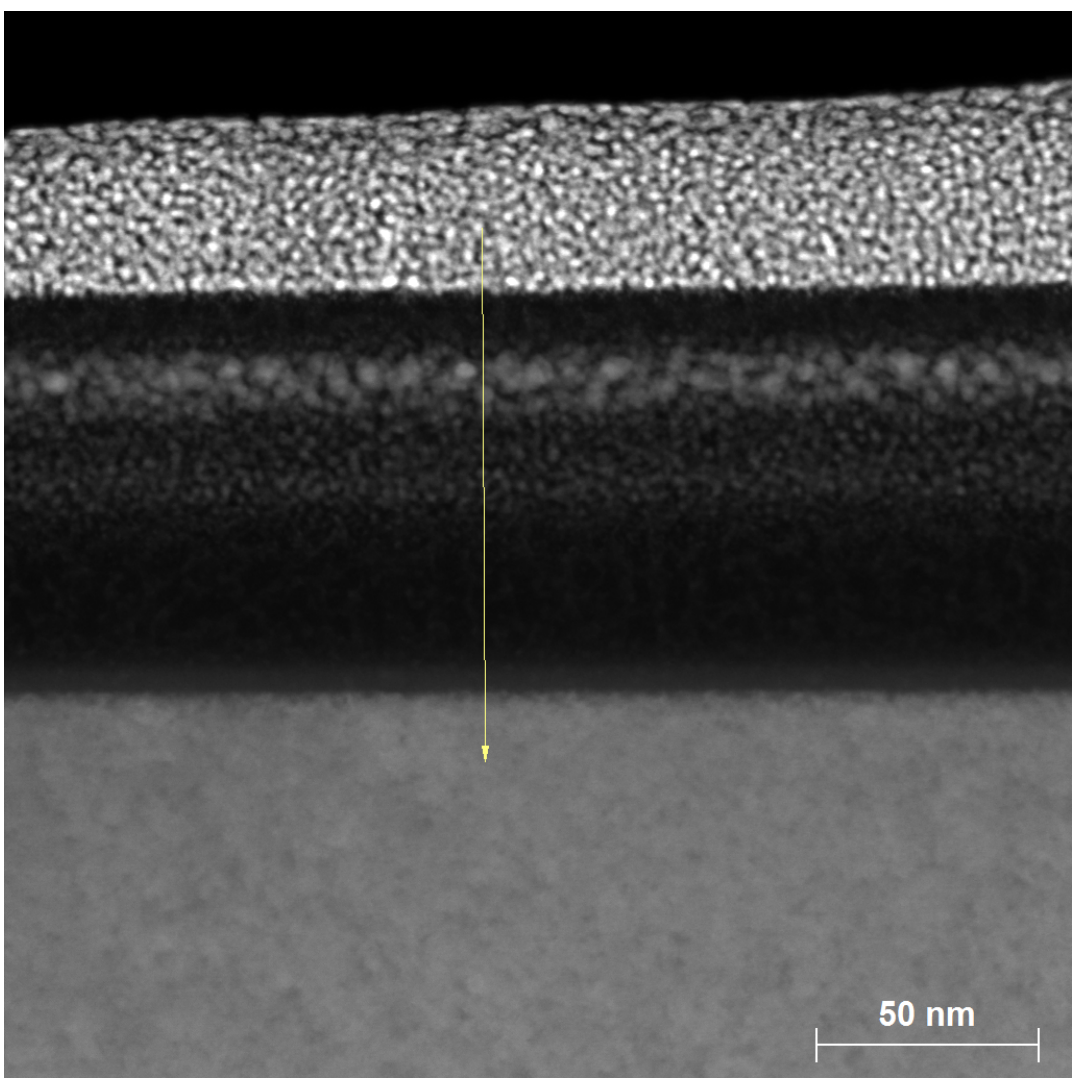

(a) Line scan path

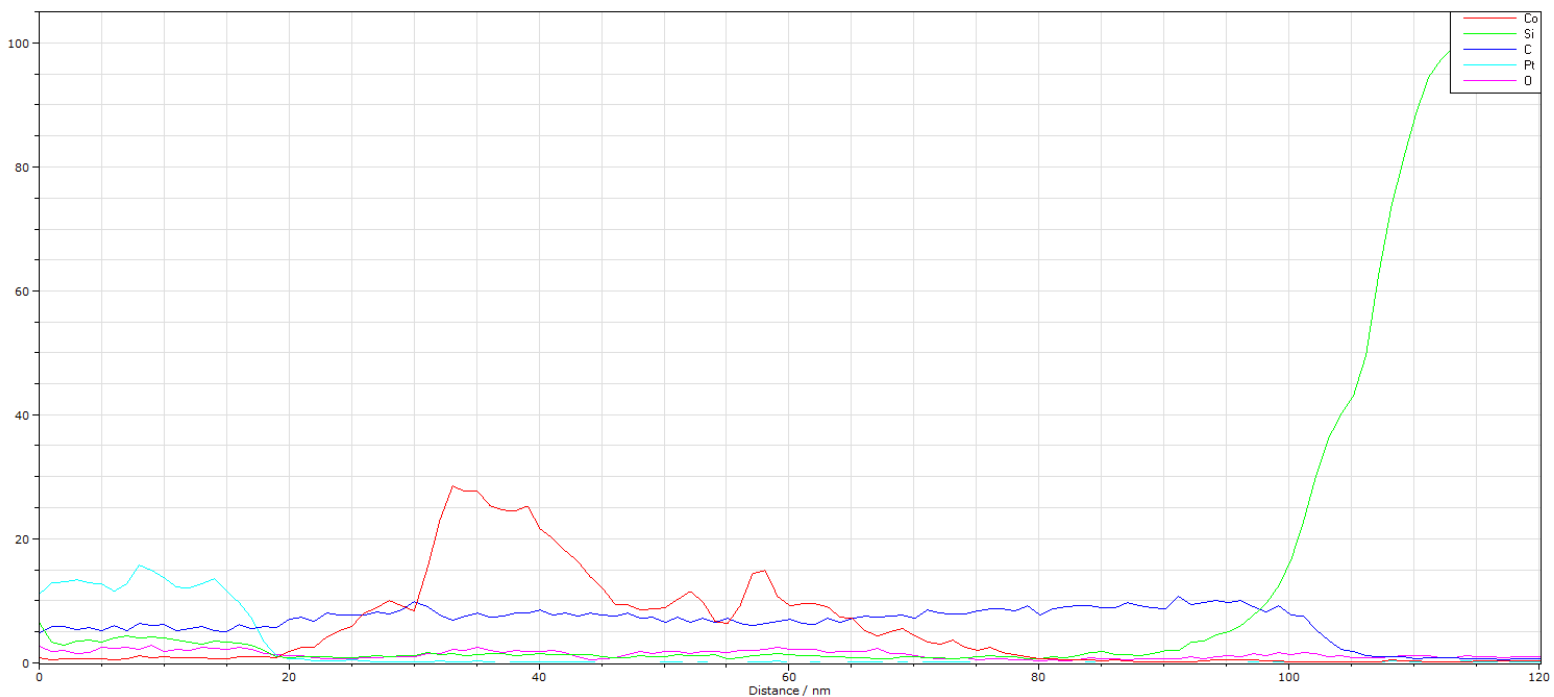

(b) Line scan

Figure 5.17: Line scan 1 of Co implanted DLC showing the distribution of different elements within the TEM lamella. 


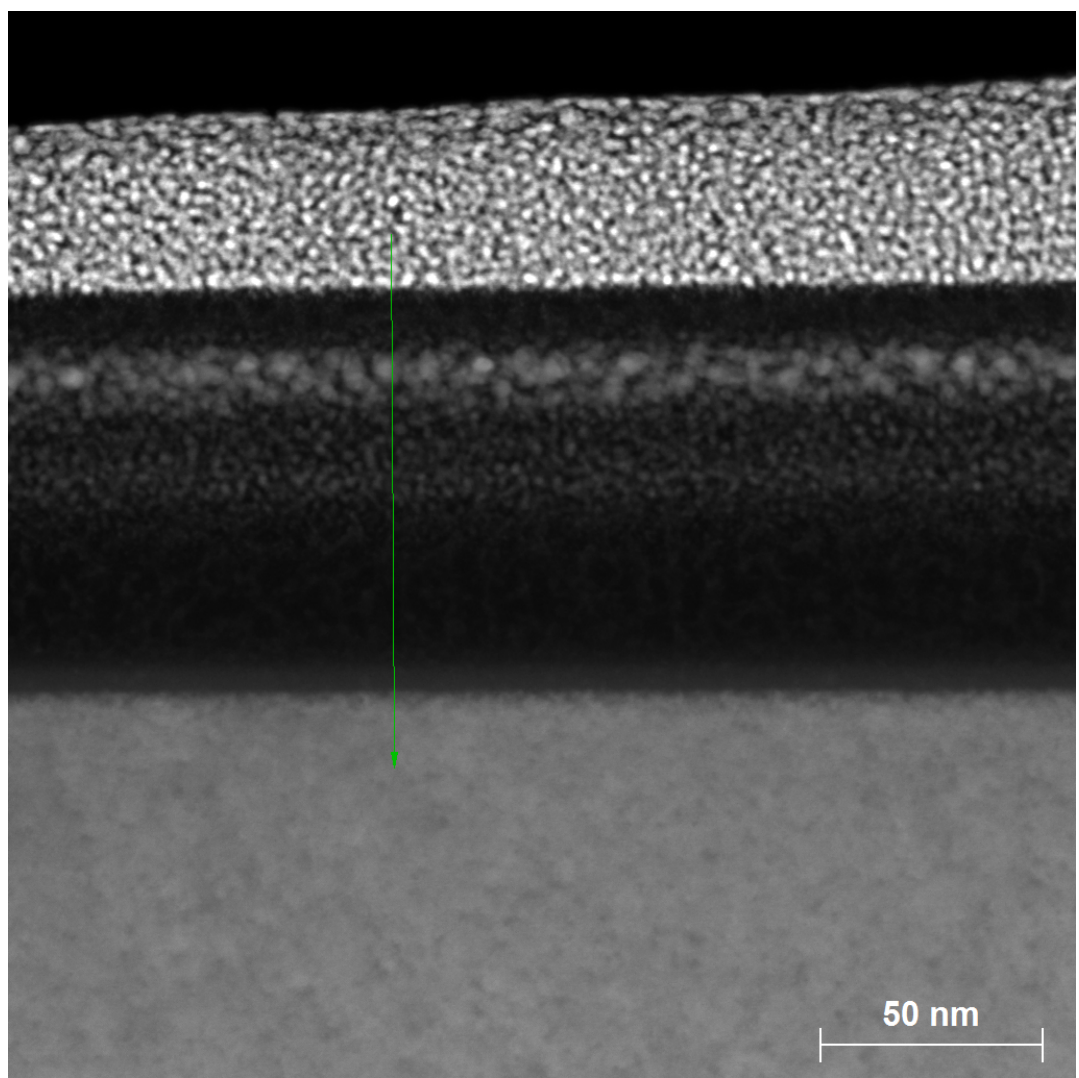

(a) Line scan path

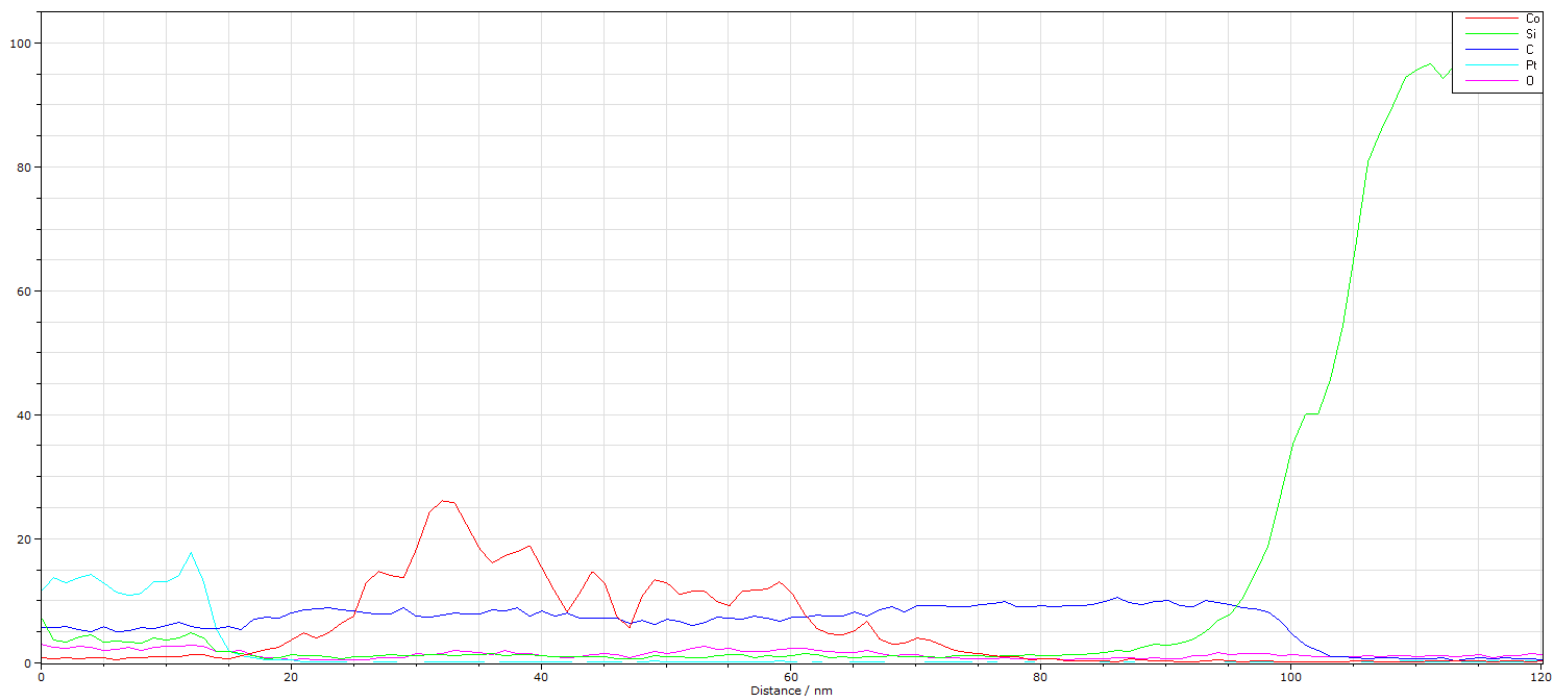

(b) Line scan

Figure 5.18: Line scan 2 of Co implanted DLC showing the distribution of different elements within the TEM lamella.

The recorded line scan represents only a one pixel broad line across the DLC crosssection. This is shown in figure 5.17 (a) along with the corresponding line scan in figure 
5.17 (b). A similar line scan was performed on another region of the sample to compare the uncertainties. It has to be noted that the information represented in the line scan represent the compositional information obtained from a lamella which does not have a uniform thickness due to the nature of FIB preparation. This is an experimental artifact common in FIB prepared sample. The composition thus obtained has to be corrected for the slope of the sample thickness to obtain quantitative information. However, for this study composition values have already been obtained from HR-RBS measurement and this analysis is performed only to compare the obtained results.

Table 5.16: Line scan details - STEM

\begin{tabular}{|c|c|c|c|c|}
\hline Measurement & Surface Peak & Center peak & Depleted & End \\
\hline & Position (nm) & Position (nm) & Position (nm) & Position (nm) \\
\hline \hline Line scan 1 & 14.0 & 39.0 & 36.0 & 61.0 \\
\hline Line scan 2 & 15.8 & 37.8 & 30.9 & 69.7 \\
\hline Contrast Analysis & 15 & 40 & 30 & - \\
\hline
\end{tabular}

The above table provides a very valuable information despite the inaccuracies present in the measurements. The mismatch between both the line scans occur due to the very limited probing region of the measurement. But even if the measurement spans for only a short region it still conforms with our HR-RBS measurement which in comparison to our STEM line scan is a bulk measurement ( $1 \mathrm{~mm}$ beam $=4 \times 10^{6}$ times the line scan width). Another important information confirmed by the line scan is that there is significant amount of Co lying outside the nanoparticles. If you look at the line scans, it can be observed that Co begins as soon as the Pt layer ends confirming that $\mathrm{Co}$ is present at the surface. However, we were unable to see nanoparticles in TEM image in the first $10 \mathrm{~nm}$. This implies that a significant fraction of Co is either diluted or present as nanoparticles with diameter less than $1 \mathrm{~nm}$.

The last row of the table is titled contrast analysis. The TEM picture of Co 7 sample was analyzed using Image $\mathrm{J}$ software to obtain information about the change in brightness of the sample with depth. The result is again strikingly similar to the HR-RBS measurement. 


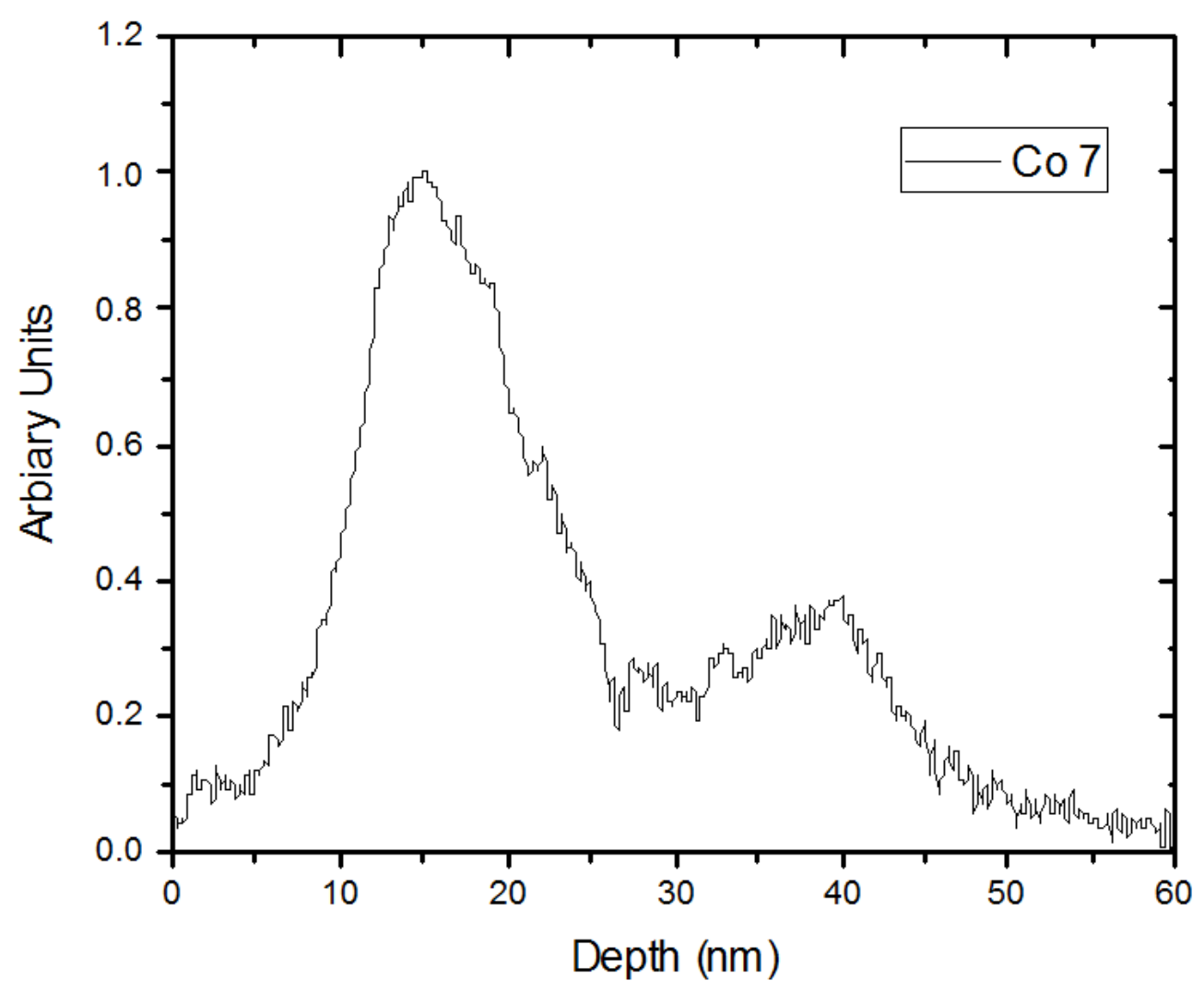

Figure 5.19: Contrast Analysis of the TEM image of Co 7 sample. The contrast analysis reveals the position of the surface and center peak of the bimodal Co distribution.

Figure 5.19 represents the result of contrast analysis performed on the sample. The $\mathrm{X}$ axis represents the depth from the surface and $\mathrm{Y}$ axis contains the contrast information. Peak positions indicates the region with maximum Co. The surface peak position, depleted region and the center peak position can be gathered from this image and are presented in table 5.17. It has to be noted that the same artifact present in the line scan is also relevant for this analysis. From this data, the surface position lies at $15 \mathrm{~nm}$, center peak at $40 \mathrm{~nm}$ and depleted region at $30 \mathrm{~nm}$. This can be directly compared to the Co 7 HR-RBS depth profile. This information is presented in table 5.17. 
Table 5.17: HR-RBS - TEM comparison

\begin{tabular}{|c|c|c|c|}
\hline Regions & TEM & HR-RBS & Density \\
\hline & $\mathrm{nm}$ & $\times 10^{15}$ atoms.cm & $\times 10^{22}$ atoms.cm $^{-3}$ \\
\hline \hline Surface peak & 15 & 162.5 & 10.83 \\
\hline Depleted region & 30 & 325 & 10.83 \\
\hline Predicted peak & 40 & 405 & 10.13 \\
\hline
\end{tabular}

By comparing specific positions at two depth scales, the density of the implanted film up to that position can be calculated. It has to be noted that the density represent an average value up to that point of depth. It can be clearly seen that this average density value in turn changes at different parts of the film. Now that the density value is obtained, what would be its implication on DTRIM measurement? DTRIM uses the same density as that of the DLC film to compute the ion distribution. It has been shown earlier that the peak of the Co distribution is computed to be at $349 \times 10^{15}$ atoms.cm ${ }^{-2}$ and it was originally corresponded to a depth of $35.5 \mathrm{~nm}$ and using the density value described here it would correspond to a depth of $32.2 \mathrm{~nm}$. By not considering the change in density the mean projected range is thus off by $10 \%$. But even more important is the fact that considering the dynamic changes in density alone cannot explain the observed bimodal distribution. As stated, the deviation in mean projected range due to increased density is only $3 \mathrm{~nm}$ however the surface peak is about $20 \mathrm{~nm}$ away from the predicted Co peak.

\subsubsection{Nanoparticle size distribution}

The nanoparticle distribution was described briefly before. In figure 5.20 the complete distribution of the nanoparticles observed for sample Co 7 in figure 5.15 (c) is presented. 


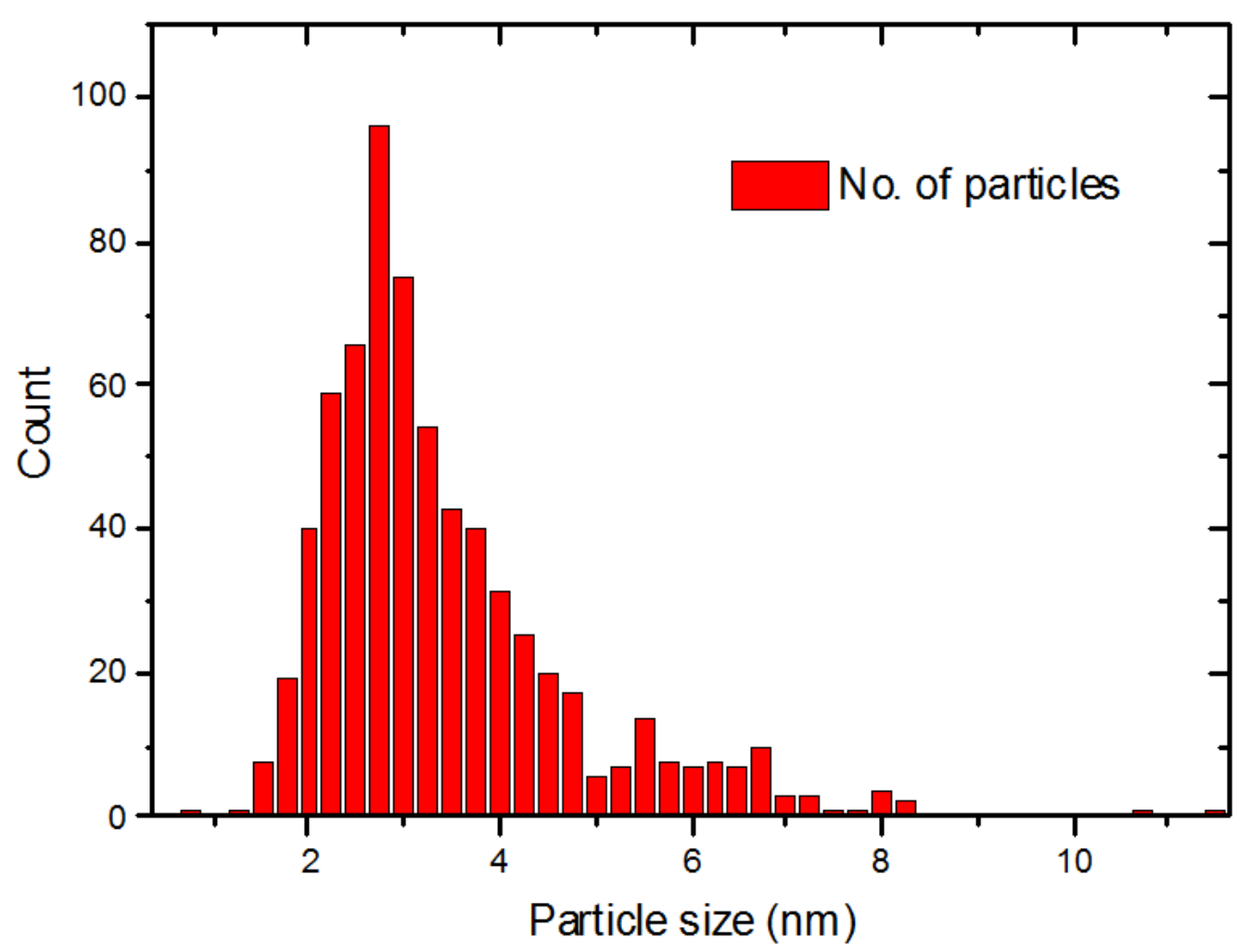

Figure 5.20: Nanoparticle distribution analyzed from TEM image of sample Co 7 shown in figure 5.15 (c). The nanoparticles are observed to be bimodally distributed in size.

As mentioned earlier the TEM analysis is limited to nanoparticles with diameter above $1 \mathrm{~nm}$. It can be seen that about 94 nanoparticles are found with a particle diameter of 2.75 $\mathrm{nm}$. This represents the maximum number of nanoparticles found within the implanted DLC. However, this cannot be taken to represent the maximum Co concentration implanted. The distribution of nanoparticles are also seen to take a bimodal distribution. As the number of nanoparticles drop with increase in size beyond $2.75 \mathrm{~nm}$, there is a small rise in this number at $5.5 \mathrm{~nm}$. Directly comparing this result with TEM suggests that these bigger nanoparticles are observed in the surface peak and the smaller nanoparticles in the center peak.

The next crucial information that can be gathered from TEM images is the crystalline order of the nanoparticles. Nanoparticles present in sample Co 1.2 and Co 2.4 did not exhibit any crystalline order. However DLC implanted with higher fluences show some crystalline order within the large nanoparticles formed in the DLC film. Figure 5.21 shows a representative HR-TEM image of the nanoparticle region of the DLC film implanted with Co to a fluence of $\mathrm{F}=7 \times 10^{16}$ atoms.cm ${ }^{-2}$ together with the corresponding Fast Fourier 
Transform in the inset.

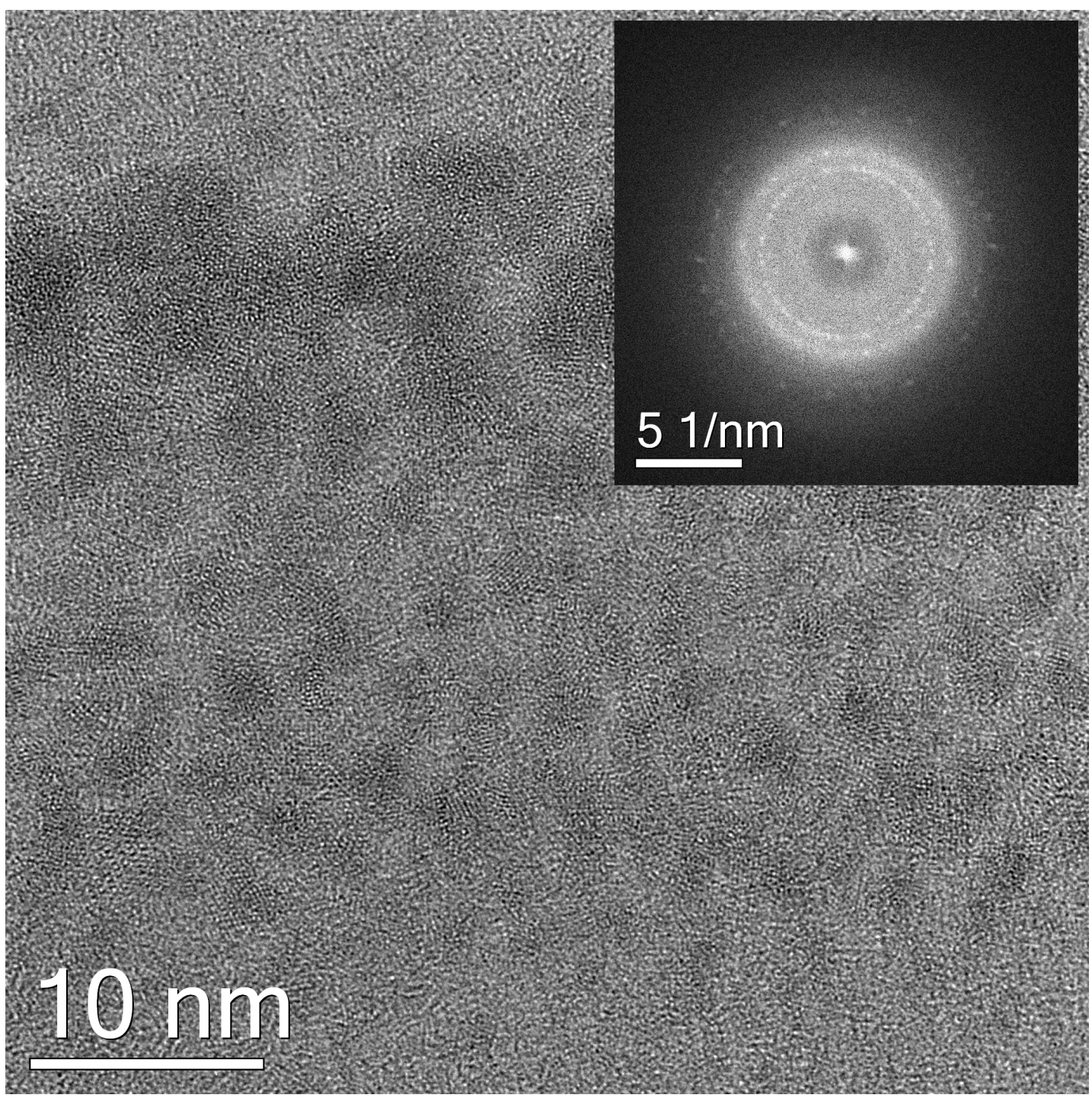

Figure 5.21: HR-TEM image of nanoparticles seen in DLC implanted with a Co fluence of 7 $\times 10^{16}$ atoms.cm ${ }^{-2}$. The inset shows the Fast Fourier Transform of the HR-TEM image.

Here, the ring patterns observed point towards the crystalline nature of the nanoparticles. In particular, distinct d-spacing's of 0.248, 0.208, 0.157, and $0.145 \mathrm{~nm}$ are determined. They do not coincide with those of metallic cobalt in the cubic or hexagonal phases even assuming a slight variation of the lattice parameters caused by local stress. Co hydrides, such as $\mathrm{CoH}_{0.19}, \mathrm{CoH}_{0.26}$, or $\mathrm{CoH}_{0.34}$ can also be excluded. The diffraction rings corresponds closely to forms of Cobalt carbide, $\mathrm{Co}_{2} \mathrm{C}$ or $\mathrm{Co}_{3} \mathrm{C}$. However, the attribution cannot be completely confirmed since a higher number of ring patterns should be visible in the FFT. Thus, while crystalline nanoparticles containing Co are observed, the exact phase and composition cannot be precisely determined. 
The results from TEM investigations can thus be summarized as,

1. TEM confirms the bimodal distribution of implanted Co atoms. At higher fluences this distribution resembles a three banded structure with a dense region with large nanoparticles on the top corresponding to the surface peak. This is followed by a depleted region with a lower density of nanoparticles. This is again followed by a region of small nanoparticles extending deep into the matrix. This region denotes the center peak observed in HR-RBS.

2. Co implantation leads to nanoparticle formation at fluence as low as $1.2 \times 10^{16}$ atoms.cm $^{-2}$. As the implantation fluence increases the nanoparticle gains in size and large nanoparticles are formed in the near-surface region.

3. STEM investigations reveal the distribution of different elements within the matrix. It confirms the presence of Co in the nanoparticles and the line scan further confirms the bimodal nature of the distribution. Contrast analysis on TEM images points towards the position of surface and center peaks in implanted DLC. Comparing the depth in nanometer scale to depth in $\times 10^{15}$ atoms. $\mathrm{cm}^{-2}$ indicates large changes in density within different region of the implanted film.

4. FFT on HR-TEM images was performed on all implanted sample. Only Co 7 and Co 12 showed some ordered patterns. The exact phase and composition of nanoparticles could not be determined from these rings. However, the data resembles closely to that of $\mathrm{Co}_{2} \mathrm{C}$ and $\mathrm{Co}_{3} \mathrm{C}$ indicating chemical interaction of the implanted atoms with DLC matrix.

\subsection{Model - Evolution of bimodality}

HR-RBS and TEM investigations have provided a lot of information about the distribution and configuration of implanted Co atoms within the DLC matrix. In the HR-RBS section, the bimodal distribution of Co in implanted DLC was presented and near the end of the section the possible causes for bimodality were briefly discussed. The investigation eliminated the possibilities of experimental errors to result in such a distribution. It was also mentioned that limitations of DTRIM which is the non consideration of effects such as nanoparticle formation, dynamic changes in density and diffusion effects can possibly lead to the measured bimodal distributions. Further investigations by TEM provide us the missing links needed to fabricate a model explaining the observed distribution. Figure 5.21 summarizes to a large extent the results obtained so far. 


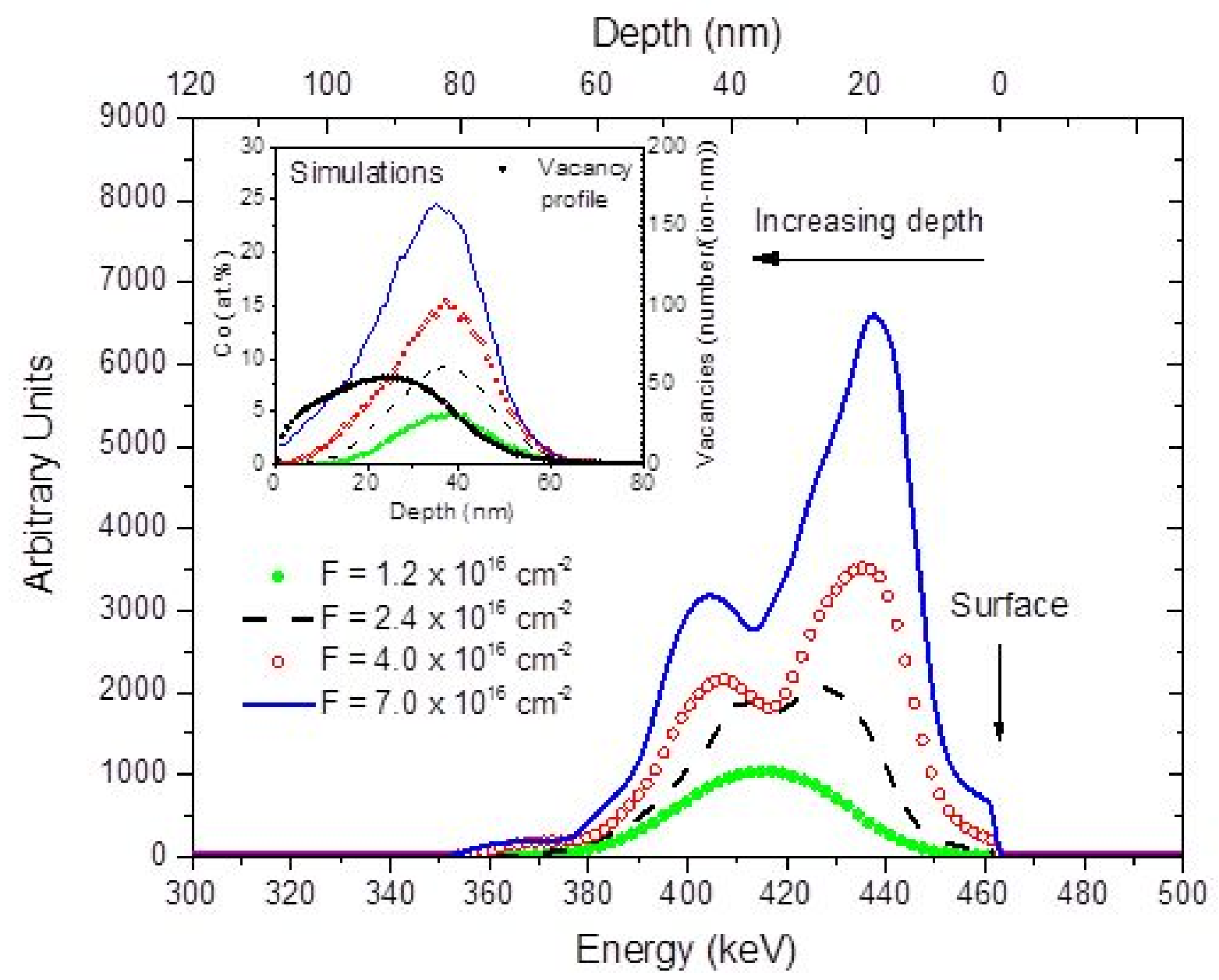

(a) HR-RBS spectra of Co 1.2 to Co 7

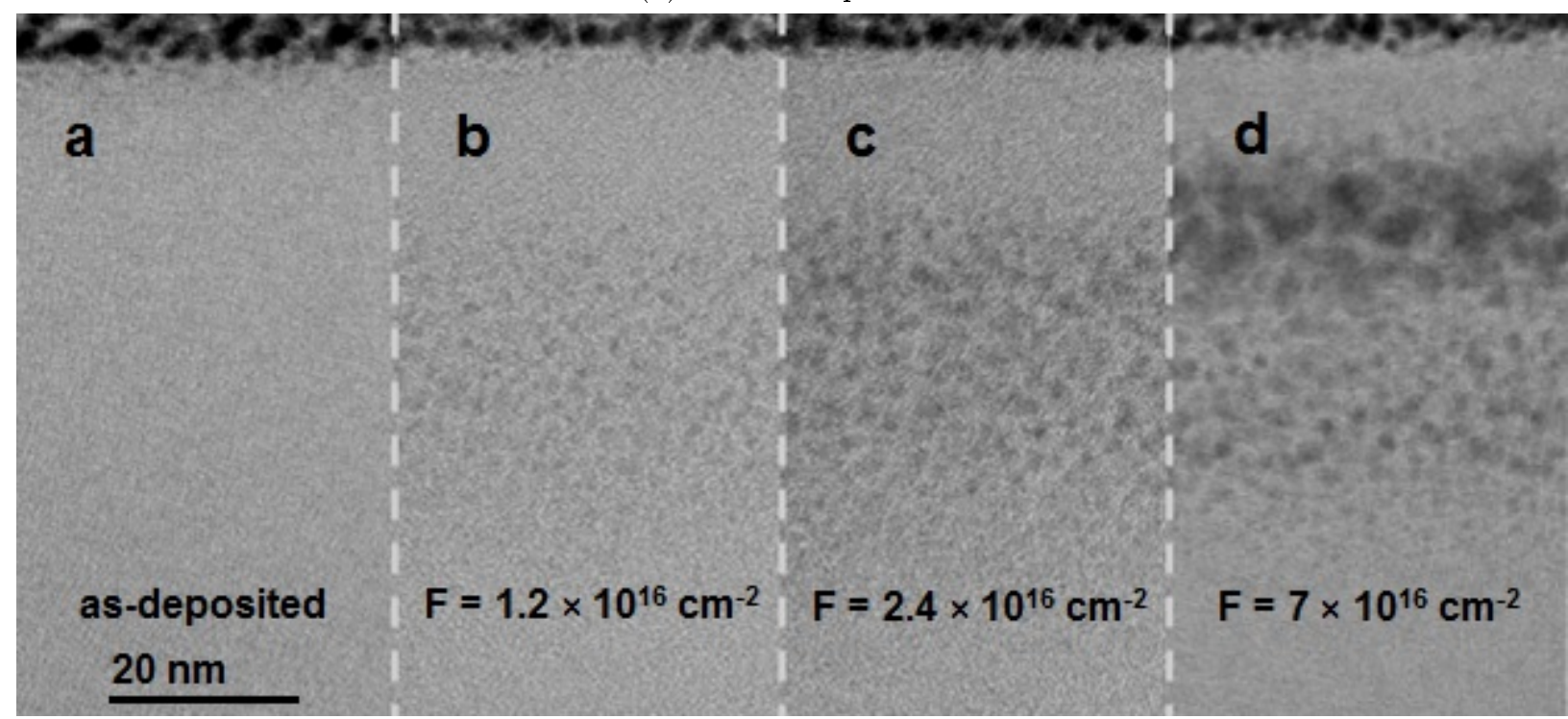

(b) TEM images of Co 1.2 to Co 7

Figure 5.22: Evolution of bimodal distribution in Co implanted DLC evidenced by HR-RBS and TEM measurements. 
TEM investigations revealed the presence of nanoparticles and significant changes in density of the material. This in retrospect is a logical implication of high fluence implantation. It has earlier been stated that mono-energetic ion implantation cannot lead to dual projected ranges. Therefore, it can be concluded that the implanted atoms still accumulate at the projected range but rearrange in the DLC matrix after reaching $R_{p}$ due to other processes. This is evidenced in figure 5.22 (a). As the fluence increases from 1.2 to $2.4 \times 10^{16}$ atoms.cm $^{-2}$, the depletion region at higher fluence coincides with the Co maximum observed at the lowest fluence. The direction of rearrangement suggests that the process is initially driven by the concentration gradient found in the simulated Gaussian profile that involves localized diffusion effects [96].

It is to be noted that mono-energetic ion implantation in general lead to a distribution with concentration gradient. However, for this concentration gradient to result in significant diffusion effects, a considerable amount of energy is required to activate this process. In this case, the implantation is performed at room temperature and thus does not produce significant bulk heating effects ( $30 \mathrm{kV} \times 5 \mu \mathrm{A}=0.15 \mathrm{~W}$ is the power deposited per unit $\mathrm{cm}^{2}$ ). Therefore the diffusion effects could arise only from the energy dissipated by the implanted ions within the DLC matrix by the process of collision cascades and thermal spikes.

Collision cascades occurs in every implantation process. However, hydrogenated DLC is an excellent thermal insulator $\left(0.3 \mathrm{~W} .(\mathrm{mK})^{-1}\right)$ [43]. Thus, unlike other materials, it takes longer for energy release during thermal spikes to be dissipated as heat [13]. This prolonged exposure to 'heat' released during relaxation of thermal spikes enhances localized diffusion of the Co atoms. This hypothesis explains only a part of the picture. Diffusion processes are generally correlated with the broadening of the implanted distribution. Stepanov et al, in his work show the calculated profiles of implanted ion distribution upon diffusion at different implantation energy and temperature [94]. It can clearly be seen that the diffusion does not lead to bimodal distribution However, TEM results suggest presence of another competing factor along with diffusion.

As can be seen in figure 5.22 (b) Co implantation is observed to result in nanoparticle formation. It can be clearly seen from the figure that precipitation is observed at the mean projected range for Co 1.2. As the fluence increases larger nanoparticles are observed to have formed at the near-surface region followed by a depletion region and a region of smaller nanoparticles. The implication of nanoparticle formation for this model is that these nanoparticles act as sinks for the diffusing Co atoms. Once the Co atoms forms these nanoparticles, they do not participate in further diffusion. Thus while overall Co concentration is higher at the near-surface region, the Co atoms that are not bound in nanoparticles and thus available for diffusion is still high at the mean projected range. Thus there ex- 
ists a net diffusion of Co atoms from the projected range to surface even though the Co concentration is higher at the surface than the mean projected range.

A final missing link to this model is the preferential formation of large nanoparticles in the near-surface region compared to the projected range. As mentioned before, literature review suggests that the largest nanoparticles are usually observed at the mean projected range since the concentration of implanted ions are higher at this region. However, this can be prevented in presence of a competing mechanism such as diffusion [94]. Thus, lack of large nanoparticles at the projected range indicates that the diffusion processes dominate at this region preventing formation of large nanoparticles. However this is not true for the entire film. There are regions in the implanted film that acts as catalysts for nanoparticle formation. These are regions where there is a high concentration of defects. It has been proved that defects can act as nucleating centers for nanoparticle growth [1]. In general, ion implantation leads to defect formation with maximum defects concentrated in the region before the mean projected range. The inset in figure 5.22 (a) shows the vacancy peak to be located in the near-surface region before the mean projected range. It is thus not a coincidence that the surface peak is also measured to be located at the same region. Thus it can be inferred that the defects produced during ion implantation in DLC acts as traps for the diffusing Co atoms. These trapped atoms act as nucleating sites for nanoparticle growth. In the presence of energy from the collision cascades these nanoparticles grow into larger nanoparticles at the expense of smaller nanoparticles in a process similar to Ostwald Ripening. While the diffusion processes are still active at this region they are not strong enough to prevent formation of large nanoparticles.

The model can be summarized in the following steps,

1. Ion implantation into DLC leads to unimodal distribution at low fluence.

2. Ion implantation in general results in collision cascades that leads to formation of defects in the near surface region and thermal spikes originating from the projected range.

3. Since DLC is a thermal insulator it takes relatively longer time for heat to be dissipated as thermal spikes.

4. The prolonged exposure to heat and presence of concentration gradient causes diffusion of Co atoms towards the surface (and interface, but since collision cascades are concentrated in the near-surface region only minor concentration of Co diffuse beyond $R_{p}$ ). 
5. Increase in implantation fluence increases the Co concentration and the defect concentration.

6. When the concentration of Co is beyond the solubility limit precipitation occurs.

7. The defects formed during implantation act as traps for the incoming Co atoms leading to formation of nucleating centers.

8. As the Co fluence further increases, these nucleating sites grow into large nanoparticles. These large nanoparticles act as sink for the diffusing Co atoms.

9. At the region near $R_{p}$ the diffusion effects still dominate, preventing formation of large nanoparticles.

10. Since the Co atoms present in the nanoparticle do not participate in the diffusion the concentration gradient is preserved leading to the bimodal distribution observed.

The model is based on the following hypothesis,

1. Slower heat dissipation from thermal spikes leading to diffusion.

2. Precipitation and diffusion acting as competing forces determining formation of nanoparticles.

3. Defects acting as preferential sites for nanoparticle formation.

The formation of nanoparticles at the same site where maximum defects are concentrated supports hypothesis 3 . There is ample evidence in literature to support and prove hypothesis 2. The review by Stepanov [94] on synthesis of nanoparticles by ion implantation clearly mentions the effect of diffusion for nanoparticle formation. Hypothesis 1 is the crucial part that is harder to prove.

If hypothesis 1 is true then similar effects should be observed in other thermal insulators. However, no such effects were observed in other thermal insulators for the implantation conditions used in this research. But a more thorough investigation reveals that bimodal distribution was indeed observed in silica and high purity silicon dioxide at higher implantation conditions $[17,16,14]$. The ion energy or the current density is higher in these cases, nearly by an order of magnitude.

For example, Hosono et al, reports a bimodal distribution of Copper implanted into $\mathrm{SiO}_{2}$ glass at a fluence of $6 \times 10^{16}$ atoms.cm ${ }^{-2}$. The ion current density was about $6 \mu \mathrm{A} . \mathrm{cm}^{-2}$ comparable to the conditions used in this setup but the implantation energy was $160 \mathrm{keV}$. Kishimoto et al on the other hand observed bimodal distribution of Copper in silica glass 
at $60 \mathrm{keV}$ implantation energy. But the ion current density was as high as $260 \mu \mathrm{A} . \mathrm{cm}^{-2}$. Ion implantation into $\mathrm{SiO}_{2}$, for example, at $20 \mathrm{keV}$ is not shown to produce a bimodal distribution[24, 97]. Closer examination of the thermal conductivity of these material can explain the need for higher implantation energy or current density for silica or $\mathrm{SiO}_{2}$. Literature suggests the thermal conductivity of hydrogenated DLC to be $0.3 \mathrm{~W} .(\mathrm{mK})^{-1}$ [43]. This is an order of magnitude lower than silica or silicon dioxide which have thermal conductivity in the range of few W. $(\mathrm{mK})^{-1}$. The difference in implantation conditions thus directly correspond to the difference in thermal conductivity. This also explains why similar bimodal distributions are not observed in other materials such as silicon at room temperature implantation.

The model developed so far for implanted DLC closely takes off from the model given by Hosono et al. In their report, they attributed the observed bimodal distribution to diffusion effects arising from thermal spikes. On the other hand, Kishimoto et al, concentrated on the effects of dose rate on the bimodal distribution. They attribute the bimodal distribution to result from the heating effects induced by high current density ion beams and showed direct correlation between the dose rate and the distribution. This was in contrast to study from Hosono et al, where they did not observed any dependency on ion current density / dose rate. Dubiel et al have observed similar effect upon Ag implantation into soda lime glass. They however focused on the stress experienced by the Ag nanoparticles within the dielectric matrix to explain the bimodal distribution. Thus there is no clear model explaining the measured distribution so far.

In this study, the evolution of bimodal distribution of Co in DLC was studied in detail. While there are different perspectives recorded in literature for the cause of bimodal distribution, the results obtained so far in this study tilt the cause of bimodality to be associated with the atomic rearrangements and diffusion effects caused by collision cascades, thermal spikes and defect formation.

\subsection{Conclusion}

In summary, this chapter presents some of the important results on Co distribution and nanoparticle formation in Co implanted DLC. The chapter began with the results from simulation of the ion implantation process using the widely used theoretical model provided by DTRIM. DTRIM provides a general understanding about the distribution of implanted ions in DLC matrix along with details about sputtering, damage distribution and defect formation during implantation. They also provide a base for comparing the experimental results. The experimental details of the actual implantation process was also covered in this 
chapter. Co was implanted into DLC at $30 \mathrm{keV}$ energy with a fluence varying from 0.85 to $20 \times 10^{16}$ atoms.cm ${ }^{-2}$. The implanted films were then investigated using HR-RBS to probe Co distribution and TEM to image the sample cross-section. Both techniques revealed several unique findings that did not conform to theory. The major findings were

- Bimodal distribution of implanted Co

- Chemical interaction of Co with the base matrix resulting in nanoparticle formation

- Presence of larger nanoparticles in the near surface region followed by depletion region further followed by a region consisting of smaller nanoparticles

- Significant changes in the density of the material upon implantation

The results were explained by a model considering the atomic rearrangement, diffusion effects and nanoparticle formation caused by collision cascades, thermal spikes, precipitation and defect formation. In particular one of the important cause for bimodal distribution and a major basis for the validity of the model lies in DLC being an excellent thermal insulator. In the next chapter, this particular basis will be tested by analyzing the ion distribution in Co implanted amorphous carbon films. The thermal conductivity of amorphous carbon is similar to silica and is an order of magnitude higher than that of DLC. Comparing the results of ion implantation in amorphous carbon films with implanted DLC films will test the validity of the proposed model. 



\section{Chapter 6}

\section{Structural effects of hydrogen incorporation}

\subsection{Introduction}

Hydrogen plays an important role on the properties of DLC. The effects of ion implantation in DLC is dependent to a great extent on the presence of hydrogen in DLC films. In the previous chapter, the distribution and configuration of implanted Co atoms in DLC was covered. A model was presented to explain the unique bimodal distribution and nanoparticle arrangement within DLC. In this chapter, the first section presents a comparison of ion distribution in carbon films in presence and absence of hydrogen in the base matrix. The results obtained will be discussed in light of the model proposed in the previous chapter. Once the effect of hydrogen on ion distribution is discussed, the next section presents the effect of ion implantation on hydrogen distribution. Hydrogen being the lightest element gets massively rearranged upon Co implantation. The changes in the hydrogen distribution upon implantation was measured by RNRA and are presented in this section. This is also the first time such a study has been conducted in DLC. The implications of the hydrogen rearrangement is discussed from the overall perspective. This section is finally followed by results from Raman spectroscopy on implanted DLC films. This technique provides details about the effect of ion implantation on the overall structure and hybridization of DLC. In the same section, the Raman spectra of implanted DLC films and a:C films will be compared to highlight the difference in the effects of ion implantation on carbon films in presence and absence of hydrogen. This chapter concludes the study on the structural properties of Co implanted DLC films. 


\subsection{Effect of hydrogen on Co profile}

In the previous chapter, a model was presented to explain the evolution of bimodal Co distribution in DLC. According to the model, one of the primary factor responsible for the bimodal distribution was that DLC is an excellent thermal insulator. Comparison with literature showed that other thermal insulators such as $\mathrm{SiO}_{2}$ did not show bimodal distribution at the implantation conditions used in this study. However, increasing the ion implantation energy to $160 \mathrm{keV}$ resulted in bimodal ion distribution. This was explained by the difference in the thermal conductivity of DLC compared to $\mathrm{SiO}_{2}$. Shamsa et al have shown that DLC was measured to have a thermal conductivity of $0.3 \mathrm{~W} /(\mathrm{mK})$, while $\mathrm{SiO}_{2}$ was measured to have a thermal conductivity of $1.4 \mathrm{~W} /(\mathrm{mK})$ [98]. In other words, according to the proposed model, increasing the thermal conductivity from 0.3 to $1.4 \mathrm{~W} / \mathrm{mK}$ prevents the formation of bimodal distribution. But this is not a direct comparison. $\mathrm{SiO}_{2}$ is vastly different from DLC in many aspects. Hence the comparison may not yield conclusive results.

In order to test the proposed model, and yield conclusive proof about the origin of bimodality in this study, amorphous carbon $(\mathrm{a}: \mathrm{C})$ films were implanted with Co to a fluence of $4 \times 10^{16}$ atoms.cm ${ }^{-2}$. Shamsa et al, in their study have shown that a:C films have thermal conductivity in the order of $3.5 \mathrm{~W} /(\mathrm{mK})$, which is well above the thermal conductivity of $\mathrm{SiO}_{2}$. In the below parts, the Co distribution in a:C films are analyzed and compared to Co distribution in DLC (a:C-H) films.

\subsubsection{DTRIM Simulation}

Similar to the approach in previous chapter, DTRIM simulations were first performed in order to understand the difference in Co distribution with lack of hydrogen, as predicted by theory. Hence, DLC deposited by pure carbon ions are considered for this study. Lack of hydrogen, which constitutes 25 at.\% of the film is expected to result in significant changes in the base matrix that can affect the implantation conditions. As a start, the input file for the DTRIM simulations is now modified to contain only one element $(\mathrm{C})$ in the target. The density of the target was also varied from 1.51 g.cm ${ }^{3}$ to 2.7 g.cm $\mathrm{cm}^{3}$. 


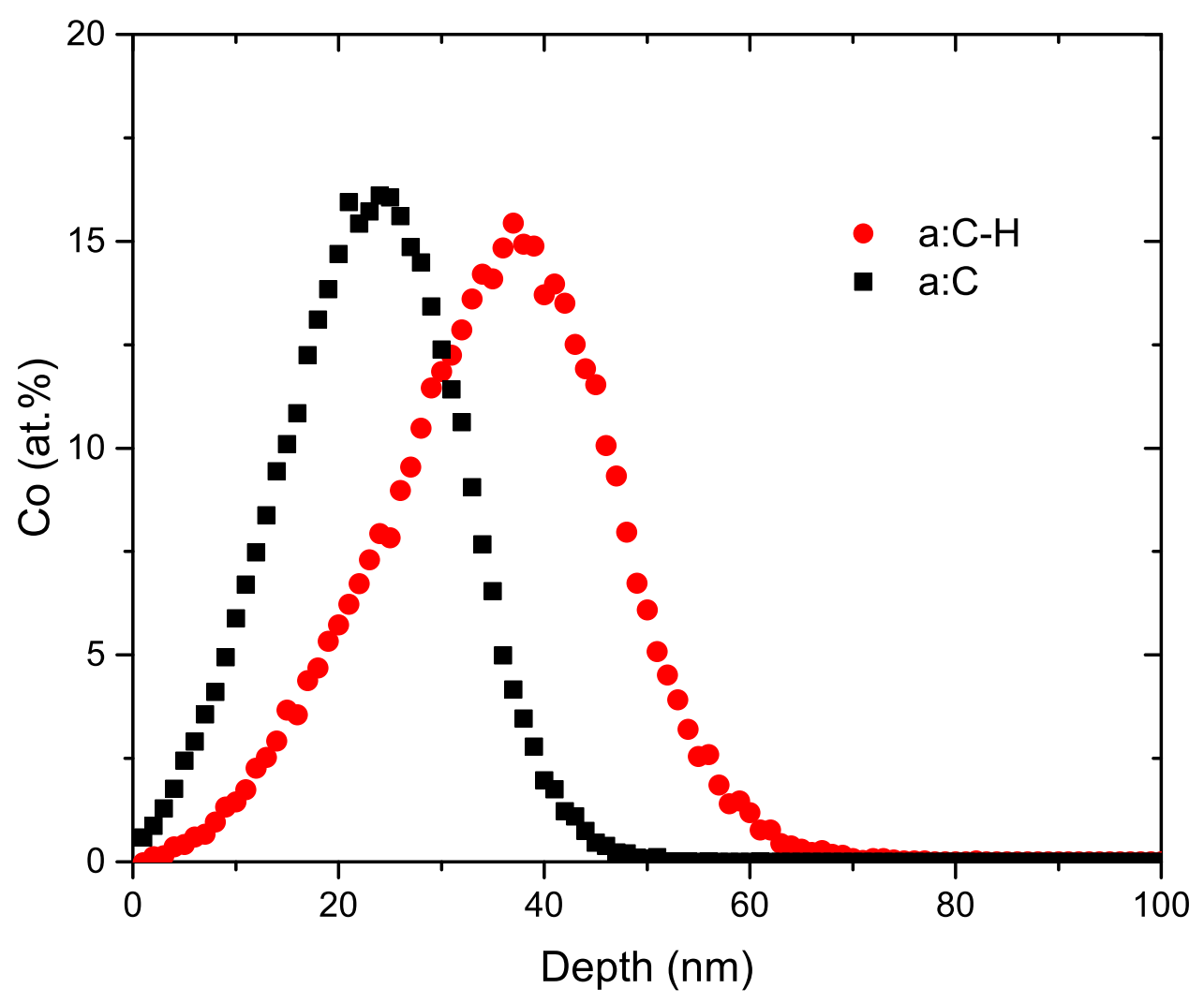

Figure 6.1: Comparison of DTRIM predicted Co implantation profile in a:C and a:C-H films for a fluence of $4 \times 10^{16}$ atoms.cm ${ }^{-2}$. It can be observed that in a:C films the Co peaks about $10 \mathrm{~nm}$ closer to the surface due to the increased stopping.

Figure 6.1 shows the predicted Co distributions for a:C and a:C-H films. The major difference between both the films is the mean projected range which reduces from $34 \mathrm{~nm}$ for a:C-H film to $24 \mathrm{~nm}$ for a:C film. The straggling or the half width of the distribution also reduces from $11 \mathrm{~nm}$ to $8.8 \mathrm{~nm}$. While Co is not expected to reach the surface in the case of a:C-H films, a minor concentration $(0.6$ at.\%) of Co is expected to be present at the surface of the a:C films after Co implantation. The changes in the observed distributions of $\mathrm{Co}$ in figure 6.1 can be attributed to the increased density of DLC films due to absence of hydrogen atoms. The increased stopping resulting from higher density and absence of hydrogen atoms causes the shift in projected range towards surface.

\subsubsection{Cobalt distribution}

Amorphous carbon films implanted with Co to a fluence of $4 \times 10^{16}$ atoms.cm $^{-2}$ (a:C-Co 4) were measured by HR-RBS under the same experimental setup used before. Figure 6.2 
shows the SIMNRA fitting to the measured HR-RBS spectrum.

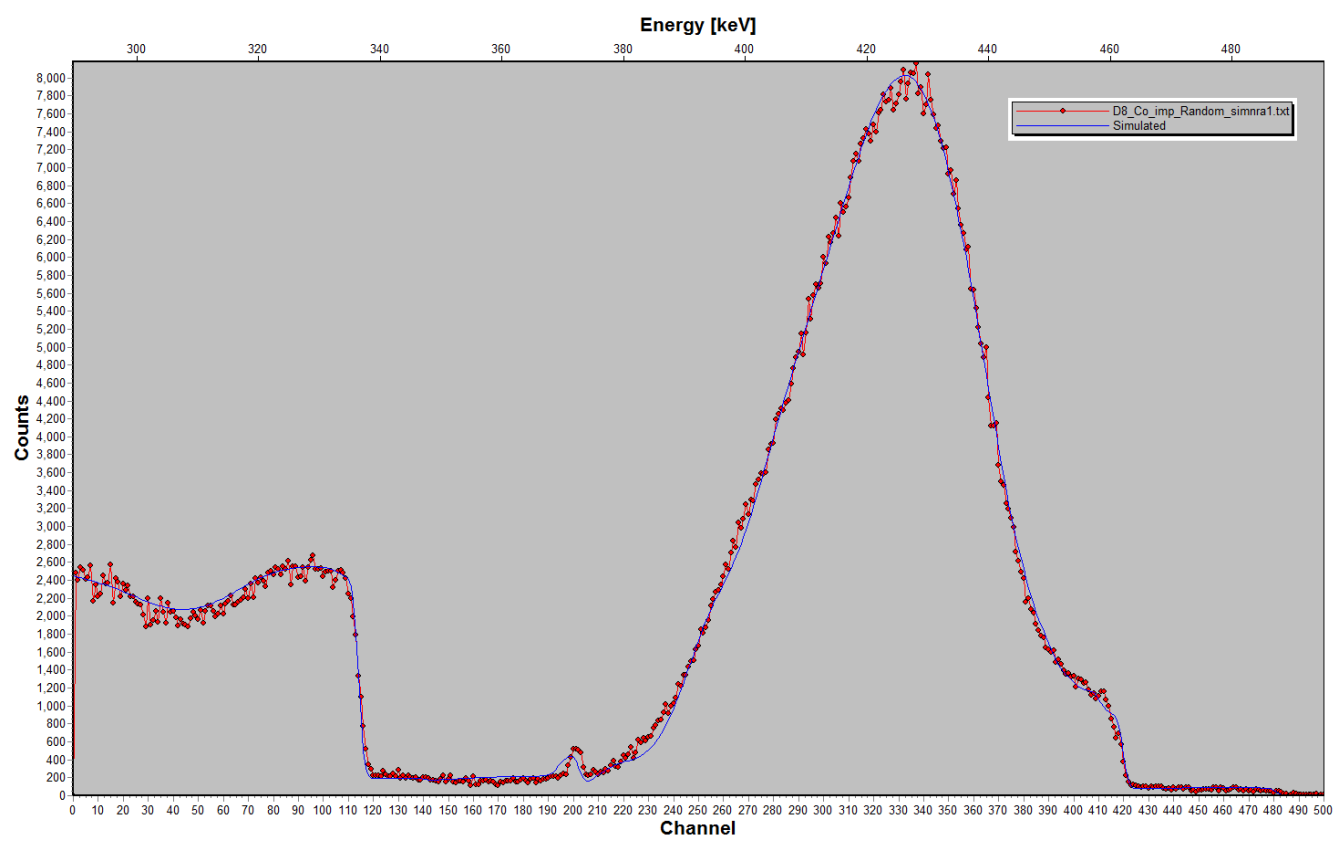

Figure 6.2: SIMNRA fitting of amorphous carbon films implanted with Co to a fluence of $4 \times 10^{16}$ atoms. $\mathrm{cm}^{-2}$. The fit shows a unimodal Co distribution upon implantation.

Even before fitting with SIMNRA software, it can be clearly seen from the raw data that the Co distribution is unimodal in nature. Bimodal distribution is not observed upon Co implantation into a:C films. For in-depth analysis the raw data is fit with SIMNRA target. The target is constructed of 46 layers with a Co dose of $4.2 \times 10^{16}$ atoms.cm ${ }^{-2}$ The target also takes into account the $\mathrm{O}$ and Ta peak observed in the raw data. Oxygen peak can be explained by the surface presence of Co in a:C film. Co in the surface oxidizes to form cobalt oxide. The presence of Ta can be attributed to the sputtering of Ta mask used for holding the sample. 


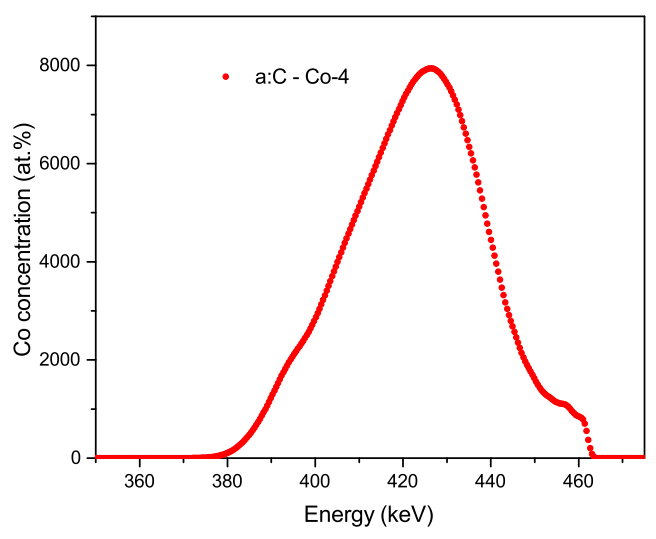

(a) Co profile from HR-RBS spectrum

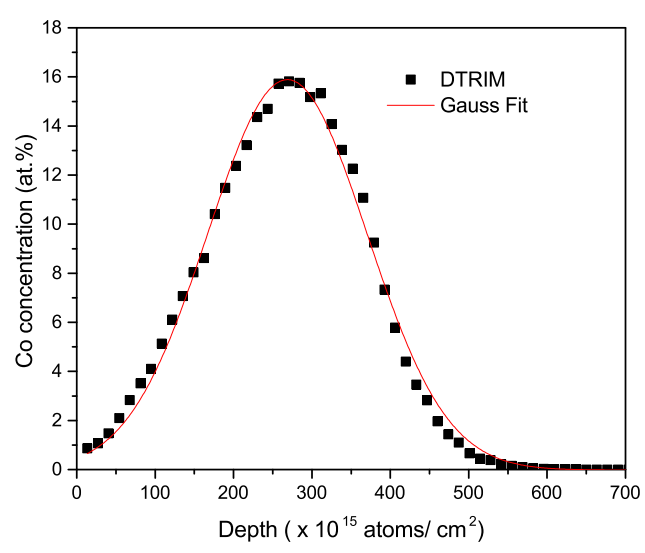

(c) DTRIM prediction of Co depth profile

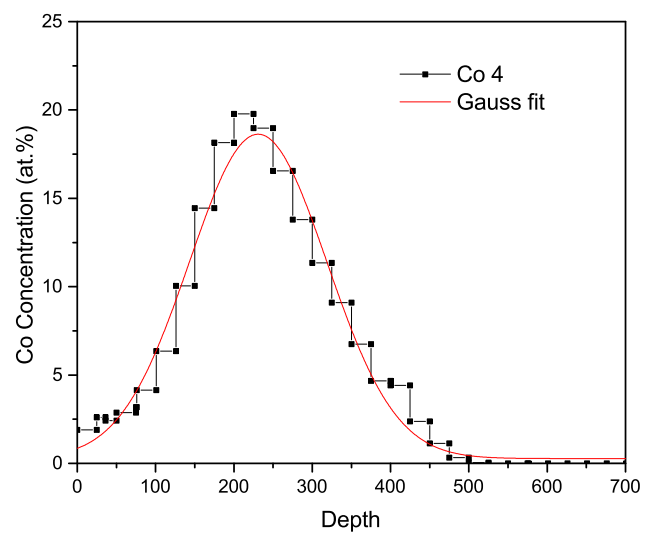

(b) Co depth profile from SIMNRA fitting

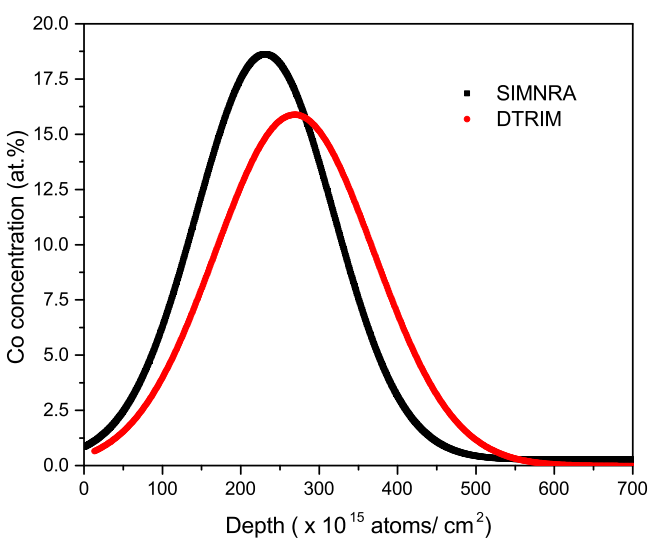

(d) DTRIM Simulation vs Experimental measurement

Figure 6.3: Analysis of HR-RBS analysis of Co distribution in amorphous carbon films implanted with Co to a fluence of $4 \times 10^{16}$ atoms.cm ${ }^{-2}$. The experimental measurement agrees with the DTRIM prediction on the nature of distribution (unimodal) but the Co peak is measured to be closer to the surface and narrower than the predicted distribution.

Figure 6.3 contains the analysis of Co distribution in a:C film. Figure 6.3 (a) shows the Co distribution in counts vs energy plot. The Co peak is observed at $426 \mathrm{keV}$ and is observed to extend upto $380 \mathrm{keV}$. There is a significant concentration of Co present in the surface. This indicates that there is still some diffusion effects caused due to ion implantation, however the diffusion is not significant enough to cause a bimodal distribution. Another important feature that is visible in the spectrum is the asymmetric nature of the distribution. Co distribution is slightly skewed towards the interface. Figure 6.3 (b) shows the Co depth profile extracted from the SIMNRA fit. The peak concentration is measured to be 20 at.\% at $200-225 \times 10^{15}$ atoms.cm ${ }^{-2}$. The surface concentration of Co is measured to be 2 at.\%. The depth profile is fit with a Gauss distribution to obtain a continuous profile of Co 
distribution. Figure 6.3 (c) shows the predicted profile from DTRIM. The peak concentration predicted was predicted to be 15.8 at.\%. The distribution is also fit with Gauss function to obtain a continuous distribution. Both these fits are compared in figure 6.3 (d). It can be seen that even in the absence of bimodal distribution the DTRIM predicted profile do not match with the measured distribution. The Co peak is predicted at $270 \times 10^{15}$ atoms.cm $^{-2}$ which is at least $50 \times 10^{15}$ atoms.cm ${ }^{-2}$ away from the measured peak. The mismatch can be explained when considering the TEM images of the Co implanted a:C films.

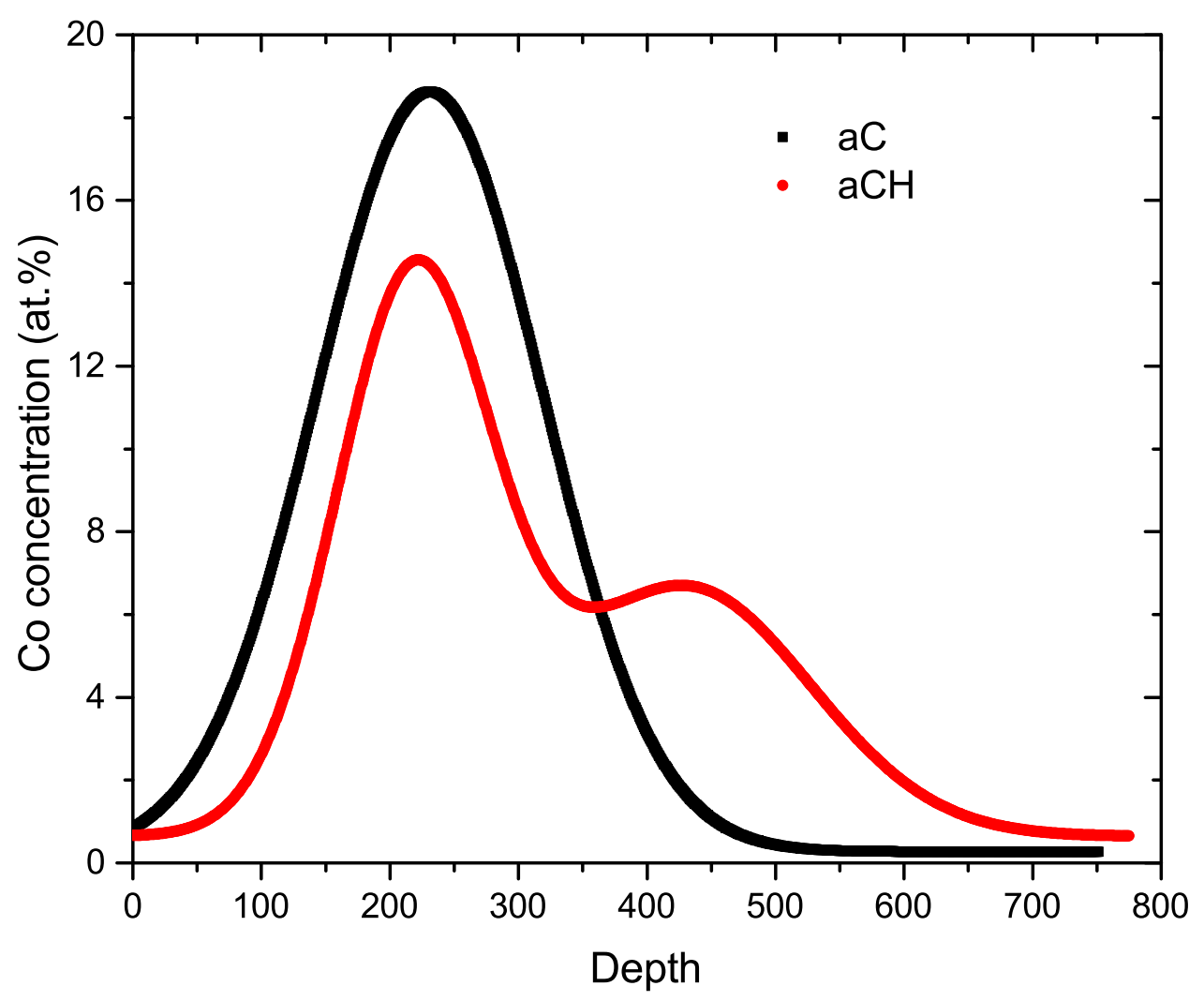

Figure 6.4: Comparison of Co distribution in Co implanted a:C film and a:C-H films (for a fluence $4 \times 10^{16}$ atoms.cm ${ }^{-2}$ ).

Figure 6.4 highlights difference in Co distribution of Co implanted a:C films and Co implanted a:C-H films. The biggest difference lies in the bimodal nature of Co distribution in a:C-H film. This implies that by tuning the hydrogen concentration in DLC films the distribution of Co can be varied from a unimodal distribution to bimodal distribution. However, interestingly the Co peak position in a:C film coincides with the surface peak position for a:C-H film. This also validates our proposed hypothesis that thermal conductivity play a 
major role in deciding on the distribution of implanted ions within the matrix.

\subsubsection{Cross sectional imaging}

The cross-section of Co implanted a:C films were imaged by TEM. The samples were prepared using the classic method [99]. The images recorded are shown in figure 6.5.
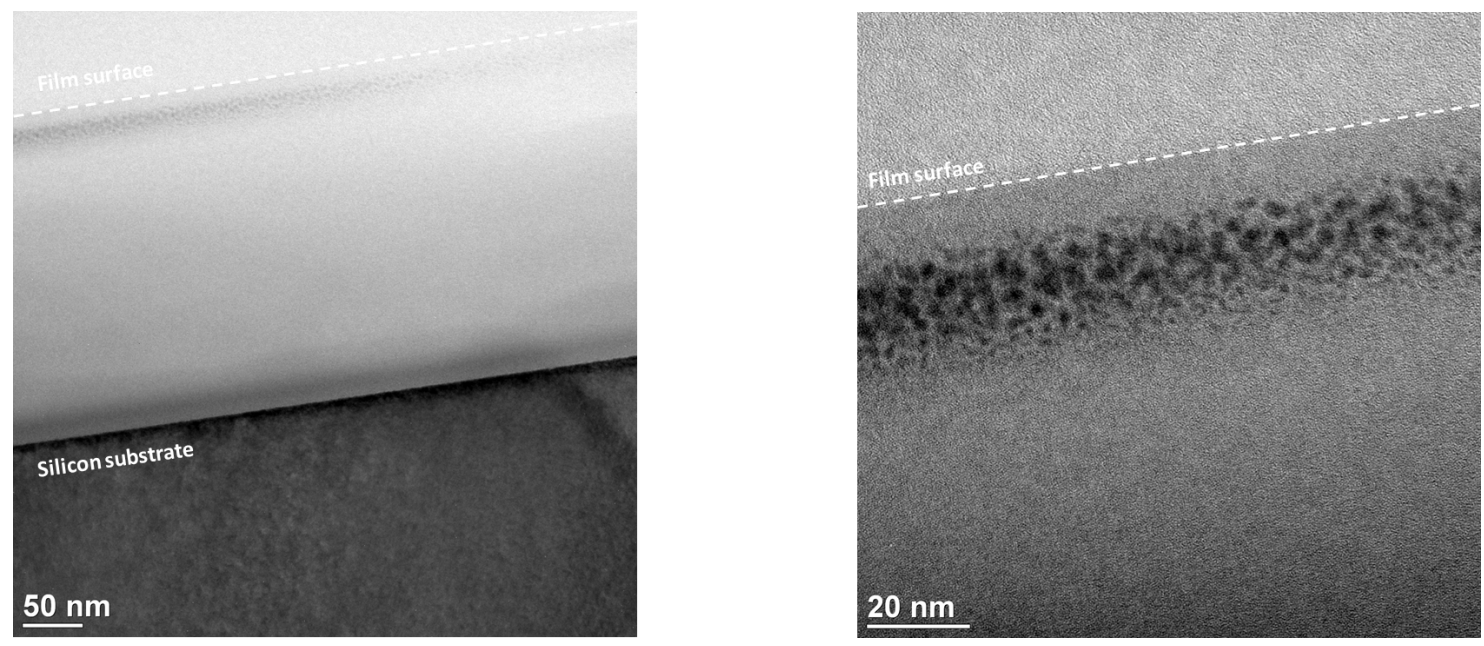

Figure 6.5: Cross sectional TEM images of amorphous carbon films implanted with Co to a fluence of $4 \times 10^{16}$ atoms.cm ${ }^{-2}$ showing nanoparticle formation. The film surface is marked with white dashed lines.

The TEM image shows the formation of nanoparticles containing Co in the a:C film. Similar to a:C-H films, no nanoparticles were observed to be present in the region close to surface even though significant amount of Co is measured to be in the surface region by Rutherford backscattering. This indicates dilution of Co in DLC. The average nanoparticle size is estimated to be about $2 \pm 1.2 \mathrm{~nm}$. The nanoparticle formation is concentrated at the projected range indicating that the diffusion effects do not dominate at this region. This is direct contrast to that observed in a:C-H film. This also gives an opportunity to look at the effects of nanoparticle formation on Co distribution, since in earlier samples presence of diffusion effects compensated the effects of precipitation. Consider figure 6.3 (d), the measured distribution was narrower than that predicted by theory. The projected range was also measured to be closer to surface than predicted. This mismatch is a direct effect of nanoparticle formation. Since Co atoms aggregate to form nanoparticle their distribution becomes narrower and hence the peak concentration rises within the region. The shift in projected range could result from the increased stopping experienced due to precipitation. 

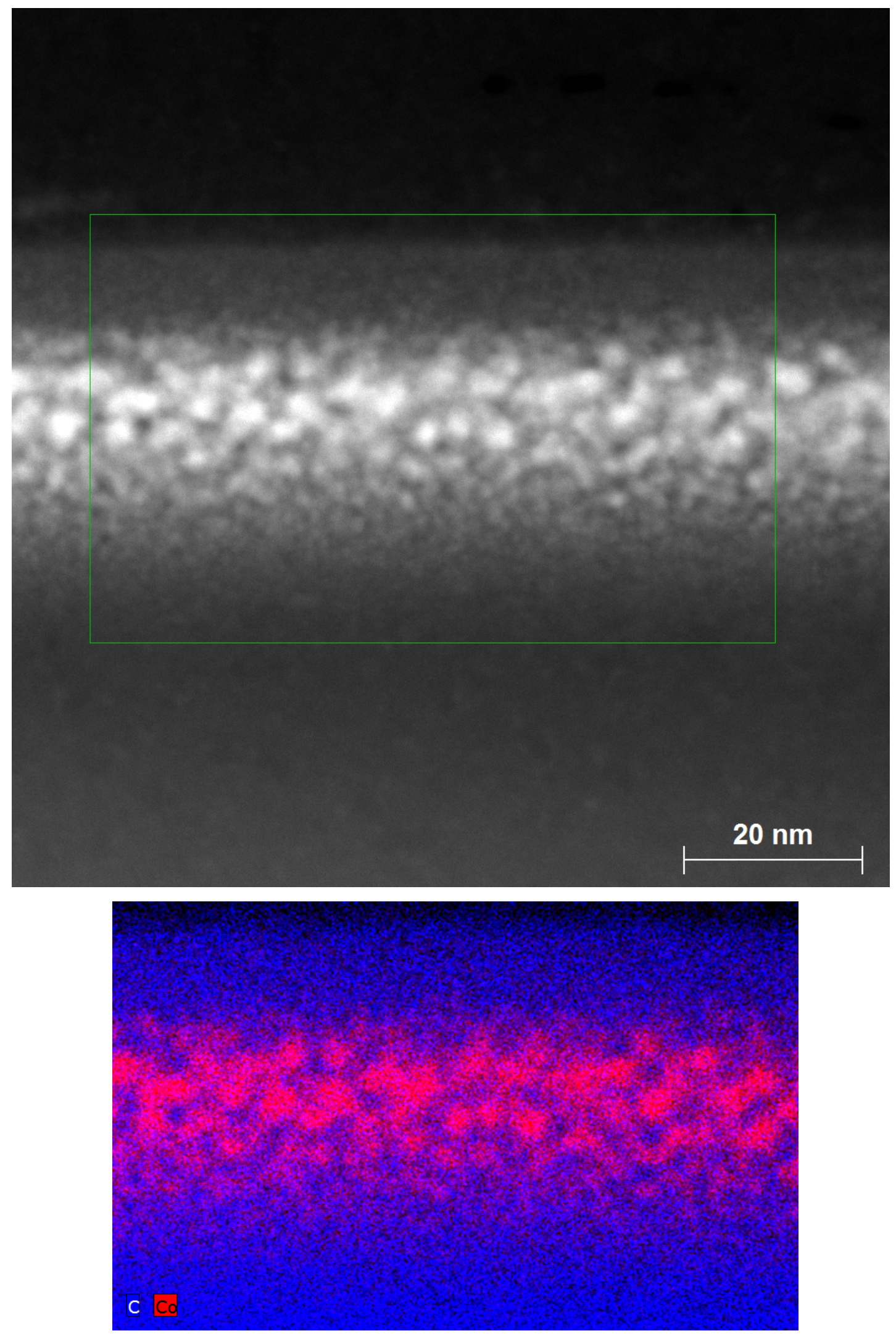

Figure 6.6: Areal composition mapping of amorphous carbon films implanted with Co $(4 \times$ $10^{16}$ atoms.cm ${ }^{-2}$ ), showing the distribution of Co within the carbon film. 
STEM areal mapping was also performed on the sample to identify the different elements making up the matrix. The image shows that the nanoparticles are primarily composed of Co and possibly of C. Since this technique is not sensitive towards minor concentration of the elements, diluted Co in the near surface region or the presence of sputtered Ta is not observed in the image.

This in principle concludes the section on Co distribution in a:C film. The objective of this experiment was to check the validity of the hypothesis which is achieved by this analysis. Now that hydrogen is inferred to play a key role in determining the distribution of Co, it is worthwhile to measure the effect of Co implantation on the hydrogen distribution within the implanted films.

\subsection{Nuclear Reactions - Hydrogen measurement}

Ion implantation in general causes massive redistribution in the elements composing the base matrix. Implanted DLC is mainly constituted of $\mathrm{Co}, \mathrm{H}$ and $\mathrm{C}$. The distribution of Co has already been covered and the difference in Co distribution in presence and absence of hydrogen is also discussed. In this section, the objective is to measure the hydrogen distribution in the implanted films. The technique used to measure the hydrogen profile is Resonant Nuclear Reaction Analysis (RNRA). The method works by directly probing the hydrogen nucleus present within in the sample by nuclear reactions. A brief introduction and the experimental setup used for this analysis is provided in chapter 2 and 4 respectively. This technique has been previously used to measure the hydrogen concentration of as-deposited DLC films in chapter 4 . 


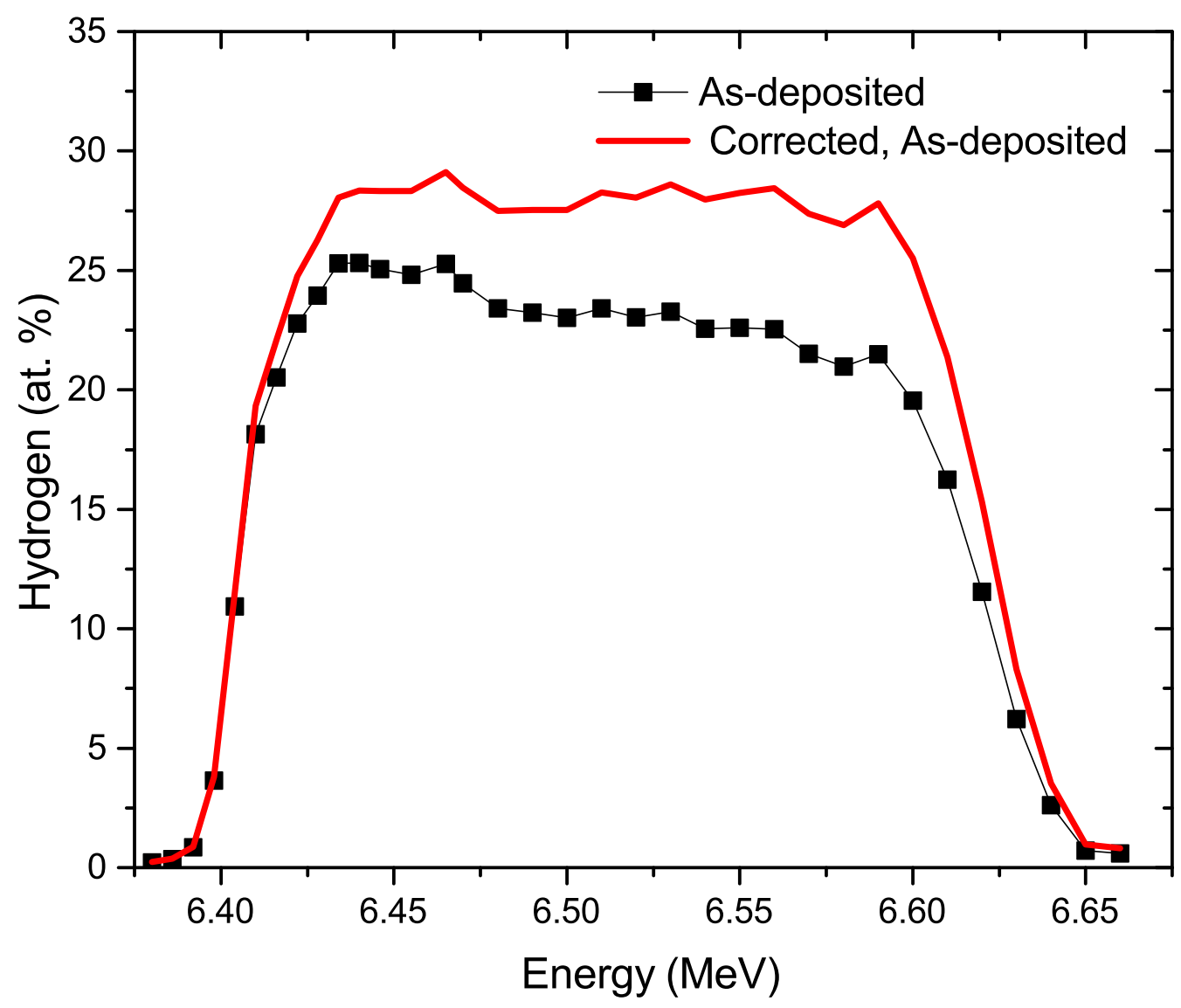

Figure 6.7: Hydrogen profile in as-deposited DLC films synthesized by MSIBD discussed in chapter 4 .

Figure 6.7 shows the hydrogen profile measured by RNRA for as-deposited DLC synthesized by MSIBD. The figure is already analyzed in chapter 4 and is presented here to act as reference for the implanted films. In the figure the plot in red curve represents the hydrogen profile corrected for hydrogen loss due to energetic ion beam exposure during the measurement. For as-deposited DLC this correction can be performed by measuring the loss of hydrogen at a particular point with respect to charge accumulation. From the data, rate of hydrogen loss was calculated in at.\%. $\mu \mathrm{C}^{-1}$ and the corresponding correction can be performed at the different points of the plot. For an accurate description the hydrogen loss rate at a particular point was taken to be dependent on the original hydrogen concentration present in the sample. One assumption that was used in this correction process is that the hydrogen is bonded in the same manner at all depths of the film. This assumption 
is important because the loss of hydrogen was measured at one particular energy $(6.5 \mathrm{MeV})$ and applied throughout the film. This energy corresponds to a DLC region of particular depth. The hydrogen loss rate measured for this particular depth is assumed to represent the whole sample. This is a good approximation since there is no particular reason or basis for hydrogen to be bonded in different manners at different depths of the film. Ion beam deposition is a uniform process with time and fluence and hence should not result in significant variations within different depths of the film. However, this assumption can not be safely applied to implanted DLC films. Ion implantation affects a particular region of the film. The damage and ion distribution is not uniform and hence depending on depth the effect of ion implantation in hydrogen can be different which in turn affects the bonding and configuration of hydrogen atoms within the film. This implies that the hydrogen loss correction applied to the as-deposited film can not be applied to implanted films. For the implanted films, analysis and comparisons will be limited to the raw data only.

In this section the discussion will be concentrated towards the hydrogen profile without concerning about the hydrogen loss due to effusion. The first step is to summarize the list of samples that will be presented in this section.

Table 6.1: Samples measured by RNRA

\begin{tabular}{|c|c|c|c|c|c|}
\hline S.No & DLC & Synthesis & Ion & Fluence $\left(\times 10^{16}\right.$ atoms.cm & \\
& & Sample Code \\
\hline 1 & a:C-H & MSIBD & - & As-deposited & DLC_M1 \\
\hline 3 & a:C-H & MSIBD & Co & 0.8 & Co 0.8 \\
\hline 4 & a:C-H & MSIBD & Co & 1.2 & Co 1.2 \\
\hline 5 & a:C-H & MSIBD & Co & 2.4 & Co 2.4 \\
\hline 6 & a:C-H & MSIBD & Co & 7 & Co 7 \\
\hline 7 & a:C-H & MSIBD & Co & 12 & Co 12 \\
\hline 8 & a:C-H & DIBD & Co & 4 & Co $4 \_D$ \\
\hline
\end{tabular}

Table 6.1 lists the sample details and their respective codes. Sample Co 4 _ D alone is synthesized by Co implantation into DIBD synthesized DLC film. DIBD films contain slightly higher hydrogen content and hence are chosen for evaluation. The implanted samples cover a range of fluence starting from $0.8-12 \times 10^{16}$ atoms. $\mathrm{cm}^{-2}$. The analysis will begin with sample Co 0.8 and Co 1.2, where the Co distribution is still unimodal followed by analysis on sample Co 2.4 and Co 7 where the Co distribution is measured to be bimodal. This will be followed by results from DIBD synthesized DLC implanted with Co and Co 12 sample where it is expected to have reached a steady-state condition upon implantation.

Hydrogen profile measured from sample Co 0.8 and Co 1.2 are presented in figure 6.8. 


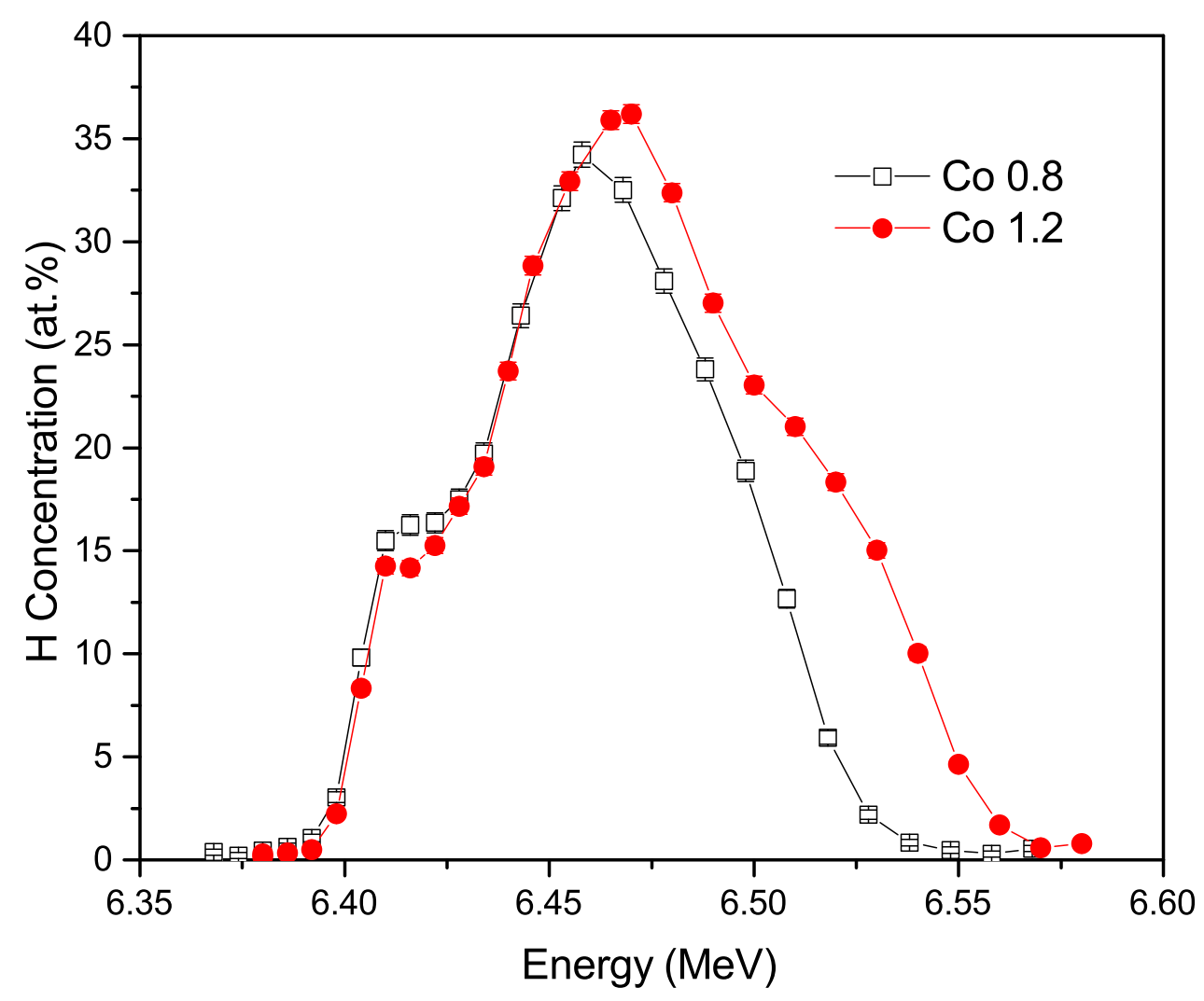

Figure 6.8: RNRA profile of Co 0.8 and Co 1.2 showing hydrogen redistribution after Co implantation. Hydrogen depletion is observed in the near-surface region followed by hydrogen accumulation at deeper regions.

The hydrogen profile is presented in concentration vs energy plot. The energy axis in these plots directly correspond to the input energy of the ${ }^{15} \mathrm{~N}$ beam used for the measurement. As explained in the previous chapters, resonant nuclear reaction occurs at a specific energy. For this particular nuclear reaction, the resonant energy is $6.385 \mathrm{MeV}$. As the beam energy is increased, the ions lose energy due to electronic stopping which shifts the resonance to a particular depth within the films. Thus the entire film thickness can be covered by sweeping the energy in steps. Hence the energy scale directly corresponds to the depth scale. Since implanted DLC is shown to have significant variations in density within the sample it is not accurate to represent the plot in depth scale. Thus it is safe to proceed with the energy scale with the indication that increase in energy corresponds to an increase in depth.

Figure 6.8 shows the hydrogen profile measured for sample Co 0.8 and Co 1.2. Hydrogen profile of Co 0.8 is represented by open black boxes and Co 1.2 by closed red circles. The 
first observation is that the hydrogen distribution is not uniform within the sample. For as-deposited films, as seen in figure 6.7, the hydrogen profile is approximately a plateau with a concentration of $\sim 28$ at. \% . However, implanted DLC films show a hydrogen peak in the measured distribution.

The background noise of the spectrum is measured to be $<0.4$ at.\%. First significant rise in the signal above the background noise is observed at $6.386 \mathrm{MeV}$. This energy is just 1 $\mathrm{keV}$ above the resonance energy, however beam broadening effects does not allow us to fully probe the surface concentration. From that point there is a rapid rise in the signal upto 6.41 MeV. This region is generally referred to as surface edge and the concentration at 6.41 $\mathrm{MeV}$ is taken to reflect the surface concentration. The surface edge of hydrogen in both the samples are vastly similar however the surface concentration are different. For as-deposited films the surface concentration was measured to be 18.1 at.\%. With Co implantation upto $0.8 \times 10^{16}$ atoms.cm ${ }^{-2}$ this surface concentration drops to 15.5 at. $\%$ and implanting the sample further by $0.4 \times 10^{16}$ atoms.cm ${ }^{-2}$ reduces the surface hydrogen concentration to 14.3 at. $\%$.

The next significant feature is the observation of a peak concentration of hydrogen in the spectrum. In Co 0.8, the hydrogen peak is measured to be 34.2 at.\% at $6.458 \mathrm{MeV}$ and for Co 1.2 the hydrogen concentration is measured to be 36.2 at.\% at $6.47 \mathrm{MeV}$. The peak concentration and position is observed to increase with the implantation fluence. From the peak position, the hydrogen concentration drops gradually. In the hydrogen profile for sample Co 0.8 , the drop is continuous upto $6.55 \mathrm{MeV}$ with the measured hydrogen concentration falling within the background noise. In Co 1.2 sample, the drop shifts trend at around 6.5 $\mathrm{MeV}$ and the hydrogen concentration is observed to reach the background level at $6.57 \mathrm{MeV}$. The different energies at which the hydrogen concentration drops to the level of background noise can be taken to directly correspond to the thickness of the sample. There are several features identified from the spectrum and the explanation for the observed features are provided at the end of this section. 


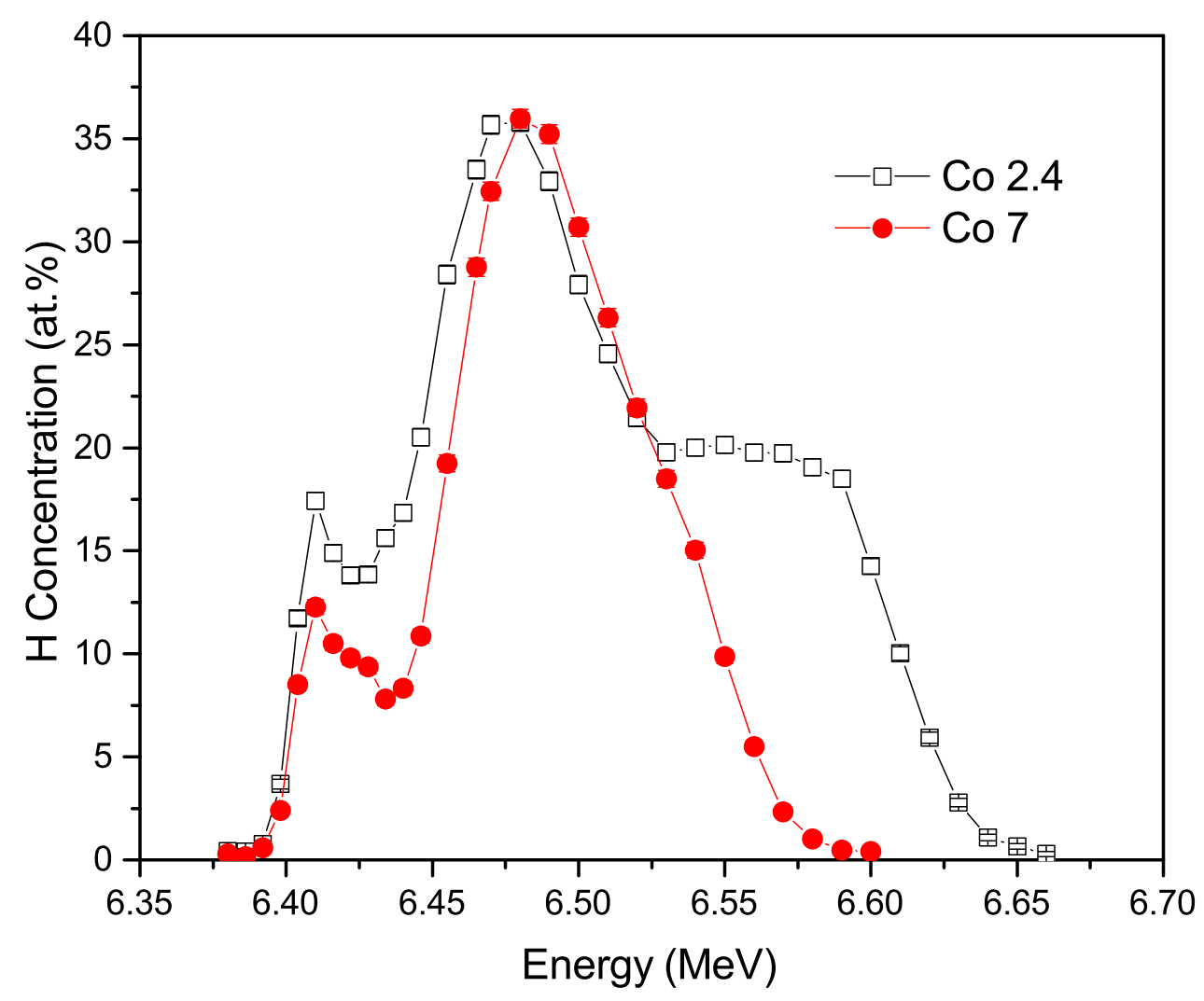

Figure 6.9: RNRA profile of Co 2.4 and Co 7 showing hydrogen redistribution after Co implantation. Hydrogen depletion is observed to have enhanced in the near-surface region followed by similar hydrogen accumulation at deeper regions.

The next samples of interest are Co 2.4 and Co 7 . The hydrogen profiles have not vastly changed from the previous samples. One key difference in these profiles are the observation of hydrogen depleted layers immediately following the surface edge. The surface edge is observed to end at the same $6.41 \mathrm{MeV}$ energy. The surface concentration for Co 2.4 was measured to be 17.4 at.\% while for Co 7 was measured to be 12.3 at.\%. It is interesting to note that Co 2.4 measures greater hydrogen content at the surface than all other implanted sample. However, it is still less than the as-deposited DLC films. Starting from the surface, the hydrogen concentration decreases. The hydrogen minima is measured to be 13.8 at. $\%$ for Co 2.4 and 7.8 at.\% for Co 7. This is the most significant difference observed in Co 2.4 and Co 7 when compared to Co 1.2 and Co 0.8 . This difference can be directly attributed towards the Co distribution. As the implantation fluence is raised from 1.2 to $2.4 \times 10^{16}$ atoms.cm $^{-2}$, the Co distribution evolves from a unimodal distribution to bimodal distribution. There is 
new surface Co peak measured by HR-RBS for samples implanted with higher implantation fluences. This surface peak can be taken to directly account for the depleted hydrogen concentration in the near surface region.

After the hydrogen minima, there is a rapid increase in the hydrogen concentration upto 35.7 at.\% and 35.8 at.\% for Co 2.4 and Co 7. The energy at which this peak is observed is measured to be 6.47 and $6.48 \mathrm{MeV}$. The peak position seems to increase with the implantation fluence. However, the peak concentration saturates around 36 at.\%. In the case of Co 7 the hydrogen concentration drops consistently with increase in beam energy beyond the hydrogen maxima. Hydrogen is measured to drop to noise levels at $6.59 \mathrm{MeV}$. There is a minor shift in the trend of hydrogen decline at about $6.53 \mathrm{MeV}$.

In sample Co 2.4, the decline in hydrogen concentration stops at the same $6.53 \mathrm{MeV}$ where a change in trend was observed in Co 7 sample. At energies higher than $6.53 \mathrm{MeV}$, the hydrogen profile was found to saturate to $\sim 20$ at.\%. The hydrogen concentration again starts declining as the beam energy approaches $6.59 \mathrm{MeV}$ and at $6.65 \mathrm{MeV}$ the measured concentration drops to noise levels. This difference can again be explained by the difference in film thickness. Co 2.4 as observed from TEM images in previous chapter is about 50-60 $\mathrm{nm}$ thicker than Co 7. This increase in thickness results in the plateau region (6.53 to 6.59 $\mathrm{MeV}$ ) observed for Co 2.4 sample.

At this point it is also beneficiary to look at the Co 4 sample where the DLC is synthesized by DIBD. While there will be few minor differences in the hydrogen plots due to use of different base sample, there is no reason to expect any drastic changes in the overall observed trend. 


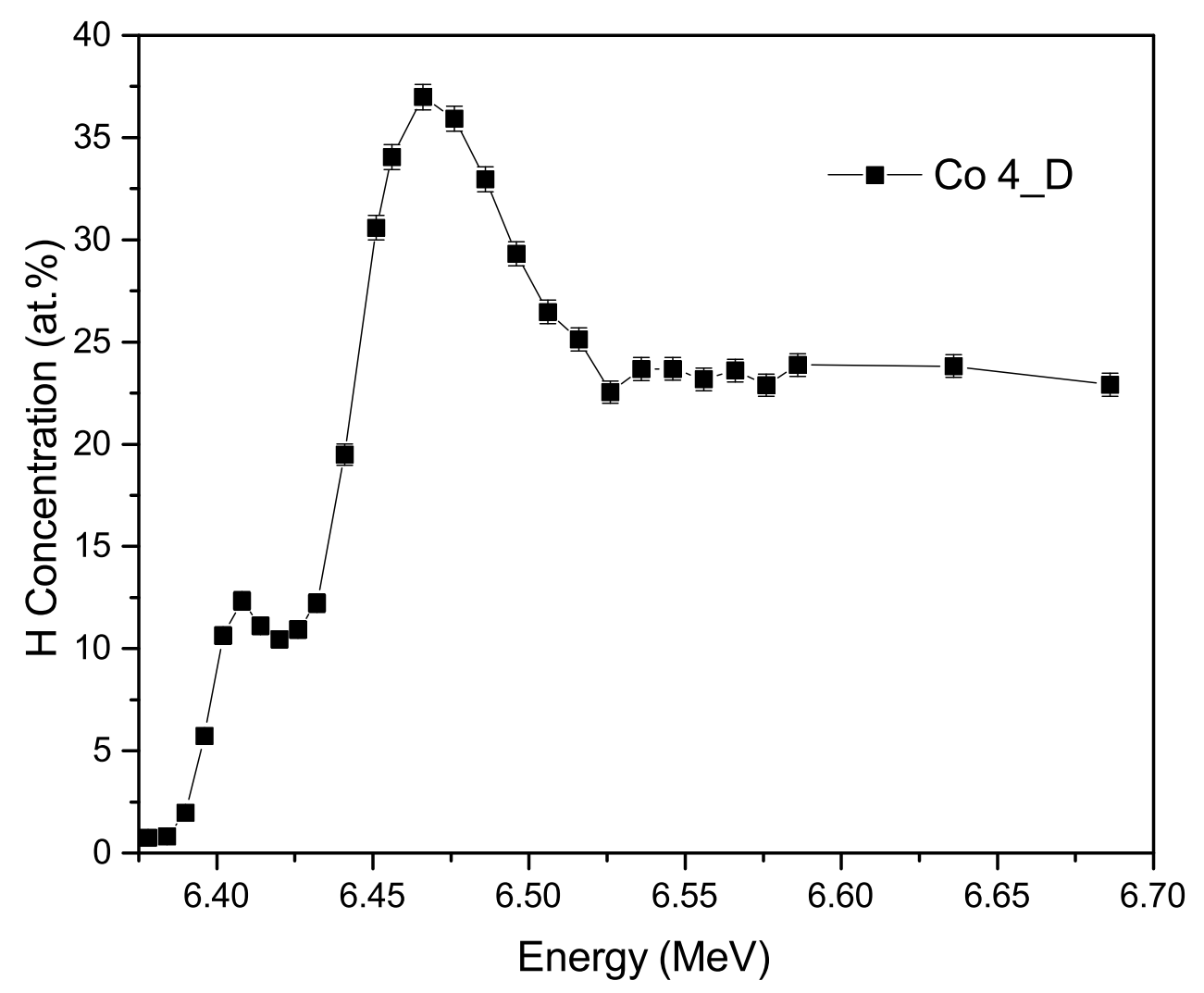

Figure 6.10: RNRA profile of hydrogen distribution in DLC film deposited by direct ion beam deposition and implanted with Co to a fluence of $4 \times 10^{16}$ atoms.cm $^{-2}$. It can be observed DIBD deposited DLC films show similar hydrogen redistribution as observed in MSIBD films.

The profile as expected is similar to the previous sample. There is a surface edge followed by a hydrogen depleted region corresponding to the surface Co peak. This is followed by a hydrogen peak which ends at $6.53 \mathrm{MeV}$ and is followed by saturated hydrogen content of $\sim 24$ at.\%. Since DIBD synthesized films are thicker, the decline of hydrogen concentration to noise levels are not observed within the energy window of measurement. The surface hydrogen concentration was measured to be 12.3 at.\% . In the depleted region the hydrogen minimum was measured to be 10.5 at.\% at $6.422 \mathrm{MeV}$ and the hydrogen peak concentration was measured to be 37 at.\% at $6.468 \mathrm{MeV}$. 


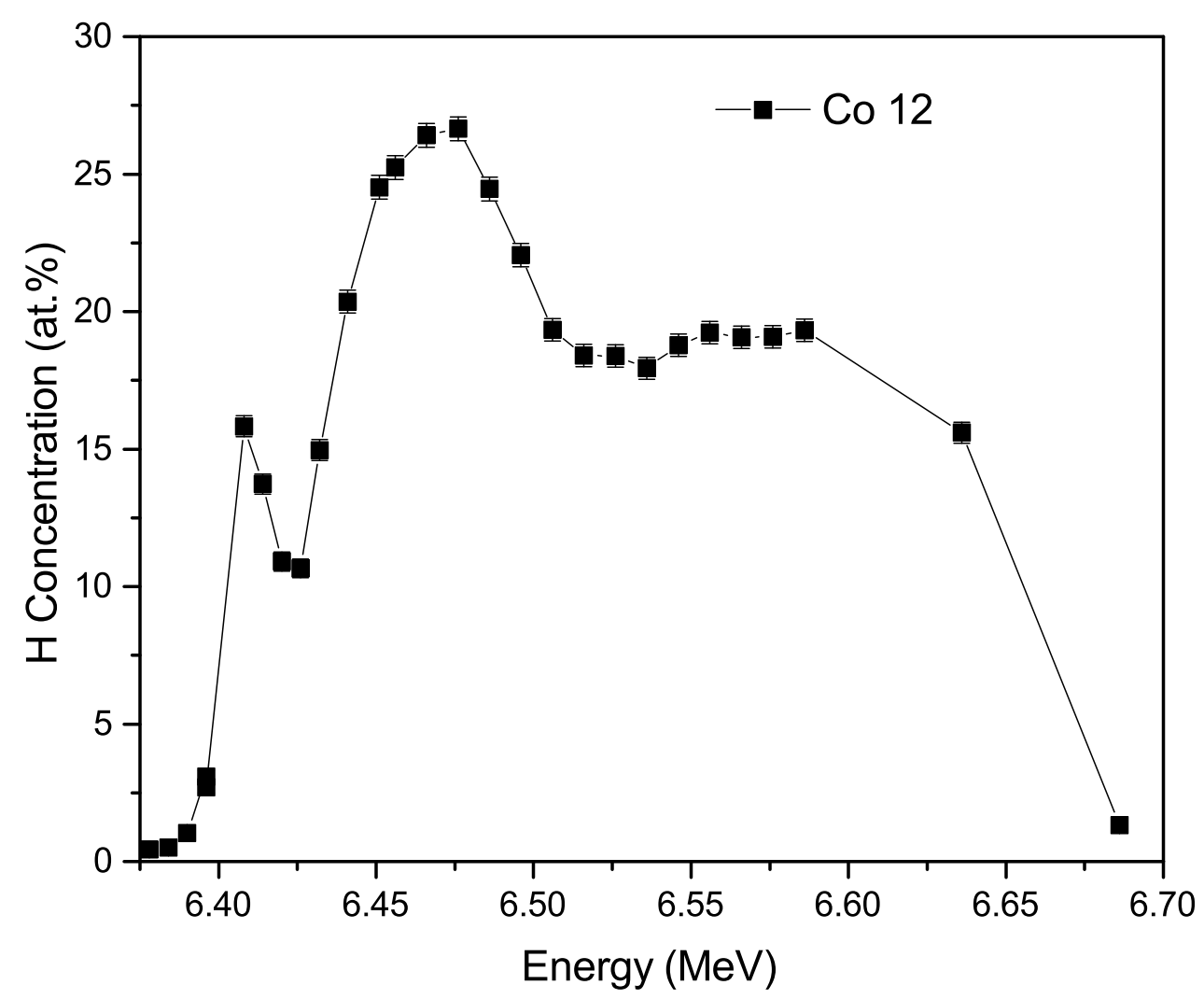

Figure 6.11: RNRA profile of hydrogen redistribution in Co 12 sample. The steady-state condition earlier measured for the Co 12 sample is not observed to affect the hydrogen redistribution caused due to Co implantation.

The final sample that is presented before getting into the analysis of the observed features is Co 12. Co 12 was measured to have attained a steady-state condition with respect to Co distribution by HR-RBS measurements. More details about it can be found in section 5.4.5. The hydrogen distribution for the same sample is shown in figure 6.11. Unlike the Co distribution, the hydrogen distribution do not show any major changes upon reaching the steady-state condition. The distribution has the same features as measured for the previous samples, however minor variations are observed which can best be described quantitatively.

For example, the surface hydrogen concentration was measured to be 15.8 at.\% which is higher than most implanted films. The only implanted film that shows a greater surface hydrogen concentration is Co 2.4. One unifying factor for both the samples are that they are much thicker than the other samples. It was observed so far that there exists a hydrogen depleted region corresponding to Co surface peak. As the fluence increases, the Co 
concentration at the surface peak increases and this leads to greater hydrogen depletion in the near-surface region. However, in this case the depleted hydrogen content is measured to be higher than that measured for Co 7 sample. This could possibly be connected to the observation of a depleted Co region within the surface Co peak for Co 12 sample. Perhaps the most important difference is the peak hydrogen concentration measured in the $\mathrm{H}$ profile. For Co 12 sample the peak hydrogen concentration was measured to be 26.6 at.\% at 6.478 $\mathrm{MeV}$. While the peak position might follow the trend observed so far, the peak concentration is significantly lower than that observed. It is lower by approximately 10 at.\%.

These findings are summarized in table 6.2.

Table 6.2: RNRA profile of Co implanted DLC

\begin{tabular}{|c|c|c|}
\hline Co Fluence & Surface concentration & Charge \\
\hline$\times 10^{16}$ atoms.cm $^{-2}$ & at. $\%$ & $\mu \mathrm{C}$ \\
\hline \hline 0.8 & 15.5 & 3.227 \\
\hline 1.2 & 14.3 & 2.675 \\
\hline 2.4 & 17.4 & 3.425 \\
\hline 4 & 12.3 & 3.632 \\
\hline 7 & 12.3 & 2.82 \\
\hline 12 & 15.8 & 4.227 \\
\hline
\end{tabular}

\begin{tabular}{|c|c|c|c|c|}
\hline Co Fluence & Peak position & Peak concentration & Min position & Min conc. \\
\hline$\times 10^{16}$ atoms.cm ${ }^{-2}$ & $\mathrm{MeV}$ & at.\% & $\mathrm{MeV}$ & at.\% \\
\hline 0.8 & 6.458 & 34.2 & 6.41 & 15.5 \\
\hline 1.2 & 6.47 & 36.2 & 6.416 & 14.2 \\
\hline 2.4 & 6.48 & 35.8 & 6.422 & 13.8 \\
\hline 4 & 6.468 & 37.0 & 6.422 & 10.5 \\
\hline 7 & 6.48 & 36.0 & 6.434 & 7.8 \\
\hline 12 & 6.478 & 26.7 & 6.428 & 10.7 \\
\hline
\end{tabular}

All these data, depicts the following trends:

1. With increasing implantation fluence a near-surface hydrogen depletion region is observed. This region corresponds to the position of surface Co peak. As fluence increases the depletion increases.

2. Ion implantation leads to formation of a hydrogen peak at a certain depth within the implanted film. The peak hydrogen concentration can attain a maximum of 37 at.\%

3. The peak position of hydrogen concentration consistently increases with implantation fluence. These need not necessarily indicate that the peak is attained at a higher depth, 
rather it can point towards the increase in stopping experienced by the ion beam as Co concentration increases.

4. The interface edge of DLC with silicon is represented by decline of hydrogen concentration back to noise levels. This happens at increasingly higher energies for films with greater thickness or greater stopping.

DTRIM simulations, explained in section 5.2, predicted the Co implantation to sputter C more than $\mathrm{H}$ leading to increase in surface concentration of hydrogen. However, RNRA measurements show on the contrary that hydrogen concentration at surface reduces with implantation. There are few possible causes for the said effect: recoils due to implanted ions, effusion as hydrogen molecules or by diffusion of mobile hydrogen atoms. DTRIM is based on ballistics and can thus be expected to predict recoils. Since such a peak is not expected in the simulation the loss can be attributed to the latter effects of effusion and diffusion. This can be explained in detail considering the following figure. 


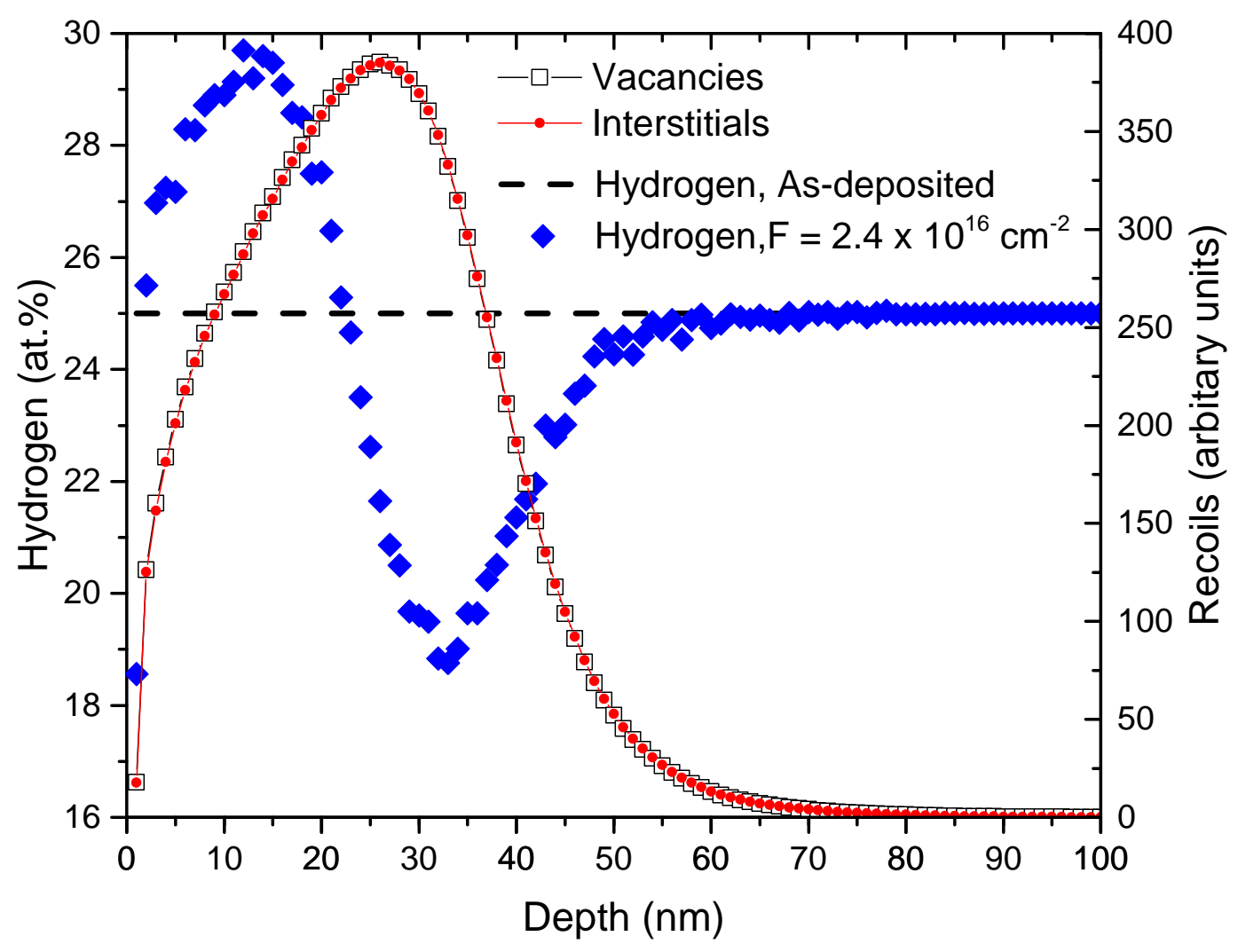

Figure 6.12: DTRIM prediction of hydrogen and vacancy distribution in Co implanted DLC films (Co 2.4 sample).

Figure 6.12 shows the hydrogen profile and vacancy distribution predicted from DTRIM. Experimental measurements presented so far do not agree with the simulations. In order to explain the measured hydrogen profiles, other effects arising from the Co implantation have to be discussed. For this purpose the implications of the model presented in the previous chapter have to be considered.

First, it could be possible that the hydrogen peak at $\sim 6.5 \mathrm{MeV}$ arises from accumulation of hydrogen atoms effusing from the deeper regions of the sample due to ion beam exposure. This accumulation may occur due to the increased density of Co in the implanted DLC film. The densified layer may trap the effusing hydrogen and can lead to the hydrogen peak observed near $R_{p}$. In this case, thicker samples should show a greater accumulation as more hydrogen is present. The hydrogen peak intensity should also increase with charge collected (number of ions bombarded) as higher charge indicates greater beam exposure, which would 
imply growth of the accumulation peak. However, neither of these effects are observed ruling out the possibility of the depth profile to be an experimental artifact.

Second, the observed changes in hydrogen concentration could be purely due to proportional change in elemental concentration arising from introduction of Co atoms. Initial stoichiometry of carbon: hydrogen is measured to be $3: 1$. Addition of Co would imply overall reduction in concentration of both $\mathrm{C}$ and $\mathrm{H}$ even when the number of $\mathrm{C}$ and $\mathrm{H}$ atoms remains the same. However, this does not explain the rise in hydrogen concentration beyond the base (as-deposited) hydrogen concentration $>R_{p}$.

In order to explain this effect we consider the elements of the model proposed to explain bimodal distributions of Co in hydrogenated DLC. Any ion implantation process generates collision cascade that give rise to thermal spikes lasting for few ps [1]. However, only few materials exhibit diffusion effects due to collision cascades $[14,17,16]$. A common feature among these materials is that they are all thermally insulating matrices. In a thermal insulator, the heat generated by the ion beam takes longer time to dissipate. The dissipation time depends on the diffusivity of heat in the material, which is given by thermal conductivity divided by density and specific heat capacity. For example, hydrogenated diamond-like carbon has a heat capacity of $0.825 \mathrm{~J}(\mathrm{gK})^{-1}$ and thermal conductivity of $0.3 \mathrm{mK} . \mathrm{W}^{-1}$. This gives rise to a thermal diffusivity of $0.241 \mathrm{~mm}^{2} \mathrm{~s}^{-1}$, which is even lower than that recorded for silica which is measured to be $0.79 \mathrm{~mm}^{2} \mathrm{~s}^{-1}$ and silica is reported to show bimodal distribution of implanted atoms.

The Co bimodal distribution of the films (refer to section 5.5) evolves due to two factors: (1) diffusion of the implanted atoms from their projected range driven by concentration gradient and defects and (2) formation of nanoparticles during ion implantation. Detailed explanation for their formation can be found in previous chapter. The energy deposited by the incoming ions leads to defects, which in case of hydrogenated DLC represents the dissociation of $\mathrm{C}-\mathrm{H}$ bonds and $\mathrm{C}-\mathrm{C}$ bonds and rehybridization of $\mathrm{sp}^{3}$ to $\mathrm{sp}^{2}$ bonding. Hydrogen being the lightest element in this material has high mobility in its unbound state. Figure 6.12 shows the distribution of vacancies and interstitials. The hydrogen dissociated from carbon can be assumed to have a distribution similar to that of the interstitials with maximum at $26 \mathrm{~nm}$ leading to a concentration gradient of the dissociated hydrogen atoms. Hydrogen in other regions of the films are bonded to carbon atoms and hence do not participate in the diffusion. Based on Fick's law of diffusion, similar to Co, H diffuses from its maximum at 26 $\mathrm{nm}$ towards both the surface and the interface. The energy needed for diffusion is provided by the thermal energy deposited during the collision cascade. The region of this energy deposition is limited by the range of the incoming ions. Once, the diffusing hydrogen atoms move beyond $\mathrm{R}_{p}$, they lack the energy needed to sustain their diffusion leading to hydrogen 
accumulation. It can be observed from the results so far that hydrogen saturates at $\sim 37$ at.\% in the samples.

Even after saturation of hydrogen at the peak position, the depletion region continues to expand with increasing fluence. This can be explained by the effusion of hydrogen at the surface. At the surface diffusing hydrogen atoms can combine to form $\mathrm{H}_{2}$ molecules which is effused out of the sample. Thus the sample surface acts as an infinite sink. On the other hand, diffusion towards the interface stops due to lack of energy due to collision cascades beyond $R_{p}$. This effectively reduces the concentration gradient and stops any net diffusion towards the interface. The stage at which the concentration gradient is neutralized, as obtained from RNRA measurements, is when hydrogen accumulates to 37 at.\%.

In the case of Co 12 sample, the peak concentration is considerably lower and the hydrogen minima concentration is slightly higher than Co 7 sample. This can be attributed to the shift in the depletion layer towards the surface. Thus in this case the vacancy profile shifts its peak further towards the surface thereby lowering the peak hydrogen concentration observed in RNRA.

\subsection{Raman Spectroscopy}

Raman spectroscopy has been used previously in chapter 4 to determine the diamond-like property of the as-deposited films. The effect of deposition energy on the structure of DLC films were examined in detail. In this section, the same technique is used to evaluate the damage induced to DLC by Co implantation. 


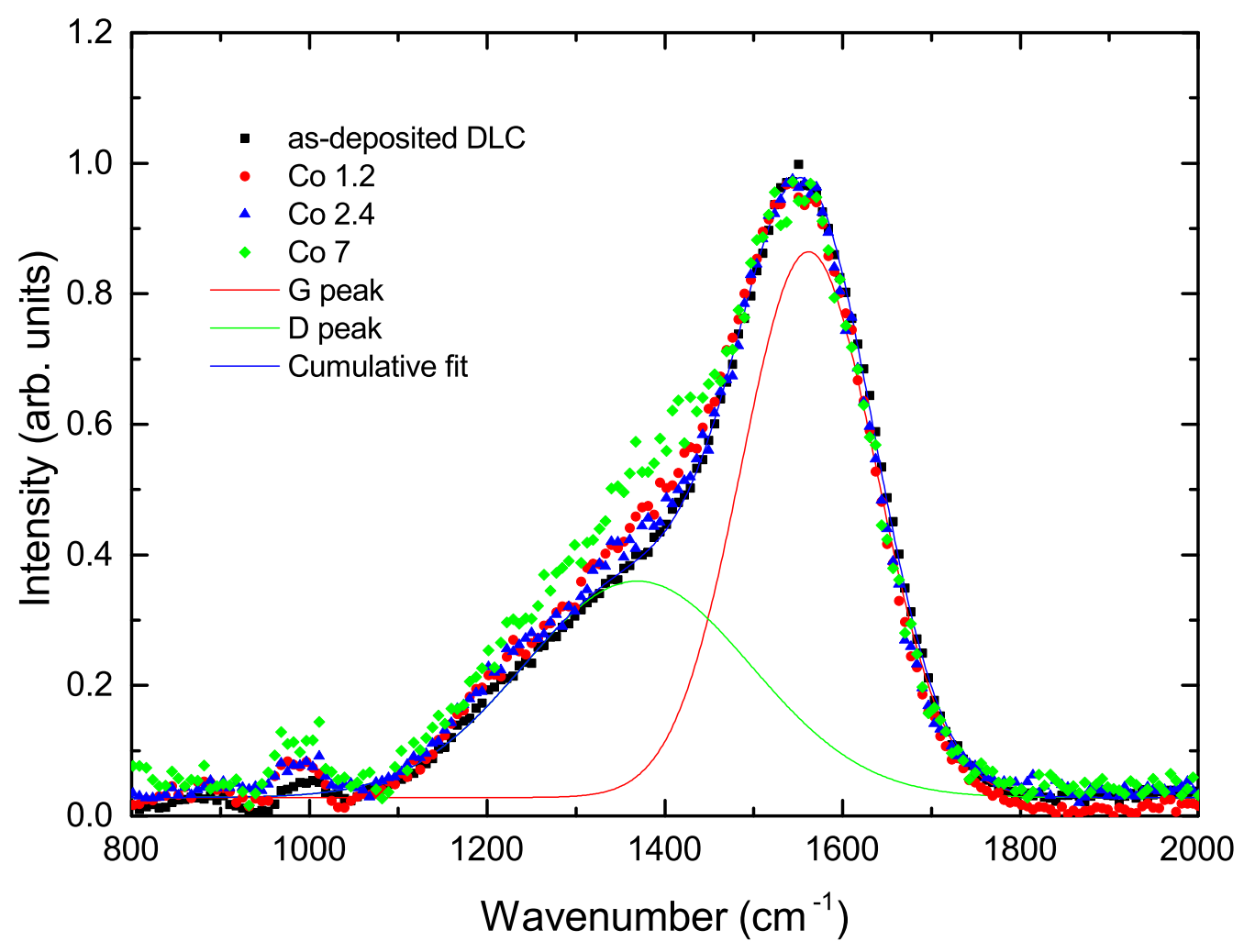

Figure 6.13: Raman Spectrum of as-deposited and Co implanted DLC films (Co 1.2, Co 2.4 and Co 7). The figure shows the spectrum of the as-deposited film fit with $\mathrm{D}$ and $\mathrm{G}$ peak (double Gaussian fit). Changes in the D and G peak parameters with implantation is used to assess the effects of ion implantation on the structure of DLC film.

Three representative samples from the implanted batch were analyzed using Raman spectroscopy. The samples were Co 1.2, Co 2.4 and Co 7 . Raman spectra obtained from these samples are compared against as-deposited DLC in figure 6.13. The as-deposited spectrum was fitted with two Gaussian peaks centered at 1360 and $1562 \mathrm{~cm}^{-1}$. These peaks denote the $\mathrm{D}$ and $\mathrm{G}$ peaks of the film, respectively. The Co implanted films were also fitted similarly with two Gaussians that represents the average D and $\mathrm{G}$ peaks over the implanted region that extends $\sim 60 \mathrm{~nm}$ into the film and the remaining DLC film thickness of $40 \mathrm{~nm}$. The parameters of $\mathrm{D}$ and $\mathrm{G}$ peak used to fit the films are shown in table 6.3. Co implantation has increased the relative intensity of the $\mathrm{D}$ peak and has thus increased the $\mathrm{I}(\mathrm{D}) / \mathrm{I}(\mathrm{G})$ ratio. 
Table 6.3: Fitting parameters of Raman spectra of as-deposited and implanted a:C-H films

\begin{tabular}{|c|c|c|c|}
\hline Sample & G peak position $\left(\mathrm{cm}^{-1}\right)$ & FWHM of $\mathrm{G}$ peak $\left(\mathrm{cm}^{-1}\right)$ & $\mathrm{I}(\mathrm{D}) / \mathrm{I}(\mathrm{G})$ \\
\hline \hline As-deposited & $1562 \pm 2$ & $178 \pm 1$ & 0.67 \\
\hline Co 1.2 & $1560 \pm 2$ & $167 \pm 1.5$ & 1.03 \\
\hline Co 2.4 & $1561 \pm 2$ & $162 \pm 1.5$ & 1.08 \\
\hline Co 7 & $1563 \pm 2$ & $159 \pm 2.5$ & 1.36 \\
\hline
\end{tabular}

Implantation of energetic ions in DLC films result in rearrangement of bonds and atomic displacements of carbon and hydrogen atoms due to transfer of energy from the incoming ions to the DLC matrix. The corresponding effects on the structure of the film can be observed through the changes in the Raman spectra of the implanted films. Raman spectra of the implanted films show a significant rise in the $\mathrm{I}(\mathrm{D}) / \mathrm{I}(\mathrm{G})$ ratio of the films. This can be seen in table 6.3 where the $G$ peak position, FWHM and $\mathrm{I}(\mathrm{D}) / \mathrm{I}(\mathrm{G})$ ratio obtained from two Gaussian fits to the Raman data for the as-deposited and implanted DLC films are shown. This effect is clearer in the plot of the $\mathrm{I}(\mathrm{D}) / \mathrm{I}(\mathrm{G})$ ratio vs. implantation fluence shown in figure 6.14. This indicates that the contribution from $\mathrm{sp}^{2}$ clusters increases with implantation. This could either be due to an increase in disorder or an increase in the number of carbon rings in the DLC film. However the decrease in the FWHM of G peak indicates a reduction in the disorder of the films [10]. This implies that the increase in the $\mathrm{I}(\mathrm{D}) / \mathrm{I}(\mathrm{G})$ ratio is due to an increase in the number of carbon rings. 


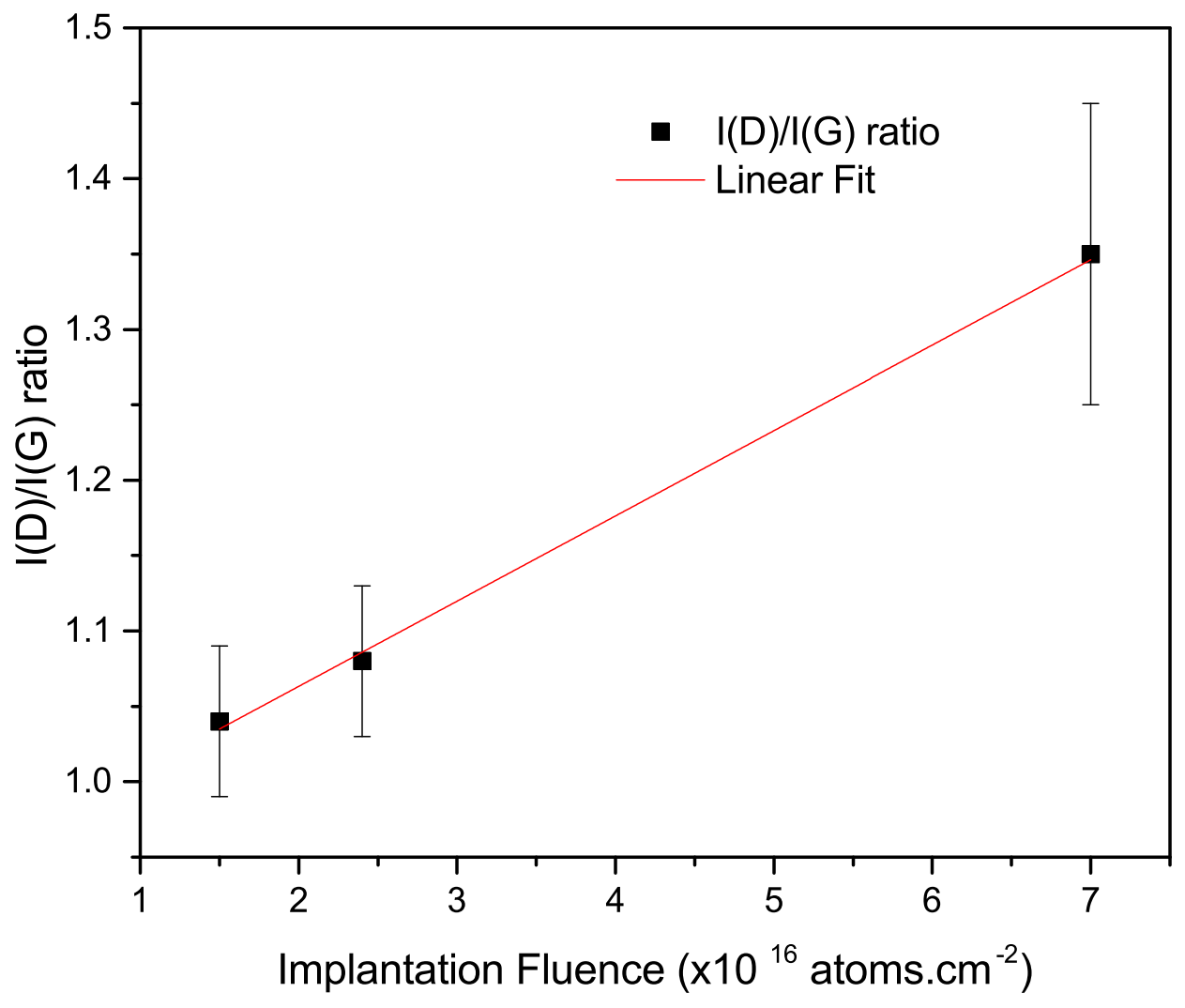

Figure 6.14: $\mathrm{I}(\mathrm{D}) / \mathrm{I}(\mathrm{G})$ ratio plotted as function of implantation fluence. Ion implantation is observed to increase the $\mathrm{I}(\mathrm{D}) / \mathrm{I}(\mathrm{G})$ ratio in proportion with the increase in implantation fluence.

It is to be noted that the $\mathrm{I}(\mathrm{D}) / \mathrm{I}(\mathrm{G})$ ratio will be highest at the region of highest Co concentration and the value measured is averaged over the entire film thickness, e.g. for Co 1.2 implanted DLC film, I(D)/I(G) ratio mentioned contains the contribution from Co implanted region that extends to $60 \mathrm{~nm}$ from the surface (which includes a variation in Co concentration from $0 \%$ to $4.7 \%$ ) and non-implanted region of $40 \mathrm{~nm}$. This suggests that $\mathrm{I}(\mathrm{D}) / \mathrm{I}(\mathrm{G})$ ratio of a DLC film that contains a uniform concentration of Co for example, $4.7 \%$ will have a much higher $\mathrm{I}(\mathrm{D}) / \mathrm{I}(\mathrm{G})$ ratio, nearly reaching 2 . The increase in carbon rings can in turn be due to a number of reasons. Ion implantation can increase the $\mathrm{sp}^{2}$ clustering and aid conversion of carbon from $\mathrm{sp}^{3}$ to $\mathrm{sp}^{2}$ hybridization. However, it can be observed from table 6.3 that the $\mathrm{G}$ peak position is not correlated with the Co concentration or the increase in the $\mathrm{I}(\mathrm{D}) / \mathrm{I}(\mathrm{G})$ ratio. In the three stage model proposed by Ferrari, the $\mathrm{sp}^{2}$ concentration was shown to increase when both the $\mathrm{I}(\mathrm{D}) / \mathrm{I}(\mathrm{G})$ ratio and $\mathrm{G}$ peak position 
changes. Robertson has stated in $[10,8]$ that the non-uniqueness in the relation between the $\mathrm{I}(\mathrm{D}) / \mathrm{I}(\mathrm{G})$ ratio and $\mathrm{G}$ peak position, i.e. similar $\mathrm{G}$ peak position for different $\mathrm{I}(\mathrm{D}) / \mathrm{I}(\mathrm{G})$ ratio or vice versa could be attributed to $\mathrm{sp}^{2}$ clustering without conversion of additional carbon from $\mathrm{sp}^{3}$ to $\mathrm{sp}^{2}$ states. Since the changes in the $\mathrm{G}$ peak position are not significant as shown in table 6.3, it may be the case that the major effect of ion implantation on the DLC structure is increased clustering of $\mathrm{sp}^{2}$ carbon atoms leading to an increase in the short range order and a higher $\mathrm{I}(\mathrm{D}) / \mathrm{I}(\mathrm{G})$ ratio. This clustering is expected to be concentrated in the region that has the highest concentration of implanted atoms.

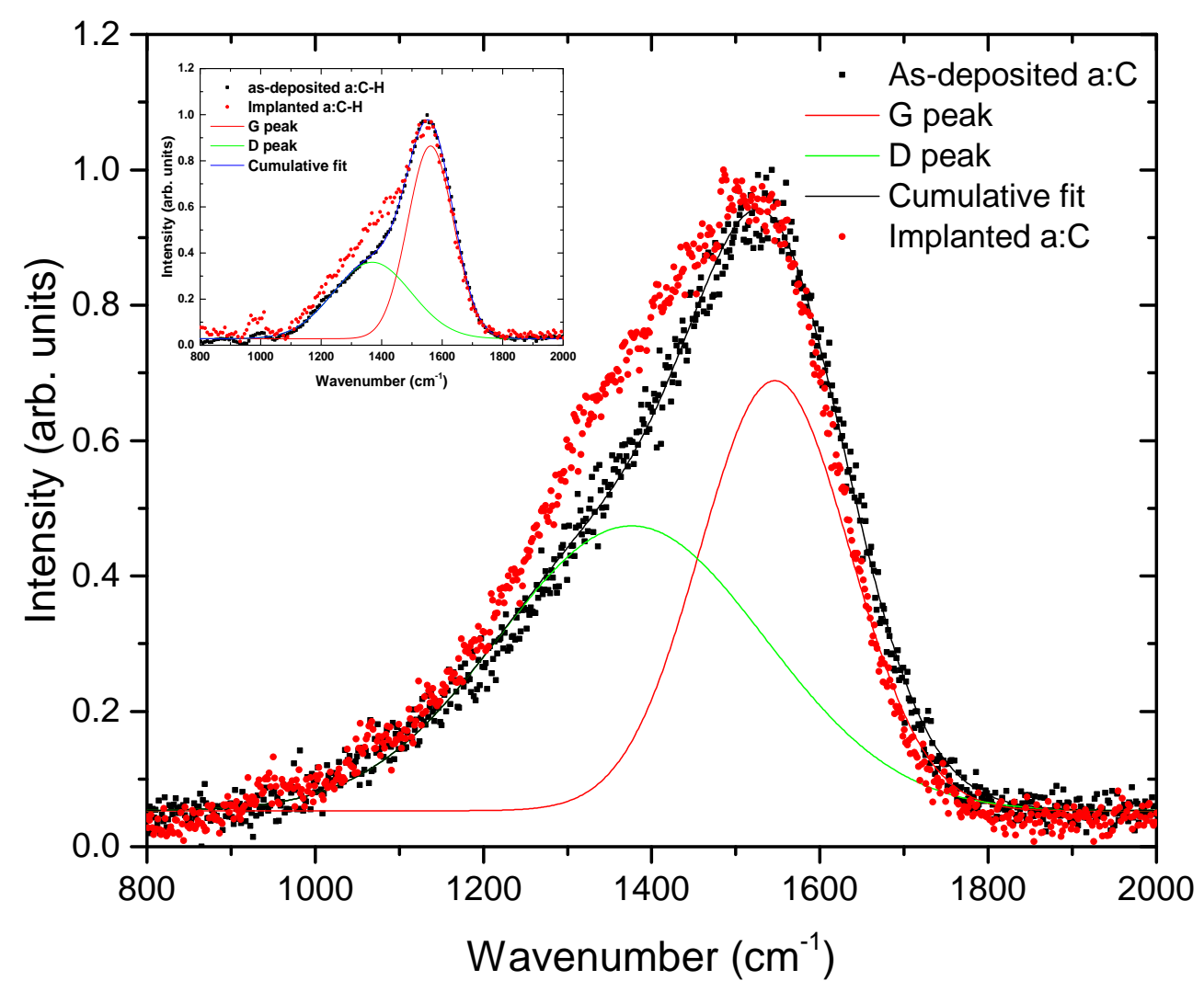

Figure 6.15: Raman spectra of implanted and as-deposited a:C film. The figure shows the Raman spectrum of the a:C films fit with D and G peaks. The Raman fit for a:C-H films are shown in the inset for reference.

One final set of comparison is carried out before concluding the chapter. Raman data from a:C films are compared with the data obtained for a:C-H films. Figure 6.15 shows the Raman spectra of as-deposited and implanted a:C films fit with double Gaussian peaks. The important parameters of the fitting are listed in table 6.4. 
Table 6.4: Fitting parameters of Raman spectra of as-deposited and implanted a:C and a:C-H films

\begin{tabular}{|c|c|c|c|}
\hline Sample & G peak position $\left(\mathrm{cm}^{-1}\right)$ & FWHM of G peak $\left(\mathrm{cm}^{-1}\right)$ & $\mathrm{I}(\mathrm{D}) / \mathrm{I}(\mathrm{G})$ \\
\hline \hline as-deposited a:C-H film & $1562 \pm 2$ & $178 \pm 1$ & 0.67 \\
\hline implanted a:C-H films & $1563 \pm 2$ & $159 \pm 2.5$ & 1.36 \\
\hline as-deposited a:C film & $1545 \pm 3.5$ & $194 \pm 10$ & 1.02 \\
\hline implanted a:C films & $1533 \pm 3.5$ & $186 \pm 14$ & 1.68 \\
\hline
\end{tabular}

Upon implantation in both cases we see an increase in the $\mathrm{I}(\mathrm{D}) / \mathrm{I}(\mathrm{G})$ ratio. As per the Ferrari and Robertson model this may denote an increase in $\mathrm{sp}^{2}$ content and/or cluster size. There is not a significant variation in the $\mathrm{G}$ peak position for a: $\mathrm{C}-\mathrm{H}$ films indicating the variation of the $\mathrm{I}(\mathrm{D}) / \mathrm{I}(\mathrm{G})$ ratio originates predominantly from an increase in cluster sizes of $\mathrm{sp}^{2}$ carbon as concluded before. On the other hand, the variation of the $\mathrm{I}(\mathrm{D}) / \mathrm{I}(\mathrm{G})$ ratio of the a:C film upon implantation is accompanied by a shift in the position of the $\mathrm{G}$ peak. This suggests that the variation is associated with rehybridization of $\mathrm{sp}^{3}$ carbon to $\mathrm{sp}^{2}$ carbon. This difference in the behavior of a:C and a:C-H films upon implantation can be attributed to the presence of hydrogen in the a:C-H films which is known to saturate dangling bonds of carbon preventing them from converting to $\mathrm{sp}^{2}$ hybridization [8].

\subsection{Conclusion}

This chapter presents three main contribution to the thesis. First is the comparison of Co distribution measured in Co implanted amorphous carbon films with hydrogenated carbon. As per the model proposed to explain the bimodal distribution of Co, if the thermal conductivity of DLC is increased by an order of magnitude bimodal distribution should not be observed. This prediction was confirmed to be true in the first section of this chapter. Thus hydrogen plays a key role in determining the Co distribution in DLC. In order to further probe the role of hydrogen in this process, the effect of Co implantation on hydrogen distribution was investigated. This was performed by the use of RNRA technique. The results from this measurements showed several effects which as expected contradicts the DTRIM predictions. The observed features along with the identified deviations are explained by furthering the model used to explain the bimodal distribution. Finally, the chapter was concluded by probing the overall effect of ion implantation on the hybridization and structure of DLC. Raman spectroscopy was used to qualitatively assess the impact of ion implantation. The results suggested that the effect of ion implantation is primarily to enhance the $\mathrm{sp}^{2}$ character of the DLC film. However, based on the presence or absence of hydrogen this effect can manifest in different ways. In hydrogenated DLC, ion implantation primarily increases the cluster 
size of $\mathrm{sp}^{2}$ carbon rings. On the other hand ion implantation into amorphous carbon films, due to lack of hydrogen to saturate the dangling bonds, promotes massive rehybridization of $\mathrm{sp}^{3}$ bonded carbon to $\mathrm{sp}^{2}$ bonding. Thus it is inferred by the detailed investigation that hydrogen plays a prominent role in determining the structure and distribution of DLC films upon ion implantation. 


\section{Chapter 7}

\section{Magnetic and Transport Properties}

\subsection{Introduction}

In this chapter, the magnetic and electrical properties of Co implanted diamond-like carbon films are presented. In the last two chapters, the structure and composition of Co implanted DLC was covered. It was shown that ion implantation into DLC results in unique bimodal distribution with nanoparticle formation. Such distribution has not been observed so far and hence it is of significant interest to measure their magnetic behavior and electrical conductivity.

\subsection{Magnetic Properties}

Magnetic property is the least explored subject in diamond-like carbon systems. Three studies have been identified in literature that deals with the magnetic doping of DLC. The dopants used were $\mathrm{Cr}$ [100], Ni [101] and Co [102]. Co is the preferred magnetic dopant as incorporation of $\mathrm{Co}$ in $\mathrm{TiO}_{2}$, diamond lattice and in some semiconducting materials is believed to lead to dilute magnetic semiconductor [40, 41, 42, 27].

In those studies, $\mathrm{Cr}$ and $\mathrm{Ni}$ doped DLC were synthesized by CVD while Co containing granular carbon films were produced by magnetron sputtering. All these materials were observed to show different magnetic behavior. Chromium doping of DLC was observed to result in ferromagnetic order at low temperature. Increasing the Cr concentration resulted in antiferromagnetic order. The magnetic order was attributed to the carbide nanoparticles formed during synthesis. Ni on the other hand did not interact with the DLC matrix and resulted in Ni nanoparticles. They show room temperature ferromagnetic order and form nanoparticles at concentration as low as 1 at.\%. The magnetic behavior is thus solely attributed to the 
metallic Ni nanoparticles. Cobalt incorporation showed some unique magnetic trends. The incorporated Co formed nanoparticles and exhibited low temperature ferromagnetic order. Interestingly, the magnetization values dropped heavily with temperature but a weak saturated moment was still observed at room temperature. The author attributed the magnetic order to Co particles but unfortunately did not explain the observed temperature dependence. The study did not ascertain the chemical composition of the nanoparticles either. Thus unlike the case of $\mathrm{Cr}$ or Ni doping, the origin of magnetic order in the case of Co doped DLC is not clearly known and can be interesting for magnetic semiconductors.

It is also to be noted that there are no reports on the magnetic properties of magnetic ion implanted DLC in literature. This study explores the origin and nature of magnetic behavior in Co implanted DLC for the first time in literature. 


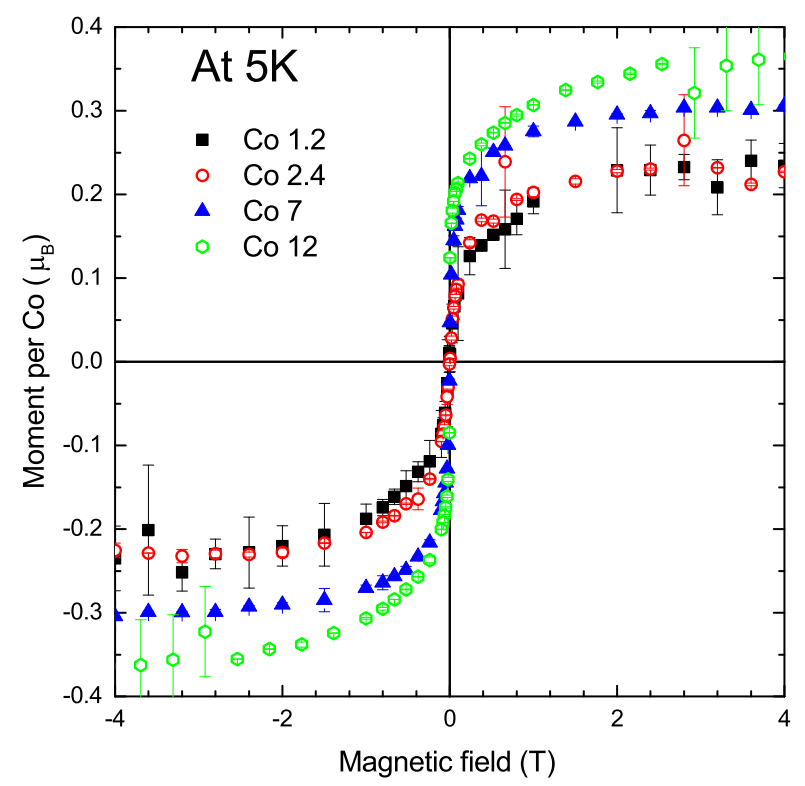

(a) Magnetic moment vs applied Field at $5 \mathrm{~K}$

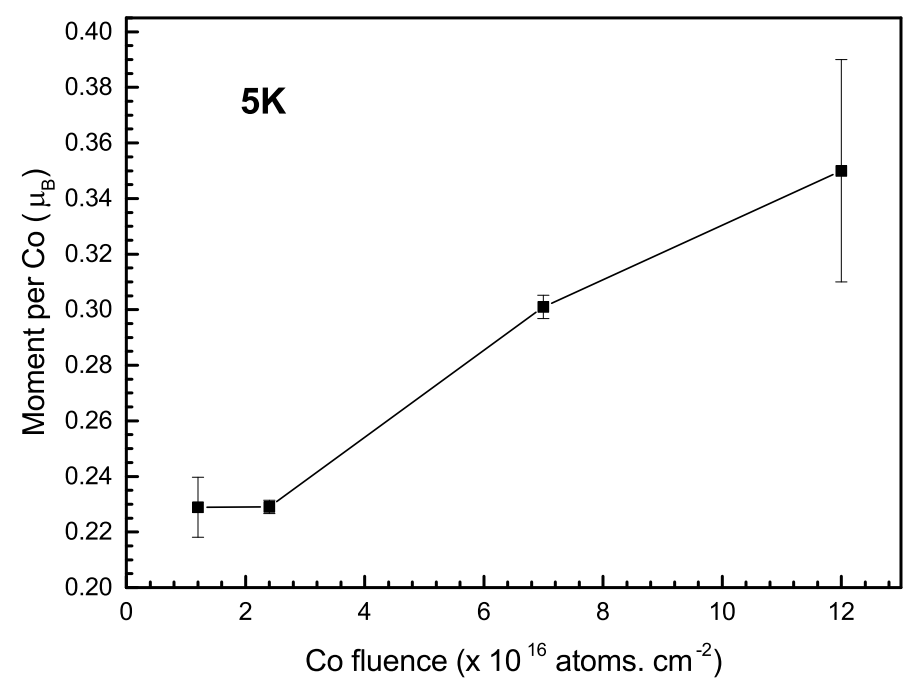

(b) Saturated magnetic moment per Co atom as function of implantation fluence

Figure 7.1: Magnetization measurements of Co implanted DLC (Co 1.2, Co 2.4, Co 7, Co 12 at $5 \mathrm{~K}$ ). 7.1 (a) shows the dependence of magnetic moment induced in Co implanted DLC on the applied magnetic field. The magnetic moment is observed to saturate at fields greater than 2 T. 7.1 (b) plots the saturated magnetic moment per Co atom against implantation fluence. It can be seen that the saturated moment increases with the fluence.

The magnetic moment of DLC films implanted with Co for various fluences were measured under magnetic field sweeps from $-4 \mathrm{~T}$ to $4 \mathrm{~T}$. Figure 7.1 (a) shows the magnetic moment per Co atoms of Co implanted DLC at varying fields measured at $5 \mathrm{~K}$. All samples exhibit 
ferromagnetic order. Hysteresis effects were not observed however small hysteresis loops with coercivity in the order of few tens of Oersted cannot be measured as it is within the noise levels of the instrument. It can be seen that the implanted DLC films starts showing saturation effects as the external field reaches $\sim 2 \mathrm{~T}$. The saturated moment clearly portrays the effect of Co concentration on the magnetic behavior of DLC films. As the concentration of Co in DLC increases the saturated magnetic moment per Co atom increases. It is worth mentioning two points that will be useful in interpreting the above data,

1. The saturated moment is calculated per Co atom. Hence any increase in magnetization occurring merely due to the increase in number of Co atoms is already accounted for in this calculation.

2. The magnetic moment per Co atom measured experimentally or derived theoretically for $\mathrm{Co}, \mathrm{Co}_{2} \mathrm{C}$ and $\mathrm{Co}_{3} \mathrm{C}$ are much higher than that measured in this work. Co as mentioned previously exhibits a magnetic moment of $1.72 \mu_{\mathrm{B}}$ per atom [2]. Measurements on $\mathrm{Co}_{2} \mathrm{C}$ and $\mathrm{Co}_{3} \mathrm{C}$ on the other hand are reported to show values of 1 and $1.81 \mu_{\mathrm{B}}$ per Co atom respectively $[103,104,105]$. Electron diffraction data suggests that the nanoparticles could most probably be $\mathrm{Co}_{2} \mathrm{C}$.

Considering the above factors it is reasonable to expect that only a fraction of implanted Co contribute towards the magnetization of the material. This fraction which is present as nanoparticles is measured to increase with implantation fluence. Cross-sectional analysis by TEM shows that the average nanoparticle diameter increases from $1.2 \mathrm{~nm}$ to $5 \mathrm{~nm}$ as the fluence increases from 1.2 to $7 \times 10^{16}$ atoms.cm $^{-2}$. Furthermore, comparison of Co concentration measured with RBS and the nanoparticle distribution observed in TEM indicate that a significant fraction of Co is diluted within the DLC matrix. If the nanoparticles are assumed to be the major contributor towards the magnetization the increase in its number and size reflects direct increase in the magnetization value.

It is to be noted that the results discussed so far are from magnetization measurements carried out at $5 \mathrm{~K}$. Similar measurements were carried out at higher temperatures and the data for samples Co 7 and Co 12 are shown in figure 7.2 (a) and (b), respectively. Magnetization of samples Co 1.2 and Co 2.4 were also measured at higher temperatures. Their magnetization values dropped significantly and the signal to noise ratio of these data sets are not high enough to gain any meaningful interpretation. 


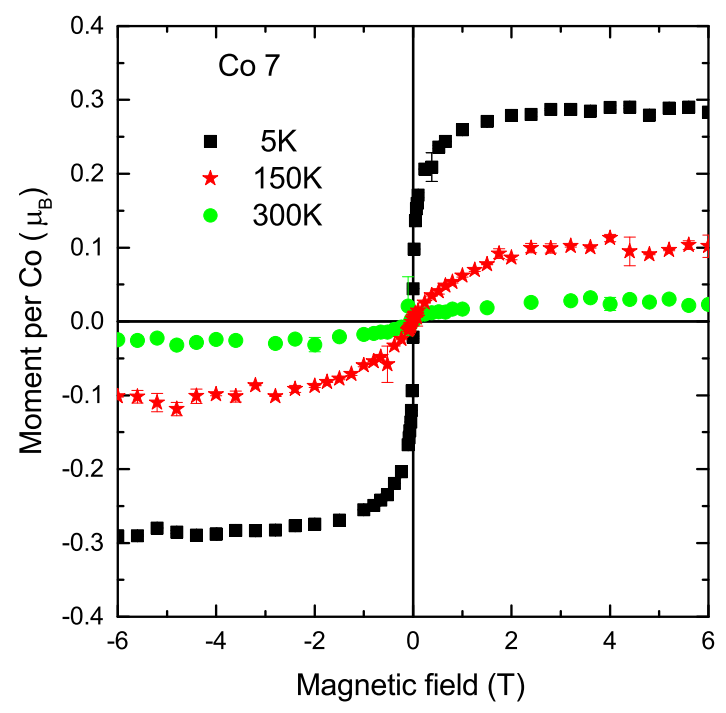

(a) Magnetic moment vs applied field measured for Co 7 at $\mathrm{T}=5 \mathrm{~K}, 150 \mathrm{~K}$ and $300 \mathrm{~K}$

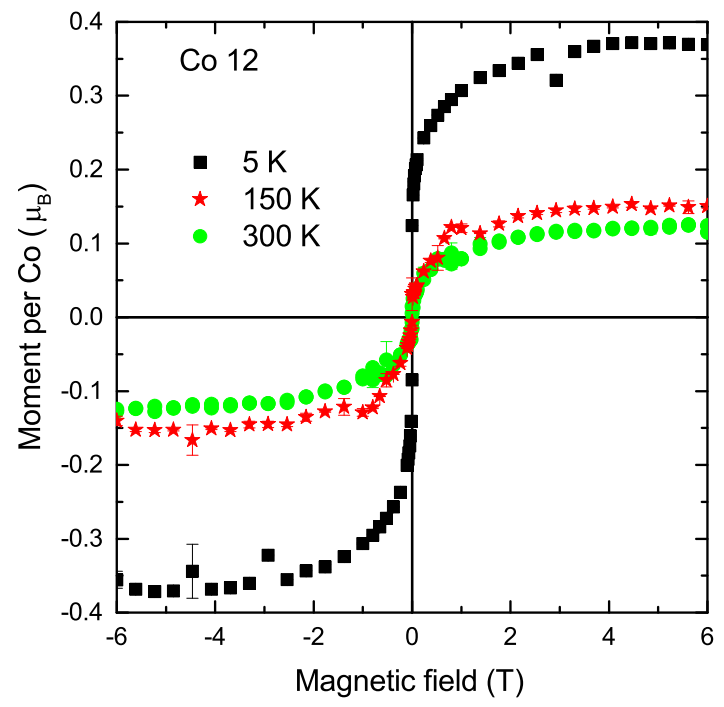

(b) Magnetic moment vs applied field measured for Co 12 at $\mathrm{T}=5 \mathrm{~K}, 150 \mathrm{~K}$ and $300 \mathrm{~K}$

Figure 7.2: Magnetic moment per Co atom measured as function of applied field at different temperatures for sample Co 7 and Co 12. Both sample show strong temperature dependence uncharacteristic of bulk Co.

The figures indicate that the magnetization of the implanted films show strong dependence on measurement temperature. In the case of Co 7 films, the saturated magnetic moment (at $5.6 \mathrm{~T}$ ) drops from $0.29 \mu_{\mathrm{B}}$ to $0.10 \mu_{\mathrm{B}}$ as the temperature is increased from $5 \mathrm{~K}$ to $150 \mathrm{~K}$. The moment further drops to $0.02 \mu_{\mathrm{B}}$ as the temperature is increased to $300 \mathrm{~K}$. 
Overall, increasing the measurement temperature from $5 \mathrm{~K}$ to $300 \mathrm{~K}$ shows a 30 times drop in the saturated magnetic moment per Co atom in Co 7 sample. Co 12 sample shows similar decrease when the measurement temperature is increased from $5 \mathrm{~K}$ to $150 \mathrm{~K}$. The saturated moment per Co atoms drops from $0.37 \mu_{\mathrm{B}}$ to $0.15 \mu_{\mathrm{B}}$, but interestingly a further increase in the measurement temperature to $300 \mathrm{~K}$ results in only a small decrease in the saturated moment $\left(0.15 \mu_{\mathrm{B}}\right.$ to $\left.0.13 \mu_{\mathrm{B}}\right)$.

Earlier the low saturated moment per Co atom was explained by stating that only a fraction of Co is present in nanoparticles. However, a change in measurement temperature cannot result in a change in this fraction (Co present in nanoparticles). Hence there must be other contributing factors that affects the magnetization of the material. In order to investigate this declining trend of magnetization in detail and identify the reason for the different dependency showed by Co 12 when compared to Co 7, the saturated magnetization of both Co 7 and 12 was measured as a function of temperature. Figure 7.3 shows the variation of saturated moment of Co 7 with respect to measurement temperature. The sample was measured from $5 \mathrm{~K}$ to $300 \mathrm{~K}$ at a constant magnetic field of $6 \mathrm{~T}$.

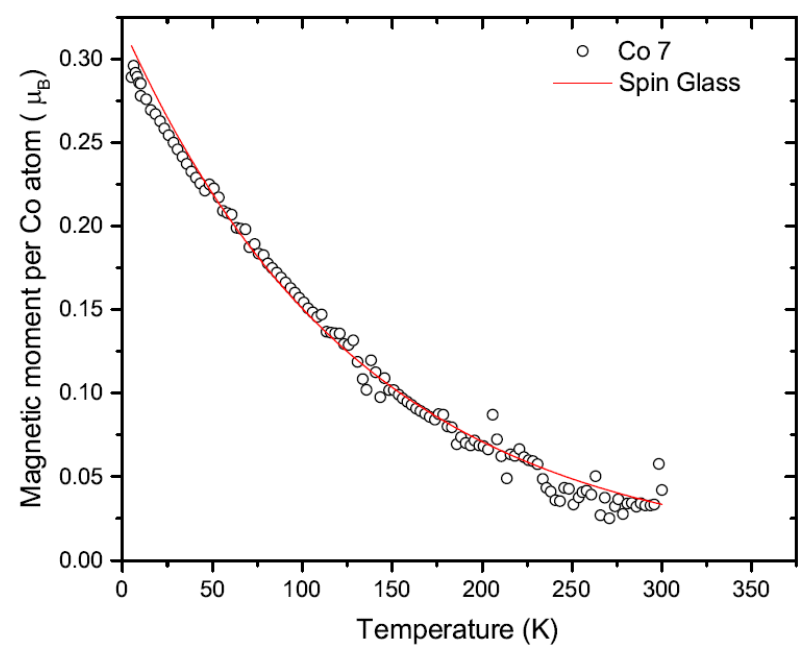

Figure 7.3: Saturated magnetic moment per Co atom measured against temperature for Co 7 sample at a constant applied field of $6 \mathrm{~T}$. The saturated moment falls exponentially with temperature and is fit with a spin glass model with a characteristic spin freezing temperature of $133 \mathrm{~K}$.

The relation between magnetization and measurement temperature shows a unique dependency that is not expected from a ferromagnetic material. As discussed in section 2.2, the effect of thermal energy on a ferromagnet can be explained by spin wave excitations. As the temperature rises the number of such excitations rises which has a detrimental effect 
on the thermally averaged magnetic moment of the material. This variation in magnetic moment of a ferromagnet with temperature can generally be fit with a Bloch function (see section 2.2) of the below form,

$$
m_{s}(T)=m_{s}(0)\left[1-B \times T^{\beta}\right]
$$

where $m_{s}$ represents the saturated moment, $B$ is a constant inversely proportional to spin stiffness coefficient and $\beta=3 / 2$ for Bloch function. The exponent of temperature is usually $3 / 2$, in some cases this exponent is expected to rise upto a value of $2[106,107,108]$. But the trend displayed in figure 7.3 cannot be fit with Bloch function even considering the exponent of $\mathrm{T}$ to reach a value of 2 . Thus the material cannot be characterized as a simple ferromagnet.

A possible explanation could be that multiple magnetic phases are present within the material. Dramatic decrease in saturated moments are usually observed when a material passes its transition temperature, in this case also known as Curie temperature (under the assumption that the observed trend is not characteristic of anti-ferromagnetic behavior). However, figure 7.2 clearly shows that material exhibit room temperature ferromagnetic order. This is evidenced by the saturation of magnetization observed at high fields. Thus if a material undergoes a magnetic transition and still display saturated magnetization it is possible that different magnetic phases present in the sample, with at least one of the phase having a Curie temperature (based on the trend) below room temperature and one of the phase with a Curie temperature above room temperature. But this possibility can be ruled out due to two reasons,

1. If it can be safely assumed that same magnetic phases (though with different compositions) are present then the transition should happen at the same temperature for all fluences. But that is clearly not the case.

2. The magnetization dependence on temperature does not follow a Curie or Curie-Weiss law.

In order to figure out the exact cause for the observed temperature dependence it is necessary to examine the nature in which the magnetic moment drops with temperature. For this purpose figure 7.3 is plotted in a logarithmic scale in figure 7.4. 


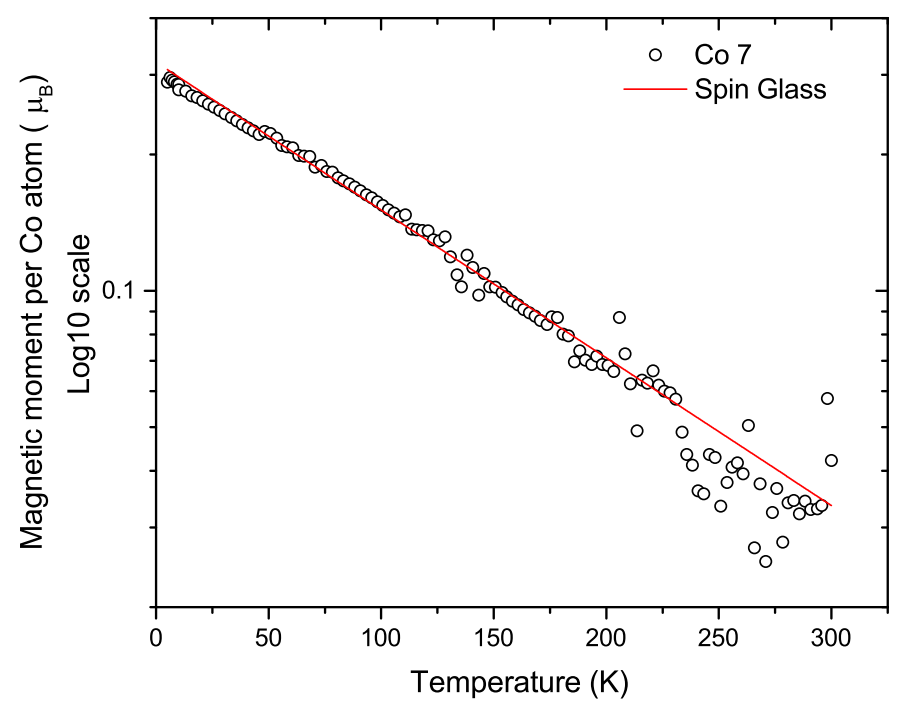

Figure 7.4: Logarithmic plot of magnetic moment vs measurement temperature for Co 7.

In figure 7.4 the saturated magnetic moment per Co atom is represented in the log scale as a function of temperature. It can be clearly seen that the magnetic moment drops exponentially with rise in temperature. A simple ferromagnet or a material with multiple magnetic phases cannot describe such a dependency. From literature, it was found that spin glasses can exhibit such dependency on temperature. Spin glasses are magnetic analogues of glass in material science. Figure 7.5 depicts such a comparison. 


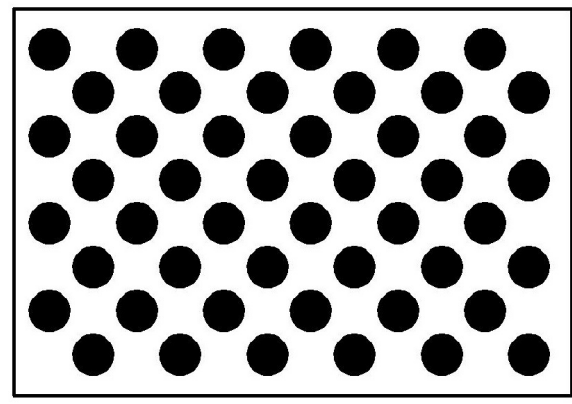

Crystal system

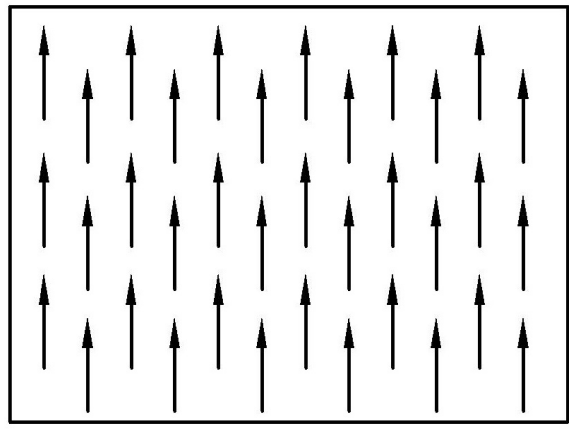

Ferromagnet

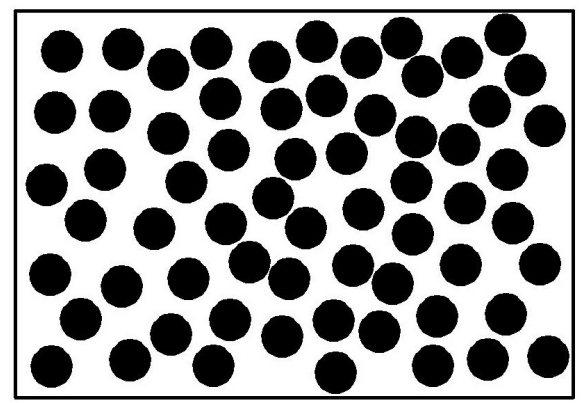

Glass

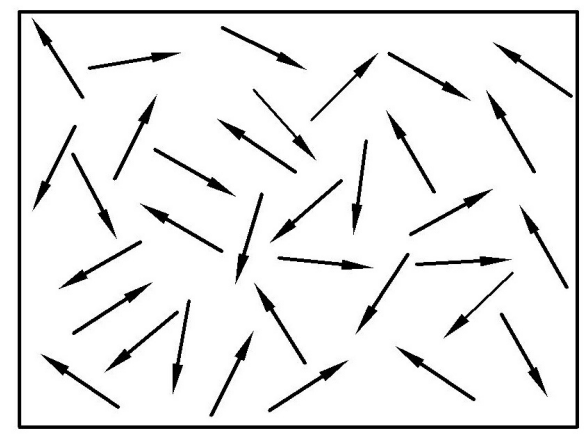

Spin glass

Figure 7.5: Schematic representing crystals - glass / ferromagnets - spin glass.

The phenomenological model based on magnetization of spin glass studied by [109] shows that the effect of temperature can be modeled by,

$$
M_{s}=M_{0} \cdot \exp \left(-\frac{T}{T_{f}}\right)
$$

where $T_{f}$ is denoted as the spin glass temperature. Such behaviors are sometimes observed in magnetic nanoparticles with core-shell structure. The shells are often characterized by presence of high structural or/and spin disorder which can be modeled by spin glass behavior [97]. The spin glass temperature here denotes the temperature below which the disordered spins are frozen in the lattice structure of the spin glass material.

It can be seen from figure 7.3 and 7.4 that the dependence of saturated moment of Co 7 sample on temperature can be fit entirely with a spin glass model. But this is not true for Co 12 sample. It has already been pointed out in figure 7.2 that the temperature dependency of Co 12 is different from Co 7 at higher temperature range ( $150 \mathrm{~K}$ to $300 \mathrm{~K})$. For example, consider figure 7.6, where temperature dependent magnetization of $\mathrm{Co} 12$ is shown in logarithmic scale similar to Co 7 . 


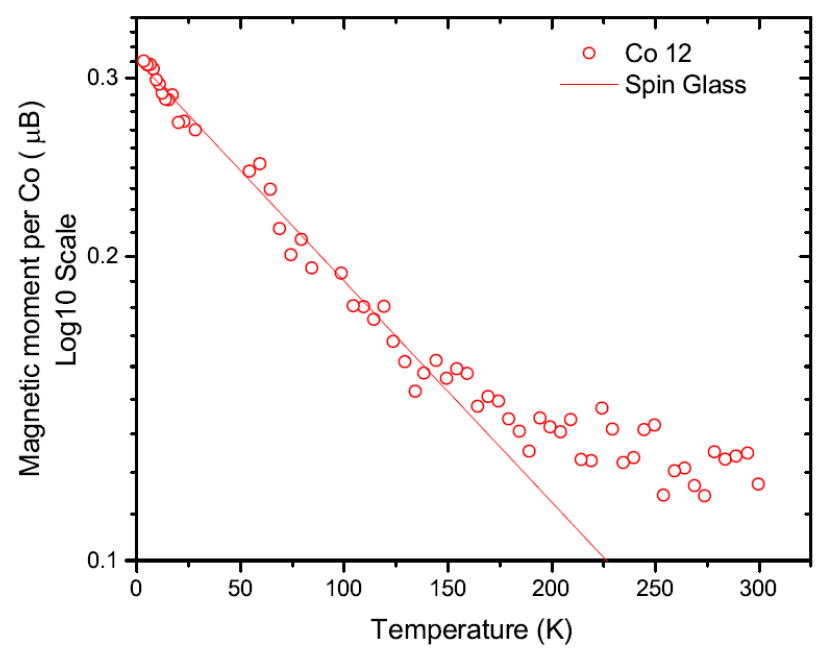

Figure 7.6: Logarithmic plot of magnetic moment vs measurement temperature for Co 12. The saturated moment deviates from the logarithmic dependence at higher temperature $(>$ $200 \mathrm{~K})$. The spin glass contribution was fit with a freezing temperature of $94 \mathrm{~K}$.

It can be clearly seen from the figure that at temperature greater than $150 \mathrm{~K}$ the magnetization trend deviates from an exponential decay expected for a spin glass. The interesting observation in Co 12 is that beyond $150 \mathrm{~K}$, the sample magnetization shows little dependence on temperature. In other words, it behaves as an ordered ferromagnetic material with high Curie temperature. If that is true then this behavior should be modeled by a Bloch function. Figure 7.7 shows the experimental data of Co 12 fit with a combination of functions describing spin glass and an ordered ferromagnet as shown below,

$$
M=M_{s 0} \cdot \exp \left(-\frac{T}{T_{f}}\right)+M_{B 0} \cdot\left[1-B \times T^{\beta}\right]
$$




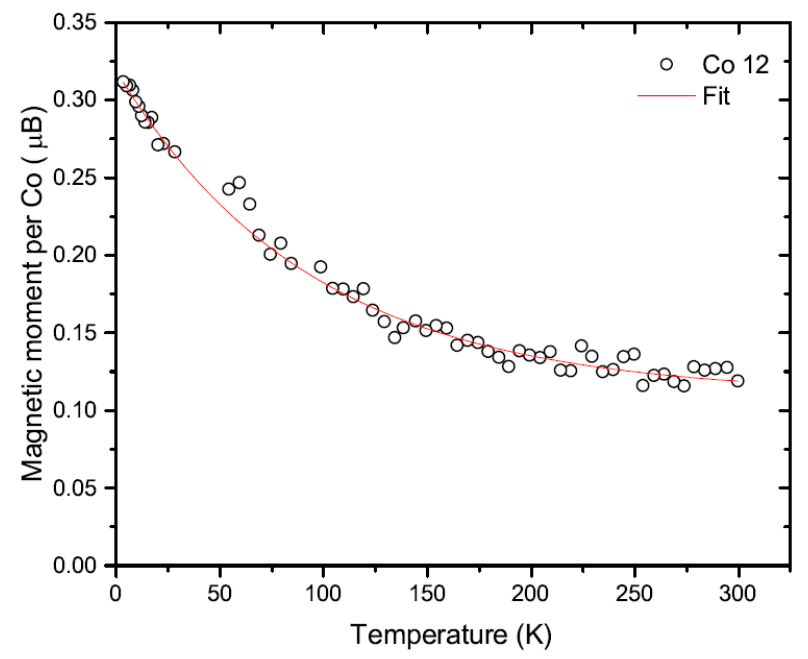

Figure 7.7: Saturated magnetic moment per Co atom measured against temperature for Co 12 sample. The data is fit with a combination of spin glass behavior and Bloch temperature dependence observed in ferromagnets. The spin glass contribution is fit with a spin freezing temperature of $94 \mathrm{~K}$. The Bloch contribution was fit with a spin stiffness coefficient of $1 \times 10^{6}$.

From the fit, the spin glass contribution to the magnetization is derived to be $0.20 \mu_{\mathrm{B}}$ at absolute zero which is only two third of the total magnetic moment. Recall that the spin glass behavior in nanoparticles is in general attributed to the structural disorder of the shell structure surrounding the nanoparticles. A reduction in spin glass component would thus indicate an increase in the structural order of the nanoparticles. This leads to the ferromagnetic order within the material. If this model is true then such an increase in structural order should reflect in the TEM data. Further investigation in this track confirms this model. 


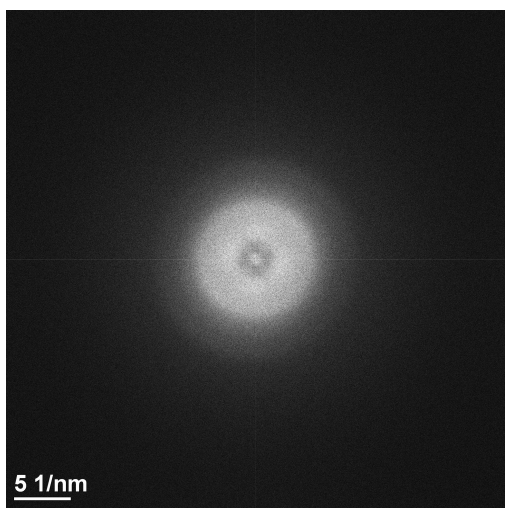

(a) FFT of HR-TEM image of Co 1.2

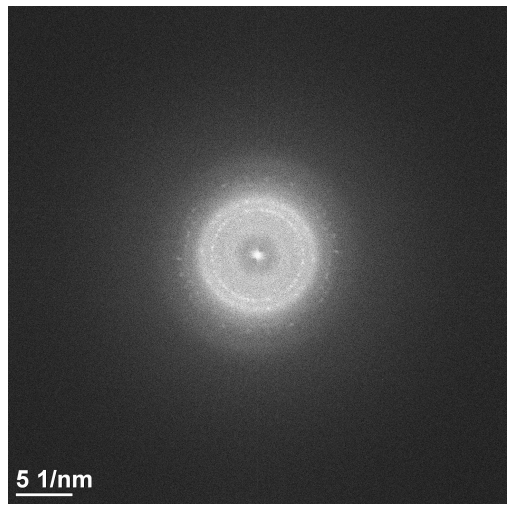

(c) FFT of HR-TEM image of Co 7

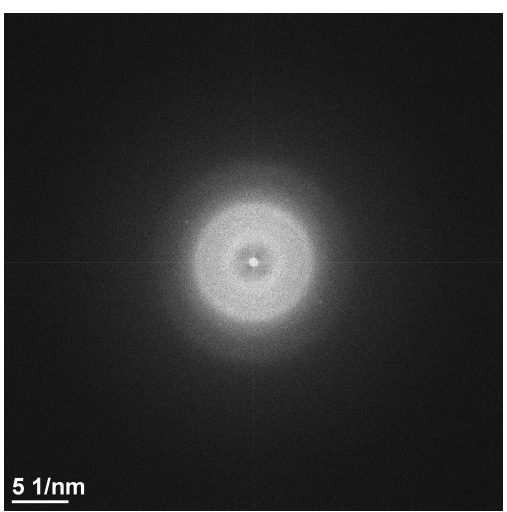

(b) FFT of HR-TEM image of Co 2.4

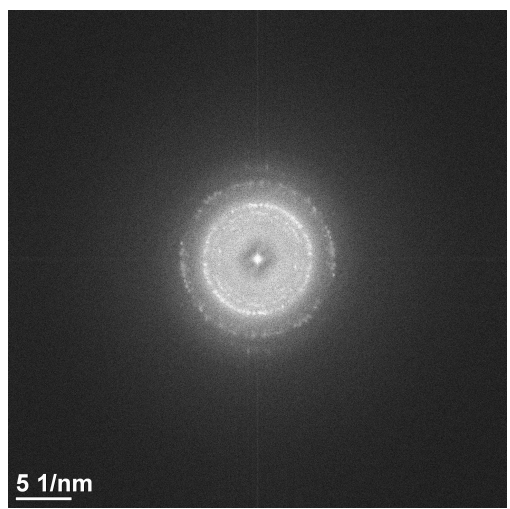

(d) FFT of HR-TEM image of Co 12

Figure 7.8: FFT of HR-TEM images of Co implanted DLC reflecting the structural order present within the nanoparticles.

Figure 7.8 shows the FFT data from all the four samples discussed in this section. It can be seen that for Co 1.2 and Co 2.4 there is no significant ordering observed in the material. As the fluence increases, for Co 7 sample a pattern reflecting possible crystal structure within the nanoparticle emerges. In figure 7.8 (d) representing sample Co 12, the pattern gains greater clarity and ring-like structures in the reciprocal space can be identified to specific dspacings of carbide systems. This indicates the trend observed with increasing implantation fluence. Co 12 shows a significant improvement in the crystallinity of the material. This is confirmed by the results obtained from the magnetic measurement.

So far several interesting features about magnetic nanoparticles produced by ion implantation in DLC was discussed. The magnetization is said to occur primarily from the magnetic nanoparticles. This can be confirmed by probing the magnetization of the material as a function of temperature at low magnetic field. In section 2.2 features of superparamagnetism observed in nanomaterials were discussed. A characteristic feature of such materials is the observation of irreversibility at the temperature region below the blocking temperature of the 
nanoparticles. In these materials the magnetocrystaline anisotropy energy (MAE) is comparable to the thermal energy and at the temperature where the thermal energy overtakes the MAE, is referred to as blocking temperature.

Measuring the magnetic moments of the samples at low field strengths during field cooling and zero-field cooling allows probing the irreversibility of the sample [110]. ZFC-FC measurements help computing the magnetocrystaline anisotropy energy of the sample which is usually determined to compute the size of the nanoparticles. However, in this case the ZFC-FC measurements cannot be directly applied to compute the particle size due to the effects of structural disorder leading to spin glass behavior. Figure 7.9 and 7.10 shows the ZFC-FC curve obtained for sample Co 7 and Co 12 respectively.

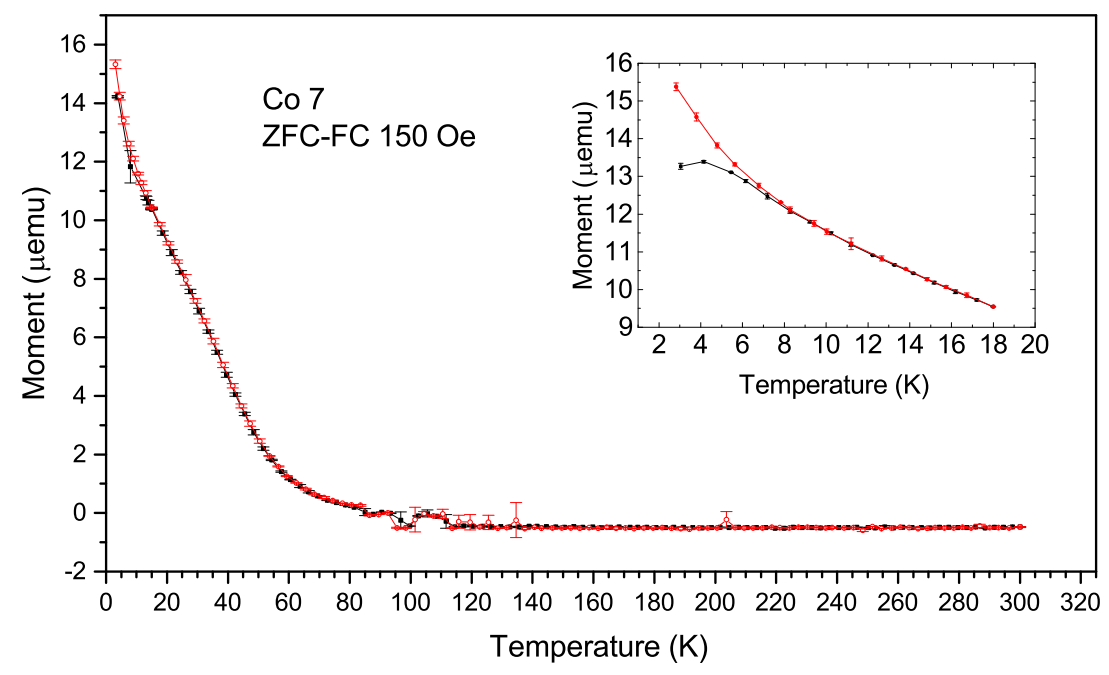

Figure 7.9: ZFC-FC measurement performed on Co 7 at $0.015 \mathrm{~T}$. The inset shows the splitting between the FC and ZFC curves at $4 \mathrm{~K}$ indicating superparamagnetic behavior. 


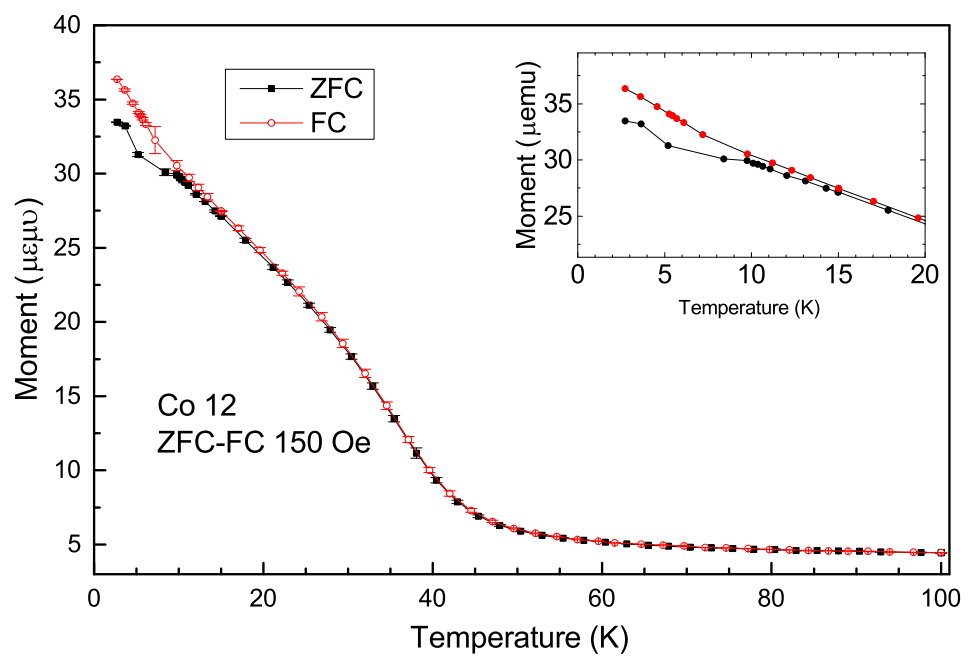

Figure 7.10: ZFC-FC measurement performed on Co 12 at $0.015 \mathrm{~T}$. The inset shows the splitting between the FC and ZFC curves. The splitting occurs at a larger temperature than observed for Co 7 indicating superparamagnetism from larger nanoparticles.

The measurement was carried out at $0.015 \mathrm{~T}$ during field cooling and zero-field cooling. For Co 7, the measurement was carried out from $3 \mathrm{~K}$ to $300 \mathrm{~K}$, while in the case of Co 12 the measurement was carried out from $3 \mathrm{~K}$ to $100 \mathrm{~K}$. In both the measurements the irreversibility was obtained at very low temperature. The insets in the figure show the splitting of $\mathrm{FC}$ and ZFC magnetization curves (was carried out as a separate measurement). This indicates the temperature at which irreversibility is observed. In the case of Co 7, the splitting of the magnetization curves beyond the experimental uncertainty was observed starting from $6 \mathrm{~K}$ and below. On the other hand, in the case of Co 12 irreversibility was observed to manifest starting from $12-14 \mathrm{~K}$.

Measuring the blocking temperature allows computing the particle size [110],

$$
T_{B}=k V / 25 k_{B}
$$

In the above equation, $\mathrm{k}$ is the anisotropy constant and is dependent on the material and its crystal structure. Literature suggests that $\mathrm{Co}_{3} \mathrm{C}$ has an anisotropy coefficient of $7.5 \times 10^{5}{\mathrm{~J} . \mathrm{m}^{-3}}^{-3}$ [104]. The anisotropy constant of $\mathrm{Co}_{2} \mathrm{C}$ is unclear however it was suggested in [111] that the anisotropy of cobalt carbides ranges from $6.5-9 \times 10^{5} \mathrm{~J}^{-\mathrm{m}^{-3}}[111,104]$ and is higher than that of bulk Co which has an anisotropy of $2.5 \times 10^{5} \mathrm{~J}^{-\mathrm{m}^{-3}}[2,112]$. Computing the particle size for the above temperature ranges provides a particle size (diameter) ranging 
from 1.64 to $1.82 \mathrm{~nm}$ for carbides and $2.51 \mathrm{~nm}$ considering the anisotropy coefficient of Co for Co 7 sample. Similarly for Co 12 the particle size was calculated to be 2.17 to $2.43 \mathrm{~nm}$ for carbides and $3.33 \mathrm{~nm}$ for Co.

There are two factors that needs to be considered while interpreting the particle size. First, as revealed from TEM the samples contain a range of particle sizes and the absence of well defined peak in the ZFC-FC measurement indicate the presence of a range of blocking temperature. Thus the computed particle size does not represent the whole sample but rather only a fraction of nanoparticles. An even more important consideration is the disorder present in the nanoparticles. The disordered regions due to their random arrangement will not have the same MAE of the ordered phase. Thus even though the nanoparticles are observed to be superparamagnetic they do not resemble any well defined systems of cobalt or cobalt carbides as reported in literature.

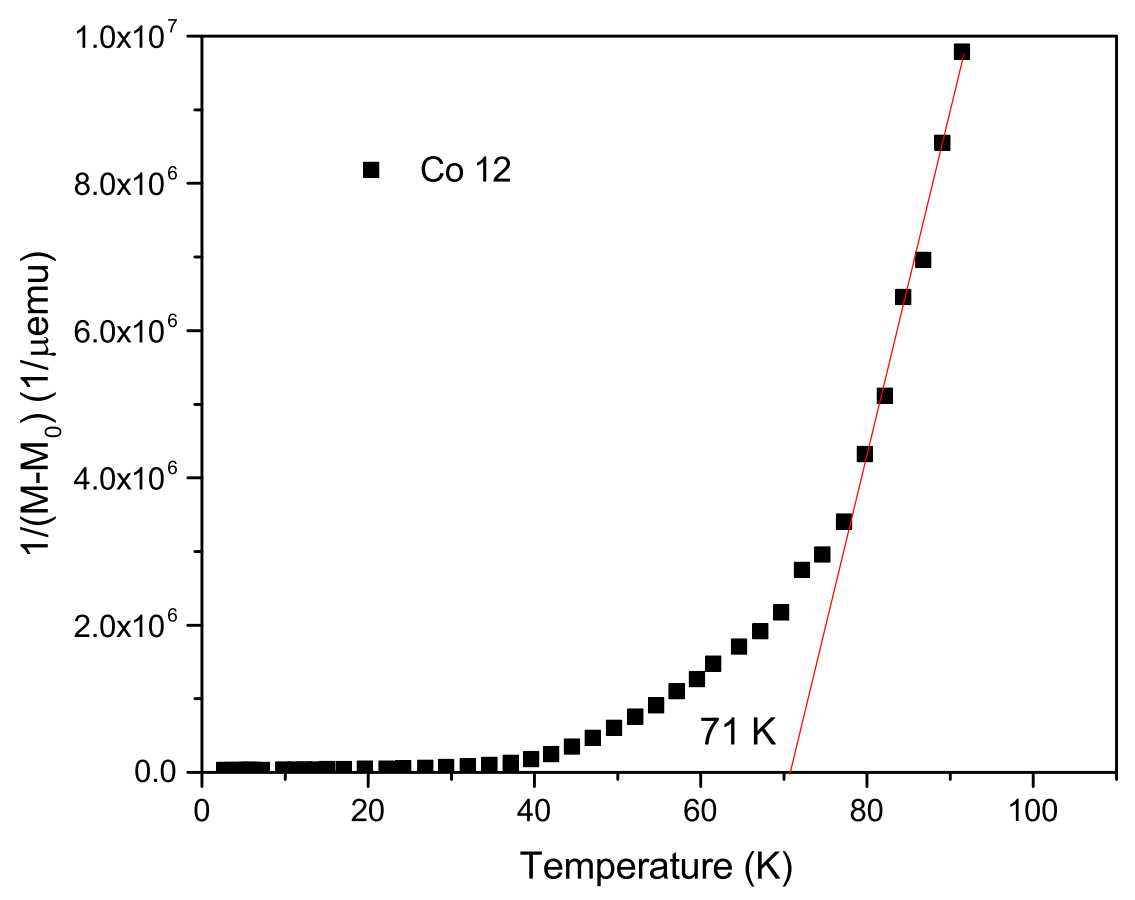

Figure 7.11: High temperature region of ZFC-FC measurement performed on Co 7 at 0.015 $\mathrm{T}$. The inverse of magnetic moment is plotted against measurement temperature showing the non-Curie dependence of the superparamagnetic nanoparticles.

ZFC-FC measurement on Co 12 provides another interesting property of the Co implanted DLC. It was mentioned in section 2.2 that magnetic nanoparticles that are superparamagnetic in principle can be fit with Curie law at temperature well above their blocking 
temperature. This is valid only for those systems where the nanoparticles are well-separated and do not interact with each other. In such systems, the magnetic moment dependency on temperature is given by the Curie relation described in section 2.2. The magnetic moment of the nanoparticles (total moment subtracted of the contribution from the substrate) is thus related to the inverse of temperature,

$$
M-M_{0} \propto \frac{1}{T}
$$

The relation can be rewritten to exhibit the dependency on temperature as,

$$
\frac{1}{M-M_{0}} \propto T
$$

The expression suggests that the intercept of $1 /\left(M-M_{0}\right)$ curve on T should pass through 0 . However, it can be seen from the plot that the curve makes a positive intercept which implies a Curie-Weiss behavior as given below,

$$
\frac{1}{M-M_{0}} \propto(T-\theta)
$$

where $\theta$ represents the Weiss temperature. The fit of the above data gives a positive Weiss temperature. This is surprising as in a nanoparticle system, the dipole-dipole interaction between the particles if present is expected to cause a reduction in the overall moment . However, the positive Weiss temperature for Co implanted DLC suggests the dipole interaction of the nanoparticles to result in an enhancement of the overall magnetization of the sample. This is an interesting result that is not reported previously in such systems. This interaction could arise from the double layered distribution of magnetic nanoparticles, where the disordered phases can aid in the enhancement of the ordered ferromagnetic phase of the large nanoparticles.

The magnetic results so far indicate that magnetic ion implantation into DLC leads to several interesting properties and new phenomenon that has not been reported so far.

\subsection{Electrical conductivity}

As deposited DLC films exhibits an electrical resistance in the order of 1 - 100 gigaohms when measured by a four probe method with an electrode separation of $1 \mathrm{~mm}$. Conduction in these films are described well by variable range hopping mechanism. Ion implantation is known to reduce the resistance significantly. This reduction was found to be directly proportional to the implantation dose [38]. The reduction can have two possible causes 
for occurring. The first cause could be due to the enrichment of the $\mathrm{sp}^{2}$ character of the DLC films. In the previous chapter, it was shown that ion implantation in hydrogenated diamond like carbon, increases the size of the $\mathrm{sp}^{2}$ clusters. Since the electrical conductivity is determined by the $\mathrm{sp}^{2}$ carbon, an increase in cluster size can result in similar increase in conductivity. The other possible cause for the reduction in resistance can be attributed to the formation of metallic nanoparticles $\left(\mathrm{Co}_{2} \mathrm{C}\right.$ and $\mathrm{Co}_{3} \mathrm{C}$ are metallic) within the implanted region of the DLC films. These metallic precipitates can open up new conduction pathways with relatively lesser resistance to the passage of charge carries. 


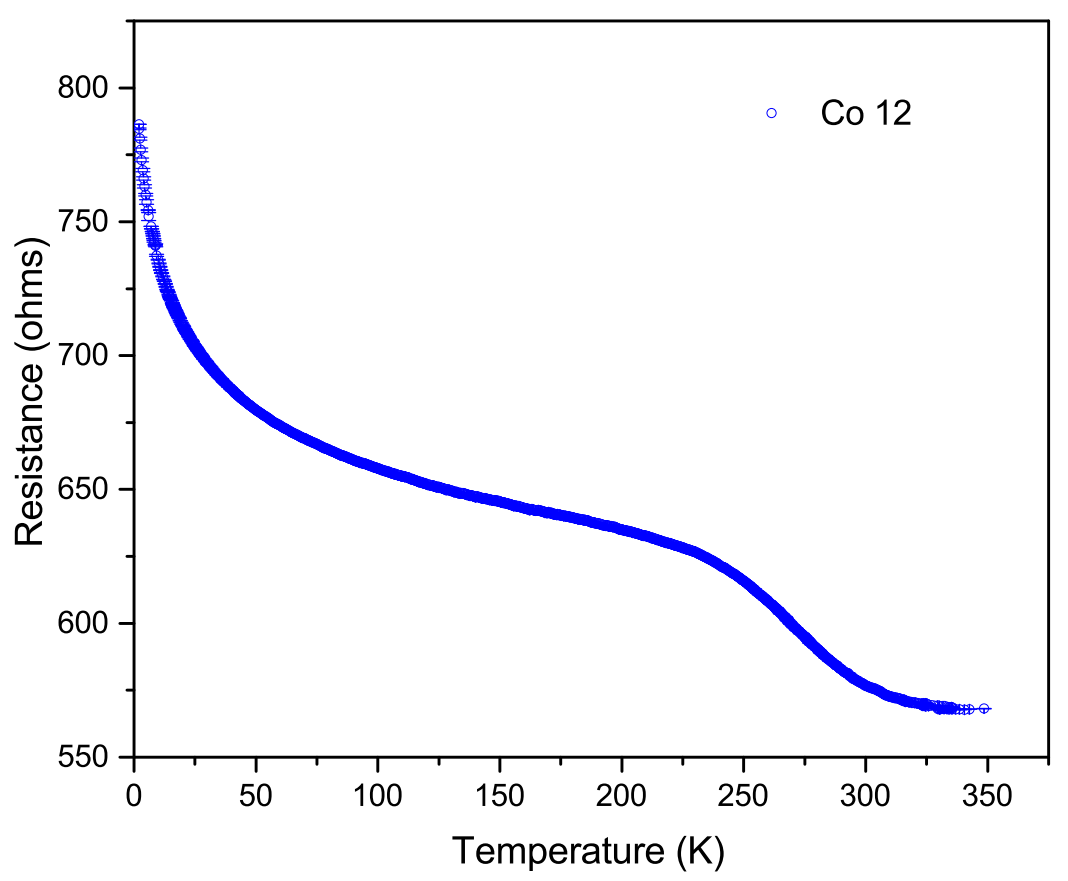

(a) Resistance vs temperature plot for Co 12

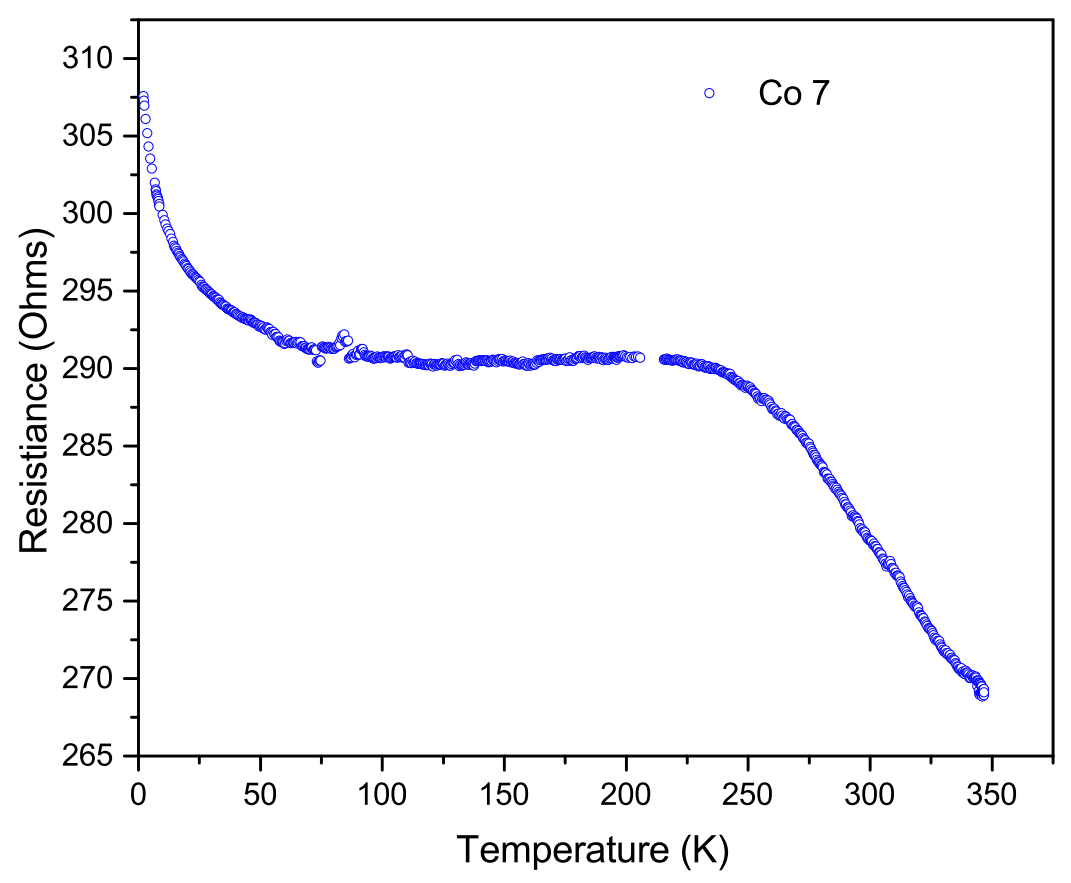

(b) Resistance vs temperature plot for Co 7

Figure 7.12: Resistance measured against temperature for sample Co 12 and Co 7. Both Co 12 and Co 7 show decrease in resistance with increasing temperature. However, the data curve indicates that the temperature dependence is not uniform throughout the temperature range measured. 
Figure 7.12 shows the change in electrical resistance with respect to the measurement temperature for samples Co 7 and Co 12. It can be seen from the figure that the electrical resistance has dropped nearly by a factor of $10^{6}$. This drastic reduction does not correspond directly to the enrichment of $\mathrm{sp}^{2}$ phase of DLC. It is to be further noted that these results strongly indicate that the electrical conduction happen through the implanted region of the DLC films as conduction through non-implanted region will experience an electrical resistance in the order of Gohms.

In a previous work [38] DLC films were implanted with transition metal ions and noble gas ions. Both these implantation caused a reduction in electrical resistance. However, it was shown that the transition metal implantation caused $10^{3}-10^{4}$ times greater reduction in electrical resistance compared to the noble gas ions. These evidence strongly suggest that the dominant cause for reduction in electrical resistance is the formation of metallic precipitates in the implanted region of DLC. It is to be noted that in this case, both of the above mentioned factors ( $\mathrm{sp}^{2}$ enrichment and metallic precipitates formation) can occur simultaneously. However, the conduction mechanism is predominantly affected by the presence of cobalt containing nanoparticles. Hence, the data presented above will be interpreted from the perspective of conduction through metallic nanoparticles.

Referring back to the data shown in plots, few definite points can be made about the transport properties of implanted DLC films. The first inference from the data is that the sample displays a negative temperature coefficient for the electrical resistance. This shows that the sample do not exhibit a metallic behavior, which implies that the nanoparticles do not combine to form an interpenetrating network. The second inference can be made from the nature / trend of reduction in resistance with increase in temperature. The data shows that the drop in resistance is neither linear nor uniform throughout the temperature range. The resistivity drops in a particular trend up to a certain point of temperature (200 - $250 \mathrm{~K})$. An increase in temperature beyond the point witness a deviation in the trend for resistance drop.

Consider the temperature dependence of resistance in the low temperature region for sample Co 12. 


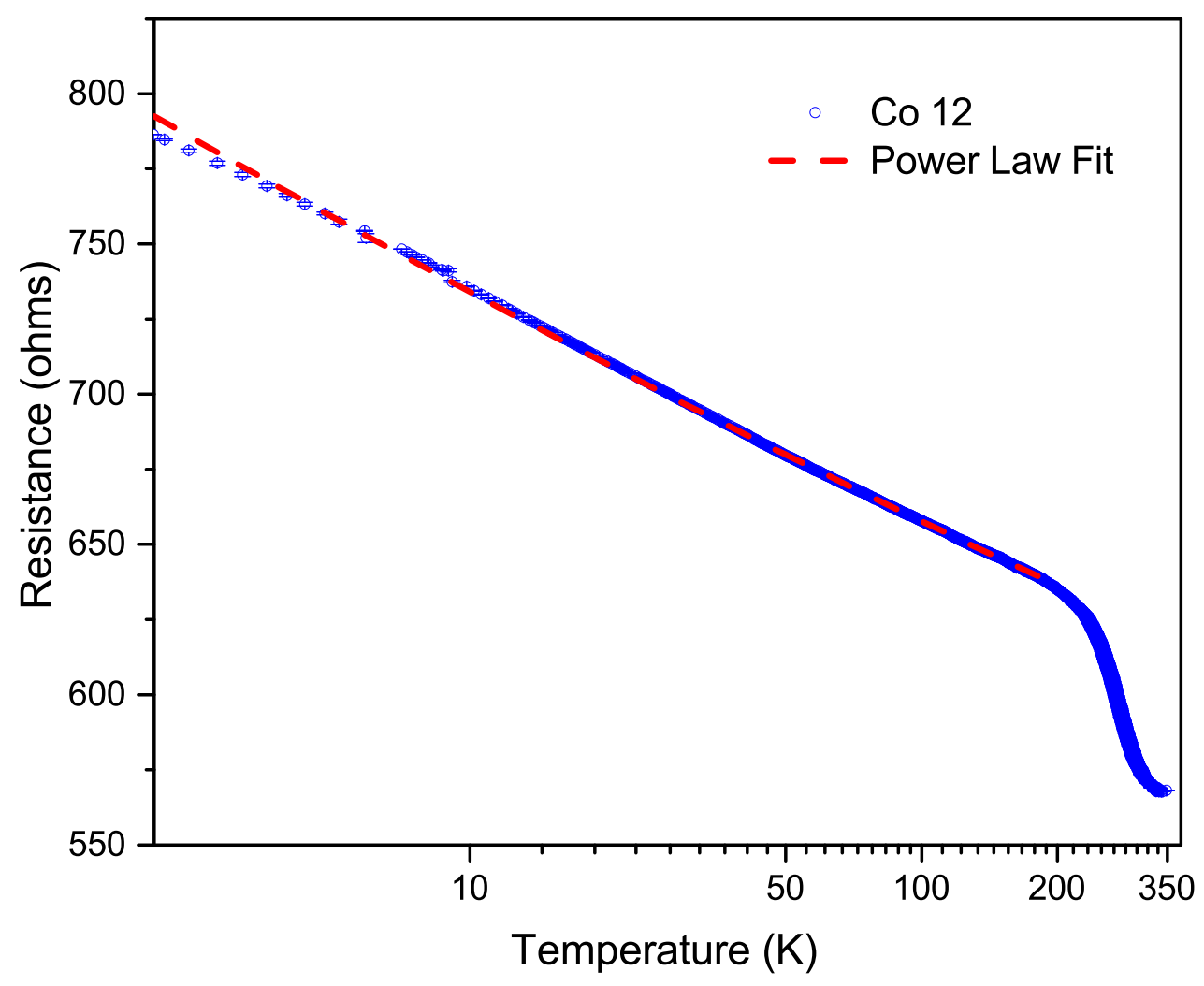

Figure 7.13: Logarithmic temperature dependence of resistance of Co 12 in the low temperature region.

Figure 7.13 shows the resistance data of Co 12 in $R$ vs Log $(T)$ plot. The temperature dependence do not correspond to thermal activation, variable range hopping or hopping conduction as non of them show a logarithmic temperature dependence. One possible way to explain the observed temperature dependence is by considering the thermally assisted tunneling between the large nanoparticles (as they contain the ordered phase). In a well defined system of nanoparticles with constant barrier height and width, thermal energy contributes towards lowering of the energy barrier and the resistance of the sample exhibits an exponential dependence over temperature. However, in Co implanted DLC the nanoparticles are distributed randomly in both size and position. The barrier height, which is dependent on the nature of DLC separating the nanoparticles and the barrier width determined by the distance separating the nanoparticles can assume a wide range of values. A realistic representation of such a system would require a 3D network of resistor chains, with each resistor representing the tunnel resistance between any two nanoparticles of the system. 
Each of this resistance can have different dependency on temperature. Such a system is inhomogeneous in nature and can show any kind of temperature dependency. Thus it is extremely complex to model.

A simple first order approximation in such a scenario is to describe the dependence of resistance on temperature as a weak power law fit as given below,

$$
R_{t}=R \cdot T^{m}+C
$$

where $R_{t}$ represents the resistance at temperature $T, R$ represents the resistance at $0 \mathrm{~K}$ and $\mathrm{C}$ is a constant. The power factor $m$ represents the average strength of the temperature dependence of the tunnel resistance considering a range of barrier heights and widths between nanoparticles.

As mentioned previously, the power law dependence is observed only in the low temperature region. At higher temperature the behavior deviates slightly. This deviation suggests an additional contribution which can be modeled as a parallel pathway for electrical conduction. Since the conductivity is limited to the implanted regions of the DLC film the contribution could arise from the region containing the smaller nanoparticles. This is represented as a schematic in figure 7.14 . 


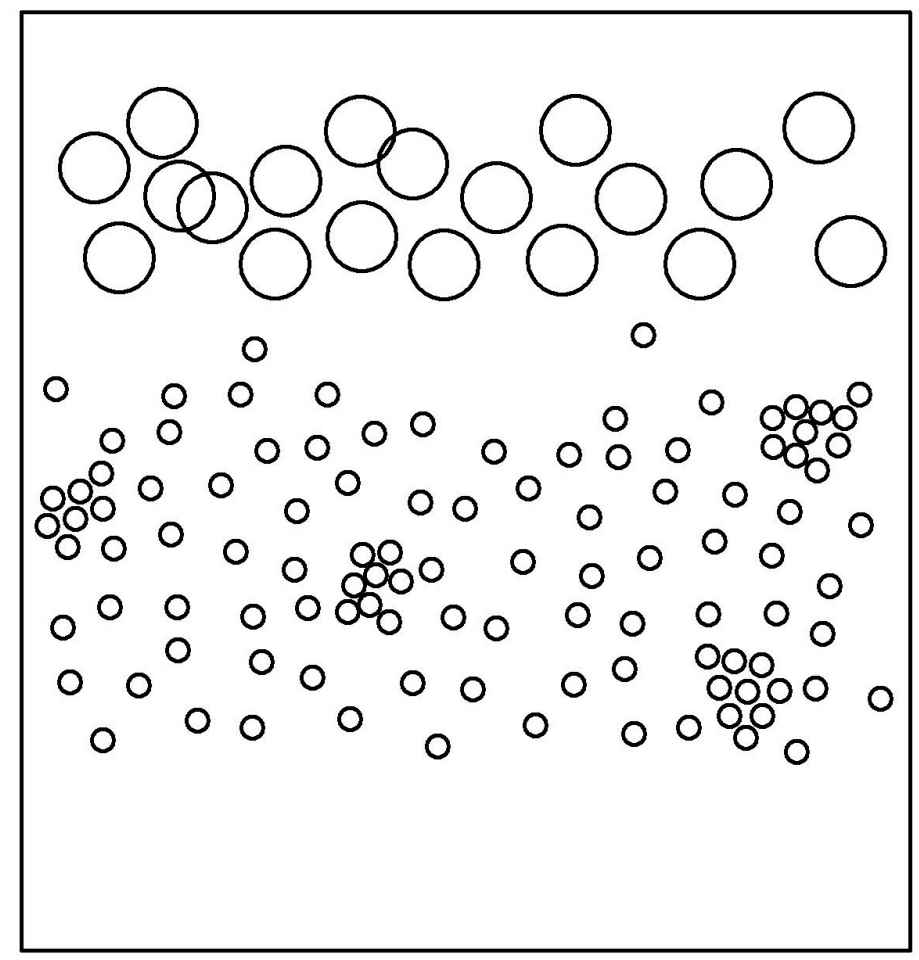

(a) Transparent structure in DLC

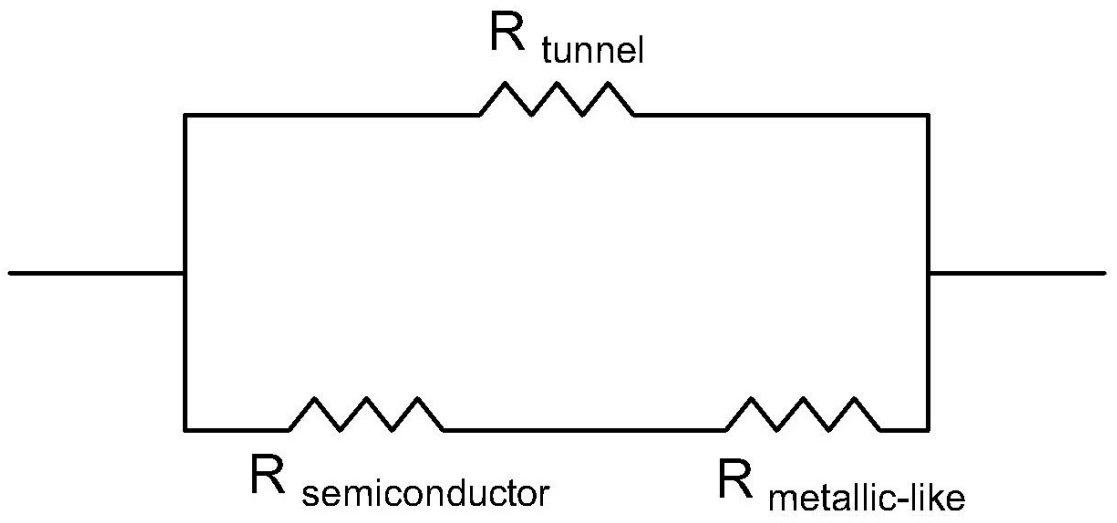

(b) Resistant network

Figure 7.14: Schematic representing the simplified model of conduction mechanism in Co implanted DLC. The conduction through the implanted DLC region can be split into two parallel conducting chains with tunneling resistance dominating at low temperature region (forming the first chain) and the semiconducting and metallic contribution to conduction at higher temperature region (forming the second parallel chain).

Depending on the spatial density of the small nanoparticles, the contribution of resistance from the region can be explained by thermal activation or scattering effects. When the nanoparticles are sufficiently apart the conduction can happen through hopping between the 
$\mathrm{sp}^{2}$ clusters of the DLC film given by,

$$
R_{s}=r_{s .} e^{\left(\frac{D}{T}\right)}
$$

which resembles a contribution from a semiconductor. Here, $R_{s}$ represents the resistance contribution from the semiconducting resistance $r_{s}$ at temperature $T$ with an activation barrier of $D$ represented in temperature scale.

When the nanoparticles are closely positioned so as to form a percolation pathway, increase in temperature can lead to scattering effects which in turn increases the resistance of the region. This is given by,

$$
R_{m}=r_{m} T^{n}
$$

where $R_{m}$ represents the resistance contribution from metallic regions $\left(r_{m}\right)$ at temperature $T$ and $n$ represents the positive power factor of temperature dependence.

Thus the overall conduction mechanism in Co 12 can be explained by a model which simplifies the complex multiple pathways of conduction in implanted region into a simple resistor network made of two parallel resistor arms as shown in figure 7.14 (b).

Model:

$$
\begin{gathered}
R_{t}=r_{t} \cdot T^{m}+c \\
R_{s}=r_{s} \cdot e^{\left(\frac{D}{T}\right)}+C \\
R_{m}=r_{m} T^{n} \\
\frac{1}{R}=\frac{1}{R_{\text {tunnel }}}+\frac{1}{\left(R_{s}+R_{m}\right)}
\end{gathered}
$$




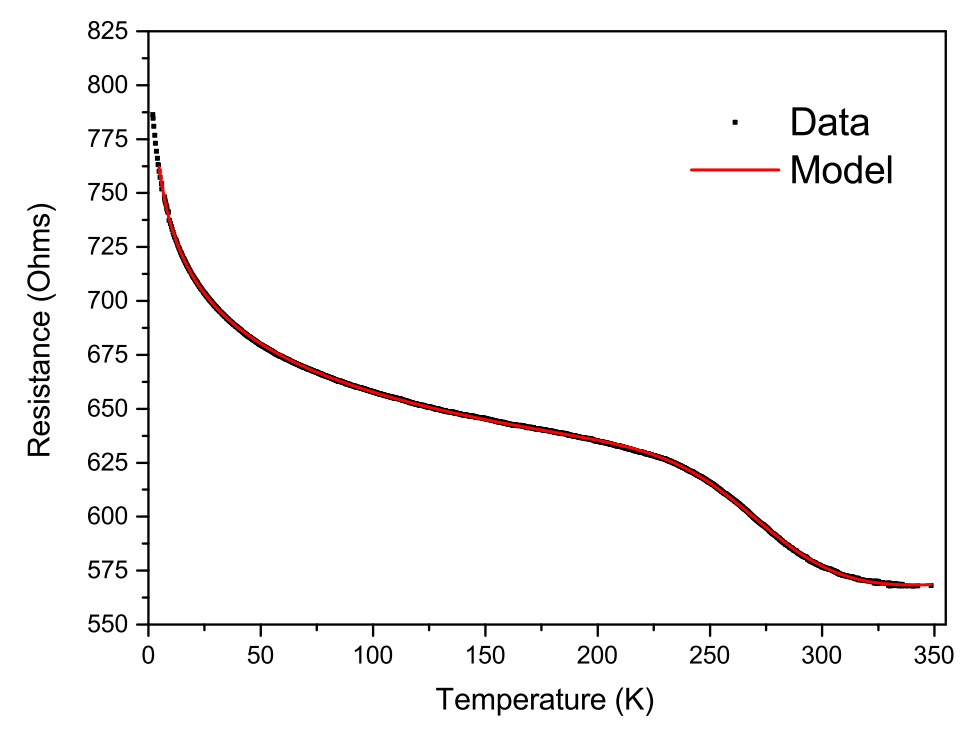

Figure 7.15: The dependence of resistance on measurement temperature is fit with the model for parallel network of conduction displayed in figure 7.14. The fit agrees well with the resistance data.

Figure 7.15 shows the resistance data fit with the above model. The fitting parameters used for the process are given by,

Table 7.1: Fitting parameters for Co 12 transport measurements

\begin{tabular}{|c|c|}
\hline Fitting parameters & Co 12 \\
\hline \hline$r_{t}$ & 820 \\
\hline$m$ & -0.048 \\
\hline$c$ & 1.29 \\
\hline$r_{s}$ & 0.09 \\
\hline$D$ & 3100 \\
\hline$C$ & 0.02 \\
\hline$r_{m}$ & 0.05 \\
\hline$n$ & 2 \\
\hline
\end{tabular}

In the model, $r_{t}, r_{s}$ and $r_{m}$ represent the resistance from tunneling, semiconducting and metallic-like regions. $m$ represents the exponent of the temperature from the tunnel resistance. It can be seen that the temperature dependence is weak which is not surprising considering the inhomogeneous nature of the sample. $D$ is proportional to the activation energy of DLC in the implanted region. D corresponds to an activation energy of $\sim 200$ 
$\mathrm{meV}$. The energy is far from the optical band gap of the material and is hence determined by the extended $\mathrm{sp}^{2}$ states in the DLC layer. The contribution from the metallic-like region can be satisfactorily fit with a temperature exponent of $2(n)$, the low $r_{m}$ denotes the low concentration of metallic-like regions present in the sample.

Sample Co 7 shows similar temperature dependence of resistance. $R$ vs $\log (\mathrm{T})$ plot of the sample is shown below.

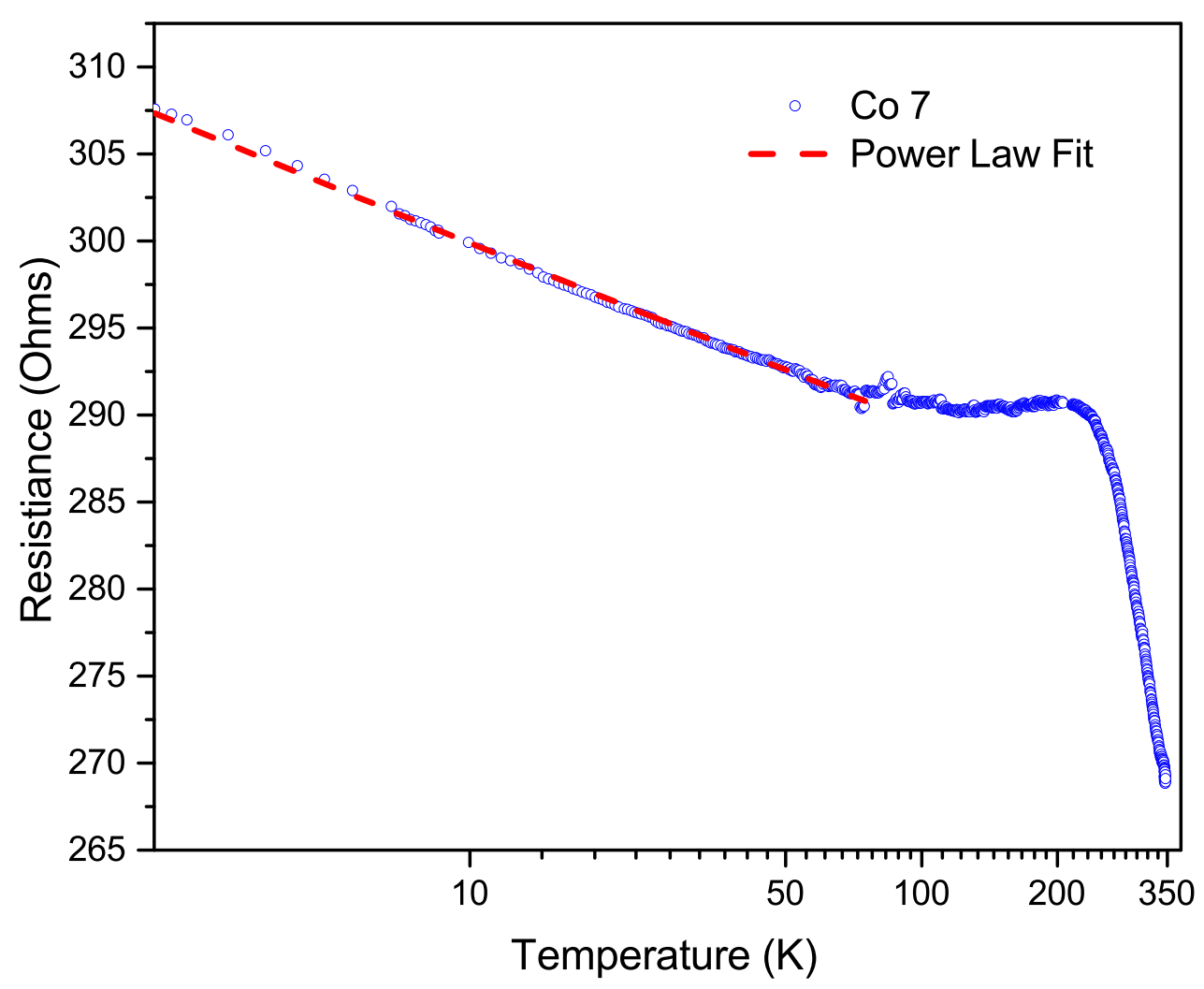

Figure 7.16: Logarithmic temperature dependence of resistance of Co 7 in the low temperature region.

Sample Co 7 also shows a weak power law dependence in the low temperature region as shown in figure 7.16. However a change in implantation fluence causes a change in the nanoparticle distribution and, an additional factor $\left(r_{t 2} T^{m_{2}}\right)$ is required to account for the low temperature dependence of the tunnel resistance. The expression required to fit the data in this case is given by, 


$$
R_{t}=r_{t 1} \cdot T^{m_{1}}+r_{t 2} T^{m_{2}}+c
$$

In the high temperature region, there is a similar deviation which is characteristic of thermal activation behavior. Since the implantation fluence is lower in this case, the spatial distribution of the nanoparticles are not as close as observed in the previous sample. Hence, the contribution from metallic like region observed previously is absent in this sample.

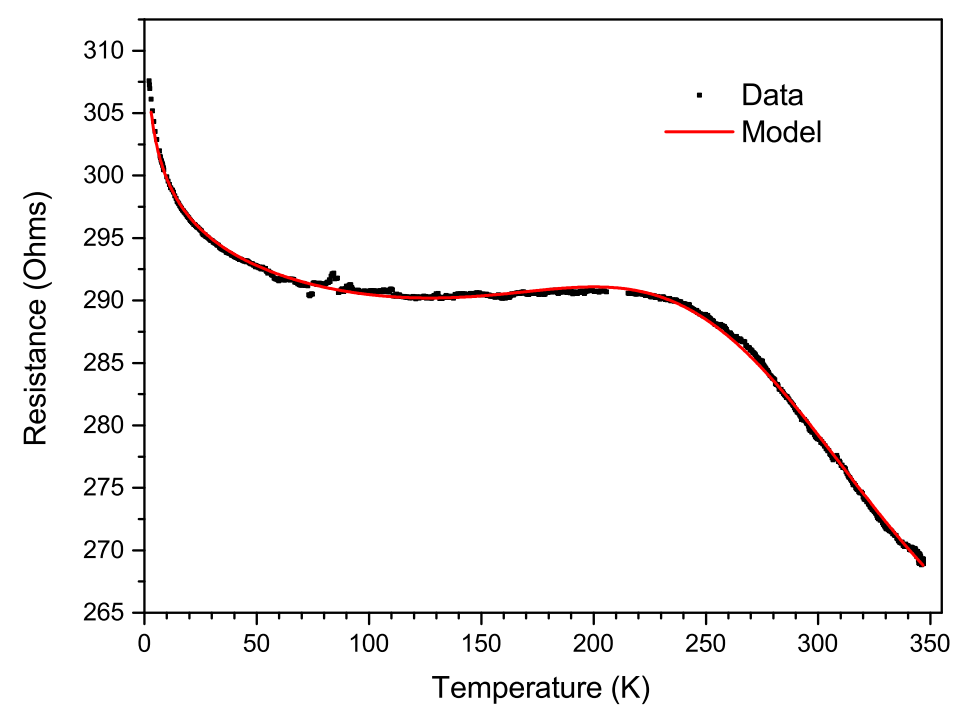

Figure 7.17: Modeled fit of resistance vs temperature data for Co 7.

Figure 7.17 shows the resistance data of sample Co 7 fit by the above explained model. The model can be summarized as below,

$$
\begin{gathered}
R_{t}=r_{t 1} \cdot T^{m_{1}}+r_{t 2} T^{m_{2}}+c \\
R_{s}=r_{s} \cdot e^{\left(\frac{D}{x}\right)}+C \\
\frac{1}{R}=\frac{1}{R_{\text {tunnel }}}+\frac{1}{R_{s}}
\end{gathered}
$$

The fitting parameters used in figure 7.16 are shown in table 7.1. 
Table 7.2: Fitting parameters for Co 7 transport measurements

\begin{tabular}{|c|c|}
\hline Fitting parameters & Co 7 \\
\hline \hline$r_{t 1}$ & 310 \\
\hline$m_{1}$ & -0.015 \\
\hline$r_{t 2}$ & $1.7 \mathrm{E}-6$ \\
\hline$m_{2}$ & 2.83 \\
\hline$c$ & 0.036 \\
\hline$R_{s}$ & 4.49 \\
\hline$D$ & 1933 \\
\hline$C$ & 807 \\
\hline
\end{tabular}

\subsection{Conclusion}

In conclusion, this chapter presents the results from magnetic and transport measurements performed on Co implanted DLC films. Magnetic measurements on Co implanted DLC films showed that the implantation induces a room temperature magnetic order in DLC films. The magnetic order is attributed to the cobalt carbide nanoparticles formed during implantation. The temperature dependency of the saturated moment of the films showed that the nanoparticles are structurally disordered leading to a spin glass behavior. However, increasing the implantation fluence was observed to result in an increase in the ordered ferromagnetic phase of the nanoparticles. A fraction of implanted Co atoms gets diluted within DLC. ZFC-FC measurements indicate the nanoparticles to be superparamagnetic in nature and the dipole-dipole interaction between these nanoparticles were measured to have an enhancing effect on the magnetization of the nanoparticles.

Electrical conduction in Co implanted DLC is a complex mechanism involving parallel conduction pathways through an inhomogeneously distributed array of random nanoparticles. A realistic representation of such system requires 3D modeling of multiple parallel resistor networks where each component can show a unique individual temperature dependency. In this chapter, it is shown that this complex system can be simplified into a resistor network model consisting of two parallel conduction pathways. The major contribution stems from the tunneling between large nanoparticles which is represented in one resistor arm. This resistor shows a weak power law dependence on temperature. At higher temperature an additional contribution was identified which is modeled as a parallel conduction pathway in the resistor network. Depending on the spatial density of the nanoparticles (which is in turn dependent on the implantation fluence) this contribution at higher temperature can be classified into semiconducting contribution and metallic like contribution. Semiconducting contribution arise from the hopping conduction between $\mathrm{sp}^{2}$ clusters with 
an activation energy in the order of $200 \mathrm{meV}$. The metallic-like contribution arise from the scattering effects in closely positioned nanoparticles. In sample Co 12 both semiconducting and metallic-like contribution were identified, while in sample Co 7 the contribution from metallic like regions were found to be negligible. This simplified model satisfactorily fit the resistance data obtained from the Co implanted DLC films. 


\section{Chapter 8}

\section{Summary and Outlook}

\subsection{Summary}

This chapter summarizes the results obtained so far in the research work. The thesis began with the question of "What happens when Co is implanted into DLC?". Specifically, the objective of the research was focused towards measuring the impact of low energy Co implantation on the structure, elemental distribution, magnetic behavior and electron transport of ion beam deposited DLC thin films. The study reveals that ion implantation graphitizes the DLC film, leads to bimodal distribution and nanoparticle formation with a room temperature magnetic order and a transport behavior strongly dominated by the disordered metallic nanoparticles formed during Co implantation.

In detail, DLC films were deposited at room temperature by mass-selective ion beam deposition with $\mathrm{C}_{3} \mathrm{H}_{6}^{+}$ions at $5 \mathrm{keV}$ deposition energy. The films contains about $25 \%$ hydrogen concentration. The $\mathrm{sp}^{3}$ content of the films were measured to be greater than $30 \%$ and its $\mathrm{sp}^{3}$ character was observed to decrease with increasing deposition energy. Similar results were found with direct ion beam depositions.

The as-deposited DLC films were implanted with Co ions at $30 \mathrm{keV}$ energy and an implantation fluence varying from $0.8-20 \times 10^{16}$ atoms.cm ${ }^{-2}$. Even though the implantation was carried out at room temperature, a bimodal distribution of Co ions were measured by HR-RBS for fluence greater than $2.4 \times 10^{16}$ atoms.cm $^{-2}$. The distribution was characterized by formation of a near-surface Co peak followed by a peak at the predicted mean projected range from DTRIM simulations. A region depleted of Co was measured between the surface and center peaks. Increase in implantation fluence resulted in an asymmetric bimodal distribution with greater accumulation of Co in the near surface region. The most prominent bimodal distribution was obtained for a Co dose of $7 \times 10^{16}$ atoms.cm ${ }^{-2}$. The surface peak accumulated more than twice the amount of Co than the center peak. A steady state 
condition was finally achieved at a fluence of $12 \times 10^{16}$ atoms.cm $^{-2}$.

Ion implantation was also observed to cause nanoparticle formation within the implanted films. Cross-sectional TEM imaging revealed the nanoparticles to follow the Co distribution measured by HR-RBS. At high fluences, nanoparticles located at the surface peak was observed to be much larger than the nanoparticles formed at the center peak. For example, at $\mathrm{F}=7 \times 10^{16}$ atoms.cm ${ }^{-2}$, the surface nanoparticles were about $5 \mathrm{~nm}$ in diameter while those nanoparticles formed at the center peak were only $2 \mathrm{~nm}$ in size. The bimodal size distribution and the bimodal Co distribution formed during implantation was explained with a model based on collision cascades, thermal spikes and defect formation.

The model proposed that ion implantation leads to manifestation of two competing processes: nanoparticle formation and localized diffusion. Diffusion leads to broadening of implanted profile reducing the peak concentration, on the other hand nanoparticle formation leads to accumulation of Co leading to a localized increase in Co concentration. Near the predicted range where the collision cascade is expected to have maximum effect, diffusion dominates leading to the depleted region. On the other hands the defects formed during ion implantation acts as traps and nucleating sites for larger nanoparticle growth leading to the surface peak. A crucial factor that makes this model work is the low thermal conductivity of DLC which is mainly attributed to the presence of hydrogen within them. In absence of hydrogen the conductivity rises and similar distribution is not expected. Experimental investigations of Co implantation into amorphous carbon films reveals that bimodal distribution is not observed in absence of hydrogen. This observation verifies the proposed model.

The hydrogen distribution within DLC was measured before and after Co implantation. It was observed that Co implantation leads to massive rearrangement in the hydrogen profile and leads to significant effusion of near-surface hydrogen atoms. The resultant hydrogen profile of the implanted DLC films show again a bimodal distribution. Raman spectroscopy further revealed that the ion implantation leads to graphitization of DLC films. Graphitization implies an increase in the $\mathrm{sp}^{2}$ nature of the films. In the case of hydrogenated DLC, this happens predominantly by increase in the cluster size of $\mathrm{sp}^{2}$ carbon. On the other hand, in the amorphous carbon films, due to absence of hydrogen, the dangling bonds of $\mathrm{sp}^{3}$ carbons generated during ion implantation remains unsatisfied leading to rehybridization of $\mathrm{sp}^{3}$ carbon to $\mathrm{sp}^{2}$ carbon leading to the rise in $\mathrm{sp}^{2}$ character of the DLC films.

Magnetic measurements showed that Co implanted DLC films show room temperature magnetic order. Further the magnetic order was inferred to arise predominantly from the disordered metallic Cobalt carbide nanoparticles formed during implantation. These nanoparticles exhibit a spin glass behavior which shows strong, exponential temperature dependence. It was further found out that increasing the implantation fluence reduces the structural 
disorder which inhibits the spin glass behavior. An increasing structural order leads to manifestation of ordered ferromagnetic phase which can be observed as an additional Bloch contribution to the spin glass magnetic moment in the temperature dependent measurements. ZFC-FC measurements revealed that the nanoparticles have a blocking temperature ranging from $4 \mathrm{~K}$ to $15 \mathrm{~K}$. The particle size was confirmed to increase with implantation fluence. A further interesting effect is the observation of a positive dipole interaction among the nanoparticles leading to a Curie-Weiss behavior observed at the high temperature region of the ZFC-FC measurements. The Weiss temperature was measured to be $71 \mathrm{~K}$ for Co 7 sample. The saturated magnetization further indicated that only $30 \%$ of implanted DLC are present in the nanoparticles. The rest are possibly diluted within the DLC matrix.

Transport measurements showed that in Co implanted DLC, electron transport primarily occur at the Co implanted regions. The dominant conductivity pathway for electron transport was observed to occur through the disordered metallic nanoparticles present at the near-surface region. However at higher temperature a parallel pathway through the small nanoparticles near the predicted range becomes available due to thermal activation. This pathway is mainly semiconducting in nature in the case of Co 7 sample while it also includes a metallic contribution in the case of Co 12 sample. The transport mechanism overall is dominated by the tunneling between the large metallic nanoparticles.

Thus the overall conclusion of the research is that low energy ion implantation into DLC results in manifestation of unique properties seldom reported in literature. These properties could be very useful for understanding the ion-solid interactions in thermal insulators and for interesting magnetic and spintronic applications in the form of spin glass and condensed magnetic semiconductors.

\subsection{Outlook}

Diamond-like carbon since its inception has been predominantly used for their physical and mechanical properties. Even when studies suggested that DLC can be modified to show a wide range of electrical conductivity, DLC did not find applications beyond the coating industry. At the beginning of this work on ion implanted DLC films, there were uncertainties regarding the possible novel aspects of this research. However, Co implanted DLC films showed several features and properties that were not expected or even observed in other materials at the same experimental conditions. This work merely show case some of the many aspects of ion implanted DLC films. The results obtained indicate that it is worth exploring this field in greater detail.

The interest in this field can be split into two separate domains. One domain of interest 
is to explore different combinations of ion species and host materials in the implantation work with an aim to measure similar interactions and effects. For example, ions with mass, heavier and lighter than Co can be experimented to find out their consequent effect on the elemental distribution and DLC structure. Silver or gold is a good choice. They are expected to show useful transport and optical properties. Both these elements are inert and silver especially is reported to show high diffusivity. Structural investigations on Ag or Au implanted DLC can lead to further developments and shed more light on the ion-solid interactions in DLC. Another approach would be to experiment with noble gas ions. Since these ions would not form nanoparticles it would be particularly interesting to study their distribution across DLC. Polymers can be an interesting choice for exploring a variation in the host matrix. Polymers like DLC are composed of light elements and are excellent thermal insulators. The effects due to thermal spikes and collision cascades are thus expected to be even more significant for such materials.

The other domain of interest is to explore other properties of implanted DLC films. This study focused on the structure and magnetic behavior of implanted films. Future work on this research would lead towards exploration of magneto-transport properties of Co implanted DLC films. They can be expected to show quasi linear magnetoresistance and strong temperature dependence which can be useful for sensor applications. Other unexplored area is the optical properties of implanted DLC films. Silver implantation in particular can lead to observation of surface plasmon resonance in DLC. The variation in the electrical and optical band gaps in DLC upon implantation can add interesting perspective to this research.

Another area that is worth exploring is the chemical bonding of the implanted ions with the DLC matrix. Synchrotron X-Ray analysis on the chemical state of $\mathrm{C}$ and Co or any other metallic dopant in the implanted region would shed light on the chemistry involved in this process. Since the interactions occur in short time scale and under extreme conditions, novel and exotic chemical compounds of carbon with the dopant of interest can form during ion implantation.

In short, this work on Co implanted DLC films revealed many interesting aspects which are of significant interest for a wide range of applications. Further explorations in this field could result in novel applications and can reduce the new knowledge gap identified in this research work. 


\section{List of Publications}

\section{Journal Publications}

1. Gupta, P.S.; Williams, G.; Huebner, R.; Vajandar, S.; Osipowicz, T.; Heinig, K.H.; Becker, H.-W.; Markwitz, A. 2017. Self-assembly of magnetic nanoclusters in diamond-like carbon by diffusion processes enhanced by collision cascades. Applied Physics Letters, 110, article no. 141901; doi: 10.1063/1.4979523

2. Gupta, P.S.; Becker, H.-W.; Williams, G.; Huebner, R.; Heinig, K.-H.; Markwitz, A. 2017. Collision cascades enhanced hydrogen redistribution in cobalt implanted hydrogenated diamond-like carbon films. Nuclear Instruments and Methods in Physics Research Section B: Beam Interactions with Materials and Atoms, 394: 6-11; doi: $10.1063 / 1.4979523$

3. Gupta, P.S.; Williams, G.; Markwitz, A. 2016. Ferromagnetic order in diamond-like carbon films by Co implantation. Journal of physics. D, Applied physics, 49(5): article no. 055002; doi: 10.1088/0022-3727/49/5/055002

4. Markwitz, A.; Gupta, P.S.; Mohr, B.; Huebner, R.; Leveneur, J.; Zondervan, A.; Becker, H-W. 2016. Near-surface hydrogen depletion of diamond-like carbon films produced by direct ion deposition. Nuclear instruments \& methods in physics research. Section B, Beam interactions with materials and atoms, 371: 230-234

5. Markwitz, A.; Leveneur, J.; Gupta, P.; Suschke, K.; Futter, R.J.; Rondeau, M. 2015. Transitional metal ion implantation into diamond-like carbon coatings : development of a base material for gas sensing applications. Journal of nanomaterials, 2015: article 706417

6. Suschke, K.; Huebner, R.; Murmu, P.P.; Gupta, P.S.; Futter, R.J.; Markwitz, A. 2015. High energy radial deposition of diamond-like carbon coatings. Coatings, 5: 326-337 


\section{Conference Proceedings}

1. Gupta, P.S.; Williams, G.; Vajandar, S.; Osipowicz, T.; Becker, H.-W.; Heinig, K.-H.; Huebner, R.; Leveneur, J.; Kennedy, J.; Markwitz, A. 2017. Positioning of cobalt atoms in amorphous carbon films by pre-selecting the hydrogen concentration. Nuclear Instruments and Methods in Physics Research Section B: Beam Interactions with Materials and Atoms, In press. https://doi.org/10.1016/j.nimb.2017.04.005

2. Gupta, P.; Williams, G.; Markwitz, A. 2016. Ferromagnetism in a diamond-like carbon film after nickel implantation. International Journal of Nanotechnology, 14, 1-6. doi: 10.1504/IJNT.2017.082449 


\section{Bibliography}

[1] M. Nastasi, J. Mayer, and J. K. Hirvonen, Ion-solid interactions: fundamentals and applications. Cambridge University Press, 1996.

[2] B. D. Cullity and C. D. Graham, Introduction to magnetic materials. John Wiley \& Sons, 2011.

[3] A. Markwitz, B. Mohr, D. F. Carpeno, and R. Hubner, "Ultra-smooth diamond-like carbon coatings with high elasticity deposited at low temperature by direct ion beam deposition," Surface \&5 Coatings Technology, vol. 258, pp. 956-962, 2014. [Online]. Available: $<$ Go to ISI $>$ ://WOS:000346895000116

[4] T. Osipowicz, H. L. Seng, T. K. Chan, and B. Ho, "The ciba high resolution rbs facility," Nuclear Instruments and Methods in Physics Research Section B: Beam Interactions with Materials and Atoms, vol. 249, no. 1â2, pp. 915-917, 2006. [Online]. Available: http://www.sciencedirect.com/science/article/pii/S0168583X06004368

[5] K. U. E. I. CHAN TAW, "High depth resolution rutherford backscattering spectrometry with a magnet spectrometer: Implementation and application to thin film analysis," Ph.D. dissertation, 2009.

[6] J. Bland, "A mossbauer spectroscopy and magnetometry study of magnetic multilayers and oxides," Master's thesis, Oliver Lodge Labs, Dept. Physics, University of Liverpool, 2002.

[7] M. McElfresh, "Fundamentals of magnetism and magnetic measurements featuring quantum designâs magnetic property measurement system," Quantum Design, vol. 11578, p. 132, 1994.

[8] J. Robertson, "Diamond-like amorphous carbon," Materials Science and Engineering: R: Reports, vol. 37, no. 4, pp. 129-281, 2002. 
[9] R. Berman and S. F. Simon, "On the graphiteâdiamond equilibrium," Berichte der Bunsengesellschaft f̃̃AEr physikalische Chemie, vol. 59, no. 5, pp. 333-338, 1955.

[10] A. C. Ferrari and J. Robertson, "Raman spectroscopy of amorphous, nanostructured, diamondâlike carbon, and nanodiamond," Philosophical Transactions of the Royal Society of London A: Mathematical, Physical and Engineering Sciences, vol. 362, no. 1824, pp. 2477-2512, 2004.

[11] L. N. Large and R. W. Bicknell, "Ion-implantation doping of semiconductors," Journal of Materials Science, vol. 2, no. 6, pp. 589-609, Nov 1967. [Online]. Available: https://doi.org/10.1007/BF00752224

[12] J. W. Mayer, J. A. Davies, and L. Eriksson, Ion implantation in semiconductors. Acad. Press New York, 1970.

[13] G. H. Vineyard, "Thermal spikes and activated processes," Radiation Effects, vol. 29, no. 4, pp. 245-248, 2006.

[14] H. Hosono, H. Fukushima, Y. Abe, R. A. Weeks, and R. A. Zuhr, "Cross-sectional tem observation of copper-implanted sio2 glass," Journal of Non-Crystalline Solids, vol. 143, pp. 157-161, 1992. [Online]. Available: http://www.sciencedirect.com/science/article/pii/S0022309305805642

[15] N. Matsunami and H. Hosono, "Colloid formation effects on depth profile of implanted ag in sio2 glass," Applied Physics Letters, vol. 63, no. 15, p. 2050, 1993.

[16] N. Kishimoto, N. Umeda, Y. Takeda, C. G. Lee, and V. T. Gritsyna, "Self-assembled two-dimensional distribution of nanoparticles with high-current cuâ implantation into insulators," Nuclear Instruments and Methods in Physics Research Section B: Beam Interactions with Materials and Atoms, vol. 148, no. 1â4, pp. 1017-1022, 1999. [Online]. Available: http://www.sciencedirect.com/science/article/pii/S0168583X98008763

[17] M. Dubiel, H. Hofmeister, E. Schurig, E. Wendler, and W. Wesch, "On the stress state of silver nanoparticles in ion-implanted silicate glasses," Nuclear Instruments and Methods in Physics Research Section B: Beam Interactions with Materials and Atoms, vol. 166â167, pp. 871-876, 2000. [Online]. Available: http://www.sciencedirect.com/science/article/pii/S0168583X99007971

[18] T. Dietl and H. Ohno, "Dilute ferromagnetic semiconductors: Physics and spintronic structures," Reviews of Modern Physics, vol. 86, no. 1, p. 187, 2014. 
[19] S. A. Wolf, D. D. Awschalom, R. A. Buhrman, J. M. Daughton, S. Von Molnar, M. L. Roukes, A. Y. Chtchelkanova, and D. M. Treger, "Spintronics: a spin-based electronics vision for the future," Science, vol. 294, no. 5546, pp. 1488-1495, 2001.

[20] T. Dietl, "A ten-year perspective on dilute magnetic semiconductors and oxides," Nat Mater, vol. 9, no. 12, pp. 965-974, Dec. 2010. [Online]. Available: http://dx.doi.org/10.1038/nmat2898

[21] Z. Jia, R. Zhang, Q. Han, Q. Yan, R. Zhu, D. Yu, and X. Wu, "Large tunable linear magnetoresistance in gold nanoparticle decorated graphene," Applied Physics Letters, vol. 105, no. 14, p. 143103, 2014. [Online]. Available: http://scitation.aip.org/content/aip/journal/apl/105/14/10.1063/1.4897277

[22] M. A. Ruderman and C. Kittel, "Indirect exchange coupling of nuclear magnetic moments by conduction electrons," Phys. Rev., vol. 96, no. 1, pp. 99-102, Oct. 1954. [Online]. Available: https://link.aps.org/doi/10.1103/PhysRev.96.99

[23] R. K. Kawakami, E. Johnston-Halperin, L. F. Chen, M. Hanson, N. GuÃ@(bels, J. S. Speck, A. C. Gossard, and D. D. Awschalom, "(ga,mn)as as a digital ferromagnetic heterostructure," Applied Physics Letters, vol. 77, no. 15, pp. 2379-2381, 2000. [Online]. Available: http://scitation.aip.org/content/aip/journal/apl/77/15/10.1063/1.1316775

[24] J. Kennedy, G. V. M. Williams, P. P. Murmu, and B. J. Ruck, "Intrinsic magnetic order and inhomogeneous transport in gd-implanted zinc oxide," Physical Review B, vol. 88, no. 21, p. 214423, 2013. [Online]. Available: http://link.aps.org/doi/10.1103/PhysRevB.88.214423

[25] C. B. Fitzgerald, M. Venkatesan, A. P. Douvalis, S. Huber, J. M. D. Coey, and T. Bakas, "Sno 2 doped with mn, fe or co: room temperature dilute magnetic semiconductors," Journal of applied physics, vol. 95, no. 11, pp. 7390-7392, 2004.

[26] A. Numan, N. Alexei, Z. Hartmut, W. Kurt, B. Hans-Werner, S. Christoph, G. Åafak, B. Asif, K. Rustam, and T. Lenar, "High-temperature ferromagnetism in co-implanted tio 2 rutile," Journal of Physics D: Applied Physics, vol. 42, no. 11, p. 115005, 2009. [Online]. Available: http://stacks.iop.org/0022-3727/42/i=11/a=115005

[27] Y. Matsumoto, M. Murakami, T. Shono, T. Hasegawa, T. Fukumura, M. Kawasaki, P. Ahmet, T. Chikyow, S.-y. Koshihara, and H. Koinuma, 
"Room-temperature ferromagnetism in transparent transition metal-doped titanium dioxide," Science, vol. 291, no. 5505, p. 854, 2001. [Online]. Available: http://science.sciencemag.org/content/291/5505/854.abstract

[28] A. Wojcik, K. Kopalko, M. Godlewski, E. Guziewicz, R. JakieÅa, R. Minikayev, and W. Paszkowicz, "Magnetic properties of znmno films grown at low temperature by atomic layer deposition," Applied Physics Letters, vol. 89, no. 5, p. 051907, 2006. [Online]. Available: http://scitation.aip.org/content/aip/journal/apl/89/5/10.1063/1.2245209

[29] J. J. Liu, M. H. Yu, and W. L. Zhou, "Fabrication of mn-doped zno diluted magnetic semiconductor nanostructures by chemical vapor deposition," Journal of Applied Physics, vol. 99, no. 8, p. 08M119, 2006. [Online]. Available: http://scitation.aip.org/content/aip/journal/jap/99/8/10.1063/1.2173235

[30] Y. Fukuma, H. Asada, S. Senba, and T. Koyanagi, "Epitaxial growth of diluted magnetic semiconductor gelâxcrxte with high cr composition," Applied Physics Letters, vol. 108, no. 22, p. 222403, 2016. [Online]. Available: http://scitation.aip.org/content/aip/journal/apl/108/22/10.1063/1.4953042

[31] D. Xu, P. Li, Y. Zhang, Y. Lou, and Y. Li, "Defect formation and its effect on electronic structure and magnetic properties of gan:mn films," Thin Solid Films, vol. 616, pp. 573-578, 2016. [Online]. Available: http://www.sciencedirect.com/science/article/pii/S0040609016305272

[32] J. Leveneur, J. Kennedy, G. Williams, J. Metson, and A. Markwitz, "Large room temperature magnetoresistance in ion beam synthesized surface fe nanoclusters on sio2," Applied Physics Letters, vol. 98, no. 5, pp. 053111-053111-3, 2011.

[33] S. Kazan, F. A. Mikailzade, A. G. Sale, M. Maksutoglu, M. Acikgoz, R. I. Khaibullin, N. I. Khalitov, J. I. Gatiiatova, and V. F. Valeev, "Magnetic properties of co-implanted batio 3 perovskite crystal," Phys. Rev. B, vol. 82, no. 5, p. 054402, Aug. 2010. [Online]. Available: https://link.aps.org/doi/10.1103/PhysRevB.82.054402

[34] J. Kennedy, J. Leveneur, G. Williams, D. Mitchell, and A. Markwitz, "Fabrication of surface magnetic nanoclusters using low energy ion implantation and electron beam annealing," Nanotechnology, vol. 22, p. 115602, 2011.

[35] A. C. Ferrari, "Diamond-like carbon for magnetic storage disks," Surface and coatings technology, vol. 180, pp. 190-206, 2004. 
[36] A. Markwitz, B. Mohr, and J. Leveneur, "Room temperature diamond-like carbon coatings produced by low energy ion implantation," Nuclear Instruments 83 Methods in Physics Research Section B-Beam Interactions with Materials and Atoms, vol. 331, pp. 144-148, 2014. [Online]. Available: < Go to ISI>://WOS:000339144600031

[37] K. Suschke, R. Hubner, P. P. Murmu, P. Gupta, J. Futter, and A. Markwitz, "High energy radial deposition of diamond-like carbon coatings," Coatings, vol. 5, no. 3, pp. 326-337, 2015. [Online]. Available: <Go to ISI >://WOS:000377252600007

[38] A. Markwitz, J. Leveneur, P. Gupta, K. Suschke, J. Futter, and M. Rondeau, "Transition metal ion implantation into diamond-like carbon coatings: Development of a base material for gas sensing applications," Journal of Nanomaterials, 2015. [Online]. Available: <Go to ISI >://WOS:000348803600001

[39] W. Prellier, A. Fouchet, and B. Mercey, "Oxide-diluted magnetic semiconductors: a review of the experimental status," Journal of Physics: Condensed Matter, vol. 15, no. 37, p. R1583, 2003. [Online]. Available: http://stacks.iop.org/0953$8984 / 15 / \mathrm{i}=37 / \mathrm{a}=\mathrm{R} 01$

[40] E. B. Lombardi, "Co in diamond: Ferromagnetic ordering of a transition metal in diamond," Diamond and Related Materials, vol. 17, no. 7, pp. 1345-1348, 2008.

[41] S. R. Shinde, S. B. Ogale, J. S. Higgins, H. Zheng, A. J. Millis, V. N. Kulkarni, R. Ramesh, R. L. Greene, and T. Venkatesan, "Co-occurrence of superparamagnetism and anomalous hall effect in highly reduced cobalt-doped rutile tio $_{2-\delta}$ films," Phys. Rev. Lett., vol. 92, p. 166601, Apr 2004. [Online]. Available: https://link.aps.org/doi/10.1103/PhysRevLett.92.166601

[42] A. J. Silvestre, L. C. J. Pereira, M. R. Nunes, and O. C. Monteiro, "Ferromagnetic order in aged co-doped tio2 anatase nanopowders," Journal of nanoscience and nanotechnology, vol. 12, no. 8, pp. 6850-6854, 2012.

[43] M. Shamsa, W. Liu, A. Balandin, C. Casiraghi, W. Milne, and A. Ferrari, "Thermal conductivity of diamond-like carbon films," Applied physics letters, vol. 89, no. 16, pp. $161921-161921,2006$.

[44] L. Verlet, "Computer" experiments" on classical fluids. i. thermodynamical properties of lennard-jones molecules," Physical review, vol. 159, no. 1, p. 98, 1967.

[45] J. F. Ziegler, J. P. Biersack, and U. Littmark, "The stopping and range of ions in matter, vol. 1," 1, Pergamon Press, New York, 1985. 
[46] P. Sigmund, "Collision theory of displacement damage, ion ranges, and sputtering," Tech. Rep., 1972.

[47] W.-K. Chu, Backscattering spectrometry. Elsevier, 2012.

[48] "Thomas - fermi model," in Semiclassical Theory of Atoms, B.-G. Englert, Ed. Berlin, Heidelberg: Springer Berlin Heidelberg, 1988, pp. 27-129. [Online]. Available: https://doi.org/10.1007/3-540-19204-2 2

[49] J. F. Ziegler, The Stopping and Ranges of Ions in Matter: Handbook of Stopping Cross-Sections for Energetic Ions in All Elements. Elsevier, 2013, vol. 5.

[50] B. S. Yarlagadda, J. E. Robinson, and W. Brandt, "Effective-charge theory and the electronic stopping power of solids," Phys. Rev. B, vol. 17, no. 9, pp. 3473-3483, May 1978. [Online]. Available: https://link.aps.org/doi/10.1103/PhysRevB.17.3473

[51] H. A. Bethe and R. W. Jackiw, "Intermediate quantum mechanics," 1968.

[52] H. B. Ann, "Physik. 5 (1930) 325. f," Bloch Ann. Physik, vol. 16, p. 285, 1933.

[53] E. C. Montenegro, S. A. Cruz, and C. Vargas-Aburto, "A universal equation for the electronic stopping of ions in solids," Physics Letters A, vol. 92, no. 4, pp. 195-202, 1982.

[54] J. C. Eckardt, G. Lantschner, N. R. Arista, and R. A. Baragiola, "Electronic stopping of slow molecular ions in solids," Journal of Physics C: Solid State Physics, vol. 11, no. 21, p. L851, 1978.

[55] D. K. Brice and S. A. Cruz, "Electronic stopping power: Bragg's rule and firsov's theory," Radiation Effects, vol. 43, no. 4-5, pp. 143-148, Jan. 1979. [Online]. Available: http://dx.doi.org/10.1080/01422447908229240

[56] J. Lindhard, M. Scharff, and H. E. Schiott, Range concepts and heavy ion ranges. Munksgaard Copenhagen, 1963.

[57] H. Sugiyama., "Electronic stopping power formula for intermediate energies," Radiation Effects, vol. 56, no. 3-4, pp. 205-211, 1981.

[58] H. Sugiyama.., "Modification of lindhard-scharff-schiott formula for electronic stopping power," Journal of the Physical Society of Japan, vol. 50, no. 3, pp. 929-932, 1981.

[59] H. Sugiyama, "Stopping power formula for intermediate energy electrons," Physics in Medicine and Biology, vol. 30, no. 4, p. 331, 1985. 
[60] W. Moller and W. Eckstein, "Tridyn â a trim simulation code including dynamic composition changes," Nuclear Instruments and Methods in Physics Research Section B: Beam Interactions with Materials and Atoms, vol. 2, no. 1, pp. 814-818, 1984. [Online]. Available: http://www.sciencedirect.com/science/article/pii/0168583X84903215

[61] N. W. Ashcroft and N. D. Mermin, "Solid state physics (holt, rinehart and winston, new york, 1976)," Google Scholar, vol. 403, 2005.

[62] S. William, "Forming semiconductive devices by ionic bombardment," 1957.

[63] S. William., "Semiconductor translating device," $1954 . \quad$ [Online]. Available: https://www.google.com/patents/US2666814

[64] R. S. Ohl, "Semiconductor translating device," 1956.

[65] J. W. Moyer, "Method of making p-nu junction semiconductor unit," 1958.

[66] A. Markwitz and J. Kennedy, "Group-iv and v ion implantation into nanomaterials and elemental analysis on the nanometre scale," International Journal of Nanotechnology, vol. 6, no. 3-4, pp. 369-383, 2009.

[67] E. Heinicke and H. Baumann, "Penning ion source for mp accelerator," Nuclear Instruments and Methods, vol. 74, no. 2, pp. 229-232, 1969.

[68] H. Liebl, Applied charged particle optics. Springer, 2008, vol. 2012.

[69] D. J. W. Mous, R. G. Haitsma, T. Butz, R.-H. Flagmeyer, D. Lehmann, and J. Vogt, "The novel ultrastable hvee $3.5 \mathrm{mv}$ singletronât accelerator for nanoprobe applications," Nuclear Microprobe Technology and Applications, vol. 130, no. 1, pp. 31-36, Jul. 1997. [Online]. Available: http://www.sciencedirect.com/science/article/pii/S0168583X97001869

[70] M. Mayer, "Simnra, a simulation program for the analysis of nra, rbs and erda," AIP Conference Proceedings, vol. 475, no. 1, pp. 541-544, 1999. [Online]. Available: http://scitation.aip.org/content/aip/proceeding/aipcp/10.1063/1.59188

[71] M. Mayer., "Improved physics in simnra 7," Nuclear Instruments and Methods in Physics Research Section B: Beam Interactions with Materials and Atoms, vol. 332, pp. 176-180, 2014. [Online]. Available: http://www.sciencedirect.com/science/article/pii/S0168583X14003139

[72] D. B. Williams and C. B. Carter, "The transmission electron microscope," in Transmission electron microscopy. Springer, 1996, pp. 3-17. 
[73] A. Thust, J. Barthel, and K. Tillmann, "Fei titan 80-300 tem," Journal of large-scale research facilities JLSRF, vol. 2, p. 41, 2016.

[74] H.-W. Becker and D. Rogalla, "Nuclear reaction analysis," in Neutron Scattering and Other Nuclear Techniques for Hydrogen in Materials. Springer, 2016, pp. 315-336.

[75] F. Traeger, M. Kauer, C. Woll, D. Rogalla, and H.-W. Becker, "Analysis of surface, subsurface, and bulk hydrogen in zno using nuclear reaction analysis," Physical Review B, vol. 84, no. 7 , p. $075462,2011$.

[76] D. Endisch, H. Sturm, and F. Rauch, "Nuclear reaction analysis of hydrogen at levels below 10 at. ppm," Nuclear Instruments and Methods in Physics Research Section B: Beam Interactions with Materials and Atoms, vol. 84, no. 3, pp. 380-392, 1994.

[77] M. Wilde and K. Fukutani, "Hydrogen detection near surfaces and shallow interfaces with resonant nuclear reaction analysis," Surface Science Reports, vol. 69, no. 4, pp. 196-295, 2014.

[78] W. A. Lanford, "Analysis for hydrogen by nuclear reaction and energy recoil detection," Nuclear Instruments and Methods in Physics Research Section B: Beam Interactions with Materials and Atoms, vol. 66, no. 1-2, pp. 65-82, 1992.

[79] C. V. Raman, "A new radiation," 1928.

[80] C. Donnet and A. Erdemir, Tribology of diamond-like carbon films: fundamentals and applications. Springer Science \& Business Media, 2007.

[81] E. G. Spencer, P. H. Schmidt, D. C. Joy, and F. J. Sansalone, "Ionâbeamâdeposited polycrystalline diamondlike films," Applied physics letters, vol. 29, no. 2, pp. 118-120, 1976.

[82] Y. Lifshitz, G. D. Lempert, E. Grossman, I. Avigal, C. Uzan-Saguy, R. Kalish, J. Kulik, D. Marton, and J. W. Rabalais, "Growth mechanisms of dlc films from c + ions: experimental studies," Diamond and Related Materials, vol. 4, no. 4, pp. 318-323, 1995.

[83] Y. Lifshitz, "Hydrogen-free amorphous carbon films: correlation between growth conditions and properties," International Journal on the Science and Technology of Diamond and Related Materials, vol. 5, no. 3, pp. 388-400, Apr. 1996. [Online]. Available: http://www.sciencedirect.com/science/article/pii/0925963595004459

[84] D. R. McKenzie, D. Muller, and B. A. Pailthorpe, "Compressive-stress-induced formation of thin-film tetrahedral amorphous carbon," Physical Review Letters, vol. 67, no. 6, p. 773, 1991. 
[85] D. R. McKenzie, "Generation and applications of compressive stress induced by low energy ion beam bombardment," Journal of Vacuum Science 85 Technology B: Microelectronics and Nanometer Structures Processing, Measurement, and Phenomena, vol. 11, no. 5, pp. 1928$1935,1993$.

[86] J. Robertson, "Deposition mechanisms for promoting sp3 bonding in diamond-like carbon," Diamond and related materials, vol. 2, no. 5-7, pp. 984-989, 1993.

[87] J. Robertson., "The deposition mechanism of diamond-like ac and ac: H," Diamond and Related Materials, vol. 3, no. 4-6, pp. 361-368, 1994.

[88] J.-P. Hirvonen, J. Koskinen, M. Kaukonen, R. Nieminen, and H.-J. Scheibe, "Dynamic relaxation of the elastic properties of hard carbon films," Journal of applied physics, vol. 81, no. 11, pp. 7248-7254, 1997.

[89] H. Hofsass, H. Feldermann, R. Merk, M. Sebastian, and C. Ronning, "Cylindrical spike model for the formation of diamondlike thin films by ion deposition," Applied Physics A, vol. 66, no. 2 , pp. 153-181, 1998.

[90] P. Gupta, H. W. Becker, G. V. M. Williams, R. Heubner, K. H. Heinig, and A. Markwitz, "Collision cascades enhanced hydrogen redistribution in cobalt implanted hydrogenated diamond-like carbon films," Nuclear Instruments and Methods in Physics Research Section B: Beam Interactions with Materials and Atoms, vol. 394, pp. 6-11, 2017. [Online]. Available: http://www.sciencedirect.com/science/article/pii/S0168583X1630564X

[91] A. Markwitz, P. Gupta, B. Mohr, R. Hubner, J. Leveneur, A. Zondervan, and H. W. Becker, "Near-surface hydrogen depletion of diamond-like carbon films produced by direct ion deposition," Nuclear Instruments 8 Methods in Physics Research Section B-Beam Interactions with Materials and Atoms, vol. 371, pp. 230-234, 2016. [Online]. Available: $<$ Go to ISI $>$ ://WOS:000373412000044

[92] A. C. Ferrari, "Determination of bonding in diamond-like carbon by raman spectroscopy," Diamond and Related Materials, vol. 11, no. 3, pp. 1053-1061, 2002.

[93] P. Gupta, G. Williams, and A. Markwitz, "Ferromagnetic order in diamond-like carbon films by co implantation," Journal of Physics D-Applied Physics, vol. 49, no. 5, p. 055002, 2016. [Online]. Available: < Go to ISI $>$ ://WOS:000368944100006

[94] A. L. Stepanov, D. E. Hole, and P. D. Townsend, "Formation of silver nanoparticles in sodaâlime silicate glass by ion implantation near room temperature," Journal 
of Non-Crystalline Solids, vol. 260, no. 1â2, pp. 65-74, 1999. [Online]. Available: http://www.sciencedirect.com/science/article/pii/S002230939900561X

[95] H. Hideo, "Simple criterion on colloid formation in sio 2 glasses by ion implantation," Japanese Journal of Applied Physics, vol. 32, no. 9R, p. 3892, 1993. [Online]. Available: http://stacks.iop.org/1347-4065/32/i=9R/a $=3892$

[96] A. Paul, T. Laurila, V. Vuorinen, and S. V. Divinski, Fickâs Laws of Diffusion. Cham: Springer International Publishing, 2014, pp. 115-139. [Online]. Available: http://dx.doi.org/10.1007/978-3-319-07461-0 3

[97] T. Prakash, G. V. M. Williams, J. Kennedy, and S. Rubanov, "Formation of magnetic nanoparticles by low energy dual implantation of ni and fe into sio2," Journal of Alloys and Compounds, vol. 667, pp. 255-261, 2016. [Online]. Available: http://www.sciencedirect.com/science/article/pii/S0925838816301736

[98] F. R. Brotzen, P. J. Loos, and D. P. Brady, "Thermal conductivity of thin sio2 films," Thin Solid Films, vol. 207, no. 1-2, pp. 197-201, 1992.

[99] A. Barna, B. Pecz, and M. Menyhard, "Tem sample preparation by ion milling/amorphization," Micron, vol. 30, no. 3, pp. 267-276, 1999.

[100] J. A. C. Santana, R. Skomski, V. Singh, V. Palshin, A. Petukhov, Y. B. Losovyj, A. Sokolov, P. A. Dowben, and I. Ketsman, "Magnetism of cr-doped diamond-like carbon," Journal of Applied Physics, vol. 105, no. 7, p. 07A930, 2009. [Online]. Available: http://scitation.aip.org/content/aip/journal/jap/105/7/10.1063/1.3072828

[101] R. Paul, M. K. Sharma, R. Chatterjee, S. Hussain, R. Bhar, and A. K. Pal, "Ferromagnetism in nanocrystalline nickel incorporated diamond-like carbon thin films," Applied Surface Science, vol. 258, no. 15, pp. 5850-5857, 2012. [Online]. Available: http://www.sciencedirect.com/science/article/pii/S0169433212003595

[102] Z. W. Fan, P. Li, E. Y. Jiang, and H. L. Bai, "Evolution of magnetoresistance mechanisms in granular co/c films with different conduction regimes," Journal of Physics D: Applied Physics, vol. 46, no. 6, p. 065002, 2013. [Online]. Available: http://stacks.iop.org/0022-3727/46/i=6/a=065002

[103] Y. Zhang, G. S. Chaubey, C. Rong, Y. Ding, N. Poudyal, P.-c. Tsai, Q. Zhang, and J. P. Liu, "Controlled synthesis and magnetic properties of hard magnetic co x c $(x=2,3)$ nanocrystals," Journal of Magnetism and Magnetic Materials, vol. 323, no. 11, pp. 1495-1500, 2011. 
[104] A. A. El-Gendy, M. Qian, Z. J. Huba, S. N. Khanna, and E. E. Carpenter, "Enhanced magnetic anisotropy in cobalt-carbide nanoparticles," Applied Physics Letters, vol. 104, no. 2, p. $023111,2014$.

[105] K. J. Carroll, Z. J. Huba, S. R. Spurgeon, M. Qian, S. N. Khanna, D. M. Hudgins, M. L. Taheri, and E. E. Carpenter, "Magnetic properties of co2c and co3c nanoparticles and their assemblies," Applied Physics Letters, vol. 101, no. 1, p. 012409, 2012.

[106] P. V. Hendriksen, S. Linderoth, and P.-A. Lindgard, "Finite-size modifications of the magnetic properties of clusters," Physical Review B, vol. 48, no. 10, p. 7259, 1993.

[107] R. Aquino, J. Depeyrot, M. H. Sousa, F. A. Tourinho, E. Dubois, and R. Perzynski, "Magnetization temperature dependence and freezing of surface spins in magnetic fluids based on ferrite nanoparticles," Physical Review B, vol. 72, no. 18, p. 184435, 2005.

[108] P. Crespo, J. M. Gonzalez, A. Hernando, and F. J. Yndurain, "Spin-wave excitations in ribbon-shaped fe nanoparticles," Physical Review B, vol. 69, no. 1, p. 012403, 2004.

[109] K. Binder and A. P. Young, "Spin glasses: Experimental facts, theoretical concepts, and open questions," Reviews of Modern physics, vol. 58, no. 4, p. 801, 1986.

[110] C. P. Bean and u. D. Livingston, "Superparamagnetism," Journal of Applied Physics, vol. 30, no. 4, pp. S120-S129, 1959.

[111] E. Carpenter, A. A. El-Gendy, and S. Khanna, "High anisotropy nanoparticles," 2016. [Online]. Available: https://www.google.co.nz/patents/US20160159653

[112] J. Osuna, D. de Caro, C. Amiens, B. Chaudret, E. Snoeck, M. Respaud, J.-M. Broto, and A. Fert, "Synthesis, characterization, and magnetic properties of cobalt nanoparticles from an organometallic precursor," The Journal of Physical Chemistry, vol. 100, no. 35, pp. 14571-14 574, 1996. 